Old Dominion University

ODU Digital Commons

Physics Theses \& Dissertations

Physics

Summer 2017

\title{
Nuclear Chiral Axial Currents and Applications to Few-Nucleon Systems
}

Alessandro Baroni

Old Dominion University

Follow this and additional works at: https://digitalcommons.odu.edu/physics_etds

Part of the Nuclear Commons

\section{Recommended Citation}

Baroni, Alessandro. "Nuclear Chiral Axial Currents and Applications to Few-Nucleon Systems" (2017). Doctor of Philosophy (PhD), Dissertation, Physics, Old Dominion University, DOI: 10.25777/rq4d-qq26 https://digitalcommons.odu.edu/physics_etds/11

This Dissertation is brought to you for free and open access by the Physics at ODU Digital Commons. It has been accepted for inclusion in Physics Theses \& Dissertations by an authorized administrator of ODU Digital Commons. For more information, please contact digitalcommons@odu.edu. 


\title{
NUCLEAR CHIRAL AXIAL CURRENTS AND
} APPLICATIONS TO FEW-NUCLEON SYSTEMS

by

\author{
Alessandro Baroni \\ B.Sc. January 2007, University of Pisa, Italy \\ M.Sc. February 2011, University of Pisa, Italy \\ A Dissertation Submitted to the Faculty of \\ Old Dominion University in Partial Fulfillment of the \\ Requirements for the Degree of \\ DOCTOR OF PHILOSOPHY \\ PHYSICS \\ OLD DOMINION UNIVERSITY \\ August 2017
}

Approved by:

Rocco Schiavilla (Director)

Jennifer Georgen (Member)

Alexander Gurevich (Member)

Anatoly Radyushkin (Member)

Lawrence Weinstein (Member) 


\title{
ABSTRACT \\ NUCLEAR CHIRAL AXIAL CURRENTS AND APPLICATIONS TO FEW-NUCLEON SYSTEMS
}

\author{
Alessandro Baroni \\ Old Dominion University, 2017 \\ Director: Dr. Rocco Schiavilla
}

This Thesis is divided into three main parts. The first part discusses basic aspects of chiral effective field theory and the formalism, based on time ordered perturbation theory, used to to derive the nuclear potentials and currents from the chiral Lagrangians. The second part deals with the actual derivation, up to one loop, of the two-nucleon potential and one- and two-nucleon weak axial charge and current. In both derivations ultraviolet divergences generated by loop corrections are isolated using dimensional regularization. The resulting axial current is finite and conserved in the chiral limit, while the axial charge requires renormalization. A complete set of contact terms for the axial charge up to the relevant order in the power counting is constructed. The third part of this Thesis discusses two applications: (i) the calculation of the Gamow-Teller matrix element of tritium, used to constrain the single low-energy constant entering the axial current; (ii) the calculation of neutrino-deuteron inclusive cross sections at low energies. These results have confirmed previous predictions obtained in phenomenological approaches. These latter studies have played an important role in the analysis and interpretation of experiments at the Sudbury Neutrino Observatory. 
Copyright, 2017, by Alessandro Baroni, All Rights Reserved. 


\section{ACKNOWLEDGEMENTS}

Here, I would like to thank the many people who have helped me during these years of graduate studies. First, I would like to express my gratitude to my advisor Prof. Rocco Schiavilla. I would like to thank him for his encouragement, guidance and for providing me interesting projects to work on. In particular I would like to thank him for teaching me the importance of understanding the physical meaning of a theory using simple arguments instead of fancy or shiny formalism.

I am grateful to all of the faculty and staff of the Old Dominion University Physics Department for promoting a stimulating academic and social environment. I would also like to thank all the members of the Theory Group at Jefferson Lab for their hospitality. The interaction with them and the attendance of the numerous organized seminars helped my scientific growth. Other people I have worked with that I would like to thank are Luca Girlanda, Saori Pastore, Maria Piarulli, and Michele Viviani. They provided me with fun discussions. In particular, I would like to thank Maria Piarulli and Jennifer Rautiola for helping me in the early years of my staying in Norfolk, and for their successful efforts to make me feel at home.

A special thanks goes to the vast group of friends I built in Hampton Roads over the years, including Paolo Pezzulo, Gianluca De Leo, Paolo Caricasole, Bruno Zerbo, Maurizio Geri, Pietro Calacione, Fotis Drakopoulos and many others whom I do not have space to mention but for whom I have no less gratitude. Finally, but most important, I would like to thank my family - my parents, Paolo and Susj, my sisters, Elisabetta and Jen - and my

girlfriend Karla for the moral (and not only..) support over the years. In particular I would like to thank Karla for her patience and care for me. 


\section{TABLE OF CONTENTS}

Page

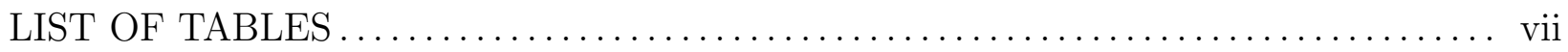

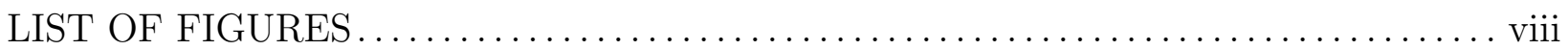

Chapter

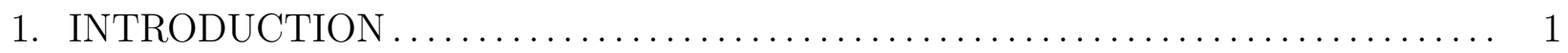

2. CHIRAL EFFECTIVE FIELD THEORY $\ldots \ldots \ldots \ldots \ldots \ldots \ldots \ldots \ldots \ldots \ldots \ldots \ldots . . \ldots$

2.1 CHIRAL SYMMETRY AND THE QCD LAGRANGIAN . . . . . . . . . . 4

2.2 EFFECTIVE LAGRANGIAN FOR PIONS $\ldots \ldots \ldots \ldots \ldots \ldots \ldots \ldots \ldots$

2.3 EFFECTIVE LAGRANGIANS FOR NUCLEONS AND PIONS . . . . . . . 12

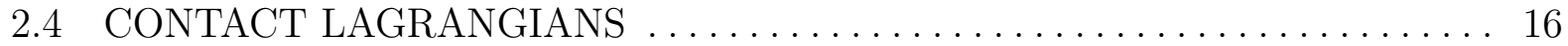

3. NUCLEAR CHIRAL EFFECTIVE FIELD THEORY $\ldots \ldots \ldots \ldots \ldots \ldots \ldots \ldots \ldots \ldots .17$

3.1 INTERACTION HAMILTONIANS FROM CHIRAL LAGRANGIANS . . . . 17

3.2 NUCLEAR POTENTIALS FROM FIELD THEORY AMPLITUDES . . . . . 19

3.3 NUCLEAR CURRENTS FROM FIELD THEORY AMPLITUDES $\ldots \ldots \ldots .22$

4. CHIRAL POTENTIAL AT ONE LOOP $\ldots \ldots \ldots \ldots \ldots \ldots \ldots \ldots \ldots \ldots \ldots \ldots \ldots \ldots . \ldots \ldots$

4.1 STATIC POTENTIAL UP TO ORDER $Q^{2} \ldots \ldots \ldots \ldots \ldots \ldots \ldots \ldots \ldots \ldots . \ldots \ldots$

4.2 NONSTATIC CORRECTIONS TO THE POTENTIAL $\ldots \ldots \ldots \ldots \ldots \ldots \ldots$

5. NUCLEAR AXIAL CURRENTS UP TO ONE LOOP $\ldots \ldots \ldots \ldots \ldots \ldots \ldots \ldots \ldots \ldots$

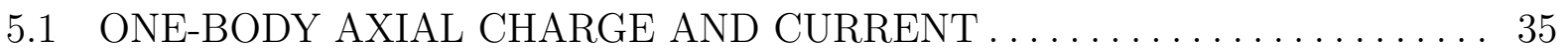

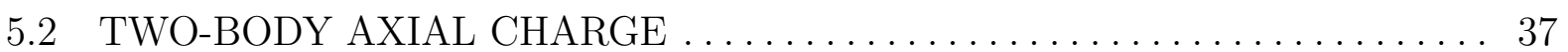

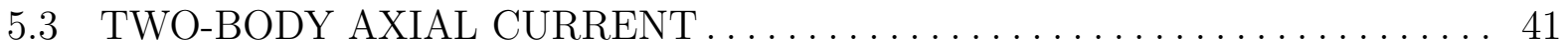

5.4 LOOP CORRECTIONS TO ONE-PION EXCHANGE . . . . . . . . . . 47

5.5 SUMMARY OF AXIAL CHARGE AND CURRENT UP TO ONE LOOP . . 55

5.6 CURRENT CONSERVATION IN THE CHIRAL LIMIT . . . . . . . . . . 58

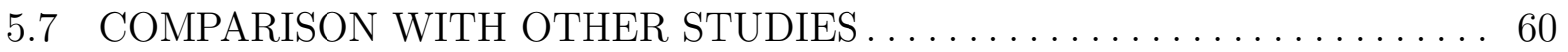

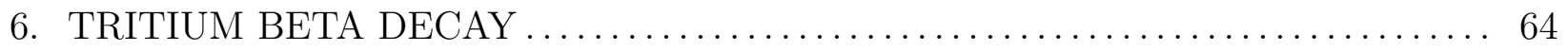

6.1 CONNECTION BETWEEN THE THREE-NUCLEON FORCE AND THE

CONTACT AXIAL CURRENT . . . . . . . . . . . . . . . . . . 64

6.2 NUCLEAR WEAK CURRENTS $\ldots \ldots \ldots \ldots \ldots \ldots \ldots \ldots \ldots \ldots \ldots \ldots$

6.3 GAMOW-TELLER MATRIX ELEMENT IN TRITIUM $\beta$-DECAY $\ldots . . . .68$ 
7. INCLUSIVE NEUTRINO SCATTERING OFF THE DEUTERON AT LOW ENER-

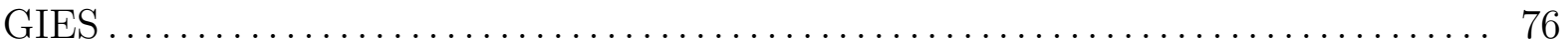

7.1 NEUTRINO INCLUSIVE CROSS SECTION $\ldots \ldots \ldots \ldots \ldots \ldots \ldots \ldots \ldots \ldots$

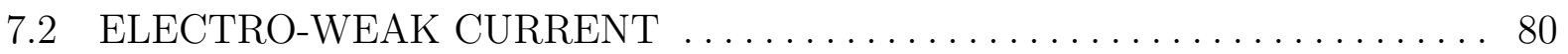

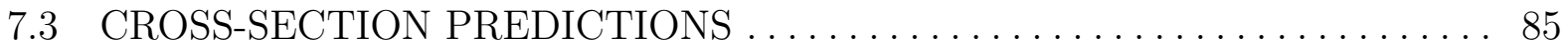

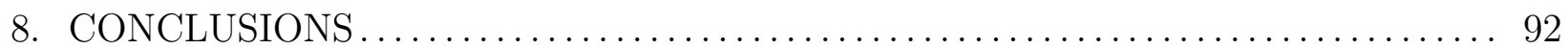

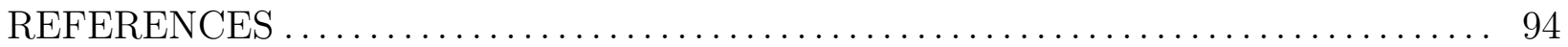

APPENDICES

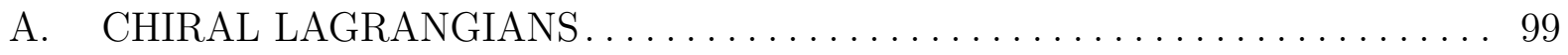

A.1 $\pi \pi$ SECTOR . . . . . . . . . . . . . . . . . . . . . . . . . . . . . . 100

A.2 $\pi N$ SECTOR .......................................... 101

B. INTERACTION VERTICES . . . . . . . . . . . . . . . . . . . 107

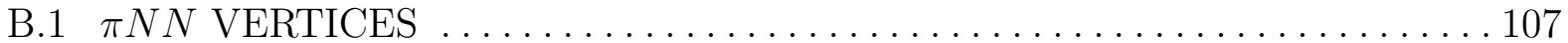

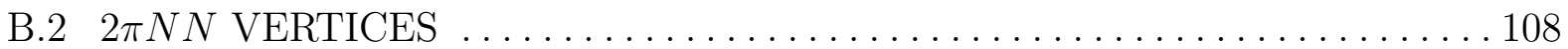

B.3 $3 \pi N N$ VERTEX ......................................... 109

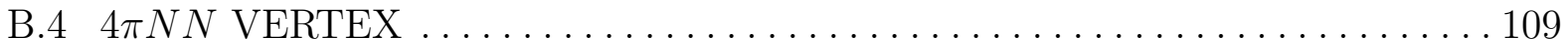

B.5 NNA VERTICES . . . . . . . . . . . . . . . . . . . . . . . . . . . . 110

B.6 $\pi N N A$ VERTICES . . . . . . . . . . . . . . . . . . . . . . . . . . . . . . . . 110

B.7 $2 \pi N N A$ VERTICES . . . . . . . . . . . . . . . . . . . . . . . . . . . . . . 111

B.8 $3 \pi N N A$ VERTICES . . . . . . . . . . . . . . . . . . . . . . . . . . . . 111

B.9 $2 \pi$ VERTICES . . . . . . . . . . . . . . . . . . . . . . . . . . . . . . 112

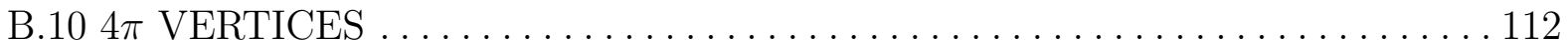

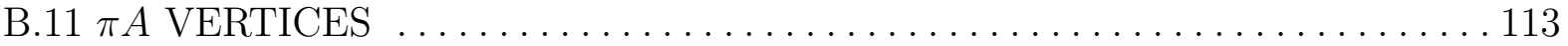

B.12 $3 \pi A$ VERTEX ......................................... 113

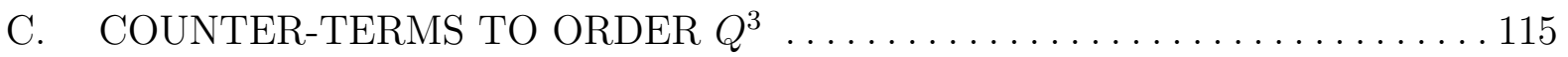

D. FIELD AND MASS RENORMALIZATION OF THE NUCLEON AND PION 117

E. RENORMALIZATION OF THE RATIO $g_{A} / f_{\pi} \ldots \ldots \ldots \ldots \ldots \ldots \ldots \ldots 121$

F. RENORMALIZATION OF THE ONE-BODY AXIAL CURRENT $\ldots \ldots \ldots \ldots 123$

G. CONTACT TERMS FOR THE AXIAL CHARGE CURRENT . . . . . . . . . 126

H. NONSTATIC CORRECTIONS TO ONE-BODY PION-POLE DIAGRAM . . . 128

I. REGULARIZED LOOP CONTRIBUTIONS TO $\mathrm{j}_{5, a}^{\mathrm{MPE}} \ldots \ldots \ldots \ldots \ldots \ldots \ldots$

J. LEADING ORDER AXIAL CHARGE IN COVARIANT PERTURBATION THEORY . . . . . ................................... 132

K. CONFIGURATION-SPACE EXPRESSIONS . .................... 134

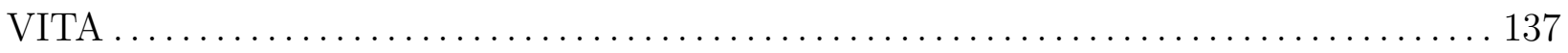




\section{LIST OF TABLES}

Table

1. Contributions to the GT matrix element of tritium $\beta$-decay corresponding to the Hamiltonian model N3LO/N2LO (AV18/UIX) and cutoffs $\Lambda=500 \mathrm{MeV}$ and 600 $\mathrm{MeV}$ in the chiral potentials and weak axial current operators. . . . . . . . 70

2. Adimensional values of the LECs corresponding to the AV18/UIX Hamiltonian model and cutoffs $\Lambda=500 \mathrm{MeV}$ and $600 \mathrm{MeV}$ in the chiral axial current. . . . . 71

3. Values for the $\left(c_{D}, c_{E}\right)$ LECs as obtained by fitting the $A=3$ binding energy and $\mathrm{GT}_{\text {EXP }}$ (its central value), using the N3LO/N2LO potential models with cutoffs $\Lambda=500 \mathrm{MeV}$ and $600 \mathrm{MeV} \ldots \ldots \ldots \ldots \ldots \ldots \ldots \ldots \ldots \ldots \ldots \ldots \ldots \ldots$

4. The LECs in units of powers of $1 / \Lambda$ ( $\Lambda$ is the short-range cutoff $)$ as in Eq. (317). 84

5. Total cross sections in $\mathrm{fm}^{2}$, corresponding to cutoff $\Lambda=500 \mathrm{MeV}$, for the CCinduced processes on the deuteron at selected initial neutrino energies and at

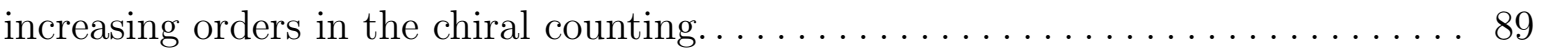

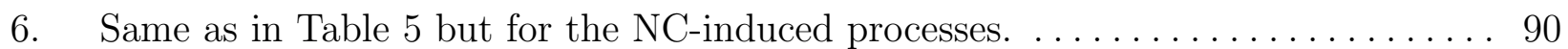




\section{LIST OF FIGURES}

Figure

1. Diagrams illustrating the contribution to the $N N$ potential entering at $\mathrm{LO} Q^{0}$, panels a0 and a1, and $\mathrm{N}^{2} \mathrm{LO}\left(Q^{2}\right)$, panels a2-a8.

2. Half of the possible time-ordered corrections to the OPE potential at order $Q \ldots$. . 30

3. Some of the diagrams illustrating the nonstatic corrections to diagram a3 and a5 of Fig. (1) at order $Q^{3}$

4. Diagrams a1 and a2 contribute to the one-body axial current operator at order $Q^{(-3)}$

5. Diagrams illustrating the relativistic corrections to the one-body axial current. . . 37

6. Diagrams contributing to the OPE axial charge at leading order $Q^{-1}$ (panels b1

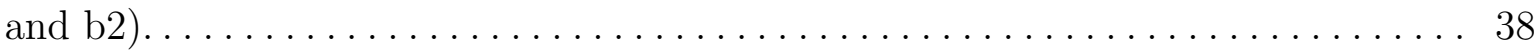

7. Diagrams contributing to the OPE axial charge at order $Q^{0} \ldots \ldots \ldots \ldots$

8. Diagrams contributing to the OPE axial charge at leading order $Q^{-1}$ (panels a1 and a2), and to the TPE axial charge operator at order $Q \ldots \ldots \ldots \ldots \ldots \ldots$

9. Contact and corresponding pion-pole term occuring at order $Q^{0}$ in the axial current. 42

10. Diagrams contributing to the OPE axial current operator at order $Q^{0}$ and to the

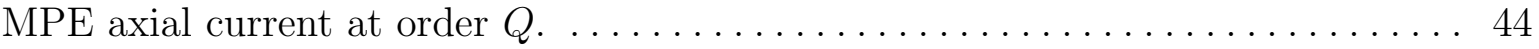

11. Half of the possible time-ordered non-pole corrections to the OPE axial charge

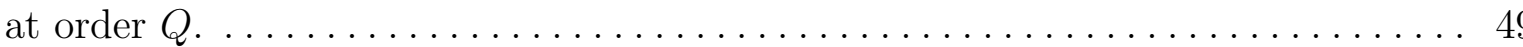

12. Representative diagrams for each of the relevant classes contributing to pole corrections to the OPE axial charge at order $Q \ldots \ldots \ldots \ldots \ldots \ldots \ldots \ldots \ldots \ldots \ldots \ldots \ldots$

13. Additional loop and tree-level corrections of order $Q$ to the OPE axial charge. . . 52

14. The only non-vanishing loop corrections to the OPE axial current. ....... 55

15. Diagrams illustrating the three-body axial current at N4LO (i.e., order $Q^{-2}$ in a

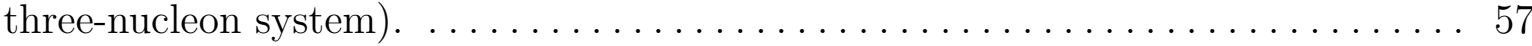

16. Illustration of some of the non-static corrections to the axial current reported in Appendix H. 
17. Diagrams contributing to the axial charge (n1-n2) and current (n3) at order $Q$

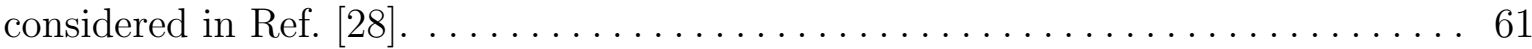

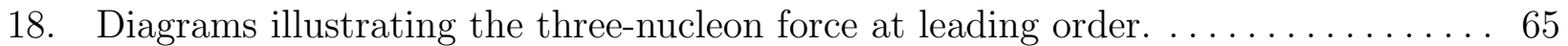

19. The ratio $\mathrm{GT}_{\mathrm{TH}} / \mathrm{GT}_{\mathrm{EXP}}$ as function of the LEC $c_{D}$ obtained retaining corrections

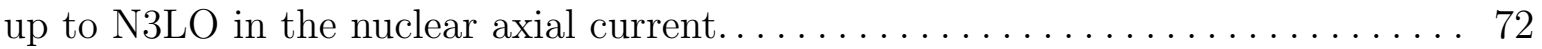

20. Same as Fig. 19 but with the corrections in the axial current up to N4LO. . . . . 72

21. The $c_{D}-c_{E}$ trajectories fitted to reproduce the experimental $A=3$ binding energies and the doublet $n d$ scattering length using the N3LO/N2LO potential with

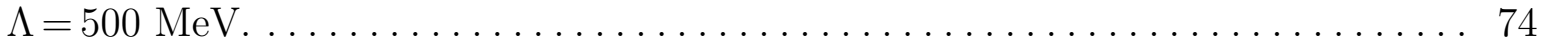

22. Same as Fig. 21 but for $\Lambda=600 \mathrm{MeV} \ldots \ldots \ldots \ldots \ldots \ldots \ldots \ldots \ldots \ldots \ldots \ldots$

23. Diagrams illustrating one- and two-body electromagnetic currents entering at $Q^{-2}$ $(\mathrm{LO}), Q^{-1}(\mathrm{NLO}), Q^{0}(\mathrm{~N} 2 \mathrm{LO})$, and $Q^{1}(\mathrm{~N} 3 \mathrm{LO}) \ldots \ldots \ldots \ldots \ldots \ldots \ldots \ldots \ldots \ldots \ldots \ldots \ldots$

24. Diagrams illustrating one- and two-body electromagnetic charge operators entering at $Q^{-3}(\mathrm{LO}), Q^{-1}(\mathrm{~N} 2 \mathrm{LO}), Q^{0}(\mathrm{~N} 3 \mathrm{LO}), Q^{1}(\mathrm{~N} 4 \mathrm{LO}) \ldots \ldots \ldots \ldots \ldots \ldots$

25. Diagrams illustrating one- and two-body axial currents entering at $Q^{-3}$ (LO), $Q^{-1}(\mathrm{~N} 2 \mathrm{LO}), Q^{0}(\mathrm{~N} 3 \mathrm{LO})$, and $Q^{1}(\mathrm{~N} 4 \mathrm{LO}) \ldots \ldots \ldots \ldots \ldots \ldots \ldots \ldots \ldots \ldots \ldots \ldots \ldots \ldots \ldots$

26. Diagrams illustrating one- and two-body axial charge operators entering at $Q^{-2}$

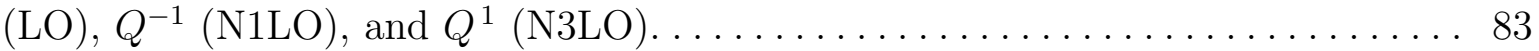

27. Total cross sections in $\mathrm{fm}^{2}$ for the $\nu_{e}$-CC induced process on the deuteron. . . . 86

28. (Color online) Same as in Fig. 27 but for the $\bar{\nu}_{e}-\mathrm{CC}$ induced process on the deuteron. 87

29. Same as in Fig. 27 but for the $\nu_{e}$-NC induced process on the deuteron. . . . . . . 88

30. Same as in Fig. 27 but for the $\bar{\nu}_{e}$-NC induced process on the deuteron. $\ldots \ldots \ldots 9$

31. Diagrams relevant for $\delta M$ and $Z_{N} \ldots \ldots \ldots \ldots \ldots \ldots \ldots \ldots \ldots \ldots \ldots \ldots \ldots \ldots \ldots$

32. Parts of a general diagram with the propagation of nucleons only. . . . . . . . 118

33. Diagrams relevant for the renormalization of $g_{A} / f_{\pi} \ldots \ldots \ldots \ldots \ldots \ldots \ldots \ldots \ldots$

34. Diagrams relevant for the renormalization of $f_{\pi} \ldots \ldots \ldots \ldots \ldots \ldots \ldots \ldots \ldots$

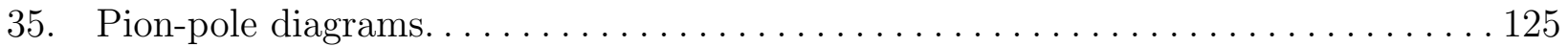

36. Time orderings for the pion-pole one-body axial current topologies, indicated in

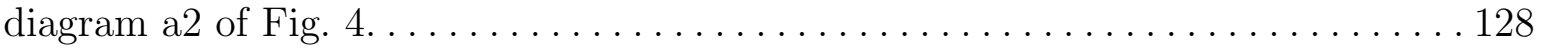


37. Feynman amplitudes contributing to the one-body axial charge at leading order. . 132 


\section{CHAPTER 1}

\section{INTRODUCTION}

The understanding of the structure and dynamics of nuclei has been a long-standing goal of nuclear physics. Typical binding energies are of order $8 \mathrm{MeV}$ per nucleon and Fermi momenta are around $1.35 \mathrm{fm}^{-1}$. Therefore nucleons are essentially non-relativistic and this justifies the conventional approach in which the nucleus is taken to consist of nucleons interacting with each other via two- and many-body effective potentials, and with external electroweak fields via one- and many-body effective currents. This picture has succeeded in describing satisfactorily many nuclear properties at a quantitative level (for a review, see Ref. [1]).

At a more fundamental level, nuclear interactions can be seen in terms of the interactions between the nucleon constituents, quarks and gluons. However the nonperturbative character of quantum chromodynamics (QCD) at low energies makes the problem of computing nuclear observables directly from QCD extremely difficult. One of the approaches used to study QCD in this non-perturbative regime is lattice QCD [2]. While lattice calculations (for light nuclei) have made and continue to make great progress, they are still in their infancy, and at this point in time are only able to provide a rather crude description of even the lightest nuclei (for a recent review see Ref. [3]).

Another approach that has been proven to be successful in the description of nuclear properties, and which we adopt in the present Thesis, is based on chiral effective field theory $(\chi \mathrm{EFT})$. In general, effective field theories are low-energy approximations to more fundamental theories. The general prescription for building an effective field theory requires writing down the most general Lagrangian consistent with all the symmetries of the underlying theory [4]. Each term in the Lagrangian is multiplied by a coefficient, known as low energy constant (LEC). A specific example of effective field theory is $\chi \mathrm{EFT}$ where, in its simplest formulation, nucleons and pions, rather than quarks and gluons, are the degrees of freedom. This framework was originally proposed by Weinberg in the early 1990's [5], and it has the great advantage of providing a direct connection between the symmetries of QCD and the nuclear forces. It also provides a practical calculational scheme that can, at least in principle, be improved systematically. 
During the last quarter century, $\chi \mathrm{EFT}$ has been used extensively to derive two- and many-nucleon interactions. After more than two decades of work, two-nucleon potentials have reached a precision close to that obtained by phenomenological representations $[5,6$, $7,8,9,10,11]$. This theoretical framework, based on the symmetries of QCD, has also been used to derive three- and four-nucleon forces consistently with the two-nucleon forces $[12,13,14,15]$. The application of these chiral forces in nuclei with $A \geq 3$ has just started, but is already leading to promising results in the description of few-nucleon observables [16].

Similarly, the interaction of nuclei with electroweak probes can be seen in terms of the interactions of quarks with electroweak gauge bosons (the massless photon and the massive $W^{ \pm}$and $Z^{0}$ ), but can also be described in $\chi$ EFT. Indeed, a number of few-nucleon reactions with external electroweak probes has been investigated in such an approach (for a review see Refs. [17, 18, 19] and references therein). Electromagnetic operators were first derived in a pioneering work of Park et al. [20], using covariant perturbation theory (Feynman diagrams). More recently, these electromagnetic currents have been derived by the JLabPisa group [21, 22, 23, 24] and, independently, by the Bochum-Bonn group in time ordered perturbation theory $[25,26]$, but with different prescriptions for isolating non iterative terms in reducible contributions. The resulting operators have been used to study a variety of electromagnetic observables, such as the charge and the magnetic radii and form factors of the deuteron and trinucleons $\left({ }^{3} \mathrm{He}\right.$ and $\left.{ }^{3} \mathrm{H}\right)$ [24, 27].

In this Thesis we focus on the weak axial charge and current operators. These were first obtained in $\chi \mathrm{EFT}$ in a paper by Park et al. [28], under a number of approximations (among others, near-threshold kinematics and ignoring altogether reducible diagrams). Here we adopt time ordered perturbation theory and further extend the formalism developed in Refs. [22, 23, 24]. We obtain an axial current that is finite and conserved in the limit of vanishing pion mass (known as the chiral limit). In contrast, loop corrections to the axial charge are divergent and we carry out a renormalization procedure, including a careful analysis of higher-order chiral Lagrangians and the construction of a complete set of contact terms for the axial charge.

The second objective of this Thesis is to apply these currents and study selected electroweak observables in few-nucleon processes, specifically tritium $\beta$-decay and neutrinodeuteron inclusive scattering. The tritium Gamow-Teller matrix element has been computed with trinucleon wave functions obtained from available two- and three-nucleon potentials corresponding to either $\chi$ EFT or meson-exchange phenomenology. The calculations of neutrinodeuteron cross sections at low energy have been carried out only in the $\chi$ EFT framework. 
The results obtained in this Thesis have confirmed those of previous phenomenological calculations that played a crucial role in the analysis and interpretation of the Sudbury Neutrino Observatory experiments [29]. These experiments established solar neutrino oscillations and the validity of the standard model for the generation of energy and neutrinos in the sun [30].

This thesis is organized as follows. In Chapter 2 we discuss the QCD Lagrangian and its symmetries - these are relevant for the construction of the chiral Lagrangians. Power counting is introduced and the chiral Lagrangians needed for the calculation of the weakaxial current are presented. In Chapter 3 the formalism used to derive nuclear potentials and electroweak currents is outlined. It is based on time ordered perturbation theory and allows us to isolate non iterative terms in reducible contributions in a systematic way. In Chapter 4 the well-known derivation of the two-nucleon potential up to one loop is sketched out. In Chapter 5 the derivation of the nuclear axial current and charge up to one loop is presented in considerable detail. After illustrating the calculation of the numerous diagrams, we show that important consistency checks of the calculation are satisfied (i.e., conservation of the current in the chiral limit, renormalization of the axial charge, etc.). In Chapter 6 this axial current is used to study tritium beta decay. In particular the use of nuclear wave functions derived from chiral potentials allows us to carry out a consistent $\chi \mathrm{EFT}$ calculation for the first time at high orders in the power counting. In Chapter 7 the axial current and charge obtained here, along with the previously derived electromagnetic current and charge, are employed to calculate the inclusive cross section for low energy neutrino-deuteron scattering. In Chapter 8 a summary of the results obtained is presented and possible future developments of this work are outlined. The appendices contain formal details. Appendices A and B report the explicit expressions of the chiral Lagrangians and corresponding interaction vertices needed for the calculations. Appendices C, D, E, and F contain details about the renormalization procedure adopted for the potential and currents. Appendix G deals with the construction of the contact axial charge operator. Appendix $\mathrm{H}$ illustrates nonstatic corrections for a class of diagrams relevant for the calculation of the axial current. Appendix I reports the regularized expressions for the loop contributions to the axial current. Appendix J discusses how the results in the one-body sector emerges in covariant perturbation theory. Lastly, Appendix K reports configuration-space expressions for the currents used in the tritium $\beta$-decay calculation. 


\section{CHAPTER 2}

\section{CHIRAL EFFECTIVE FIELD THEORY}

The nonperturbative nature of quantum chromodynamics (QCD) at low energies makes the task of performing ab initio (i.e., starting from the QCD Lagrangian) calculations of observables relevant for low-energy hadronic processes very difficult. One approach that has been successful in the description of low-energy processes is known as chiral effective field theory $(\chi \mathrm{EFT})$. Pions and nucleons (and low-energy excitations of the nucleon, such as the $\Delta$ isobar), rather than quarks and gluons, are the degrees of freedom of $\chi$ EFT. Chiral symmetry requires the pion to couple to these baryons, as well as to other pions, by powers of its momentum $Q$. As a consequence, classes of Lagrangians emerge, each characterized by a given power of $Q / \Lambda_{\chi}$, where $\Lambda_{\chi} \sim 1 \mathrm{GeV}$ is the chiral symmetry-breaking scale, or equivalently, a given order in the derivatives of the pion field and/or pion mass factors, and each containing a certain number of unknown parameters, so called low-energy constants (LECs). These LECs could in principle be calculated from the underlying QCD theory of quarks and gluons but, in practice, they are fixed by comparison with experimental data. They effectively encode short-range physics and the effects due to the excitation of baryon resonances, such as the $\Delta$ isobar, and heavy meson exchanges, not explicitly retained in the chiral Lagrangians. This approach was first developed to study pion-pion scattering in Refs. [4, 31], and later extended to study pion-nucleon scattering. In the next sections, after introducing chiral symmetry at the quark level, we illustrate how chiral pion-pion and pionnucleon Lagrangians emerge using the exact symmetries (Lorentz, parity, and time reversal) and the approximate chiral symmetry of QCD. In the following, we adopt the conventions of Ref. [32] in regards to the $\gamma$ matrices.

\subsection{CHIRAL SYMMETRY AND THE QCD LAGRANGIAN}

The QCD Lagrangian density in the two-flavor case of light up- and down-quarks is

$$
\begin{aligned}
\mathcal{L}_{Q C D} & =\sum_{f=u, d} \bar{f}(x)\left(i \gamma^{\mu} D_{\mu}-m_{f}\right) f(x)-\frac{1}{4} G_{\mu \nu, a}(x) G_{a}^{\mu \nu}(x) \\
& =\bar{q}(x)\left(i \gamma^{\mu} D_{\mu}-M\right) q(x)-\frac{1}{4} G_{\mu \nu, a}(x) G_{a}^{\mu \nu}(x)
\end{aligned}
$$


where

$$
D_{\mu}=\partial_{\mu}-i g G_{\mu}^{a}(x) \lambda_{a} / 2,
$$

where $\lambda_{a}$ with $a=1 \ldots 8$ are the $S U(3)_{\text {color }}$ Gell-Mann matrices (i.e., the generators of $S U(3)$ in the fundamental representation),

$$
G_{a}^{\mu \nu}(x)=\partial^{\mu} G_{a}^{\nu}(x)-\partial^{\nu} G_{a}^{\mu}(x),
$$

the gluon-field strength tensors, and $G_{a}^{\mu}(x)$ are the gluon spin-one fields. The sum over repeated color indices $a$ goes from 1 to 8 . Further, the quark fields $q(x)$ and the quark mass matrix $M$ are respectively given by

$$
q(x)=\left[\begin{array}{l}
u(x) \\
d(x)
\end{array}\right], \quad M=\left(\begin{array}{cc}
m_{u} & 0 \\
0 & m_{d}
\end{array}\right),
$$

where spinor and color indices are understood and $m_{u}$ and $m_{d}$ are the up- and down-quark masses. In Eq. (1), the $\theta$-term and gauge-fixing terms are not explicitly included, since they are not relevant in the present study. Having defined the right- and left-handed quark fields,

$$
\begin{aligned}
& q_{R}(x)=\frac{1+\gamma^{5}}{2} q(x)=\left[\begin{array}{l}
u_{R}(x) \\
d_{R}(x)
\end{array}\right], \\
& q_{L}(x)=\frac{1-\gamma^{5}}{2} q(x)=\left[\begin{array}{l}
u_{L}(x) \\
d_{L}(x)
\end{array}\right],
\end{aligned}
$$

we can write Eq. (1) as

$$
\begin{aligned}
\mathcal{L}_{Q C D}= & \bar{q}_{L}(x) i \gamma_{\mu} D^{\mu} q_{L}(x)+\bar{q}_{R}(x) i \gamma_{\mu} D^{\mu} q_{R}(x)-\bar{q}_{L}(x) M q_{R}(x)-\bar{q}_{R}(x) M q_{L}(x) \\
& -\frac{1}{4} G_{\mu \nu, a}(x) G_{a}^{\mu \nu}(x) .
\end{aligned}
$$

Left and right components of the quark fields are connected only through the mass matrix $M$. The light quark masses (in the $\overline{\mathrm{MS}}$ scheme at the scale $\mu=2 \mathrm{GeV}$ ) are [33]

$$
m_{u} \simeq(1.5-3.3) \mathrm{MeV}, \quad m_{d} \simeq(3.5-6.0) \mathrm{MeV} .
$$

These quark masses are significantly smaller than the typical hadronic masses, which (apart from the pion mass) are of the order of $1 \mathrm{GeV}$. Therefore when studying low energy QCD, as a starting point we consider the Lagrangian density in Eq. (1) in the limit of vanishing quark masses, known as the chiral limit,

$$
\mathcal{L}_{Q C D}^{0}=\bar{q}_{L}(x) i \gamma_{\mu} D^{\mu} q_{L}(x)+\bar{q}_{R}(x) i \gamma_{\mu} D^{\mu} q_{R}(x)-\frac{1}{4} G_{\mu \nu, a}(x) G_{a}^{\mu \nu}(x) .
$$


This Lagrangian is invariant under the following global transformations

$$
\begin{aligned}
U(1)_{R} \quad: \quad q_{R}(x) \rightarrow q_{R}^{\prime}(x)=e^{-i \Theta_{R}} q_{R}(x), \\
U(1)_{L} \quad: \quad q_{L}(x) \rightarrow q_{L}^{\prime}(x)=e^{-i \Theta_{L}} q_{L}(x), \\
S U(2)_{R} \quad: \quad q_{R}(x) \rightarrow q_{R}^{\prime}(x)=e^{-i \boldsymbol{\theta}_{R} \cdot \boldsymbol{\tau} / 2} q_{R}(x)=R q_{R}(x), \\
S U(2)_{L} \quad: \quad q_{L}(x) \rightarrow q_{L}^{\prime}(x)=e^{-i \boldsymbol{\theta}_{L} \cdot \boldsymbol{\tau} / 2} q_{L}(x)=L q_{L}(x),
\end{aligned}
$$

where $\boldsymbol{\tau}$ denotes the Pauli matrices in flavor space, $\Theta_{L, R}$ and $\boldsymbol{\theta}_{L, R}$ are, respectively, real numbers and three-dimensional real vectors, and the $2 \times 2$ matrices $R=e^{-i \boldsymbol{\theta}_{R} \cdot \boldsymbol{\tau} / 2}$ and $L=e^{-i \boldsymbol{\theta}_{L} \cdot \boldsymbol{\tau} / 2}$ have been defined. The symmetry group associated with the transformation laws in Eqs. (9)-(12) is denoted as $U(1)_{R} \otimes U(1)_{L} \otimes S U(2)_{R} \otimes S U(2)_{L}$, and can be shown [34] to be isomorphic to the group $G^{\prime}=U(1)_{V} \otimes U(1)_{A} \otimes S U(2)_{V} \otimes S U(2)_{A}$, defined as

$$
\begin{aligned}
U(1)_{V} & : q(x) \rightarrow q^{\prime}(x)=e^{-i \Theta_{V}} q(x), \\
U(1)_{A} & : \quad q(x) \rightarrow q^{\prime}(x)=e^{-i \gamma_{5} \Theta_{A}} q(x), \\
S U(2)_{V} & : \quad q(x) \rightarrow q^{\prime}(x)=e^{-i \boldsymbol{\theta}_{V} \cdot \boldsymbol{\tau} / 2} q(x)=V q(x), \\
S U(2)_{A} & : \quad q(x) \rightarrow q^{\prime}(x)=e^{-i \gamma_{5} \boldsymbol{\theta}_{A} \cdot \boldsymbol{\tau} / 2} q(x)=A q(x),
\end{aligned}
$$

where $\Theta_{V, A}$ and $\boldsymbol{\theta}_{V, A}$ are again real numbers and three-dimensional real vectors, respectively, with the $2 \times 2$ matrices $V$ and $A$ defined accordingly. We note that for $m_{u}=m_{d} \neq$ 0 the Lagrangian density in Eq. (1) is invariant under transformations of the subgroup $U(1)_{V} \otimes S U(2)_{V}$ of $G^{\prime}$. We also note that for each one of the eight generators of $G^{\prime}$ we obtain the following eight currents [34]

$$
\begin{aligned}
j_{\mu}(x) & =\bar{q}(x) \gamma_{\mu} q(x), \\
j_{\mu, 5}(x) & =\bar{q}(x) \gamma_{\mu} \gamma_{5} q(x), \\
\mathbf{j}_{\mu}(x) & =\frac{1}{2} \bar{q}(x) \gamma_{\mu} \boldsymbol{\tau} q(x), \\
\mathbf{j}_{\mu, 5}(x) & =\frac{1}{2} \bar{q}(x) \gamma_{\mu} \gamma_{5} \boldsymbol{\tau} q(x) .
\end{aligned}
$$

In the chiral limit, using Nöether's theorem, it can be shown [34] that these eight currents are conserved at the classical level. At the quantum level, the only classical symmetry belonging to the group $G^{\prime}$ that turns out to be broken is $U(1)_{A}$ [34]. The symmetry associated with $U(1)_{V}$ corresponds to quark number conservation (and it holds in the case of generic $M)$. The subgroup of $G^{\prime}$ relevant for the following discussion is $S U(2)_{V} \otimes S U(2)_{A}$, which we will denote as $G$ hereafter. In spite of the fact that Eq. (1) is approximately invariant 
under $G$, the ground state of QCD is symmetric only respect to its vector subgroup $S U(2)_{V}$ [34]. Therefore the classical symmetry associated with $G$ is said to be spontaneously broken into its vector subgroup $S U(2)_{V}$. There are various experimental facts behind this phenomenon. Hadrons in nature arrange themselves in almost mass degenerate multiplets of $S U(2)_{V}$, which implies that the vacuum of QCD (in the limit of vanishing quark masses) is invariant under $S U(2)_{V}$. If chiral symmetry had not been spontaneously broken, then we would have observed doublets of particles with opposite parity. However, no such doublets are observed in the low-energy hadronic spectrum. Another strong argument in favor of spontaneous symmetry breaking is the existence of pseudoscalar mesons (pions) that are natural candidates for the corresponding massless Nambu-Goldstone bosons whose existence is implied by Goldstone's theorem. However, pions are not exactly massless, but have a small mass due to the explicit chiral symmetry breaking by the nonvanishing quark masses. These arguments support the notion that the chiral group $G$ is spontaneously broken down to $S U(2)_{V}$.

In order to study the coupling to external probes in low energy QCD we promote, following Refs. [35, 36], the global symmetry described by $G$ to a local one. This is achieved by allowing the real parameters of Eqs. (13) to depend on space-time coordinates $x$, i.e., $\Theta_{V, A}(x)$ and $\boldsymbol{\theta}_{V, A}(x)$, and corresponding local vector and axial matrices $V(x)$ and $A(x)$. It can be shown [34] that, in order for $\mathcal{L}_{\mathrm{QCD}}$ to be invariant in the chiral limit under local transformations of $G$, it is necessary to introduce the following hermitian fields

$$
v_{\mu}(x)=\frac{\boldsymbol{\tau}}{2} \cdot \mathbf{V}_{\mu}(x), \quad a_{\mu}(x)=\frac{\boldsymbol{\tau}}{2} \cdot \mathbf{A}_{\mu}(x)
$$

It is also convenient to introduce the scalar $s(x)$ and pseudoscalar $p(x)$ hermitian fields, proportional to the identity matrix in isospin space, in order to reproduce the mass term that gives the explicit chiral symmetry breaking [34]. The following Lagrangian results $[35,36]$

$$
\begin{aligned}
\mathcal{L} & =\mathcal{L}_{Q C D}^{0}+\bar{q}(x) \gamma^{\mu}\left[v_{\mu}(x)+\gamma^{5} a_{\mu}(x)\right] q(x)-\bar{q}(x)\left[s(x)-i \gamma^{5} p(x)\right] q(x) \\
& =\mathcal{L}_{Q C D}^{0}+\mathcal{L}_{\text {ext }}
\end{aligned}
$$

where $\mathcal{L}_{Q C D}^{0}$ denotes $\mathcal{L}_{Q C D}$ in the chiral limit and $\mathcal{L}_{\text {ext }}$ denotes the Lagrangian depending on the external sources. We introduce the fields

$$
r_{\mu}(x)=v_{\mu}(x)+a_{\mu}(x), \quad l_{\mu}(x)=v_{\mu}(x)-a_{\mu}(x)
$$


and rewrite $\mathcal{L}$ in terms of left and right components of the quark fields obtaining for $\mathcal{L}_{\text {ext }}$

$$
\begin{aligned}
\mathcal{L}_{\mathrm{ext}}= & \bar{q}_{L}(x) \gamma^{\mu} l_{\mu}(x) q_{L}(x)+\bar{q}_{R}(x) \gamma^{\mu} r_{\mu}(x) q_{R}(x) \\
& -\bar{q}_{R}(x)[s(x)+i p(x)] q_{L}(x)-\bar{q}_{L}(x)[s(x)-i p(x)] q_{R}(x) .
\end{aligned}
$$

The invariance of $\mathcal{L}$ under local transformations of the chiral group $G$ is achieved if the external sources transform in the following way

$$
\begin{aligned}
r_{\mu}(x) & \rightarrow R(x) r_{\mu}(x) R^{\dagger}(x)+i R(x) \partial_{\mu} R^{\dagger}(x), \\
l_{\mu}(x) & \rightarrow L(x) l_{\mu}(x) L^{\dagger}(x)+i L(x) \partial_{\mu} L^{\dagger}(x), \\
s(x)+i p(x) & \rightarrow R(x)[s(x)+i p(x)] L^{\dagger}(x), \\
s(x)-i p(x) & \rightarrow L(x)[s(x)-i p(x)] R^{\dagger}(x),
\end{aligned}
$$

where the parameters that characterize the $R$ and $L$ transformations are now functions of $x$ in analogy with the $V(x)$ and $A(x)$ transformations defined previously. It is instructive to show how the coupling between quarks and the charged weak bosons $W_{\mu}^{ \pm}$arises from $\mathcal{L}$. This is important in the description of semileptonic weak decays such as pion decay $\pi^{-} \rightarrow \mu^{-} \bar{\nu}_{\mu}$ or neutron decay $n \rightarrow p e^{-} \bar{\nu}_{e}$. Choosing the external fields as

$$
r_{\mu}=0, \quad l_{\mu}=-\frac{g}{\sqrt{2}}\left(W_{\mu}^{+} T_{+}+\text {h.c. }\right)
$$

where h.c. refers to the hermitian conjugate, $g$ is the weak coupling related at lowest order of perturbation theory to the Fermi constant

$$
G_{F}=\sqrt{2} g^{2} /\left(8 M_{W}^{2}\right)
$$

and

$$
T_{+}=\left(\begin{array}{cc}
0 & V_{u d} \\
0 & 0
\end{array}\right),
$$

with $V_{u d}$ the Cabibbo-Kobayashi-Maskawa quark-mixing matrix element describing the transformation between two-flavor QCD eigenstates and the corresponding weak eigenstates. Therefore, inserting Eq. (23) into Eq. (18), we obtain

$$
\mathcal{L}_{\text {ext }}=-\frac{g}{2 \sqrt{2}}\left[W_{\mu}^{+} V_{u d} \bar{u} \gamma^{\mu}\left(1-\gamma_{5}\right) d+\text { h.c. }\right]
$$

namely the well-known electroweak Lagrangian that describes the coupling of up and down quarks to the massive charged weak bosons. 
Before proceeding any further, we remark that the external-source formalism has a deeper meaning than one described above. In quantum field theory the relevant quantities are Green's functions, which are vacuum expectation values of time-ordered products, and can be related to the physical scattering amplitude through the LSZ reduction formalism. Symmetries provide strong constraints on Green's functions, and among Green's functions. These relations are known as chiral Ward identities. In particular it has been shown in Ref. [37] that the infinite set of all chiral Ward identities satisfied by the Green's functions of QCD are equivalent, in the absence of anomalies, to an invariance of the generating functional under a local transformation of the external fields.

\subsection{EFFECTIVE LAGRANGIAN FOR PIONS}

We focus on energy scales small enough so that only pions need to be treated as explicit degrees of freedom, all other mesons can be integrated out in this limit. In the following $\pi_{a}$ denotes the pion field of isospin component $a$, while $\boldsymbol{\pi}$ the isotriplet of pion fields, and the dependence on the space-time coordinates $x$ is understood. We recall that QCD is invariant under the discrete symmetries (parity, time-reversal, and charge conjugation), the proper Lorentz group, and in the chiral limit, the chiral group $G$ introduced in the previous section. As first shown in Refs. [38, 39], the pion field $\pi_{a}$ transforms linearly under the subgroup $S U(2)_{V}$ of $G$ and nonlinearly under the full group $S U(2)_{V} \times S U(2)_{A}$. In particular, considering a generic parametrization $U$ (a matrix in isospin space) of the pion fields, it can be shown $[38,39]$ that under $S U(2)_{L} \times S U(2)_{R}$

$$
U \longrightarrow U^{\prime}=R U L^{\dagger}
$$

where $L$ and $R$ are the global transformations that have been defined in Eqs. (11)-(12). In general, we require $U$ to be unitary [34] with determinant equal to one, and assume it can be expanded in powers of $\pi_{a}$ as

$$
U=1+\frac{i}{f_{\pi}} \boldsymbol{\tau} \cdot \boldsymbol{\pi}-\frac{1}{2 f_{\pi}^{2}} \boldsymbol{\pi}^{2}-\frac{i \alpha}{f_{\pi}^{3}} \boldsymbol{\pi}^{2} \boldsymbol{\tau} \cdot \boldsymbol{\pi}+\frac{8 \alpha-1}{8 f_{\pi}^{4}} \boldsymbol{\pi}^{4}+\ldots
$$

where the first three terms of the expansion are fixed and the parameter $\alpha$ is arbitrary. Common choices are $\alpha=0$ and $\alpha=1 / 6$ corresponding, respectively, to the non-linear sigma model $U=(\sigma+i \boldsymbol{\tau} \cdot \boldsymbol{\pi}) / f_{\pi}$ with $\sigma=\sqrt{f_{\pi}^{2}-\boldsymbol{\pi}^{2}}$ and to the exponential parametrization $U=\exp \left(i \boldsymbol{\tau} \cdot \boldsymbol{\pi} / f_{\pi}\right)$, where $f_{\pi}$ is the pion decay constant whose value is $\simeq 92 \mathrm{MeV}$. We require the effective Lagrangian to have the same symmetry group of massless QCD, i.e., be invariant under Lorentz and chiral transformations. The building blocks are the matrices $U$ 
and $U^{\dagger}$, and four dimensional derivatives (denoted with $\partial_{\mu}$ ) of these quantities. In particular, Lorentz invariance requires that the number of derivatives to be even, so that the effective Lagrangian can be written as

$$
\mathcal{L}_{\pi \pi}=\mathcal{L}_{\pi \pi}^{(2)}+\mathcal{L}_{\pi \pi}^{(4)}+\cdots
$$

where the superscripts denote the power of $Q / \Lambda_{\chi}$ (we recall, for clarity, that $Q$ is the low momentum scale associated with incoming and outgoing pion momenta, and $\Lambda_{\chi} \sim 1 \mathrm{GeV}$ is the chiral symmetry-breaking scale). As an example, we discuss how the kinetic term of the leading order pion Lagrangian arises.

It is a simple exercise (see, for example, Ref. [34]) to show that the leading order (LO) Lagrangian is

$$
\mathcal{L}_{\pi \pi}^{(2)}=\frac{f_{\pi}^{2}}{4}\left\langle\partial_{\mu} U(x) \partial^{\mu} U^{\dagger}(x)\right\rangle
$$

where $f_{\pi}$ turns out to be the pion decay constant, and $\langle\cdots\rangle$ denotes the trace in the twoflavor space. It is worth noting that, as shown in Refs. [38, 39], all realizations of chiral symmetry are equivalent to each other modulo non linear field redefinitions of the following type

$$
\boldsymbol{\pi}^{\prime}=\boldsymbol{\pi} F[\boldsymbol{\pi}], \quad \text { with } \quad F[\mathbf{0}]=1
$$

According to Haag's theorem [40], such nonlinear field redefinitions do not affect the $S$ matrix elements. As a consequence $\pi \pi$ scattering amplitudes will be independent on the parametrization of the pion field (i.e., the $\alpha$ dependence in Eq. (27) must cancel out). It can be easily shown that, expanding $U$ in powers of the pion field, the LO Lagrangian assumes the following form

$$
\mathcal{L}_{\pi \pi}^{(2)}=\frac{1}{2} \partial_{\mu} \boldsymbol{\pi} \cdot \partial^{\mu} \boldsymbol{\pi}+\mathcal{O}\left(\pi^{4}\right)
$$

that corresponds to the kinetic term of a scalar field. It is clear now that the factor $f_{\pi}^{2} / 4$ in Eq. (29) has been chosen so as to reproduce the correct normalization for the kinetic term of the pion field. We are left with the problem that the Lagrangian in Eq. (29) describes interactions of massless pions, while the pions in the real world have a nonvanishing mass. Therefore, in order to introduce a nonvanishing mass term for the pion, linked to the explicit chiral symmetry breaking pattern present in the quark-level Lagrangian $\mathcal{L}_{Q C D}$, we observe that the mass term of $\mathcal{L}_{Q C D}$ would be invariant under chiral transformations if, instead of the matrix $M$, we would have a matrix $\widetilde{M}$ that under $S U(2)_{L} \times S U(2)_{R}$ would transform as

$$
\widetilde{M} \rightarrow \widetilde{M^{\prime}}=R \widetilde{M} L^{\dagger}
$$


So, using $\widetilde{M}$, we can build the most general Lagrangian invariant under the exact Lorentz, parity and charge conjugation symmetries, and chiral symmetry at first order in $\widetilde{M}$ as

$$
\mathcal{L}_{s . b .}=\frac{f_{\pi}^{2} B}{2}\left\langle\widetilde{M} U^{\dagger}(x)+U(x) \widetilde{M}^{\dagger}\right\rangle .
$$

We can now replace $\widetilde{M}$ by the quark mass matrix $M$, and notice that Eq. (33) has the same chiral-symmetry-breaking pattern of the quark mass term in Lagrangian of Eq. (6). Expanding Eq. (33) in powers of the pion field, we obtain

$$
\mathcal{L}_{s . b .}=f_{\pi}^{2} B\left(m_{u}+m_{d}\right)-\frac{B}{2}\left(m_{u}+m_{d}\right) \boldsymbol{\pi}^{2}+\mathcal{O}\left(\pi^{4}\right),
$$

where we require the second term to be the pion mass with $m_{\pi}^{2}=B\left(m_{u}+m_{d}\right)$. The first term consists of an irrelevant constant. It has been shown in Ref. [35] that the low energy constant $B$ is connected to the quark condensate $\langle 0|\bar{q} q| 0\rangle$ in the following way

$$
B=-\frac{1}{3 f_{\pi}^{2}}\langle 0|\bar{q} q| 0\rangle,
$$

where $|0\rangle$ denotes the full QCD vacuum. The quantity $B$ can be interpreted as providing a measure of the strength of the spontaneous symmetry breaking of the theory.

Similarly to what has been done in the previous section, in order to introduce couplings to external sources, we promote the global symmetry under $G$ to be a local one. Under local transformations, the pion field transforms as

$$
U \quad \rightarrow \quad U^{\prime}=L(x) U R^{\dagger}(x)
$$

We define the covariant derivative of the pion field as

$$
D_{\mu} U=\partial_{\mu} U-i r_{\mu}(x) U+i U l_{\mu}(x)
$$

where the external fields $r_{\mu}(x)$, and $l_{\mu}(x)$ have been introduced before. It is easy to show that

$$
D_{\mu} U \rightarrow R(x)\left(D_{\mu} U\right) L^{\dagger}(x)
$$

At leading order, besides $U$ and $U^{\dagger}$ and their derivatives, we can also use the fields $\chi(x)$ and $\chi^{\dagger}(x)$ defined as linear combinations of the fields $s(x)$ and $p(x)$

$$
\begin{aligned}
\chi & =2 B[s(x)+i p(x)], \\
\chi^{\dagger} & =2 B[s(x)-i p(x)],
\end{aligned}
$$


that transform accordingly to Eqs. (21) and (22), and the $x$-dependence of $\chi$ and $\chi^{\dagger}$ is understood. The parameter $B$ is defined as in Eq. (35). These building blocks of the chiral pion Lagrangian have the following chiral counting

$$
U \sim O\left(Q^{0}\right), \quad D_{\mu_{1}} \cdots D_{\mu_{n}} U \sim O\left(Q^{n}\right), \quad \chi \sim O\left(Q^{2}\right)
$$

At leading order in $Q^{2}$, the effective Lagrangian invariant under Lorentz, parity, charge conjugation and chiral transformations is now (see Ref. [34])

$$
\mathcal{L}_{\pi \pi}^{(2)}=\frac{f_{\pi}^{2}}{4}\left\langle D_{\mu} U\left(D^{\mu} U\right)^{\dagger}\right\rangle+\frac{f_{\pi}^{2}}{4}\left\langle\chi U^{\dagger}+U \chi^{\dagger}\right\rangle
$$

As we will see later, for the calculation of nuclear axial currents, both $\mathcal{L}_{\pi \pi}^{(2)}$ and $\mathcal{L}_{\pi \pi}^{(4)}$ are needed (see Appendix A.1 for the explicit expression of $\mathcal{L}_{\pi \pi}^{(4)}$ ).

\subsection{EFFECTIVE LAGRANGIANS FOR NUCLEONS AND PIONS}

We extend the previous treatment to include nucleons. In the following, $N$ denote the iso-doublet of nucleon fields, and it is convenient to define a matrix $u$ such that $u^{2}=U$ (the $x$-dependnce of $u$ and $U$ will be dropped, unless explicitly noted). Considering the transformation law of $U$ under global transformations $S U(2)_{L} \times S U(2)_{R}$, we obtain that $u$ transforms as [34]

$$
u \rightarrow u^{\prime}=R u h^{-1}(x)
$$

where $h(x)$ is defined as

$$
h(x)=[\sqrt{R U(x) L}]^{-1} R \sqrt{U(x)} .
$$

As first shown in Ref. [39], if we assume the nucleon field to transform under global transformations $S U(2)_{L} \otimes S U(2)_{R}$ as

$$
N \rightarrow N^{\prime}=h(x) N
$$

then the set $(U, N)$ defines a nonlinear realization of the group $G$, and therefore

$$
\left[\begin{array}{l}
U(x) \\
N(x)
\end{array}\right] \rightarrow\left[\begin{array}{l}
R U(x) L^{\dagger} \\
h(x) N(x)
\end{array}\right]
$$

In the case of a pure vector transformation $L=R=V$, from the definition of $u(x)$ it follows that $u^{\prime}(x)=V u(x) V^{\dagger}$, and therefore from Eq. (43) $h=V$, and the nucleonic field transforms 
coherently as an isospin doublet. However, the nucleon field transforms non-linearly under axial transformations [34].

The most general chiral-invariant Lagrangian for pions and nucleons can be constructed using building blocks, generically denoted as $O_{i}$, that transform under $S U(2)_{L} \times S U(2)_{R}$ as $O_{i}^{\prime}=h(x) O_{i} h^{-1}(x)$. The building blocks are $u, N$, and their derivatives. The derivative of the two-by-two matrix $u$ does not transform covariantly under the group $G$

$$
\partial_{\mu} u \rightarrow \partial_{\mu} u^{\prime}=R\left(\partial_{\mu} u\right) h^{-1}(x)+R u \partial_{\mu}\left[h^{-1}(x)\right] .
$$

However, if we define the following quantity $\widetilde{u}_{\mu}=i\left[u^{\dagger} \partial_{\mu} u-u \partial_{\mu} u^{\dagger}\right]$ we have

$$
\widetilde{u}_{\mu} \rightarrow \widetilde{u}_{\mu}^{\prime}=h(x) \widetilde{u}_{\mu} h^{-1}(x) .
$$

Similarly the derivative of the nucleon field $\partial_{\mu} N$ does not transform covariantly under global transformations of $G$

$$
\partial_{\mu} N \rightarrow \partial_{\mu} N^{\prime}=\partial_{\mu} h(x) N+h(x) \partial_{\mu} N .
$$

Therefore, in order to define a covariant derivative for the nucleon field, it is convenient to introduce the so called connection

$$
\widetilde{\Gamma}_{\mu}=\frac{1}{2}\left[u^{\dagger} \partial_{\mu} u+u \partial_{\mu} u^{\dagger}\right]
$$

which under the group $G$ transforms as

$$
\widetilde{\Gamma}_{\mu}^{\prime}=h(x) \widetilde{\Gamma}_{\mu} h(x)^{-1}
$$

Now we define the covariant derivative of the nucleon field as

$$
\widetilde{D}_{\mu}=\partial_{\mu}+\widetilde{\Gamma}_{\mu}
$$

and obtain under $G$

$$
\widetilde{D}_{\mu}^{\prime} N^{\prime}=h(x) \widetilde{D}_{\mu} N
$$

where the tilde serves to remind us that couplings to external sources have not yet been considered (i.e., the symmetry group $G$ is global). We can now build the most general Lagrangian that describes the couplings of the nucleons to pions. We will focus in the following on the leading order Lagrangian, denoted as $\widetilde{\mathcal{L}}_{\pi N}^{(1)}$. This Lagrangian must be invariant under Lorentz, parity and charge conjugation transformations, as well as transformations under 
the group $G$. We also require that in the limit of vanishing nucleon-pion couplings we should recover the usual Dirac equation for spin-1/2 fields. As a consequence $\widetilde{\mathcal{L}}_{\pi N}^{(1)}$ must be a bilinear in the nucleon fields and contain at most one derivative of the nucleon field. Using the building blocks $N, \widetilde{D}_{\mu} N$, and $\widetilde{u}_{\mu}$ it can be shown that $\widetilde{\mathcal{L}}_{\pi N}^{(1)}$ reads [34]

$$
\widetilde{\mathcal{L}}_{\pi N}^{(1)}=\bar{N}\left(i \gamma^{\mu} \widetilde{D}_{\mu}-m+\frac{g_{A}}{2} \gamma^{\mu} \gamma_{5} \widetilde{u}_{\mu}\right) N
$$

where $m$ denotes the nucleon mass, and $g_{A}$ is the nucleon axial coupling constant.

The coupling to external electroweak sources can be introduced in a very natural way in this framework by requiring that $\mathcal{L}_{\pi N}^{(1)}$ be invariant under local transformations of $G$ previously defined in Eq. (36). In analogy to the case of only pions we introduce the external fields $r_{\mu}$ and $l_{\mu}$ with the transformation laws given by Eqs.(19)-(20). Under the local group $G$ the set $U, N$ transforms as

$$
\left[\begin{array}{c}
U(x) \\
N(x)
\end{array}\right] \rightarrow\left[\begin{array}{c}
R(x) U(x) L^{\dagger}(x) \\
h(x) N(x)
\end{array}\right]
$$

Therefore it is convenient to define the two following quantities

$$
\begin{aligned}
u_{\mu} & =i\left[u^{\dagger}\left(\partial_{\mu}-i r_{\mu}\right) u-u\left(\partial_{\mu}-i l_{\mu}\right) u^{\dagger}\right], \\
\Gamma_{\mu} & =\frac{1}{2}\left[u^{\dagger}\left(\partial_{\mu}-i r_{\mu}\right) u+u\left(\partial_{\mu}-i l_{\mu}\right) u^{\dagger}\right]
\end{aligned}
$$

that represent generalizations of $\widetilde{u}_{\mu}$ and $\widetilde{\Gamma}_{\mu}$ to the case of local transformations. These last two quantities transform under local transformations as

$$
\begin{aligned}
& u_{\mu} \rightarrow u_{\mu}^{\prime}=h(x) u_{\mu} h^{-1}(x), \\
& \Gamma_{\mu} \rightarrow \Gamma_{\mu}^{\prime}=h(x) \Gamma_{\mu} h^{-1}(x),
\end{aligned}
$$

and therefore it is natural to define the following covariant derivative of the nucleon field

$$
D_{\mu} N=\left(\partial_{\mu}+\Gamma_{\mu}\right) N
$$

that is a generalization of the definiton in Eq. (52). Under the group $G$ we have

$$
D_{\mu}^{\prime} N^{\prime}=h(x) D_{\mu} N
$$

Therefore the most general $\pi N$ Lagrangian that describes the coupling to external sources, denoted in the following as $\mathcal{L}_{\pi N}^{(1)}$, it is a straightforward generalization of Eq. (54), where $\widetilde{D}_{\mu}$, and $\widetilde{u}_{\mu}$, have been replaced with $D_{\mu}$, and $u_{\mu}$, respectively,

$$
\mathcal{L}_{\pi N}^{(1)}=\bar{N}\left(i \gamma^{\mu} D_{\mu}-m+\frac{g_{A}}{2} \gamma^{\mu} \gamma_{5} u_{\mu}\right) N
$$


Higher order pion-nucleon chiral Lagrangians (with two or more insertions of $D_{\mu}$ and $u_{\mu}$ ) have been constructed in Ref. [41] and are reported in Appendix A. For the derivation of the nuclear axial currents the chiral Lagrangians $\mathcal{L}_{\pi N}^{(1)}, \mathcal{L}_{\pi N}^{(2)}$, and $\mathcal{L}_{\pi N}^{(3)}$ are needed. 


\subsection{CONTACT LAGRANGIANS}

In this Section we report contact Lagrangians (i.e., without exchange of pions) that contribute to the $N N$ scattering amplitude. These terms are well-known in the literature (see for example Ref. [5]), and have been built requiring invariance under Lorentz, parity, charge conjugation, and the chiral group $G$. The LO Lagrangian is given by

$$
\mathcal{L}_{N N}^{(0)}=-C_{S}(\bar{N} N)(\bar{N} N)-C_{T}\left(\bar{N} \gamma_{\mu} N\right)\left(\bar{N} \gamma^{\mu} N\right)
$$

and depends on two LECs denoted as $C_{S}$, and $C_{T}$. At order $Q$, since we have only one derivative of the nucleon field at our disposal, it is not possible to build a Lorentz scalar. The next nonvanishing order is $Q^{2}$ and the corresponding Lagrangian is denoted as $\mathcal{L}_{N N}^{(2)}$. It can be shown that there are twelve independent operator structures that contribute [42], involving two derivatives of the nucleon field. In the following we will not report the explicit expression of this Lagrangian (see Ref. [42]). 


\section{CHAPTER 3}

\section{NUCLEAR CHIRAL EFFECTIVE FIELD THEORY}

In this chapter we describe the general framework adopted to derive nuclear potentials and electroweak currents for use in studies of structure and low-energy electroweak transitions in nuclei. This framework is based on time-ordered perturbation theory (TOPT) formulated in terms of interaction Hamiltonians constructed from the $\pi \pi$ and $\pi N$ chiral Lagrangians in the canonical formalism (see Sec. 3.1). Because of the presence of bound states in $A \geq 2$ the derivation of the nuclear potentials and currents requires a careful analysis of the TOPT expansion, as illustrated in Sec. 3.3.

\subsection{INTERACTION HAMILTONIANS FROM CHIRAL LAGRANGIANS}

In this section we derive, in the canonical formalism, the interaction Hamiltonians implied by the $\pi \pi$ and $\pi N$ chiral Lagrangians of Ch. 2. These are combined as

$$
\mathcal{L}=\mathcal{L}_{\pi N}+\mathcal{L}_{\pi \pi}
$$

where

$$
\begin{aligned}
\mathcal{L}_{\pi N} & =\mathcal{L}_{\pi N}^{(1)}+\mathcal{L}_{\pi N}^{(2)}+\mathcal{L}_{\pi N}^{(3)}+\ldots, \\
\mathcal{L}_{\pi \pi} & =\mathcal{L}_{\pi \pi}^{(2)}+\mathcal{L}_{\pi \pi}^{(4)}+\ldots
\end{aligned}
$$

and the superscript $n$ specifies the chiral order $Q^{n}$, i.e., the number of derivatives of the pion field and/or insertions of the pion mass. External fields are counted as being of order $Q$. Since we are interested in deriving nuclear potentials and currents up to one loop, it suffices to retain in $\mathcal{L}$ up to $\mathcal{L}_{\pi N}^{(3)}$ and $\mathcal{L}_{\pi \pi}^{(4)}$. Further, since we are interested in axial currents, the right $r_{\mu}(x)$ and left $l_{\mu}(x)$ fields are taken as

$$
r_{\mu}(x)=-l_{\mu}(x)=\frac{1}{2} \boldsymbol{\tau} \cdot \mathbf{A}_{\mu}(x),
$$

where $A_{a}^{\mu}(x)$ is the external axial-vector field of isospin component $a$. The $x$-dependence of the various fields is dropped hereafter. As a consequence the total Lagrangian can be written in a compact form as

$$
\begin{aligned}
\mathcal{L}= & \bar{N}\left(i \not \partial-m+\Gamma_{a}^{0} \partial_{0} \pi_{a}+\Lambda_{a}^{i} \partial_{i} \pi_{a}+\Delta\right) N \\
& +\frac{1}{2}\left(\partial^{0} \pi_{a} G_{a b} \partial_{0} \pi_{b}+\partial^{i} \pi_{a} \widetilde{G}_{a b} \partial_{i} \pi_{b}-m_{\pi}^{2} \pi_{a} H_{a b} \pi_{b}\right)-f_{\pi} A_{a}^{\mu} F_{a b}\left(\partial_{\mu} \pi_{b}\right),
\end{aligned}
$$


where the symbols $\Gamma_{a}^{0}, \Lambda_{a}^{i}$, and $\Delta$ denote combinations of the pion and axial-vector fields (and their derivatives), having the following expansions

$$
\Gamma_{a}^{0}=\Gamma_{a}^{0}(0)+\Gamma_{a}^{0}(1)+\Gamma_{a}^{0}(2)
$$

and similarly for $\Lambda_{a}^{i}$, and

$$
\Delta=\Delta(1)+\Delta(2)+\Delta(3)
$$

where the argument $n$ in $\Gamma_{a}^{0}(n), \Lambda_{a}^{i}(n)$, and $\Delta(n)$ specifies the power counting $Q^{n}$. The symbols $G_{a b}, \widetilde{G}_{a b}, H_{a b}$, and $F_{a b}$ denote three-by-three matrices in isospin space, containing powers of the pion field and/or pion mass. A listing of all these quantities, limited to the terms relevant for the construction of the currents up to one loop, is provided in Appendix A. At this stage the various fields, masses, and coupling constants are to be understood as bare (un-renormalized) quantities.

From the Lagrangian $\mathcal{L}$ in Eq. (68) the conjugate momenta relative to the nucleon and pion fields follow as

$$
\begin{aligned}
\Pi^{\dagger} & =\frac{\partial \mathcal{L}}{\partial\left(\partial_{0} N\right)}=i \bar{N} \gamma^{0} \\
\Pi_{a} & =\frac{\partial \mathcal{L}}{\partial\left(\partial_{0} \pi_{a}\right)}=G_{a b} \partial^{0} \pi_{b}-f_{\pi} F_{a b} A_{b}^{0}+\bar{N} \Gamma_{a}^{0} N
\end{aligned}
$$

and the Hamiltonian then reads

$$
\mathcal{H}=\Pi^{\dagger} \partial_{0} N+\Pi_{a} \partial_{0} \pi_{a}-\mathcal{L}=\mathcal{H}_{0}+\mathcal{H}_{I}
$$

where $\mathcal{H}_{0}$,

$$
\mathcal{H}_{0}=\frac{1}{2}\left(\Pi_{a} \Pi_{a}-\partial^{i} \pi_{a} \partial_{i} \pi_{a}+m_{\pi}^{2} \pi_{a} \pi_{a}\right)+\bar{N}\left(-i \gamma^{i} \partial_{i}+m\right) N
$$

is the free pion and nucleon Hamiltonian, while $\mathcal{H}_{I}$ is the Hamiltonian accounting for the interactions between pions and nucleons as well as between these and the external field. We treat the latter in first order and therefore only keep terms linear in $\mathbf{A}_{\mu}$. The interaction Hamiltonian is then given by

$$
\begin{aligned}
\mathcal{H}_{I}= & \frac{1}{2} \Pi_{a}\left[\left(G^{-1}\right)_{a b}-\delta_{a b}\right] \Pi_{b}-\frac{1}{2}\left[\Pi_{a}\left(G^{-1}\right)_{a b}\left(\bar{N} \Gamma_{b}^{0} N\right)+\text { h.c. }\right] \\
& +\frac{f_{\pi}}{2}\left[\Pi_{a}\left(G^{-1}\right)_{a b} F_{b c} A_{c}^{0}+\text { h.c. }\right]-\frac{f_{\pi}}{2}\left[\left(\bar{N} \Gamma_{a}^{0} N\right)\left(G^{-1}\right)_{a b} F_{b c} A_{c}^{0}+\text { h.c. }\right] \\
& +\frac{1}{2}\left(\bar{N} \Gamma_{a}^{0} N\right)\left(G^{-1}\right)_{a b}\left(\bar{N} \Gamma_{b}^{0} N\right)-\bar{N}\left(\Lambda_{a}^{i} \partial_{i} \pi_{a}+\Delta\right) N \\
& -\frac{1}{2} \partial^{i} \pi_{a}\left(\widetilde{G}_{a b}-\delta_{a b}\right) \partial_{i} \pi_{b}+f_{\pi} A_{a}^{i} F_{a b} \partial_{i} \pi_{b}+\frac{m_{\pi}^{2}}{2} \pi_{a}\left(H_{a b}-\delta_{a b}\right) \pi_{b} .
\end{aligned}
$$


It admits the following expansion in powers of $Q$ :

$$
\mathcal{H}_{I}=\mathcal{H}_{I}^{(1)}+\mathcal{H}_{I}^{(2)}+\mathcal{H}_{I}^{(3)}+\ldots,
$$

and the vertices corresponding to the various interaction terms are listed in Appendix B. We quantize the theory by imposing the following relations at equal time

$$
\begin{aligned}
{\left[\pi_{a}(t, \mathbf{x}), \Pi_{b}(t, \mathbf{y})\right]_{-} } & =\delta_{a b} \delta(\mathbf{x}-\mathbf{y}) \\
{\left[N_{r}(t, \mathbf{x}), \Pi_{s}^{\dagger}(t, \mathbf{y})\right]_{+} } & =\delta_{r s} \delta(\mathbf{x}-\mathbf{y})
\end{aligned}
$$

where $[\ldots, \ldots]_{\mp}$ denote the commutator $(-)$ or anticommuatator $(+)$, and $r$ and $s$ denote the nucleonic spin-isospin indices.

\subsection{NUCLEAR POTENTIALS FROM FIELD THEORY AMPLITUDES}

Field theory amplitudes are evaluated in time-ordered perturbation theory (TOPT). Terms in this expansion are conveniently represented by diagrams. We distinguish between reducible diagrams (diagrams which involve at least one pure nucleonic intermediate state) and irreducible diagrams (diagrams which include pionic and nucleonic intermediate states). The former are enhanced with respect to the latter by a factor of $Q$ for each pure nucleonic intermediate state (see below). In the static limit - in the limit $m \rightarrow \infty$, i.e., neglecting nucleon kinetic energies - reducible contributions are divergent. The prescription proposed by Weinberg [5] to treat these is to define the nuclear potential and currents as given by the irreducible contributions only. Reducible contributions, instead, are generated by solving the Lippmann-Schwinger (or Schrödinger) equation with the nuclear potential (and currents) arising from irreducible amplitudes. As an aside, we note that, as first pointed out by Weinberg [5], in covariant perturbation theory reducible contributions are infrared divergent. In TOPT the infrared divergences are removed, and the problem becomes one of small energy denominators.

The formalism used here is based on this prescription [21]. However, the omission of reducible contributions from the definition of nuclear operators requires care, when the irreducible amplitudes are evaluated in the static approximation. The iterative process will, in that limit, generate only part of the reducible amplitude. The reducible part of the amplitude beyond the static approximation needs to be incorporated order by order-along with the irreducible amplitude - in the definition of nuclear operators. This scheme in combination with TOPT, which is best suited to separate the reducible content from the irreducible one, 
has been implemented in Refs. [22, 23, 24] and is described below. The method does lead to nuclear operators, which are not uniquely defined because of the non-uniqueness of the transition amplitude off-the-energy-shell. This lack of uniqueness is unavoidable; however, since the resulting operators are unitarily equivalent, the description of physical observables is not affected by this ambiguity [23, 43].

Another approach for overcoming the difficulties posed by the reducible amplitudes has been introduced by Epelbaum and collaborators [44]. That method is usually referred to as the unitary transformation method, and is based on TOPT too. It exploits the Okubo (unitary) transformation [45] to decouple the Fock space of pions and nucleons into two subspaces, one that has pure nucleonic states and the other with states which retain at least one pion. In this decoupled space, the amplitude does not involve enhanced contributions associated with the reducible diagrams. The subspaces are not uniquely defined since it is always possible to perform additional unitary transformations on them, with a consequent change in the formal definition of the resulting nuclear operators. This, of course, does not affect physical representations.

The two TOPT-based methods outlined above lead to formally equivalent operator structures for the nuclear potential up to loop corrections included. It is plausible that the two methods are closely related, although this remains to be proved. Below we briefly outline the method described in Refs. [22, 23, 24], and sketch how nuclear potentials are obtained. In the next section we extend the present method to the case of electroweak currents.

The starting point is the conventional perturbative expansion for the two-nucleon $(N N)$ amplitude

$$
\langle f|T| i\rangle=\left\langle f\left|H_{I} \sum_{n=1}^{\infty}\left(\frac{1}{E_{i}-H_{0}+i \eta} H_{I}\right)^{n-1}\right| i\right\rangle .
$$

Here $|i\rangle$ and $|f\rangle$ represent the initial and final states, respectively $\left|N_{1} N_{2}\right\rangle$ and $\left|N_{1}^{\prime} N_{2}^{\prime}\right\rangle$ of energies $E_{i}$ and $E_{f}$ with $E_{i}=E_{f}, H_{0}$ is the Hamiltonian describing free pions and nucleons, and $H_{I}$ is the Hamiltonian describing interactions among these particles,

$$
H_{0}=\int \mathrm{d} \mathbf{x} \mathcal{H}_{0}(\mathbf{x})
$$

and similarly for $H_{I}$, where the Hamiltonian $\mathcal{H}_{0}$ and $\mathcal{H}_{I}$ are defined as in Sec. 3.1 with the various fields taken in the Schrödinger picture. The evaluation of the amplitude above is carried out in practice by inserting complete sets of $H_{0}$ eigenstates between successive terms of $H_{I}$. Power counting is then used to organize the expansion in powers of $Q / \Lambda_{\chi} \ll 1$.

In the perturbative series, Eq. (79), a generic (reducible or irreducible) contribution is characterized by a certain number, say $N$, of vertices, each scaling as $Q^{\alpha_{i}} \times Q^{-\beta_{i} / 2}$ 
$(i=1, \ldots, N)$, where $\alpha_{i}$ is the power counting implied by the relevant interaction Hamiltonian $H_{I}$ and $\beta_{i}$ is the number of pions in and/or out of the vertex, a corresponding $N-1$ number of energy denominators, and $L$ loops. Out of these $N-1$ energy denominators, $N_{K}$ of them will involve only nucleon kinetic energies which scale as $Q^{2}$, and the remaining $N-N_{K}-1$ energy denominators will involve, in addition, pion energies, which are of order $Q$. Loops, on the other hand, contribute a factor $Q^{3}$ each, since they imply integrations over intermediate three momenta. Hence the power counting associated with such a contribution is

$$
\left(\prod_{i=1}^{N} Q^{\alpha_{i}-\beta_{i} / 2}\right) \times\left[Q^{-\left(N-N_{K}-1\right)} Q^{-2 N_{K}}\right] \times Q^{3 L} .
$$

Clearly, each of the $N-N_{K}-1$ energy denominators can be further expanded as

$$
\frac{1}{E_{i}-E_{I}-\omega_{\pi}}=-\frac{1}{\omega_{\pi}}\left[1+\frac{E_{i}-E_{I}}{\omega_{\pi}}+\frac{\left(E_{i}-E_{I}\right)^{2}}{\omega_{\pi}^{2}}+\ldots\right]
$$

where $E_{I}$ denotes the energy of the intermediate state (including the kinetic energies of the two nucleons), and $\omega_{\pi}$ the pion energy (or energies, as the case may be) - the ratio $\left(E_{i}-E_{I}\right) / \omega_{\pi}$ is of order $Q$. The leading order term $-1 / \omega_{\pi}$ represents the static limit, while the sub-leading terms involving powers of $\left(E_{i}-E_{I}\right) / \omega_{\pi}$ represent non-static corrections of increasing order, i.e. recoil corrections.

The $Q$-scaling of the interaction vertices and the considerations above show that $T$ admits the following expansion

$$
T=T^{(\nu)}+T^{(\nu+1)}+T^{(\nu+2)}+\ldots
$$

where $T^{(n)} \sim Q^{n}$, and chiral symmetry ensures that $\nu$ is finite. In the case of the two-nucleon amplitude $\nu=0$. A two-nucleon potential $v$ can then be derived which, when iterated into the Lippmann-Schwinger (LS) equation

$$
v+v G_{0} v+v G_{0} v G_{0} v+\ldots
$$

leads to the $T$-matrix on-the-energy-shell $\left(E_{i}=E_{f}\right)$ of Eq. (83) and order by order in the power counting. In practice, this requirement can only be satisfied up to a certain order $n^{*}$, and the resulting potential, when inserted in the LS equation, will generate contributions of order $n>n^{*}$, which do not match $T^{(n)}$. In Eq. (84), $G_{0}=1 /\left(E_{i}-E_{I}+i \eta\right)$ denotes the free two-nucleon propagator, and we assume that

$$
v=v^{(0)}+v^{(1)}+v^{(2)}+\ldots
$$


where the still to be determined $v^{(n)}$ is of order $Q^{n}$. We note that, generally, a term like $v^{(m)} G_{0} v^{(n)}$ is of order $Q^{m+n+1}$, since $G_{0}$ is of order $Q^{-2}$ and the implicit loop integration brings in a factor $Q^{3}$.

Having established the above power counting, we obtain

$$
\begin{aligned}
v^{(0)}=T^{(0)} & , \\
v^{(1)}=T^{(1)} & -\left[v^{(0)} G_{0} v^{(0)}\right], \\
v^{(2)}=T^{(2)} & -\left[v^{(0)} G_{0} v^{(0)} G_{0} v^{(0)}\right] \\
& -\left[v^{(1)} G_{0} v^{(0)}+v^{(0)} G_{0} v^{(1)}\right], \\
v^{(3)}=T^{(3)} & -\left[v^{(0)} G_{0} v^{(0)} G_{0} v^{(0)} G_{0} v^{(0)}\right] \\
& -\left[v^{(1)} G_{0} v^{(0)} G_{0} v^{(0)}+\text { permutations }\right] \\
& -\left[v^{(2)} G_{0} v^{(0)}+v^{(0)} G_{0} v^{(2)}\right] \\
& -\left[v^{(1)} G_{0} v^{(1)}\right],
\end{aligned}
$$

and so on. The potentials $v^{(n)}$ are discussed in the next chapter. In particular, it will be shown that $v^{(1)}$ vanishes identically, and the first nonvanishing correction is suppressed by two powers of $Q$ relative to the leading order.

\subsection{NUCLEAR CURRENTS FROM FIELD THEORY AMPLITUDES}

The inclusion (in first order) of electroweak interactions in the perturbative expansion of Eq. (79) is in principle straightforward. The transition amplitude can be expanded as

$$
T_{e}=T_{e}^{\left(\nu_{e}\right)}+T_{e}^{\left(\nu_{e}+1\right)}+T_{e}^{\left(\nu_{e}+2\right)}+\ldots,
$$

where $T_{e}^{(n)}$ is of order $Q^{n}$ and $\nu_{e}=-3$ in this case. The strong interaction potential derived in the previous section is denoted by $v$, and the potential describing the interaction with the external fields by $v_{e}$. In the electromagnetic case $v_{e} \rightarrow v_{\gamma}=V^{0} \rho_{\gamma}-\mathbf{V} \cdot \mathbf{j}_{\gamma}$, while in the weak axial case $v_{e} \rightarrow v_{5}=A_{a}^{0} \rho_{5, a}-\mathbf{A}_{5, a} \cdot \mathbf{j}_{5, a}$, where $\rho_{\gamma}\left(\rho_{5}\right)$ and $\mathbf{j} \gamma\left(\mathbf{j}_{5, a}\right)$ are the electromagnetic (weak axial) charge and current, and $V^{\mu}=\left(V^{0}, \mathbf{V}\right)\left[A^{\mu}=\left(A^{0}, \mathbf{A}\right)\right]$ is the electromagnetic (weak axial) field.

The requirement that in the context of the LS equation,

$$
\left(v+v_{e}\right)+\left(v+v_{e}\right) G_{0}\left(v+v_{e}\right)+\left(v+v_{e}\right) G_{0}\left(v+v_{e}\right) G_{0}\left(v+v_{e}\right)+\ldots,
$$

matches $T_{5}$ order by order in the power counting implies relations for the $v_{\gamma}^{(n)}=V^{0} \rho_{\gamma}^{(n)}-$ 
$\mathbf{V} \cdot \mathbf{j}_{\gamma}^{(n)}$ and $v_{5}^{(n)}=A_{a}^{0} \rho_{5, a}^{(n)}-\mathbf{A}_{5, a} \cdot \mathbf{j}_{5, a}^{(n)}$ similar to those derived earlier $[23]$

$$
\begin{aligned}
& v_{e}^{(-3)}=T_{e}^{(-3)} \\
& v_{e}^{(-2)}=T_{e}^{(-2)}-\left[v_{e}^{(-3)} G_{0} v^{(0)}+v^{(0)} G_{0} v_{e}^{(-3)}\right], \\
& v_{e}^{(-1)}=T_{e}^{(-1)}-\left[v_{e}^{(-3)} G_{0} v^{(0)} G_{0} v^{(0)}+\text { permutations }\right] \\
& -\left[v_{e}^{(-2)} G_{0} v^{(0)}+v^{(0)} G_{0} v_{e}^{(-2)}\right] \text {, } \\
& v_{e}^{(0)}=T_{e}^{(0)}-\left[v_{e}^{(-3)} G_{0} v^{(0)} G_{0} v^{(0)} G_{0} v^{(0)}+\text { permutations }\right] \\
& -\left[v_{e}^{(-2)} G_{0} v^{(0)} G_{0} v^{(0)}+\text { permutations }\right] \\
& -\left[v_{e}^{(-1)} G_{0} v^{(0)}+v^{(0)} G_{0} v_{e}^{(-1)}\right] \\
& -\left[v_{e}^{(-3)} G_{0} v^{(2)}+v^{(2)} G_{0} v_{e}^{(-3)}\right] \text {, } \\
& v_{e}^{(1)}=T_{e}^{(1)}-\left[v_{e}^{(-3)} G_{0} v^{(0)} G_{0} v^{(0)} G_{0} v^{(0)} G_{0} v^{(0)}+\text { permutations }\right] \\
& -\left[v_{e}^{(-2)} G_{0} v^{(0)} G_{0} v^{(0)} G_{0} v^{(0)}+\text { permutations }\right] \\
& -\left[v_{e}^{(-1)} G_{0} v^{(0)} G_{0} v^{(0)}+\text { permutations }\right] \\
& -\left[v_{e}^{(0)} G_{0} v^{(0)}+v^{(0)} G_{0} v_{e}^{(0)}\right] \\
& -\left[v_{e}^{(-3)} G_{0} v^{(2)} G_{0} v^{(0)}+\text { permutations }\right] \\
& -\left[v_{e}^{(-2)} G_{0} v^{(2)}+v^{(2)} G_{0} v_{e}^{(-2)}\right] \\
& -\left[v_{e}^{(-3)} G_{0} v^{(3)}+v^{(3)} G_{0} v_{e}^{(-3)}\right] \text {, }
\end{aligned}
$$

where we have made use of the fact that $v^{(1)}=0$ (see Ch. 4), and have only retained terms linear in $v_{e}$. Relations in Eqs. (86)-(89), and those above allow us to construct $v^{(n)}$ and $v_{e}^{(n)}$ from $T^{(n)}$ and $T_{e}^{(n)}$. 


\section{CHAPTER 4}

\section{CHIRAL POTENTIAL AT ONE LOOP}

In this Chapter we report a derivation of the chiral two-nucleon $(N N)$ potential up to next-to-next-to-leading order $\mathrm{N}^{2} \mathrm{LO}$ (order $Q^{2}$ ), along with some contributions at $\mathrm{N}^{3} \mathrm{LO}$ (order $Q^{3}$ ) that will turn out to be relevant for the derivation of the nuclear axial current up to one loop. The $N N$ potential up to one loop can be symbolically expressed as

$$
v=v_{\mathrm{OPE}}+v_{\mathrm{TPE}}+v_{\mathrm{CT}},
$$

namely as a sum of terms due to one-pion exchange (OPE), two-pion exchange (TPE), and contact (CT) contributions. We note here that TPE contributions (at order $Q^{2}$ ) were first derived by Van Kolck and collaborators [6, 46]. Later, Kaiser and collaborators rederived TPE contributions as well as loop corrections to OPE (up to order $Q^{2}$ ) using covariant perturbation theory (see Ref. [7]). Soon after, Epelbaum and collaborators obtained these TPE contributions and loop corrections to OPE and contact interactions within the unitary transformation method in Ref. [8].

\subsection{STATIC POTENTIAL UP TO ORDER $Q^{2}$}

In Fig. 1 we show the diagrams illustrating the contributions occurring up to $\mathrm{N}^{2} \mathrm{LO}$ (order $\left.Q^{2}\right)$.
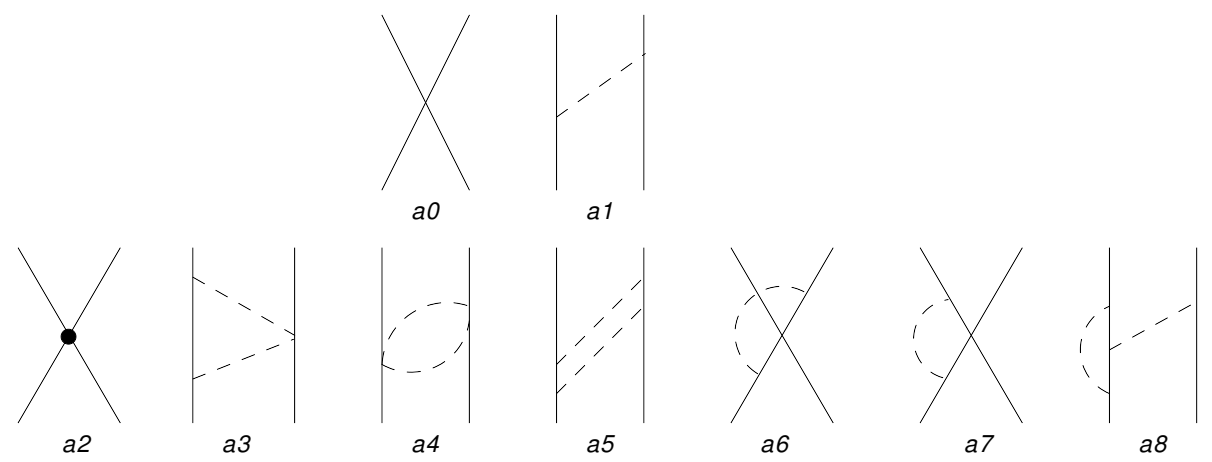

FIG. 1: Diagrams illustrating the contribution to the $N N$ potential entering at LO $Q^{0}$, panels a0, and a1, and $\mathrm{N}^{2} \mathrm{LO}\left(Q^{2}\right)$, panels a2-a8. Nucleons and pions are denoted by solid and dashed lines, respectively. The filled circle in panel a2 represents the vertex from contact Hamiltonians containing two gradients of the nucleons' fields. Only one among the possible time orderings is shown for each topology. 
At LO $\left(Q^{0}\right)$ there is a contact interaction, panel a0, along with the one-pion-exchange (OPE) contribution, panel a1. The next static contributions enter at $\mathrm{N}^{2} \mathrm{LO}$, and we distinguish among three different categories: (i) contact interactions involving two gradients acting on the nucleons' fields, panel a2; (ii) TPE diagrams, panels a3-a5; (iii) loop corrections to the LO contact and OPE interactions, panels a6-a8. Time ordered diagrams of the type illustrated by panels a1-a4 are irreducible, while those of the type illustrated by panels a5-a8 have both reducible and irreducible contributions. Here we limit ourselves to report the final expressions of the various contributions, and show how divergencies are reabasorbed through a redefinition of the contact terms at order $Q^{2}$ (for a detailed discussion see Ref. [22]). The renormalization of loop corrections to the OPE will be discussed in the next section (diagram a8).

\subsubsection{FORMAL EXPRESSIONS}

In what follows we use the notation introduced in Ref. [21]. In particular, the potential is obtained in the center-of-mass frame where the nucleon initial and final relative momenta are $\mathbf{p}$ and $\mathbf{p}^{\prime}$, respectively. We also define $\mathbf{k}=\mathbf{p}^{\prime}-\mathbf{p}, \mathbf{K}=\left(\mathbf{p}^{\prime}+\mathbf{p}\right) / 2$, and $\omega_{k}=\sqrt{k^{2}+m_{\pi}^{2}}$. An overall momentum-conserving $\delta$-function is understood in all terms listed in this chapter. The diagram illustrated by panel a0 gives rise to the LO contact potential, which is expressed in terms of the two LEC's $C_{S}$ and $C_{T}$ as

$$
v_{\mathrm{CT}}^{(0)}=C_{S}+C_{T} \boldsymbol{\sigma}_{1} \cdot \boldsymbol{\sigma}_{2}
$$

while that of panel a1 leads to the standard OPE potential,

$$
v_{\mathrm{OPE}}^{(0)}=-\frac{g_{A}^{2}}{4 f_{\pi}^{2}} \boldsymbol{\tau}_{1} \cdot \boldsymbol{\tau}_{2} \frac{\boldsymbol{\sigma}_{1} \cdot \mathbf{k} \boldsymbol{\sigma}_{2} \cdot \mathbf{k}}{\omega_{k}^{2}} .
$$

Next we consider the contributions arising from panel a2 of Fig. 1, which lead to

$$
\begin{aligned}
v_{\mathrm{CT}}^{(2)} & =C_{1} k^{2}+C_{2} K^{2}+\left(C_{3} k^{2}+C_{4} K^{2}\right) \boldsymbol{\sigma}_{1} \cdot \boldsymbol{\sigma}_{2}+i C_{5} \frac{\boldsymbol{\sigma}_{1}+\boldsymbol{\sigma}_{2}}{2} \cdot \mathbf{K} \times \mathbf{k} \\
& +C_{6} \boldsymbol{\sigma}_{1} \cdot \mathbf{k} \boldsymbol{\sigma}_{2} \cdot \mathbf{k}+C_{7} \boldsymbol{\sigma}_{1} \cdot \mathbf{K} \boldsymbol{\sigma}_{2} \cdot \mathbf{K} .
\end{aligned}
$$

Two-pion-exchange contributions are given by diagrams in panel a3-a5, and read

$$
\begin{aligned}
\widetilde{v}_{\mathrm{TPE}}^{(2)}=v^{(2)}(\mathrm{a} 3+\mathrm{a} 4+\mathrm{a} 5)=- & \frac{1}{128 f_{\pi}^{4}} \boldsymbol{\tau}_{1} \cdot \boldsymbol{\tau}_{2}\left[8 g_{A}^{2}\left[I^{(2)}(k)-k^{2} I^{(0)}(k)\right]+4 g_{A}^{4}\left[J^{(4)}(k)\right.\right. \\
& \left.\left.-2 k^{2} J^{(2)}(k)+k^{4} J^{(0)}(k)\right]+L(k)\right] \\
& -\frac{3 g_{A}^{4}}{16 f_{\pi}^{4}}\left(\boldsymbol{\sigma}_{1} \times \mathbf{k}\right)_{i}\left(\boldsymbol{\sigma}_{2} \times \mathbf{k}\right)_{j} J_{i j}^{(2)}(k)
\end{aligned}
$$


where we have defined the following loop functions

$$
\begin{aligned}
I^{(0)}(k) & =\int \frac{d \mathbf{p}}{(2 \pi)^{3}} f\left(\omega_{-}, \omega_{+}\right), \\
I^{(2)}(k) & =\int \frac{d \mathbf{p}}{(2 \pi)^{3}} p^{2} f\left(\omega_{-}, \omega_{+}\right), \\
J^{(0)}(k) & =\int \frac{d \mathbf{p}}{(2 \pi)^{3}} g\left(\omega_{+}, \omega_{-}\right), \\
J^{(2)}(k) & =\int \frac{d \mathbf{p}}{(2 \pi)^{3}} p^{2} g\left(\omega_{+}, \omega_{-}\right), \\
J^{(4)}(k) & =\int \frac{d \mathbf{p}}{(2 \pi)^{3}} p^{4} g\left(\omega_{+}, \omega_{-}\right), \\
J_{i j}^{(2)}(\mathbf{k}) & =\int \frac{d \mathbf{p}}{(2 \pi)^{3}} p_{i} p_{j} g\left(\omega_{+}, \omega_{-}\right), \\
L(k) & =\int \frac{d \mathbf{p}}{(2 \pi)^{3}}\left(\omega_{+}-\omega_{-}\right)^{2} f\left(\omega_{-}, \omega_{+}\right),
\end{aligned}
$$

with

$$
\begin{aligned}
& f\left(\omega_{-}, \omega_{+}\right)=\frac{1}{\omega_{+} \omega_{-}\left(\omega_{+}+\omega_{-}\right)}, \\
& g\left(\omega_{-}, \omega_{+}\right)=\frac{\omega_{+}^{2}+\omega_{+} \omega_{-}+\omega_{-}^{2}}{\omega_{+}^{3} \omega_{-}^{3}\left(\omega_{+}+\omega_{-}\right)},
\end{aligned}
$$

and

$$
\omega_{ \pm}=\sqrt{(\mathbf{p} \pm \mathbf{k})^{2}+4 m_{\pi}^{2}} .
$$

These loop functions are ultraviolet divergent, and we use dimensional regularization to isolate the corresponding divergencies. Expressions of the regularized kernels are reported in Appendix B of Ref. [22]. After dimensional regularization the two-pion exchange contribution can be written in the following compact form

$$
\begin{aligned}
\widetilde{v}_{\mathrm{TPE}}^{(2)}= & v_{\mathrm{TPE}}^{(2)}+\boldsymbol{\tau}_{1} \cdot \boldsymbol{\tau}_{2} P_{2}(k) \\
& +\left(k^{2} \boldsymbol{\sigma}_{1} \cdot \boldsymbol{\sigma}_{2}-\boldsymbol{\sigma}_{1} \cdot \mathbf{k} \boldsymbol{\sigma}_{2} \cdot \mathbf{k}\right) P_{0},
\end{aligned}
$$

where the renormalized finite part is

$$
\begin{aligned}
v_{\mathrm{TPE}}^{(2)}= & \frac{1}{768 \pi^{2} f_{\pi}^{4}} \boldsymbol{\tau}_{1} \cdot \boldsymbol{\tau}_{2} G(k)\left[4 m_{\pi}^{2}\left(1+4 g_{A}^{2}-5 g_{A}^{4}\right)\right. \\
& \left.+k^{2}\left(1+10 g_{A}^{2}-23 g_{A}^{4}\right)-\frac{48 g_{A}^{4} m_{\pi}^{4}}{s^{2}}\right] \\
& +\frac{3 g_{A}^{4}}{128 \pi^{2} f_{\pi}^{4}}\left(k^{2} \boldsymbol{\sigma}_{1} \cdot \boldsymbol{\sigma}_{2}-\boldsymbol{\sigma}_{1} \cdot \mathbf{k}_{2} \boldsymbol{\sigma}_{2} \cdot \mathbf{k}\right) .
\end{aligned}
$$


Here we have defined

$$
G(k)=\frac{s}{k} \ln \left(\frac{s+k}{s-k}\right)
$$

with $s=\sqrt{4 m_{\pi}^{2}+k^{2}}$. The divergencies are lumped into the polynomials $P_{2}(k)$, and $P_{0}$

$$
\begin{gathered}
P_{2}(k)=-\frac{1}{384 \pi^{2} f_{\pi}^{4}}\left[m_{\pi}^{2}\left[4+4 g_{A}^{2}+16 g_{A}^{4}-9 g_{A}^{2}\left(2-5 g_{A}^{2}\right) d_{\epsilon}\right]\right. \\
\left.+\frac{4}{3} k^{2}\left[\frac{5}{8}+\frac{13}{4} g_{A}^{2}-\frac{3}{8} g_{A}^{4}-\frac{3}{8}\left(1+10 g_{A}^{2}-23 g_{A}^{4}\right) d_{\epsilon}\right]\right] \\
P_{0}=\frac{3 g_{A}^{4}}{128 \pi^{2} f_{\pi}^{4}}\left(d_{\epsilon}-\frac{1}{3}\right),
\end{gathered}
$$

with the constant $d_{\epsilon}$ defined as

$$
d_{\epsilon}=-\frac{2}{\epsilon}+\gamma-\ln 4 \pi+\ln \frac{m_{\pi}^{2}}{\mu^{2}}-1
$$

where $\epsilon=3-d$ ( $d$ is the number of dimensions $), \gamma$ is Euler's constant, and $\mu$ is a renormalization scale.

The contributions of diagrams a6 and a7 of Fig. 1 are given by

$$
v^{(2)}(\mathrm{a} 6+\mathrm{a} 7)=\frac{g_{A}^{2}}{3 f_{\pi}^{2}} C_{T} \boldsymbol{\tau}_{1} \cdot \boldsymbol{\tau}_{2} \boldsymbol{\sigma}_{1} \cdot \boldsymbol{\sigma}_{2} J_{13}-\frac{g_{A}^{2}}{f_{\pi}^{2}} C_{T} \boldsymbol{\sigma}_{1} \cdot \boldsymbol{\sigma}_{2} J_{13} .
$$

where the quantity $J_{13}$, defined in Eq. (392), is ultraviolet divergent and reads (after dimensional regularization)

$$
J_{13}=\frac{3 m_{\pi}^{2}}{8 \pi^{2}}\left(d_{\epsilon}-\frac{7}{3}\right)
$$

Note that diagrams a6 and a7 include reducible contributions. The partial cancellation between these and contributions resulting from iterations of the LS equation has been taken into account. Finally following Ref. [22] we note that Fierz identities allow us to rewrite the spin-isospin structures in Eq. (105) and (111) as

$$
\begin{array}{r}
\boldsymbol{\tau}_{1} \cdot \boldsymbol{\tau}_{2} \longrightarrow-2-\boldsymbol{\sigma}_{1} \cdot \boldsymbol{\sigma}_{2}, \\
\boldsymbol{\tau}_{1} \cdot \boldsymbol{\tau}_{2} k^{2} \longrightarrow-4\left(1+\boldsymbol{\sigma}_{1} \cdot \boldsymbol{\sigma}_{2}\right) K^{2}-k^{2}, \\
\boldsymbol{\tau}_{1} \cdot \boldsymbol{\tau}_{2} \boldsymbol{\sigma}_{1} \cdot \boldsymbol{\sigma}_{2} \longrightarrow-3
\end{array}
$$


Making use of these identities in the polynomial term $P_{2}(k)$ of Eq. (105) and in Eq. (111) allow us to separate the finite parts of the $C_{i}$ from their divergent parts proportional to $d_{\epsilon}$ in the following way

$$
\begin{aligned}
& C_{S}=C_{S}^{r}+\frac{3 g_{A}^{2}}{64 \pi^{2} f_{\pi}^{4}} m_{\pi}^{2}\left(2-5 g_{A}^{2}+8 C_{T} f_{\pi}^{2}\right) d_{\epsilon}, \\
& C_{T}=C_{T}^{r}+\frac{3 g_{A}^{2}}{64 \pi^{2} f_{\pi}^{4}} m_{\pi}^{2}\left(1-\frac{5}{2} g_{A}^{2}+8 C_{T} f_{\pi}^{2}\right) d_{\epsilon}, \\
& C_{1}=C_{1}^{r}+\frac{1}{64 \pi^{2} f_{\pi}^{4}}\left(\frac{1}{12}+\frac{5}{6} g_{A}^{2}-\frac{23}{12} g_{A}^{4}\right) d_{\epsilon}, \\
& C_{2}=C_{2}^{r}+\frac{1}{64 \pi^{2} f_{\pi}^{4}}\left(\frac{1}{3}+\frac{10}{3} g_{A}^{2}-\frac{23}{3} g_{A}^{4}\right) d_{\epsilon}, \\
& C_{3}=C_{3}^{r}+\frac{1}{64 \pi^{2} f_{\pi}^{4}}\left(-\frac{3}{2}\right) d_{\epsilon}, \\
& C_{4}=C_{4}^{r}+\frac{1}{64 \pi^{2} f_{\pi}^{4}}\left(\frac{1}{3}+\frac{10}{3} g_{A}^{2}-\frac{23}{3} g_{A}^{4}\right) d_{\epsilon}, \\
& C_{6}=C_{6}^{r}+\frac{1}{64 \pi^{2} f_{\pi}^{4}}\left(\frac{3}{2}\right) d_{\epsilon} .
\end{aligned}
$$

This separation defines also our subtraction scheme, namely all pieces proportional to $d_{\epsilon}$ must be reabsorbed in the LECs multiplying the contact terms. We note that $C_{5}$ and $C_{7}$ have no divergent parts (at order $Q^{2}$ ). With this definition all divergences cancel out and we are left with

$$
\begin{aligned}
v^{(2)}(\mathrm{a} 2+\cdots+\mathrm{a} 7)= & \frac{7 g_{A}^{3} C_{T} m_{\pi}^{2}}{8 \pi^{2} f_{\pi}^{2}}+\frac{7 g_{A}^{2} C_{T} m_{\pi}^{2}}{8 \pi^{2} f_{\pi}^{2}} \boldsymbol{\sigma}_{1} \cdot \boldsymbol{\sigma}_{2} \\
& +\left[C_{1}^{r}+\frac{1}{288 \pi^{2} f_{\pi}^{2}}\left(\frac{5}{8}+\frac{13}{4} g_{A}^{2}-\frac{3}{8} g_{A}^{4}\right)\right] k^{2} \\
& +\left[C_{2}^{r}+\frac{1}{72 \pi^{2} f_{\pi}^{2}}\left(\frac{5}{8}+\frac{13}{4} g_{A}^{2}-\frac{3}{8} g_{A}^{4}\right)\right] K^{2} \\
& +\left[C_{3}^{r}-\frac{g_{A}^{4}}{128 \pi^{2} f_{\pi}^{4}}\right] k^{2} \boldsymbol{\sigma}_{1} \cdot \boldsymbol{\sigma}_{2} \\
& +\left[C_{4}^{r}+\frac{1}{72 \pi^{2} f_{\pi}^{2}}\left(\frac{5}{8}+\frac{13}{4} g_{A}^{2}-\frac{3}{8} g_{A}^{4}\right)\right] K^{2} \boldsymbol{\sigma}_{1} \cdot \boldsymbol{\sigma}_{2} \\
& +i C_{5}^{r} \frac{\boldsymbol{\sigma}_{1}+\boldsymbol{\sigma}_{2}}{2} \cdot \mathbf{K} \times \mathbf{k}+\left[C_{6}^{r}-\frac{g_{A}^{4}}{128 \pi^{2} f_{\pi}^{4}}\right] \boldsymbol{\sigma}_{1} \cdot \mathbf{k} \boldsymbol{\sigma}_{2} \cdot \mathbf{k} \\
& +C_{7}^{r} \boldsymbol{\sigma}_{1} \cdot \mathbf{K} \boldsymbol{\sigma}_{2} \cdot \mathbf{K}+v_{\mathrm{TPE}}^{(2)} \cdot
\end{aligned}
$$




\subsubsection{ONE-PION EXCHANGE CONTRIBUTIONS}

In this subsection we deal with a technical problem, namely the renormalization of loop corrections to OPE contributions. We first construct the set of relevant counter-terms, and then carry out the renormalization of the nucleon and pion masses, field rescaling factors $Z_{\pi}$ and $Z_{N}$, the ratio $g_{A} / f_{\pi}$, and, lastly, loop corrections to the OPE. We define

$$
\pi_{a}=\sqrt{Z_{\pi}} \pi_{a}^{r}, \quad N=\sqrt{Z_{N}} N^{r}
$$

where $\pi_{a}^{r}$ and $N^{r}$ denote, respectively, the renormalized pion and nucleon fields, and $Z_{\pi}$ and $Z_{N}$ are the corresponding field rescaling constants, assumed to have the following expansions

$$
\begin{array}{ll}
Z_{\pi}=1+\delta Z_{\pi}, & \delta Z_{\pi} \sim Q^{2}, \\
Z_{N}=1+\delta Z_{N}, & \delta Z_{N} \sim Q^{2} .
\end{array}
$$

We also define the physical pion mass $m_{\pi}^{r}$ and nucleon mass $m^{r}$ as

$$
\begin{aligned}
m_{\pi}^{r 2} & =m_{\pi}^{2}+\delta m_{\pi}^{2}, \quad \delta m_{\pi}^{2} \sim Q^{4}, \\
m^{r} & =m+\delta m, \quad \delta m \sim Q^{2} .
\end{aligned}
$$

As illustrated in Appendix $\mathrm{C}$, the total Lagrangian, taking the external axial field $A_{a}^{\mu}=0$ in Eq. (440), can be written as

$$
\begin{aligned}
\mathcal{L}= & \bar{N}^{r}\left(i \not \partial-m^{r}+\Gamma_{a}^{0 \prime} \partial_{0} \pi_{a}^{r}+\Lambda_{a}^{i \prime} \partial_{i} \pi_{a}^{r}+\Delta^{\prime}\right) N^{r} \\
& +\frac{1}{2}\left(\partial^{0} \pi_{a}^{r} G_{a b}^{\prime} \partial_{0} \pi_{b}^{r}+\partial^{i} \pi_{a}^{r} \widetilde{G}_{a b}^{\prime} \partial_{i} \pi_{b}^{r}-m_{\pi}^{r 2} \pi_{a}^{r} H_{a b}^{\prime} \pi_{b}^{r}\right) \\
& +\delta m \bar{N}^{r} N^{r}+\delta Z_{N} \bar{N}^{r}\left(i \gamma^{\mu} \partial_{\mu}-m^{r}\right) N^{r}+\frac{\delta m_{\pi}^{2}}{2} \pi_{a}^{r} \pi_{a}^{r},
\end{aligned}
$$

which is expressed in terms of renormalized fields and masses, but bare LECs including $g_{A}$ and $f_{\pi}$. This Lagrangian has essentially the same form as the bare one in Eq. (68) (the primed quantities are defined in Appendix $\mathrm{C}$ ), and leads to an interaction Hamiltonian similar to Eq. (75),

$$
\begin{aligned}
\mathcal{H}_{I}= & \mathcal{H}_{I}[\text { Eq. (75) with primed quantities and renormalized fields and masses ] } \\
& -\delta m \bar{N}^{r} N^{r}-\delta Z_{N} \bar{N}^{r}\left(i \gamma^{i} \partial_{i}-m^{r}\right) N^{r}-\frac{\delta m_{\pi}^{2}}{2} \pi_{a}^{r} \pi_{a}^{r}
\end{aligned}
$$

In addition to the vertices listed in Appendix B, this Hamiltonian generates vertices corresponding to the set of counter-terms in Eqs. (447)-(453), explicit expressions for which follow from those in Appendices B and E. 
The determination of the scaling factors $Z_{\pi}=1+\delta Z_{\pi}$ and $Z_{N}=1+\delta Z_{N}$ for the pion and nucleon fields, the renormalization of the pion and nucleon masses, and $g_{A} / f_{\pi}$ are discussed in detail in Appendices D. The treatment closely follows the steps outlined in Ref. [47]. We only quote the results here:

$$
\begin{aligned}
\delta m_{\pi}^{2} & =2 l_{3} \frac{m_{\pi}^{r 4}}{f_{\pi}^{2}}+\frac{m_{\pi}^{r 2}}{4 f_{\pi}^{2}} J_{01}, \quad \delta Z_{\pi}=-2 \frac{m_{\pi}^{r 2}}{f_{\pi}^{2}} l_{4}+\frac{10 \alpha-1}{2 f_{\pi}^{2}} J_{01}, \\
\delta m & =-4 m_{\pi}^{r 2} c_{1}-\frac{3 g_{A}^{2}}{8 f_{\pi}^{2}} J_{12}, \quad \delta Z_{N}=-\frac{3 g_{A}^{2}}{8 f_{\pi}^{2}} J_{13}, \\
\frac{g_{A}}{f_{\pi}} & =\frac{g_{A}^{r}}{f_{\pi}^{r}}\left(1-\frac{4 m_{\pi}^{r 2}}{g_{A}^{r}} d_{16}+\frac{g_{A}^{r 2}}{3 f_{\pi}^{r 2}} J_{13}+\frac{m \pi^{r 2} l_{4}}{f_{\pi}^{r 2}}\right)\left(1-\frac{2 m_{\pi}^{r 2}}{g_{A}^{r}} d_{18}\right),
\end{aligned}
$$

where the constants $J_{m n}$ are defined in Eq. (392), and $l_{3}$ and $l_{4}\left(c_{1}\right.$ and $\left.d_{i}\right)$ are LECs entering in the subleading $\mathcal{L}_{\pi \pi}^{(4)}\left(\mathcal{L}_{\pi N}^{(2)}\right.$ and $\left.\mathcal{L}_{\pi N}^{(3)}\right)$ chiral Lagrangians. Only leading $Q^{2}$ corrections are provided above, but for $\delta m$ which also includes the sub-leading term of order $Q^{3}$ proportional to $J_{12}$.

We now turn to a discussion of the loop corrections to OPE, illustrated in Fig. 2. In diagrams b2, b4, b6, b8, b11, and b14, the solid dot represents the interaction $-\delta m-4 m_{\pi}^{r 2} c_{1}$, where $\delta m$ is the nucleon mass counter-term.

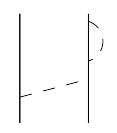

b1

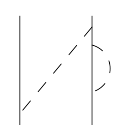

b9

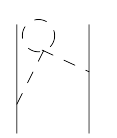

b15

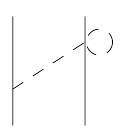

b21

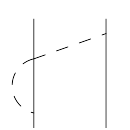

b27

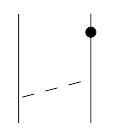

b2

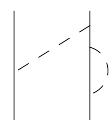

b10

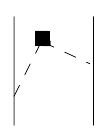

b16

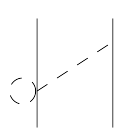

b22

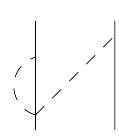

b28

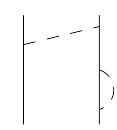

b3

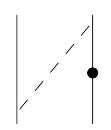

b11

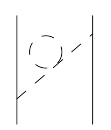

b17

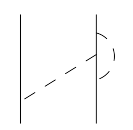

b23

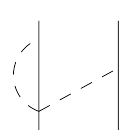

b29

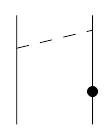

b4

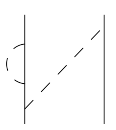

b12

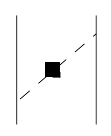

b18
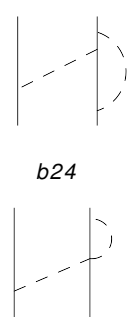

b30

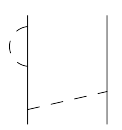

b5

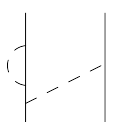

b13

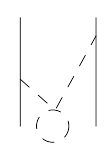

b19
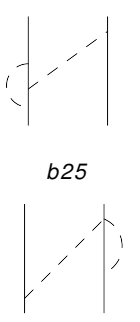

b31

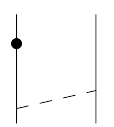

b6

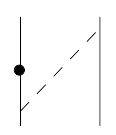

b14

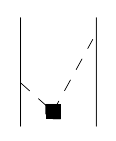

b20
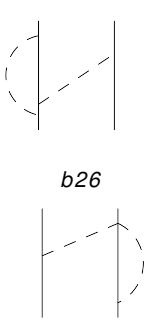

b32

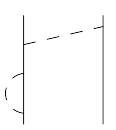

b7

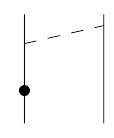

b8

FIG. 2: Half of the possible time-ordered corrections to the OPE potential at order $Q$. Nucleons, and pions are denoted by solid and dashed lines, respectively. See text for further explanations. 
The contributions associated with diagrams b1-b2, b3-b4, b5-b6, b7-b8, b9-b11, and b12b14 represent the renormalization of nucleon external lines and, with the choice of $\delta m$ in Eq. (131), they are seen to vanish. Next, the solid square in diagrams b16, b18, and b20 represents the interaction

$$
\begin{aligned}
H_{2 \pi}^{(4) \prime}= & -\int \mathrm{d} \mathbf{x}\left(\frac{m_{\pi}^{r 2} l_{4}}{f_{\pi}^{2}}+\frac{\delta Z_{\pi}}{2}\right)\left(\boldsymbol{\Pi}^{r} \cdot \boldsymbol{\Pi}^{r}+\partial^{i} \boldsymbol{\pi}^{r} \cdot \partial_{i} \boldsymbol{\pi}^{r}\right) \\
& +\int \mathrm{d} \mathbf{x}\left[\frac{m_{\pi}^{r 4}\left(l_{3}+l_{4}\right)}{f_{\pi}^{2}}+\frac{m_{\pi}^{r 2}}{2} \delta Z_{\pi}-\frac{\delta m_{\pi}^{2}}{2}\right] \boldsymbol{\pi}^{r} \cdot \boldsymbol{\pi}^{r}
\end{aligned}
$$

with vertex (in the convention of Appendix B)

$$
\begin{aligned}
\left\langle 0\left|H_{2 \pi}^{(4) \prime}\right| \mathbf{k}_{1}, a_{1} ; \mathbf{k}_{2}, a_{2}\right\rangle= & \delta_{a_{1}, a_{2}}\left[\left(\frac{2 m_{\pi}^{r 2} l_{4}}{f_{\pi}^{2}}+\delta Z_{\pi}\right)\left(\omega_{1} \omega_{2}-\mathbf{k}_{1} \cdot \mathbf{k}_{2}\right)\right. \\
& \left.+\frac{2 m_{\pi}^{r 4}\left(l_{3}+l_{4}\right)}{f_{\pi}^{2}}+m_{\pi}^{r 2} \delta Z_{\pi}-\delta m_{\pi}^{2}\right] .
\end{aligned}
$$

With $\delta Z_{\pi}$ and $\delta m_{\pi}^{2}$ as given in Eq. (131), the contributions of diagrams b15-b20 cancel out.

Lastly the contributions of diagrams b27-b32 vanish identically, while those in diagrams b21-b26 and b33 sum up to

$$
\begin{aligned}
v^{(2)}(\mathrm{b} 21+\cdots+\mathrm{b} 26+\mathrm{b} 33)= & -\frac{g_{A}^{2}}{4 f_{\pi}^{2}} \boldsymbol{\tau}_{1} \cdot \boldsymbol{\tau}_{2} \frac{\boldsymbol{\sigma}_{1} \cdot \mathbf{k} \boldsymbol{\sigma}_{2} \cdot \mathbf{k}}{\omega_{k}^{2}} \\
& \times\left[8 \frac{m_{\pi}^{2}}{g_{A}} d_{16}-\frac{4 m_{\pi}^{2}}{g_{A}} d_{18}-2 l_{4} \frac{m_{\pi}^{2}}{f_{\pi}^{2}}-\frac{2}{3} \frac{g_{A}^{2}}{f_{\pi}^{2}} J_{13}\right] \\
= & -\frac{g_{A}^{r 2}}{4 f_{\pi}^{r 2}} \boldsymbol{\tau}_{1} \cdot \boldsymbol{\tau}_{2} \frac{\boldsymbol{\sigma}_{1} \cdot \mathbf{k} \boldsymbol{\sigma}_{2} \cdot \mathbf{k}}{\omega_{k}^{2}} \\
& \times\left[8 \frac{m_{\pi}^{r 2}}{g_{A}^{r}} d_{16}-\frac{4 m_{\pi}^{r 2}}{g_{A}^{r}} d_{18}-2 l_{4} \frac{m_{\pi}^{r 2}}{f_{\pi}^{r 2}}-\frac{2}{3} \frac{g_{A}^{r 2}}{f_{\pi}^{r 2}} J_{13}\right],
\end{aligned}
$$

where diagram b33 contains a vertex coming from $H_{\pi N N}^{(3) \prime}$ in Eq. (474), and in the second line the bare couplings and masses have been replaced by the corresponding renormalized quantities, which is correct to order $Q^{2}$. In order to complete the renormalization program to this order, we reconsider diagram a1 in Fig. 1

$$
\begin{aligned}
v(\mathrm{a} 1)= & -\frac{g_{A}^{2}}{4 f_{\pi}^{2}} \boldsymbol{\tau}_{1} \cdot \boldsymbol{\tau}_{2} \frac{\boldsymbol{\sigma}_{1} \cdot \mathbf{k} \boldsymbol{\sigma}_{2} \cdot \mathbf{k}}{\omega_{k}^{2}} \\
= & -\frac{g_{A}^{r 2}}{4 f_{\pi}^{r 2}} \boldsymbol{\tau}_{1} \cdot \boldsymbol{\tau}_{2} \frac{\boldsymbol{\sigma}_{1} \cdot \mathbf{k} \boldsymbol{\sigma}_{2} \cdot \mathbf{k}}{\omega_{k}^{2}} \\
& \times\left[1-8 \frac{m_{\pi}^{r 2}}{g_{A}^{r}} d_{16}+2 \frac{m_{\pi}^{r 2}}{f_{\pi}^{r 2}} l_{4}+\frac{2}{3} \frac{g_{A}^{r 2}}{f_{\pi}^{r 2}} J_{13}\right],
\end{aligned}
$$


where the bare ratio $g_{A} / f_{\pi}$ has been replaced by its renormalized expression (see Ref. [26] for the explicit expression). The renormalized OPE potential up to $Q^{2}$ is then given by the sum of a1 and b21+ $\cdots+\mathrm{b} 26+\mathrm{b} 33$ and reads

$$
v_{\mathrm{OPE}}^{(2)}=-\frac{g_{A}^{r 2}}{4 f_{\pi}^{r 2}} \boldsymbol{\tau}_{1} \cdot \boldsymbol{\tau}_{2}\left(1-\frac{4 m_{\pi}^{r 2}}{g_{A}^{r}} d_{18}\right),
$$

where the factor $\left(1-4 m_{\pi}^{r 2} d_{18} / g_{A}^{r}\right)$ is known as the Goldberger-Treiman discrepancy.

\subsection{NONSTATIC CORRECTIONS TO THE POTENTIAL}

We discuss in this section nonstatic corrections to the potential resulting from expanding energy denominators. These corrections originate from next-to-leading $\left(Q^{0}\right)$ and next-tonext-to-leading $(Q)$ terms in the expansion of the denominators of the various reducible and irreducible topologies, Eq. (82). We will first describe the nonstatic corrections to the OPE potential, and then those to the box topology (diagram a5 of Fig. 1). As it can be seen from Eq. (96) in Ch. 3 these corrections are needed in order to construct the nuclear axial currents up to order $Q$.

\subsubsection{ONE-PION EXCHANGE}

In this subsection we consider non-static corrections to the OPE potential resulting from diagram a1 of Fig. 1. These corrections enter in principle at order $Q$, by keeping the nextto-leading $\left(Q^{0}\right)$ term in the expansion of the pionic energy denominator, and lead to

$$
v^{(1)}(\mathrm{a} 1)=v^{(0)}(\mathrm{a} 1) \frac{\left(E_{1}^{\prime}+E_{2}^{\prime}-E_{1}-E_{2}\right)}{\omega_{k}} .
$$

However this correction vanishes on the energy shell $E_{i}=E_{f}$ with $E_{i}=E_{1}+E_{2}$ and $E_{f}=E_{1}^{\prime}+E_{2}^{\prime}$ where $E_{i}\left(\mathbf{p}_{i}\right)$ are the initial and final energies (momenta) of nucleon $i$. We take $v^{(1)}$ to also vanish off-the-energy-shell.

At order $Q^{2}$ the nonstatic correction to the OPE can be written as

$$
v^{(2)}(\mathrm{a} 1)=v_{\mathrm{OPE}}^{(0)} \frac{\left(E_{1}^{\prime}-E_{1}\right)^{2}+\left(E_{2}^{\prime}-E_{2}\right)^{2}}{2 \omega_{k}^{2}},
$$

or equivalently on the energy shell as

$$
v^{(2)}(\mathrm{a} 1)=-v_{\mathrm{OPE}}^{(0)} \frac{\left(E_{1}^{\prime}-E_{1}\right)\left(E_{2}^{\prime}-E_{2}\right)}{\omega_{k}^{2}} .
$$

These two forms differ off-the-energy-shell (i.e., $E_{1}^{\prime}+E_{2}^{\prime} \neq E_{1}+E_{2}$ ), and this fact turns out to be relevant in the calculation of nonstatic corrections to the box diagram at order $Q^{3}$. It 
is convenient to parameterize these corrections in terms of a parameter $\nu$ (as was first done in Ref. [43])

$$
v_{\mathrm{OPE}, \mathrm{ns}}^{(2)}(\nu)=v_{\mathrm{OPE}}^{(0)} \frac{(1-\nu)\left[\left(E_{1}^{\prime}-E_{1}\right)^{2}+\left(E_{2}^{\prime}-E_{2}\right)^{2}\right]-2 \nu\left(E_{1}^{\prime}-E_{1}\right)\left(E_{2}^{\prime}-E_{2}\right)}{2 \omega_{k}^{2}},
$$

where $\mathbf{k}=\mathbf{p}_{1}^{\prime}-\mathbf{p}_{1}$ is the momentum transfer. Note that Eqs. (140) and (141) are obtained for $\nu=0$ and $\nu=1$, respectively. Thus, there is an infinite class of corrections $v_{\mathrm{OPE}, \mathrm{ns}}^{(2)}(\nu)$, labeled by the parameter $\nu$, which, while equivalent on the energy shell and hence independent of $\nu$, are different off-the-energy-shell. Friar [43] has in fact shown that these different off-theenergy-shell extrapolations $v_{\mathrm{OPE}, \mathrm{ns}}^{(2)}(\nu)$ are unitarily equivalent (see also Ref. [23]).

\subsubsection{TWO-PION EXCHANGE AT ORDER $Q^{3}$}

For the derivation of nuclear axial currents knowledge of the two-nucleon potential up to order $Q^{3}$ is required. This correction results from

$$
\begin{aligned}
v^{(3)}=T^{(3)} & -\left[v^{(0)} G_{0} v^{(0)} G_{0} v^{(0)} G_{0} v^{(0)}\right] \\
& -\left[v^{(2)} G_{0} v^{(0)}+v^{(0)} G_{0} v^{(2)}\right],
\end{aligned}
$$

where we have made use of the fact that $v^{(1)}=0$. The calculation of the full $v^{(3)}$ contribution is rather involved, see Ref. [23]. In the following we report only the expressions obtained for the two topologies needed to calculate the axial currents up to order $Q$ : diagrams a3 and a5 of Fig. 1. Both diagrams, at order $Q^{3}$, have contributions from (i) vertex corrections (coming from $\mathcal{L}_{\pi N}^{(2)}$, and of no interest here), and (ii) nonstatic corrections resulting from the expansion of the pionic energy denominators (considered here).
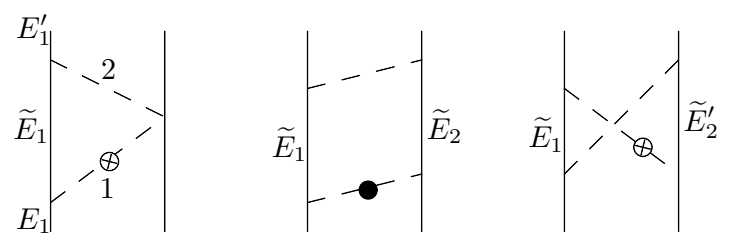

FIG. 3: Some of the diagrams illustrating the nonstatic corrections to diagram a3 and a5 of Fig. (1) at order $Q^{3}$. Pion lines with crossed (full) circle indicate that only the nextto-leading $Q^{0}$ (next-to-next-to-leading $Q$ ) term in the expansion of energy denominators, Eq. (82), are retained in the corresponding amplitudes. Only one among the possible time orderings is shown. 
The calculation of diagram a3 is straightforward [23], and gives

$$
v_{\mathrm{TPE}, \triangle}^{(3)}=-\frac{g_{A}^{2}}{16 f_{\pi}^{4}} \boldsymbol{\tau}_{1} \cdot \boldsymbol{\tau}_{2} \int \frac{d \mathbf{q}_{1}}{(2 \pi)^{3}} \frac{1}{\omega_{1}^{2} \omega_{2}^{2}}\left(\mathbf{q}_{1} \cdot \mathbf{q}_{2}+i \boldsymbol{\sigma}_{1} \cdot \mathbf{q}_{2} \times \mathbf{q}_{1}\right)\left(E_{1}+E_{1}^{\prime}-2 \widetilde{E}_{1}\right),
$$

where $\omega_{1}=\sqrt{\mathbf{q}_{1}^{2}+m_{\pi}^{2}}$ (and similarly for $\omega_{2}$ ), $\widetilde{E}_{1}$ is the intermediate energy as showed in Fig. 3, and $\mathbf{q}_{2}=\mathbf{k}-\mathbf{q}_{1}$. The contribution of diagram a5 depends on the off-the-energy-shell prescription adopted for $v_{\mathrm{OPE}, \mathrm{ns}}^{(2)}(\nu)$ albeit different prescriptions lead to unitarily equivalent corrections to $v_{\mathrm{TPE}}^{(3)}(\nu)[23]$. Here we report the correction corresponding to $\nu=0$, i.e.

$$
\begin{aligned}
v_{\mathrm{TPE}, \square}^{(3)}(\nu=0) & =-\frac{g_{A}^{4}}{32 f_{\pi}^{4}}\left(3+2 \boldsymbol{\tau}_{1} \cdot \boldsymbol{\tau}_{2}\right) \int \frac{d \mathbf{q}_{1}}{(2 \pi)^{3}}\left(\boldsymbol{\sigma}_{1} \cdot \mathbf{q}_{2}\right)\left(\boldsymbol{\sigma}_{1} \cdot \mathbf{q}_{1}\right)\left(\boldsymbol{\sigma}_{2} \cdot \mathbf{q}_{1}\right)\left(\boldsymbol{\sigma}_{2} \cdot \mathbf{q}_{2}\right) \\
& \times\left(\frac{E_{1}-\widetilde{E}_{1}+E_{2}^{\prime}-\widetilde{E}_{2}^{\prime}}{\omega_{1}^{4} \omega_{2}^{2}}+\frac{E_{1}^{\prime}-\widetilde{E}_{1}+E_{2}-\widetilde{E}_{2}^{\prime}}{\omega_{1}^{2} \omega_{2}^{4}}\right),
\end{aligned}
$$

where $\widetilde{E}_{1}, \widetilde{E}_{2}$, and $\widetilde{E}_{2}^{\prime}$ are the intermediate nucleonic energies as shown in Fig. 3. 


\section{CHAPTER 5}

\section{NUCLEAR AXIAL CURRENTS UP TO ONE LOOP}

In this chapter we discuss the derivation of the nuclear axial charge and current operators in chiral effective field theory up to one loop. The derivation is based on TOPT and accounts for cancellations between the contributions of irreducible diagrams and the contributions from nonstatic corrections of energy denominators in reducible diagrams. Ultraviolet divergencies associated with the loop corrections are isolated in dimensional regularization. The resulting axial current is finite and conserved in the chiral limit, while the axial charge requires normalization. A complete set of contact terms for the axial charge up to the relevant order in the power counting is constructed.

Hereafter, the momenta $\mathbf{k}_{i}$ and $\mathbf{K}_{i}$ are defined as

$$
\mathbf{K}_{i}=\left(\mathbf{p}_{i}^{\prime}+\mathbf{p}_{i}\right) / 2, \quad \mathbf{k}_{i}=\mathbf{p}_{i}^{\prime}-\mathbf{p}_{i},
$$

where $\mathbf{p}_{i}\left(\mathbf{p}_{i}^{\prime}\right)$ is the initial (final) momentum of nucleon $i$. A symmetrization $(1 \rightleftharpoons 2)$ and an overall momentum-conserving $\delta$-function $(2 \pi)^{3} \delta\left(\mathbf{k}_{1}+\mathbf{k}_{2}-\mathbf{q}\right)$ are understood in all terms listed below unless otherwise noted. While the potential in Ch. 4 has been derived in the center-of-mass frame, the currents derived here are in a generic frame.

There is an earlier but incomplete derivation by Rho and collaborators $[28,48]$ of weak axial currents in $\chi \mathrm{EFT}$, based on heavy-baryon perturbation theory. There is also a recent derivation by the Bochum-Bonn group, based on TOPT and the unitary transformation method, which has appeared a few months after our own work [49]. A comparison between the various derivations is presented at the end of this chapter.

\subsection{ONE-BODY AXIAL CHARGE AND CURRENT}

We begin by discussing how the leading-order one-body operators are generated by the chiral Hamiltonians. The relevant diagrams are shown in Fig. 4. The contribution of panels a1 and a2 lead to the single-nucleon current given by

$$
\mathbf{j}_{5, a}^{(-3)}(\mathbf{q})=-\frac{g_{A}}{2} \tau_{1, a}\left[\boldsymbol{\sigma}_{1}-\frac{\mathbf{q}}{q^{2}+m_{\pi}^{2}} \boldsymbol{\sigma}_{1} \cdot \mathbf{q}\right](2 \pi)^{3} \delta\left(\mathbf{k}_{1}-\mathbf{q}\right),
$$


while the contribution of panel a3 to the single-nucleon axial charge

$$
\rho_{5, a}^{(-2)}(\mathbf{q})=-\frac{g_{A}}{2 m} \tau_{1, a} \boldsymbol{\sigma}_{1} \cdot\left(\mathbf{K}_{1}\right)(2 \pi)^{3} \delta\left(\mathbf{k}_{1}-\mathbf{q}\right) .
$$

Here $\mathbf{q}$ is the momentum carried by the external field and we made explicit the momentum conserving $\delta$-function. The counting $Q^{-3}$ of $\mathbf{j}_{5, a}$ (panel a1 in Fig. 4) follows from the product of a factor $Q^{0}$ associated with the $N N A$ vertex (the external field has been removed), and a factor $Q^{-3}$ due to the momentum-conserving $\delta$-function $\delta\left(\mathbf{p}_{2}^{\prime}-\mathbf{p}_{2}\right)$ implicit in disconnected terms of this type. Evaluation of the pion-pole contribution (panel a3), in which the axial source couples directly to the pion which is then absorbed by the nucleon, leads to the $\rho_{5, a}^{(-2)}$ expression in Eq. (148). In this disconnected term, the counting $Q^{-2}$ accounts for the $Q^{-3}$ factor due to $\delta\left(\mathbf{p}_{2}^{\prime}-\mathbf{p}_{2}\right)$, the factors $Q$ and $Q^{2}$ of the $\pi A$ and $\pi N N$ vertices, respectively, and the factor $Q^{-2}$ from the pion field normalization and energy denominator associated with the intermediate state. A similar counting is applied to panel a2 in Fig. 4 contributing to $\mathbf{j}_{5, a}$.

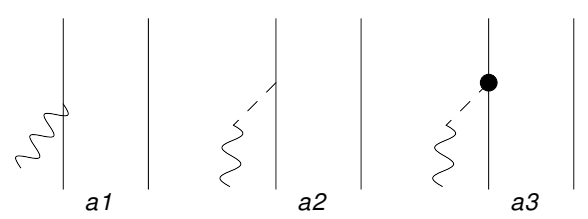

FIG. 4: Diagrams a1 and a2 contribute to the one-body axial current operator at order $Q^{(-3)}$. Diagram a3 contributes to the one-body axial charge operator at order $Q^{(-2)}$. Nucleons, pions, and axial fields are denoted by solid, dashed, and wavy lines, respectively. Only a single time ordering is shown for diagrams a2 and a3. The full dot in $\mathrm{c}$ is from the interaction vertex $H_{\pi N N}^{(2)}$, see Appendix B.

There is no direct coupling of the nucleon to the time-component $A_{a}^{0}$ of the axial field: the interaction $-\left(g_{A} / 2\right) \bar{N} \boldsymbol{\tau} \cdot \mathbf{A}_{0} \gamma^{0} \gamma^{5} N$ in the term

$$
-\bar{N} \Delta(2) N
$$

with $\Delta(2)$ as given by Eq. (384) occurs with the opposite sign in the term

$$
-\left(f_{\pi} / 2\right)\left[\bar{N} \Gamma_{a}^{0}(1) N\left(G^{-1}\right)_{a b} F_{b c} A_{c}^{0}+\text { h.c. }\right],
$$

with $\Gamma_{a}^{0}(1)$ as in Eq. (382) with $(G)_{a b}^{-1}=F_{a b}=\delta_{a b}$ up to $\pi_{a} \pi_{b}$ or $m_{\pi}^{2}$ terms, and hence cancels out in the Hamiltonian of Eq. (75). The single-nucleon axial charge of the correct sign and 
strength follows from the sum of the two time-ordered contributions of diagram a3 with the full dot representing the interaction $\left(g_{A} / 2 f_{\pi}\right) \bar{N} \boldsymbol{\tau} \cdot \Pi \gamma^{0} \gamma^{5} N$ from

$$
-(1 / 2)\left[\Pi_{a}\left(G^{-1}\right)_{a b} \bar{N} \Gamma_{b}^{0}(1) N+\text { h.c. }\right] .
$$

There are relativistic corrections suppressed by two powers of $Q$ to these leading order operators. We only consider those to the single-nucleon axial current, since they have been found to give significant contributions to the cross section for proton weak capture on ${ }^{3} \mathrm{He}$ of interest in solar physics [48],

$$
\begin{aligned}
& \mathbf{j}_{5, a}^{(-1)}(\mathrm{a} 4)=\frac{g_{A}}{4 m^{2}} \tau_{1, a}\left[K_{1}^{2} \boldsymbol{\sigma}_{1}+\frac{i}{2} \mathbf{k}_{1} \times \mathbf{K}_{1}-\boldsymbol{\sigma}_{1} \cdot \mathbf{K}_{1} \mathbf{K}_{1}+\frac{1}{4} \boldsymbol{\sigma}_{1} \cdot \mathbf{k}_{1} \mathbf{k}_{1}\right], \\
& \mathbf{j}_{5, a}^{(-1)}(\mathrm{a} 5)=-\frac{\mathbf{q}}{q^{2}+m_{\pi}^{2}}\left[\mathbf{q} \cdot \mathbf{j}_{5, a}^{(-1)}(\mathrm{a} 4)+\frac{g_{A}}{2 m^{2}} \tau_{1, a} \boldsymbol{\sigma}_{1} \cdot \mathbf{K}_{1} \mathbf{k}_{1} \cdot \mathbf{K}_{1}\right] .
\end{aligned}
$$

Diagram a5 contains two contributions at order $Q^{-1}$ : one is from the $1 / \mathrm{m}^{2}$ terms originating from the non-relativistic expansion of the $\pi N N$ interaction $H_{\pi N N}^{(1)}$; the other is due to the $1 / m$ terms in $H_{\pi N N}^{(2)}$ and the (leading) non-static corrections (proportional to $1 / m$ ) to energy denominators.

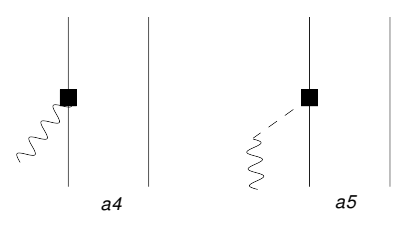

FIG. 5: Diagrams illustrating the relativistic corrections to the one-body axial current. Nucleons, pions, and axial fields are denoted by solid, dashed, and wavy lines, respectively. Only a single time ordering is shown for diagram a5. See text for further explanations.

Loop corrections to the one-body axial charge and current, relevant for the renormalization of these operators, are derived in TOPT in Appendix F. They are in agreement with the results obtained in heavy-baryon perturbation theory [50].

\subsection{TWO-BODY AXIAL CHARGE}

The weak axial charge two-body operator, in analogy with the two-nucleon potential, can be written as

$$
\rho_{5, a}=\rho_{5, a}(\mathrm{OPE})+\rho_{5, a}(\mathrm{TPE})+\rho_{5, a}(\mathrm{CT})
$$


namely as a sum of terms due to one-pion exchange (OPE), two-pion exchange (TPE), and contact contributions (CT). In the following, and in Sec. 5.3 as well, contributions to the OPE and TPE (or MPE in Sec. 5.3) operators are labeled by the power counting superscript $(n)$. While each individual contribution is not explicitly identified as being OPE or TPE (or $\mathrm{MPE}$ ), this is obvious from the context.

\subsubsection{ONE- AND TWO-PION EXCHANGE CONTRIBUTIONS}

Diagrams contributing to $\rho_{5, a}^{\mathrm{OPE}}$ at leading order are shown in Fig. 6. Tree level diagrams in panels b1 and b2 of this figure enter at order $Q^{-1}$ and read

$$
\begin{aligned}
\rho_{5, a}^{(-1)}(\mathrm{b} 1) & =i \frac{g_{A}}{8 f_{\pi}^{2}}\left(\boldsymbol{\tau}_{1} \times \boldsymbol{\tau}_{2}\right)_{a} \boldsymbol{\sigma}_{2} \cdot \mathbf{k}_{2} \frac{1}{\omega_{2}^{2}}, \\
\rho_{5, a}^{(-1)}(\mathrm{b} 2) & =\rho_{5, a}^{(-1)}(\mathrm{b} 1) .
\end{aligned}
$$

We note that the sum b1+b2 gives the leading order OPE axial charge operator first derived in Ref. [51] using soft-pion theorem.
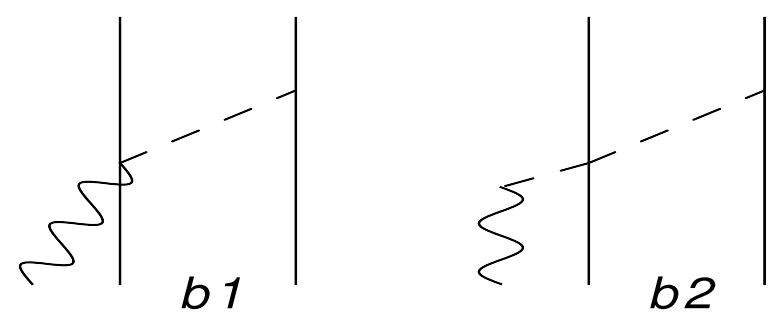

FIG. 6: Diagrams contributing to the OPE axial charge at leading order $Q^{-1}$ (panels b1 and b2). Nucleons, pions, and axial fields are denoted by solid, dashed, and wavy lines, respectively. Only a single time ordering is shown for each topology.

At order $Q^{0}$ we have in principle nonstatic contributions coming from the expansion of the pionic energy denominators, and contributions coming from vertices generated from chiral Hamiltonians $\mathcal{H}_{\pi N N}^{(2)}$. Both these corrections, displayed in Fig. 7, are seen to vanish when summing over all time orderings (diagrams b3-b6). We note that there are no reducible contributions up to order $Q^{0}$. 


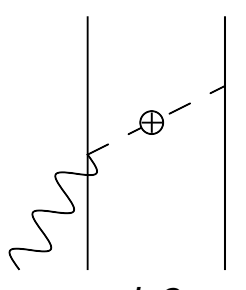

b3

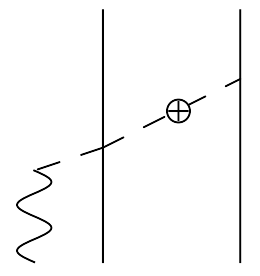

b4

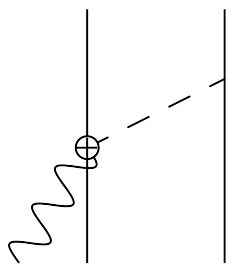

b5

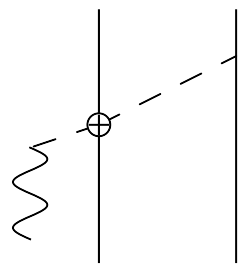

b6

FIG. 7: Diagrams contributing to the OPE axial charge at order $Q^{0}$. Nucleons, pions, and axial fields are denoted by solid, dashed, and wavy lines, respectively. Pion lines (pion lines with crossed circle) indicate that only the leading $Q^{-1}$ (next-to-leading $Q^{0}$ ) term in the expansion of the energy denominators, Eq. (82), are retained in the corresponding amplitudes. Crossed circle vertices indicate terms coming from next-to-leading $Q^{2}$ chiral Hamiltonians. Only a single time ordering is shown for each topology.

Two-pion exchange contributions are shown in panels c1-c12 of Fig. 8. The individual contributions read

$$
\begin{aligned}
\rho_{5, a}^{(1)}(\mathrm{c} 1+\mathrm{c} 2)= & i \frac{g_{A}}{16 f_{\pi}^{4}}\left(\boldsymbol{\tau}_{1} \times \boldsymbol{\tau}_{2}\right)_{a} \boldsymbol{\sigma}_{1} \cdot \mathbf{k}_{2} I^{(0)}\left(k_{2}\right), \\
\rho_{5, a}^{(1)}(\mathrm{c} 5+\mathrm{c} 6)= & i \frac{g_{A}^{3}}{16 f_{\pi}^{4}}\left[4 \tau_{1, a} \sigma_{1 i}\left(\boldsymbol{\sigma}_{2} \times \mathbf{k}_{2}\right)_{j} J_{i j}^{(2)}\left(\mathbf{k}_{2}\right)\right. \\
& \left.+\left(\boldsymbol{\tau}_{1} \times \boldsymbol{\tau}_{2}\right)_{a}\left[k_{2}^{2} J^{(0)}\left(k_{2}\right)-J^{(2)}\left(k_{2}\right)\right] \boldsymbol{\sigma}_{1} \cdot \mathbf{k}_{2}\right],
\end{aligned}
$$

while those of $\mathrm{c} 3-\mathrm{c} 4, \mathrm{c} 7-\mathrm{c} 8$, and $\mathrm{c} 9-\mathrm{c} 12$ vanish, after summing over all time orderings. The freedom in the choice of pion field, parametrized by the parameter $\alpha$ in Appendix A, introduces an $\alpha$-dependence in the interaction vertices with three or four pions, see Appendix B. The contributions of diagrams $\mathrm{c} 4$ and $\mathrm{c} 8$, which include a $3 \pi$ vertex, turn out to vanish identically. But in general this $\alpha$ dependence must cancel out exactly in the calculation of the scattering amplitude, as is indeed the case for the two-nucleon axial charge and current operators obtained in this Thesis. The loop functions have been defined in Eqs. (102)-(104) of the previous chapter, and they have been evaluated in dimensional regularization Ref. [22]. 

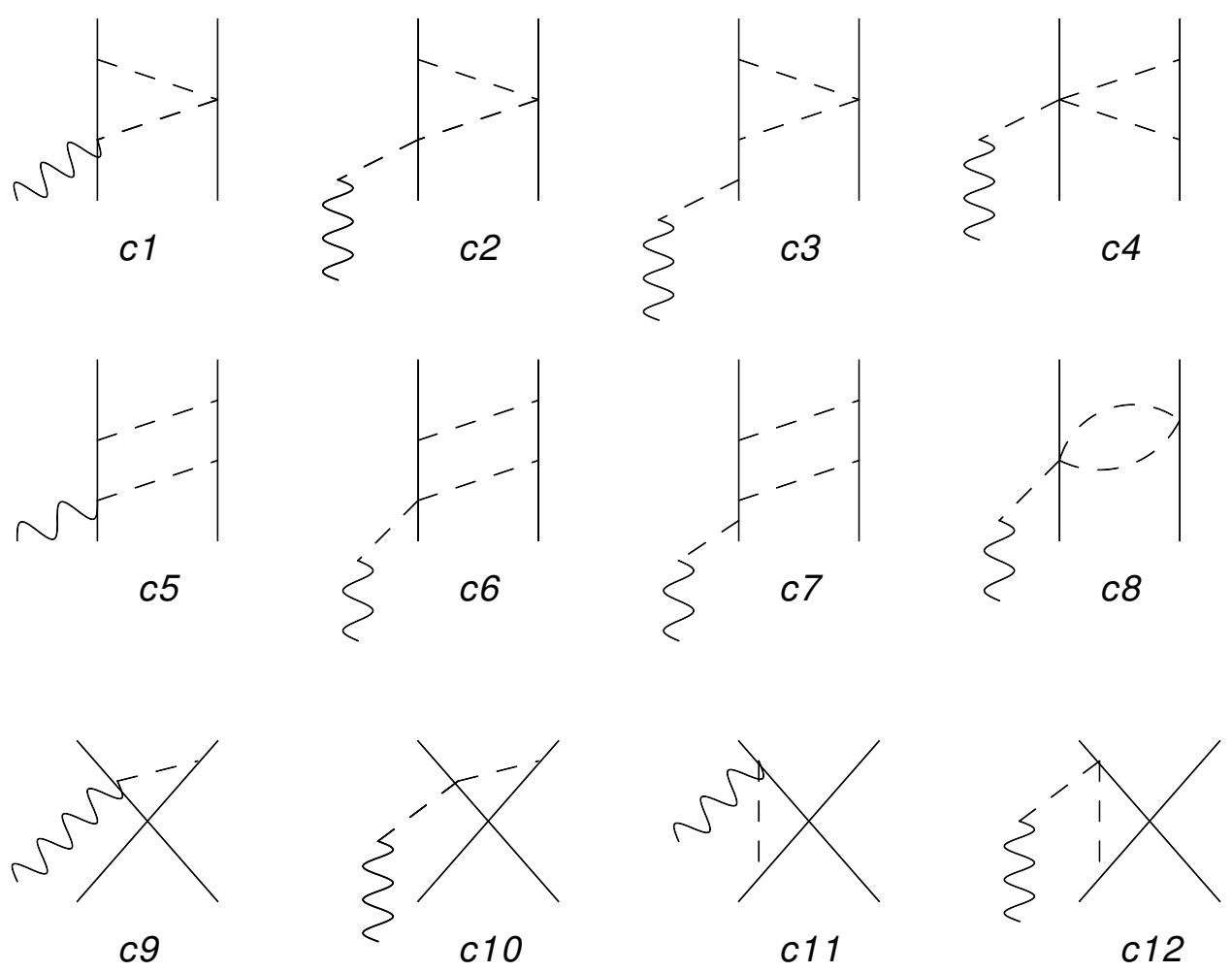

FIG. 8: Diagrams contributing to the OPE axial charge at leading order $Q^{-1}$ (panels a1 and a2), and to the TPE axial charge operator at order $Q$. Nucleons, pions, and axial fields are denoted by solid, dashed, and wavy lines, respectively. Only a single time ordering is shown for each topology.

Insertion of the finite parts of these loop functions leads to

$$
\begin{aligned}
\rho_{5, a}^{(1)}(\mathrm{c} 1+\mathrm{c} 2)= & -i \frac{g_{A}}{128 \pi^{2} f_{\pi}^{4}}\left(\boldsymbol{\tau}_{1} \times \boldsymbol{\tau}_{2}\right)_{a} \boldsymbol{\sigma}_{1} \cdot \mathbf{k}_{2} \frac{s_{2}}{k_{2}} \ln \left(\frac{s_{2}+k_{2}}{s_{2}-k_{2}}\right) \\
\rho_{5, a}^{(1)}(\mathrm{c} 5+\mathrm{c} 6)= & -i \frac{g_{A}^{3}}{128 \pi^{2} f_{\pi}^{4}}\left[4 \tau_{1, a}\left(\boldsymbol{\sigma}_{1} \times \boldsymbol{\sigma}_{2}\right) \cdot \mathbf{k}_{2} \frac{s_{2}}{k_{2}} \ln \frac{s_{2}+k_{2}}{s_{2}-k_{2}}\right. \\
& \left.-\left(\boldsymbol{\tau}_{1} \times \boldsymbol{\tau}_{2}\right)_{a} \boldsymbol{\sigma}_{1} \cdot \mathbf{k}_{2} \frac{k_{2}^{2}+2 s_{2}^{2}}{k_{2} s_{2}} \ln \frac{s_{2}+k_{2}}{s_{2}-k_{2}}\right]
\end{aligned}
$$

where

$$
s_{j}=\sqrt{4 m_{\pi}^{2}+\mathbf{k}_{j}^{2}}
$$


The divergent and logarithm-free parts read

$$
\begin{aligned}
\left.\rho_{5, a}^{(1)}(\mathrm{c} 1+\mathrm{c} 2)\right|_{\infty}= & -i \frac{g_{A}}{128 \pi^{2} f_{\pi}^{4}}\left(\boldsymbol{\tau}_{1} \times \boldsymbol{\tau}_{2}\right)_{a} \boldsymbol{\sigma}_{1} \cdot \mathbf{k}_{2}\left(d_{\epsilon}-1\right) \\
\left.\rho_{5, a}^{(1)}(\mathrm{c} 5+\mathrm{c} 6)\right|_{\infty}= & -i \frac{g_{A}^{3}}{32 \pi^{2} f_{\pi}^{4}}\left[\tau_{1, a}\left(\boldsymbol{\sigma}_{1} \times \boldsymbol{\sigma}_{2}\right) \cdot \mathbf{k}_{2}\left(d_{\epsilon}-\frac{1}{3}\right)\right. \\
& \left.-\frac{3}{4}\left(\boldsymbol{\tau}_{1} \times \boldsymbol{\tau}_{2}\right)_{a} \boldsymbol{\sigma}_{1} \cdot \mathbf{k}_{2}\left(d_{\epsilon}+\frac{1}{3}\right)\right]
\end{aligned}
$$

with the constant $d_{\epsilon}$ defined in Eq. (110).

\subsubsection{CONTACT CONTRIBUTIONS}

At order $Q^{0}$ there are no contact terms contributing to $\rho_{5, a}^{\mathrm{CT}}$, and this fact is proven in Appendix G. Those at order $Q$ are given by (see Appendix $\mathrm{G}$ for a derivation)

$$
\rho_{5, a}(\mathrm{CT})=\sum_{i=1}^{4} z_{i} O_{i}
$$

where the $z_{i}$ are (unknown) LECs and the operators $O_{i}$ with $i=1, \ldots, 4$, symmetrized with respect to the exchange $1 \rightleftharpoons 2$, have been defined as

$$
\begin{aligned}
& O_{1}=i\left(\boldsymbol{\tau}_{1} \times \boldsymbol{\tau}_{2}\right)_{a}\left(\boldsymbol{\sigma}_{1} \cdot \mathbf{k}_{2}-\boldsymbol{\sigma}_{2} \cdot \mathbf{k}_{1}\right), \\
& O_{2}=i\left(\boldsymbol{\tau}_{1} \times \boldsymbol{\tau}_{2}\right)_{a}\left(\boldsymbol{\sigma}_{1} \cdot \mathbf{k}_{1}-\boldsymbol{\sigma}_{2} \cdot \mathbf{k}_{2}\right), \\
& O_{3}=i\left(\boldsymbol{\sigma}_{1} \times \boldsymbol{\sigma}_{2}\right) \cdot\left(\tau_{1, a} \mathbf{k}_{2}-\tau_{2, a} \mathbf{k}_{1}\right), \\
& O_{4}=\left(\tau_{1, a}-\tau_{2, a}\right)\left(\boldsymbol{\sigma}_{1}-\boldsymbol{\sigma}_{2}\right) \cdot\left(\mathbf{K}_{1}+\mathbf{K}_{2}\right) .
\end{aligned}
$$

We observe that the loop divergencies from c1-c2 and c5-c6 can be reabsorbed in the LECs $z_{1}$ and $z_{3}$, in the following way

$$
\begin{aligned}
z_{1} & =z_{1}^{r}+i \frac{g_{A}}{128 \pi^{2} f_{\pi}^{4}}\left(1-3 g_{A}^{2}\right) d_{\epsilon} \\
z_{3} & =z_{3}^{r}+i \frac{g_{A}^{3}}{32 \pi^{2} f_{\pi}^{4}} d_{\epsilon}
\end{aligned}
$$

Therefore in this subtraction scheme

$$
\begin{aligned}
\left.\rho_{5, a}^{(1)}(\mathrm{c} 1+\mathrm{c} 2+\mathrm{c} 5+\mathrm{c} 6)\right|_{\infty}+z_{1} O_{1}+z_{3} O_{3} & =\left[z_{1}^{r}-i \frac{g_{A}}{128 \pi^{2} f_{\pi}^{4}}\left(1-g_{A}^{2}\right)\right] O_{1} \\
& +\left[z_{3}^{r}-i \frac{g_{A}^{3}}{32 \pi^{2} f_{\pi}^{4}}\right] O_{3} .
\end{aligned}
$$




\subsection{TWO-BODY AXIAL CURRENT}

As for the charge, the two-body current is written as a sum of one-pion exchange (OPE), multi-pion exchange (MPE), and contact (CT) terms (notation and conventions are as in Sec. 5.2),

$$
\mathbf{j}_{5, a}=\mathbf{j}_{5, a}(\mathrm{OPE})+\mathbf{j}_{5, a}(\mathrm{MPE})+\mathbf{j}_{5, a}(\mathrm{CT}) .
$$

We discuss $\mathbf{j}_{5, a}^{\mathrm{CT}}$ here. It is well known [52] that a single contact term occurs at order $Q^{0}$ (Fig. 9), and none at order $Q$. This term is taken (in the properly symmetrized form) as

$$
\mathbf{j}_{5, a}(\mathrm{CT})=z_{0}\left[\left(\boldsymbol{\tau}_{1} \times \boldsymbol{\tau}_{2}\right)_{a} \boldsymbol{\sigma}_{1} \times \boldsymbol{\sigma}_{2}-\frac{\mathbf{q}}{q^{2}+m_{\pi}^{2}}\left(\boldsymbol{\tau}_{1} \times \boldsymbol{\tau}_{2}\right)_{a} \mathbf{q} \cdot\left(\boldsymbol{\sigma}_{1} \times \boldsymbol{\sigma}_{2}\right)\right],
$$

where the second term is the pion-pole contribution. This contact term originates from the interaction Lagrangian $\left(\bar{N} \gamma^{\mu} \gamma_{5} u_{\mu} N\right) \bar{N} N$ and the LEC $z_{0}$ is related to the LEC $c_{D}$ (in standard notation) entering the three-nucleon potential at leading order [52]. The LEC $z_{0}$ is fixed by reporducing the Gamow-Teller matrix element contributing to tritium $\beta$-decay and is discussed in the next chapter.
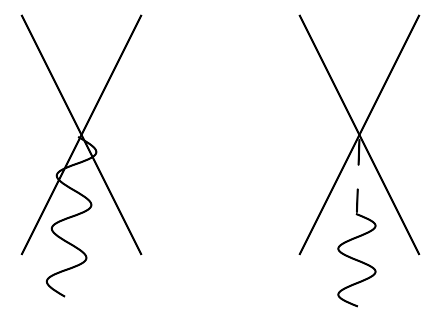

FIG. 9: Contact and corresponding pion-pole term occuring at order $Q^{0}$ in the axial current. Nucleons, pions, and axial fields are denoted by solid, dashed, and wavy lines, respectively. Only a single time ordering is shown for the pion-pole topology (second diagram).

\subsubsection{LEADING ONE- AND MULTI-PION EXCHANGE CONTRIBUTIONS}

Leading contributions to $\mathbf{j}_{5, a}(\mathrm{OPE})$ and $\mathbf{j}_{5, a}(\mathrm{MPE})$ are shown, respectively, in panels d1d2, and panels e1-e25 of Fig. 10. There are no contributions at order $Q^{-1}$ from diagrams d1 and d2: in d1 the interaction $H_{\pi N N A}^{(1)}$ contains no coupling to the field $\mathbf{A}_{a}$, while in d2 the sum over the 6 time orderings, when leading order vertices from $H_{\pi A}^{(2)}, H_{2 \pi N N}^{(1)}$, and $H_{\pi N N}^{(1)}$ are 
considered, vanishes. The first non-vanishing contributions enter at order $Q^{0}$, and read

$$
\begin{aligned}
\mathbf{j}_{5, a}^{(0)}(\mathrm{d} 1)= & \frac{g_{A}}{2 f_{\pi}^{2}}\left(\boldsymbol{\tau}_{1} \times \boldsymbol{\tau}_{2}\right)_{a}\left[i \frac{\mathbf{K}_{1}}{2 m}-\frac{c_{6}+1}{4 m} \boldsymbol{\sigma}_{1} \times \mathbf{q}+\left(c_{4}+\frac{1}{4 m}\right) \boldsymbol{\sigma}_{1} \times \mathbf{k}_{2}\right] \boldsymbol{\sigma}_{2} \cdot \mathbf{k}_{2} \frac{1}{\omega_{2}^{2}} \\
& +\frac{g_{A}}{f_{\pi}^{2}} c_{3} \tau_{2, a} \mathbf{k}_{2} \boldsymbol{\sigma}_{2} \cdot \mathbf{k}_{2} \frac{1}{\omega_{2}^{2}}, \\
\mathbf{j}_{5, a}^{(0)}(\mathrm{d} 2)= & -\frac{g_{A}}{2 f_{\pi}^{2}} \frac{\mathbf{q}}{q^{2}+m_{\pi}^{2}}\left[\tau_{2, a}\left(4 c_{1} m_{\pi}^{2}+2 c_{3} \mathbf{q} \cdot \mathbf{k}_{2}\right)-c_{4}\left(\boldsymbol{\tau}_{1} \times \boldsymbol{\tau}_{2}\right)_{a} \boldsymbol{\sigma}_{1} \cdot\left(\mathbf{q} \times \mathbf{k}_{2}\right)\right] \boldsymbol{\sigma}_{2} \cdot \mathbf{k}_{2} \frac{1}{\omega_{2}^{2}} \\
& -i \frac{g_{A}}{16 m f_{\pi}^{2}} \frac{\mathbf{q}}{q^{2}+m_{\pi}^{2}}\left(\boldsymbol{\tau}_{1} \times \boldsymbol{\tau}_{2}\right)_{a}\left(2 \mathbf{K}_{1}+i \boldsymbol{\sigma}_{1} \times \mathbf{k}_{1}\right) \cdot\left(\mathbf{q}+\mathbf{k}_{2}\right) \boldsymbol{\sigma}_{2} \cdot \mathbf{k}_{2} \frac{1}{\omega_{2}^{2}} \\
& +i \frac{g_{A}}{8 m f_{\pi}^{2}} \frac{\mathbf{q}}{q^{2}+m_{\pi}^{2}}\left(\boldsymbol{\tau}_{1} \times \boldsymbol{\tau}_{2}\right)_{a}\left(\mathbf{K}_{1} \cdot \mathbf{k}_{1}+2 \mathbf{K}_{2} \cdot \mathbf{k}_{2}\right) \boldsymbol{\sigma}_{2} \cdot \mathbf{k}_{2} \frac{1}{\omega_{2}^{2}} .
\end{aligned}
$$

For the diagrams contributing to $\mathbf{j}_{5, a}(\mathrm{MPE})$ only a single time ordering is displayed for each topology. It is understood that denominators involving pion energies in the reducible topologies of diagrams e1-e2, e6-e7, e8-e10, e13-e14, e20-e21, e22-e25 are expanded as in Eq. (82). The resulting contributions depend on the off-the-energy-shell prescription adopted for the non-static corrections to the OPE and TPE (reported in the Ch. 4), and OPEcontact potentials (reported in Ref. [23]). Different prescriptions lead to different formal expressions for these corrections as well as the accompanying weak axial current operators, which, however, are expected to be related to each other by unitary transformations. This unitary equivalence has been discussed in considerable detail in Ref. [23], where it was explicitly verified to hold in the case of the electromagnetic charge operator and is conjectured to hold also in the present case. The axial current operators derived below are obtained by adopting the $\nu=0$ prescription for the non-static corrections to the afore mentioned potentials, as given in Eqs. (142),(144), and (145) of the previous chapter. We note that in evaluating the contributions to diagrams e2 and e9 nonstatic corrections in the pion-pole term of the axial current at leading order need to be accounted for. These are obtained in Appendix H. 


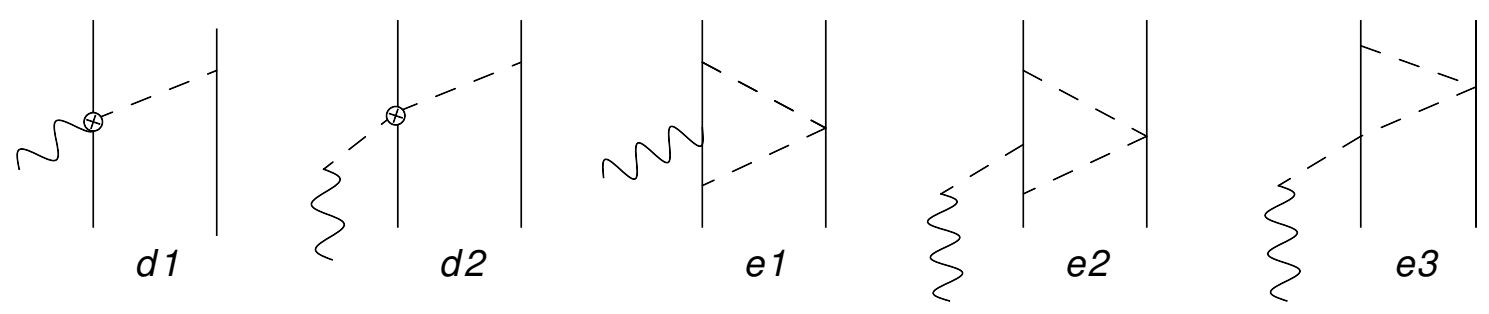

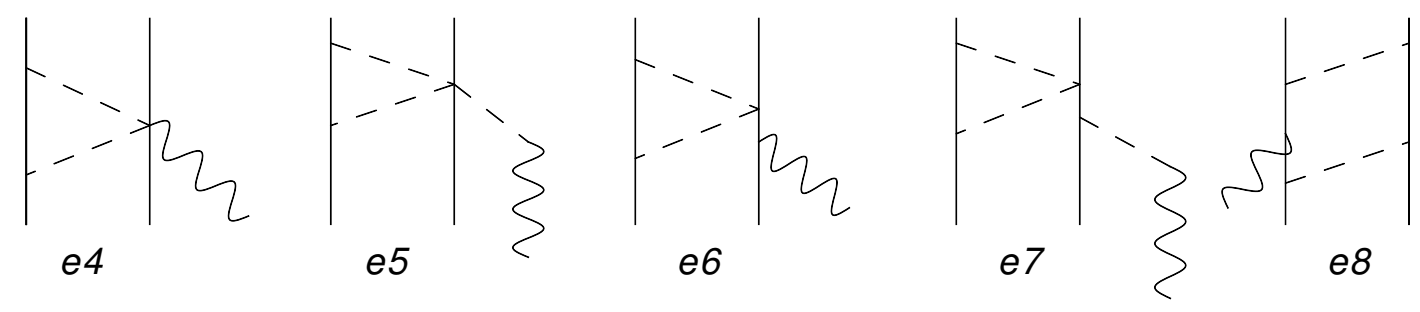
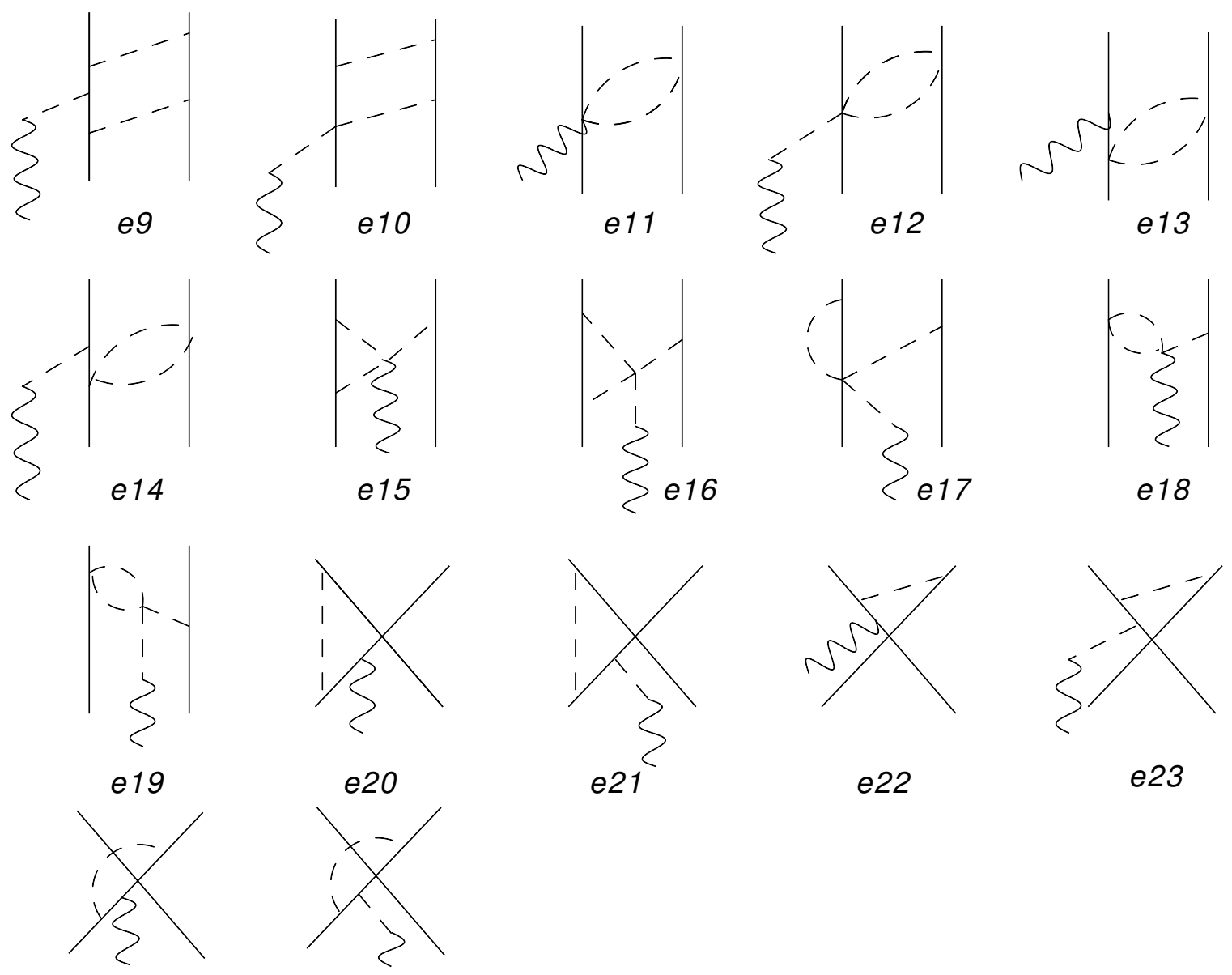

e24 e25

FIG. 10: Diagrams contributing to the OPE axial current operator at order $Q^{0}$ and to the MPE axial current at $\operatorname{order} Q$. Nucleons, pions, and axial fields are denoted by solid, dashed, and wavy lines, respectively. Crossed circle in diagrams d1 and d2 indicate vertices generated from chiral Hamiltonians at order $Q^{2}$. Only a single time ordering is shown for each topology. 
We find that the contributions of diagrams e3, e6-e7, e11-e14, e18-e25 vanish, while those of the remaining diagrams are given by

$$
\begin{aligned}
& \mathbf{j}_{5, a}^{(1)}(\mathrm{e} 1)=-\frac{g_{A}^{3}}{16 f_{\pi}^{4}} \tau_{2, a}\left[R_{i j}^{(2)}\left(\mathbf{k}_{2}\right) \sigma_{1 j}-\mathbf{k}_{2} R^{(0)}\left(k_{2}\right) \boldsymbol{\sigma}_{1} \cdot \mathbf{k}_{2}\right], \\
& \mathbf{j}_{5, a}^{(1)}(\mathrm{e} 2)=-\frac{\mathbf{q}}{q^{2}+m_{\pi}^{2}} \mathbf{q} \cdot \mathbf{j}_{5, a}^{(1)}(\mathrm{e} 1) \\
& \mathbf{j}_{5, a}^{(1)}(\mathrm{e} 4)=-\frac{g_{A}^{3}}{16 f_{\pi}^{4}} \tau_{2, a}\left[k_{1}^{2} R^{(0)}\left(k_{1}\right)-R^{(2)}\left(k_{1}\right)\right] \boldsymbol{\sigma}_{2}, \\
& \mathbf{j}_{5, a}^{(1)}(\mathrm{e} 5)=\frac{g_{A}^{3}}{32 f_{\pi}^{4}} \frac{\mathbf{q}}{q^{2}+m_{\pi}^{2}}\left[\tau_{2, a}\left[k_{1}^{2} R^{(0)}\left(k_{1}\right)-R^{(2)}\left(k_{1}\right)\right]\left[(10 \alpha-1) \boldsymbol{\sigma}_{2} \cdot \mathbf{k}_{2}+\boldsymbol{\sigma}_{2} \cdot \mathbf{k}_{1}\right]\right. \\
& \left.-\left(\boldsymbol{\tau}_{1} \times \boldsymbol{\tau}_{2}\right)_{a} R_{i j}^{(2)}\left(\mathbf{k}_{1}\right)\left(\boldsymbol{\sigma}_{1} \times \mathbf{k}_{1}\right)_{i} \sigma_{2, j}\right], \\
& \mathbf{j}_{5, a}^{(1)}(\mathrm{e} 8)=-\frac{g_{A}^{5}}{16 f_{\pi}^{4}}\left[\tau _ { 2 , a } \left[\left(\boldsymbol{\sigma}_{1} \times \mathbf{k}_{2}\right) \times \mathbf{k}_{2}\left[k_{2}^{2} S^{(0)}\left(k_{2}\right)-S^{(2)}\left(k_{2}\right)\right]\right.\right. \\
& \left.+\left[k_{2}^{2} S^{(2)}\left(k_{2}\right)-S^{(4)}\left(k_{2}\right)\right] \boldsymbol{\sigma}_{1}-\left[k_{2}^{2} S_{i j}^{(2)}\left(\mathbf{k}_{2}\right)-S_{i j}^{(4)}\left(\mathbf{k}_{2}\right)\right] \sigma_{1 j}\right] \\
& \left.-4 \tau_{1, a} \epsilon_{i j k} k_{2 j} S_{k l}^{(2)}\left(\mathbf{k}_{2}\right)\left(\boldsymbol{\sigma}_{2} \times \mathbf{k}_{2}\right)_{l}\right] \text {, } \\
& \mathbf{j}_{5, a}^{(1)}(\mathrm{e} 9)=-\frac{\mathbf{q}}{q^{2}+m_{\pi}^{2}} \mathbf{q} \cdot \mathbf{j}_{5, a}^{(1)}(\mathrm{e} 8), \\
& \mathbf{j}_{5, a}^{(1)}(\mathrm{e} 10)=\frac{g_{A}^{3}}{32 f_{\pi}^{4}} \frac{\mathbf{q}}{q^{2}+m_{\pi}^{2}}\left[\left(2 \tau_{2, a}-\tau_{1, a}\right)\left[k_{2}^{2} R^{(0)}\left(k_{2}\right)-R^{(2)}\left(k_{2}\right)\right] \boldsymbol{\sigma}_{1} \cdot \mathbf{k}_{2}\right. \\
& \left.+\left(\boldsymbol{\tau}_{1} \times \boldsymbol{\tau}_{2}\right)_{a} R_{i j}^{(2)}\left(\mathbf{k}_{1}\right)\left(\boldsymbol{\sigma}_{2} \times \mathbf{k}_{2}\right)_{i} \sigma_{1 j}\right], \\
& \mathbf{j}_{5, a}^{(1)}(\mathrm{e} 15)=\frac{g_{A}^{3}}{32 f_{\pi}^{4}}\left[\tau_{2, a}\left(10 \alpha \mathbf{q}-3 \mathbf{k}_{1}+\mathbf{k}_{2}\right)\left[k_{1}^{2} R^{(0)}\left(k_{1}\right)-R^{(2)}\left(k_{1}\right)\right]\right. \\
& \left.-4\left(\boldsymbol{\tau}_{1} \times \boldsymbol{\tau}_{2}\right)_{a} R_{i j}^{(2)}\left(\mathbf{k}_{1}\right)\left(\boldsymbol{\sigma}_{1} \times \mathbf{k}_{1}\right)_{j}\right] \frac{\boldsymbol{\sigma}_{2} \cdot \mathbf{k}_{2}}{\omega_{2}^{2}}, \\
& \mathbf{j}_{5, a}^{(1)}(\mathrm{e} 16)=\frac{g_{A}^{3}}{64 f_{\pi}^{4}} \tau_{2, a} \frac{\mathbf{q}}{q^{2}+m_{\pi}^{2}}\left[2\left(5 m_{\pi}^{2}+2 k_{1}^{2}+k_{2}^{2}+q^{2}\right)\left[k_{1}^{2} R^{(0)}\left(k_{1}\right)-R^{(2)}\left(k_{1}\right)\right]\right. \\
& +\left[k_{1}^{4} R^{(0)}\left(k_{1}\right)-R^{(4)}\left(k_{1}\right)\right]-20 \alpha\left(q^{2}+k_{2}^{2}+2 m_{\pi}^{2}\right)\left[k_{1}^{2} R^{(0)}\left(k_{1}\right)-R^{(2)}\left(k_{1}\right)\right] \\
& \left.+80 \alpha J_{12}\right] \frac{\boldsymbol{\sigma}_{2} \cdot \mathbf{k}_{2}}{\omega_{2}^{2}} \\
& +\frac{g_{A}^{3}}{16 f_{\pi}^{4}}\left(\boldsymbol{\tau}_{1} \times \boldsymbol{\tau}_{2}\right)_{a} \frac{\mathbf{q}}{q^{2}+m_{\pi}^{2}} R_{i j}^{(2)}\left(\mathbf{k}_{1}\right)\left(\boldsymbol{\sigma}_{1} \times \mathbf{k}_{1}\right)_{i}\left(\mathbf{k}_{2}+\mathbf{q}\right)_{j} \frac{\boldsymbol{\sigma}_{2} \cdot \mathbf{k}_{2}}{\omega_{2}^{2}}, \\
& \mathbf{j}_{5, a}^{(1)}(\mathrm{e} 17)=\frac{g_{A}^{3}}{8 f_{\pi}^{4}} \tau_{2, a} \frac{\mathbf{q}}{q^{2}+m_{\pi}^{2}}(1-10 \alpha) J_{12} \frac{\boldsymbol{\sigma}_{2} \cdot \mathbf{k}_{2}}{\omega_{2}^{2}},
\end{aligned}
$$

where the constants $J_{m n}$ are as in Eq. (392), and the loop functions $R_{i j}^{(n)}$ have been defined 
as

$$
\begin{aligned}
R^{(0)}(k) & =\int \frac{d \mathbf{p}}{(2 \pi)^{3}} \tilde{f}\left(\omega_{+}, \omega_{-}\right), \\
R^{(2)}(k) & =\int \frac{d \mathbf{p}}{(2 \pi)^{3}} p^{2} \tilde{f}\left(\omega_{+}, \omega_{-}\right), \\
R_{i j}^{(2)}(\mathbf{k}) & =\int \frac{d \mathbf{p}}{(2 \pi)^{3}} p_{i} p_{j} \tilde{f}\left(\omega_{+}, \omega_{-}\right), \\
R^{(4)}(k) & =\int \frac{d \mathbf{p}}{(2 \pi)^{3}} p^{4} \tilde{f}\left(\omega_{+}, \omega_{-}\right), \\
R_{i j}^{(4)}(\mathbf{k}) & =\int \frac{d \mathbf{p}}{(2 \pi)^{3}} p_{i} p_{j} p^{2} \tilde{f}\left(\omega_{+}, \omega_{-}\right),
\end{aligned}
$$

with

$$
\tilde{f}\left(\omega_{+}, \omega_{-}\right)=\frac{1}{\omega_{+}^{2} \omega_{-}^{2}}
$$

The loop functions $S_{i j}^{(n)}$ are defined similarly with $\tilde{f}\left(\omega_{+}, \omega_{-}\right)$replaced by

$$
\tilde{g}\left(\omega_{+}, \omega_{-}\right)=\frac{\omega_{+}^{2}+\omega_{-}^{2}}{\omega_{+}^{4} \omega_{-}^{4}}=-\frac{1}{4} \frac{d}{d m_{\pi}^{2}} \tilde{f}\left(\omega_{+}, \omega_{-}\right) .
$$

After dimensional regularization, we obtain

$$
\begin{aligned}
R^{(0)}(k)= & \frac{1}{16 \pi} \int_{0}^{1} d z \frac{1}{M(k, z)}, \\
R^{(2)}(k)= & -\frac{3}{4 \pi} \int_{0}^{1} d z\left[M(k, z)-\frac{1}{12} \frac{(z-\bar{z})^{2}}{M(k, z)} k^{2}\right] \\
R_{i j}^{(2)}(\mathbf{k})= & -\frac{1}{4 \pi} \int_{0}^{1} d z\left[\delta_{i j} M(k, z)-\frac{1}{4} \frac{(z-\bar{z})^{2}}{M(k, z)} k_{i} k_{j}\right] \\
R^{(4)}(k)= & \frac{5}{\pi} \int_{0}^{1} d z\left[M(k, z)^{3}-\frac{1}{2}(z-\bar{z})^{2} M(k, z) k^{2}+\frac{1}{80} \frac{(z-\bar{z})^{4}}{M(k, z)} k^{4}\right] \\
R_{i j}^{(4)}(\mathbf{k})= & \frac{5}{3 \pi} \int_{0}^{1} d z\left[\delta_{i j}\left[M(k, z)^{3}-\frac{3}{20}(z-\bar{z})^{2} M(k, z) k^{2}\right]\right. \\
& \left.-\frac{21}{20}\left[(z-\bar{z})^{2} M(k, z)-\frac{1}{28} \frac{(z-\bar{z})^{4}}{M(k, z)} k^{2}\right] k_{i} k_{j}\right]
\end{aligned}
$$

where

$$
M(k, z)=\sqrt{z \bar{z} k^{2}+m_{\pi}^{2}}, \quad \bar{z}=1-z .
$$

The regularized $S_{i j}^{(n)}(k)$ loop functions easily follow from Eq. (193). Inserting these relations into the equations above, and noting that the $\alpha$ dependence cancels out upon summing 
the contributions of diagrams e5, e15, e16, and e17, we obtain the expressions reported in Appendix I. No divergencies occur in these loop corrections at order $Q$, consistently with the fact that there are no contact terms in the axial current at this order. Contributions coming from $\mathcal{L}_{\pi N}^{(3)}$, proportional to the LECs $d_{i}$ 's, that enter through topologies $\mathrm{d} 1$ and $\mathrm{d} 2$, turn out to vanish.

\subsection{LOOP CORRECTIONS TO ONE-PION EXCHANGE}

In this section we discuss how to calculate the loop corrections to the one-pion exchange (OPE) operators, for both the axial charge and axial current. The calculation of the OPE axial charge is somewhat involved because the rather large number of nonvanishing topologies. For the axial current the situation is considerably simpler.

\subsubsection{COUNTERTERMS}

We now proceed to renormalize the loop corrections to the OPE axial charge operator which come in at order $Q$. The renormalization of nucleon and pion masses, and field rescaling factors $Z_{\pi}$ and $Z_{N}$, has been carried out in Appendix D. The only other ingredients which are needed are the relations between the renormalized (physical) pion decay constant $f_{\pi}^{r}$ and nucleon axial coupling constant $g_{A}^{r}$ and their respective bare quantities. These relations have been derived in Appendix F. We report them here

$$
\begin{aligned}
f_{\pi} & =f_{\pi}^{r}\left(1-\frac{m_{\pi}^{r 2} l_{4}}{f_{\pi}^{r 2}}+\frac{J_{01}}{2 f_{\pi}^{r 2}}\right), \\
g_{A} & =g_{A}^{r}\left[1+\frac{1}{2 f_{\pi}^{r 2}} J_{01}+\frac{g_{A}^{r 2}}{3 f_{\pi}^{r 2}} J_{13}-\frac{4 m_{\pi}^{r 2}}{g_{A}^{r}} d_{16}\right],
\end{aligned}
$$

where the constants $J_{m n}$ are defined in Eq. (392).

Next we need the set of relevant counter-terms. As illustrated in Appendix C, the total Lagrangian, including the axial field $A_{a}^{\mu}$, can be written as

$$
\begin{aligned}
\mathcal{L}= & \bar{N}^{r}\left(i \not \partial-m^{r}+\Gamma_{a}^{0 \prime} \partial_{0} \pi_{a}^{r}+\Lambda_{a}^{i \prime} \partial_{i} \pi_{a}^{r}+\Delta^{\prime}\right) N^{r} \\
& +\frac{1}{2}\left(\partial^{0} \pi_{a}^{r} G_{a b}^{\prime} \partial_{0} \pi_{b}^{r}+\partial^{i} \pi_{a}^{r} \widetilde{G}_{a b}^{\prime} \partial_{i} \pi_{b}^{r}-m_{\pi}^{r 2} \pi_{a}^{r} H_{a b}^{\prime} \pi_{b}^{r}\right)-f_{\pi} A_{a}^{\mu} F_{a b}^{\prime} \partial_{\mu} \pi_{b}^{r} \\
& +\delta m \bar{N}^{r} N^{r}+\delta Z_{N} \bar{N}^{r}\left(i \gamma^{\mu} \partial_{\mu}-m^{r}\right) N^{r}+\frac{\delta m_{\pi}^{2}}{2} \pi_{a}^{r} \pi_{a}^{r},
\end{aligned}
$$

which is expressed in terms of renormalized fields and masses, but bare $g_{A}, f_{\pi}$ and other LECs. This Lagrangian has essentially the same form as the bare one in Eq. (68) (the primed 
quantities are defined in Appendix C), and leads to a similar interaction Hamiltonian as in Eq. (75),

$$
\begin{aligned}
\mathcal{H}_{I}= & \mathcal{H}_{I}[\text { Eq. (75) with primed quantities and renormalized fields and masses ] } \\
& -\delta m \bar{N}^{r} N^{r}-\delta Z_{N} \bar{N}^{r}\left(i \gamma^{i} \partial_{i}-m^{r}\right) N^{r}-\frac{\delta m_{\pi}^{2}}{2} \pi_{a}^{r} \pi_{a}^{r} .
\end{aligned}
$$

In addition to the vertices listed in Appendix B, this Hamiltonian generates vertices corresponding to the set of counter-terms in Eqs. (447)-(453), explicit expressions for which follow from those in Appendix B.

\subsubsection{RENORMALIZATION OF OPE AXIAL CHARGE}

We begin by discussing the non-pion-pole contributions illustrated in Fig. 11. In diagrams g2, g4, g6, g8, g11, and g14, the solid dot represents the interaction $-\delta m-4 m_{\pi}^{r 2} c_{1}$, where $\delta m$ is the nucleon mass counter-term. The contributions associated with diagrams g1-g2, g3-g4, g5-g6, g7-g8, g9-g11, and g12-g14 represent the renormalization of nucleon external lines and, with the choice of $\delta m$ in Eq. (131) they vanish.

Next, the solid square in diagrams g16, g18, and g20 represents the interaction

$$
\begin{aligned}
H_{2 \pi}^{(4) \prime}= & -\int \mathrm{d} \mathbf{x}\left(\frac{m_{\pi}^{r 2} l_{4}}{f_{\pi}^{2}}+\frac{\delta Z_{\pi}}{2}\right)\left(\boldsymbol{\Pi}^{r} \cdot \boldsymbol{\Pi}^{r}+\partial^{i} \boldsymbol{\pi}^{r} \cdot \partial_{i} \boldsymbol{\pi}^{r}\right) \\
& +\int \mathrm{d} \mathbf{x}\left[\frac{m_{\pi}^{r 4}\left(l_{3}+l_{4}\right)}{f_{\pi}^{2}}+\frac{m_{\pi}^{r 2}}{2} \delta Z_{\pi}-\frac{\delta m_{\pi}^{2}}{2}\right] \boldsymbol{\pi}^{r} \cdot \boldsymbol{\pi}^{r}
\end{aligned}
$$

with vertex (in the convention of Appendix B)

$$
\begin{aligned}
\left\langle 0\left|H_{2 \pi}^{(4) \prime}\right| \mathbf{k}_{1}, a_{1} ; \mathbf{k}_{2}, a_{2}\right\rangle= & \delta_{a_{1}, a_{2}}\left[\left(\frac{2 m_{\pi}^{r 2} l_{4}}{f_{\pi}^{2}}+\delta Z_{\pi}\right)\left(\omega_{1} \omega_{2}-\mathbf{k}_{1} \cdot \mathbf{k}_{2}\right)\right. \\
& \left.+\frac{2 m_{\pi}^{r 4}\left(l_{3}+l_{4}\right)}{f_{\pi}^{2}}+m_{\pi}^{r 2} \delta Z_{\pi}-\delta m_{\pi}^{2}\right]
\end{aligned}
$$

With $\delta Z_{\pi}$ and $\delta m_{\pi}^{2}$ as given in Eq. (131), the contributions of diagrams g15-g20 cancel out. 

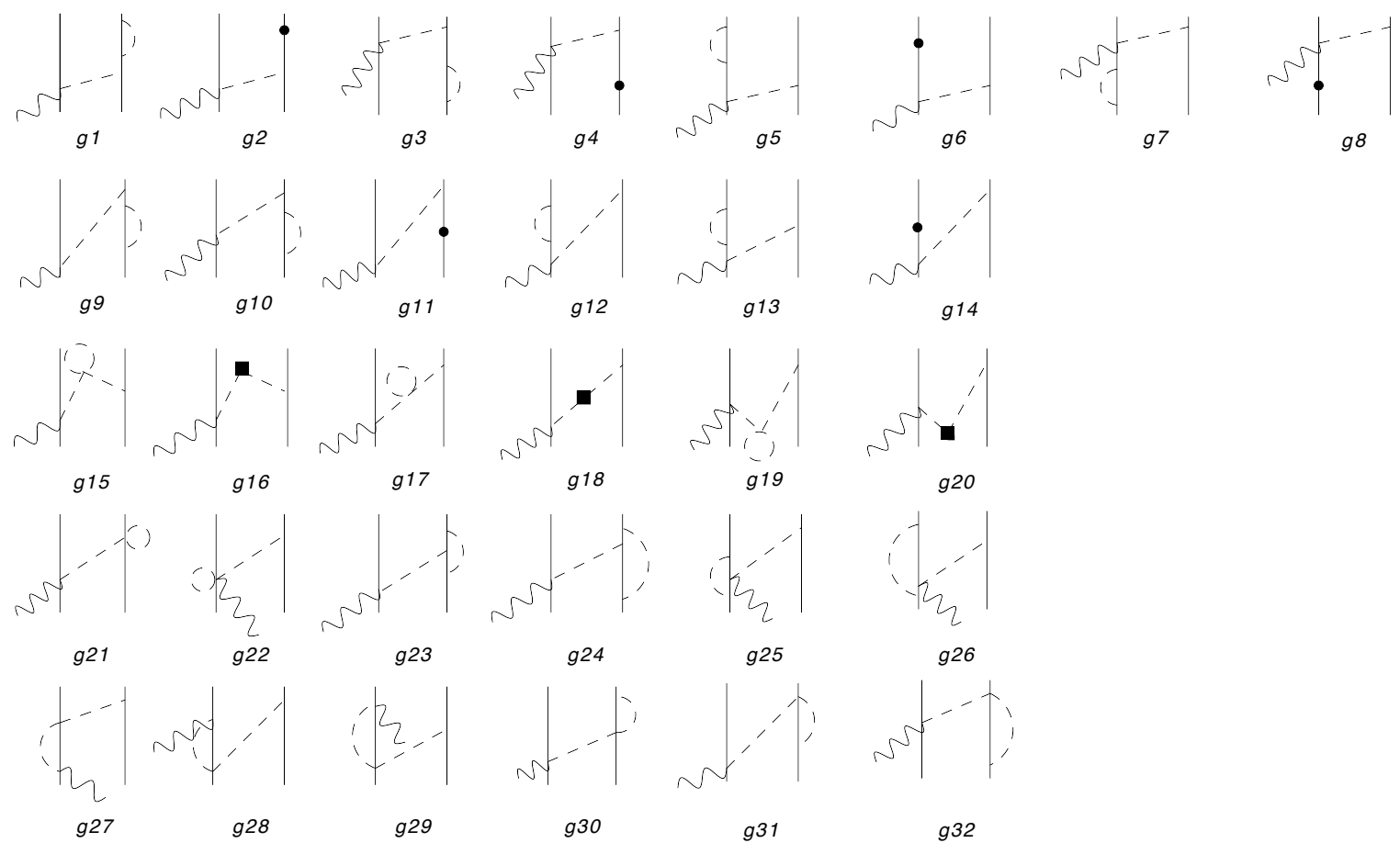

FIG. 11: Half of the possible time-ordered non-pole corrections to the OPE axial charge at order $Q$. Nucleons, pions, and axial fields are denoted by solid, dashed, and wavy lines, respectively. See text for further explanations.

The remaining loop contributions in diagrams g21-g29 are given by

$$
\begin{aligned}
\rho_{5, a}^{(1)}(\mathrm{g} 21) & =\rho_{5, a}^{(-1)}(\mathrm{a} 1) \frac{1}{4 f_{\pi}^{2}}(1-10 \alpha) J_{01}, \\
\rho_{5, a}^{(1)}(\mathrm{g} 22) & =\rho_{5, a}^{(-1)}(\mathrm{a} 1) \frac{5}{8 f_{\pi}^{2}}(1-4 \alpha) J_{01}, \\
\rho_{5, a}^{(1)}(\mathrm{g} 23+\mathrm{g} 24) & =\rho_{5, a}^{(-1)}(\mathrm{a} 1) \frac{g_{A}^{2}}{24 f_{\pi}^{2}} J_{13}, \\
\rho_{5, a}^{(1)}(\mathrm{g} 25+\mathrm{g} 26) & =-\rho_{5, a}^{(-1)}(\mathrm{a} 1) \frac{g_{A}^{2}}{8 f_{\pi}^{2}} J_{13}, \\
\rho_{5, a}^{(1)}(\mathrm{g} 27+\mathrm{g} 28+\mathrm{g} 29) & =\rho_{5, a}^{(-1)}(\mathrm{a} 1) \frac{1}{4 f_{\pi}^{2}} J_{01},
\end{aligned}
$$

while those in diagrams g30-g32 vanish identically. Here $\rho_{5, a}^{(-1)}(a 1)$ is defined as in Eq. (155).

Finally, one needs to include the contributions due to the interactions coming from pion and nucleon field redefinitions. These contributions enter through the $\pi N N$ vertex generated by the Hamiltonian denoted as $H_{\pi N N}^{(3) \prime}$, reported in Eq. (473), and through the $\pi N N A$ vertex 
generated by the following Hamiltonian

$$
H_{\pi N N A}^{(3) \prime}=-\left(\delta Z_{N}+\delta Z_{\pi} / 2\right) \frac{1}{4 f_{\pi}} \int d \mathbf{x} \bar{N}^{r} \mathbf{A}_{0} \cdot\left(\boldsymbol{\tau} \times \boldsymbol{\pi}^{r}\right) \gamma_{0} N^{r} .
$$

They lead to the correction

$$
\left[2 \delta Z_{N}+\delta Z_{\pi}+\frac{2 m_{\pi}^{r 2}}{g_{A}}\left(2 d_{16}-d_{18}\right)\right] \rho_{5, a}^{(-1)}(\mathrm{a} 1) .
$$

Thus, the sum of the order $Q$ corrections to the axial charge from non-pole contributions, denoted as $\rho_{5, a}^{(1)}(\mathrm{npp})$, reads

$$
\begin{aligned}
\rho_{5, a}^{(1)}(\mathrm{npp})= & \rho_{5, a}^{(-1)}(\mathrm{a} 1)\left[\frac{1}{f_{\pi}^{2}}\left(\frac{9}{8}-5 \alpha\right) J_{01}-\frac{g_{A}^{2}}{12 f_{\pi}^{2}} J_{13}+2 \delta Z_{N}\right. \\
& \left.+\delta Z_{\pi}+\frac{2 m_{\pi}^{r 2}}{g_{A}}\left(2 d_{16}-d_{18}\right)\right]
\end{aligned}
$$

which, which after insertion of $\delta Z_{N}$ and $\delta Z_{\pi}$, is expressed as

$$
\begin{aligned}
\rho_{5, a}^{(1)}(\mathrm{npp})= & i \frac{g_{A}^{r}}{8 f_{\pi}^{r 2}}\left(\boldsymbol{\tau}_{1} \times \boldsymbol{\tau}_{2}\right)_{a} \boldsymbol{\sigma}_{2} \cdot \mathbf{k}_{2} \frac{1}{\omega_{2}^{2}}\left[\frac{5}{8 f_{\pi}^{r 2}} J_{01}-\frac{5 g_{A}^{r 2}}{6 f_{\pi}^{r 2}} J_{13}\right. \\
& \left.-\frac{2 m_{\pi}^{r 2}}{f_{\pi}^{r 2}} l_{4}+\frac{2 m_{\pi}^{r 2}}{g_{A}^{r}}\left(2 d_{16}-d_{18}\right)\right]
\end{aligned}
$$

where the bare $g_{A}$ and $f_{\pi}$ have been replaced by their respective renormalized valuesthis replacement is correct to the order of interest here. The complete non-pion-pole axial charge, denoted as $\rho_{5, a}^{\mathrm{OPE}}(\mathrm{npp})$ below, results from the sum of the leading-order contribution in Eq. (155) with the ratio $g_{A} / f_{\pi}^{2}$ replaced by its renormalized value explictly derived in Appendix E as

$$
\frac{g_{A}}{f_{\pi}^{2}}=\frac{g_{A}^{r}}{f_{\pi}^{r 2}}\left[1-\frac{1}{2 f_{\pi}^{r 2}} J_{01}+\frac{g_{A}^{r 2}}{3 f_{\pi}^{r 2}} J_{13}+\frac{2 m_{\pi}^{r 2}}{f_{\pi}^{r 2}} l_{4}-\frac{2 m_{\pi}^{r 2}}{g_{A}^{r}}\left(2 d_{16}-d_{18}\right)\right],
$$

and the contribution $\rho_{5, a}^{(1)}(\mathrm{npp})$. We obtain for this sum

$$
\rho_{5, a}(\mathrm{OPE}, \mathrm{npp})=i \frac{g_{A}^{r}}{8 f_{\pi}^{r 2}}\left(\boldsymbol{\tau}_{1} \times \boldsymbol{\tau}_{2}\right)_{a} \boldsymbol{\sigma}_{2} \cdot \mathbf{k}_{2} \frac{1}{\omega_{2}^{2}}\left(1+\frac{1}{8 f_{\pi}^{r 2}} J_{01}-\frac{g_{A}^{r 2}}{2 f_{\pi}^{r 2}} J_{13}\right) .
$$



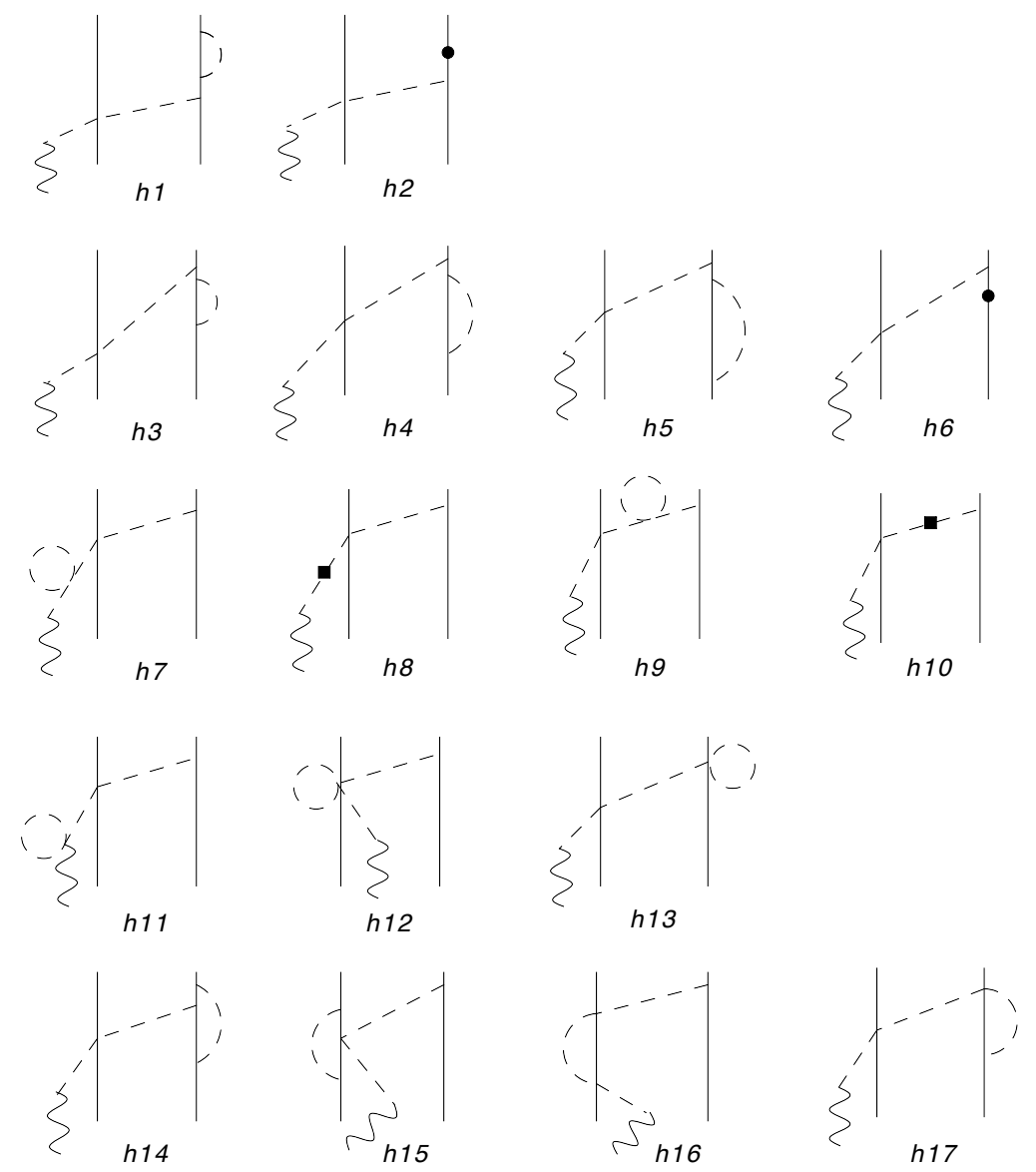

FIG. 12: Representative diagrams for each of the relevant classes contributing to pole corrections to the OPE axial charge at order $Q$. Nucleons, pions, and axial fields are denoted by solid, dashed, and wavy lines, respectively. More than a single time ordering is shown for some of the diagrams.

The diagrams describing the pion-pole corrections are illustrated in Fig. 12 (only representative diagrams for each of the relevant classes are drawn for brevity), and are similar to those in Fig. 11. A slightly more complicated analysis along the lines illustrated above leads to a pion-pole OPE axial charge, denoted $\rho_{5, a}^{(1)}(\mathrm{pp})$, given by

$$
\rho_{5, a}(\mathrm{OPE}, \mathrm{pp})=i \frac{g_{A}^{r}}{8 f_{\pi}^{r 2}}\left(\boldsymbol{\tau}_{1} \times \boldsymbol{\tau}_{2}\right)_{a} \boldsymbol{\sigma}_{2} \cdot \mathbf{k}_{2} \frac{1}{\omega_{2}^{2}}\left(1-\frac{1}{8 f_{\pi}^{r^{2}}} J_{01}-\frac{g_{A}^{r^{2}}}{2 f_{\pi}^{r^{2}}} J_{13}\right) .
$$

The sum of the npp and pp contributions evaluated in dimensional regularization is

$$
\begin{aligned}
\rho_{5, a}(\mathrm{OPE}, \mathrm{npp}+\mathrm{pp}) & =i \frac{g_{A}^{r}}{8 f_{\pi}^{r 2}}\left(\boldsymbol{\tau}_{1} \times \boldsymbol{\tau}_{2}\right)_{a} \boldsymbol{\sigma}_{2} \cdot \mathbf{k}_{2} \frac{1}{\omega_{2}^{2}}\left(2-\frac{g_{A}^{r 2}}{f_{\pi}^{r 2}} J_{13}\right) \\
& =\rho_{5, a}^{(-1)}(\mathrm{a} 1)\left[2-\frac{3 m_{\pi}^{r 2}}{8 \pi^{2} f_{\pi}^{r 2}} g_{A}^{r 2}\left(d_{\epsilon}-\frac{1}{3}\right)\right]
\end{aligned}
$$


where $d_{\epsilon}$ is defined in Eq. (110).

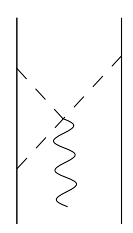

$f 1$

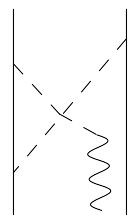

$f 2$

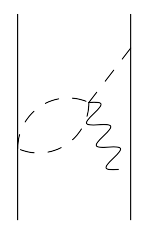

f3

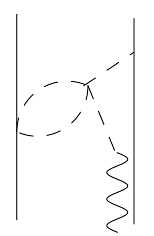

$f 4$

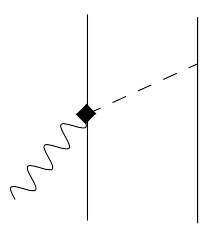

f5

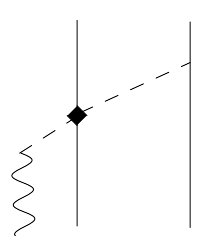

f6

FIG. 13: Additional loop and tree-level corrections of order $Q$ to the OPE axial charge. Nucleons, pions, and axial fields are denoted by solid, dashed, and wavy lines, respectively. Only a single time ordering is shown for each topology. See text for further explanations.

There are additional loop corrections to the OPE axial charge, see Fig. 13. Their contributions are obtained as

$$
\begin{aligned}
\rho_{5, a}^{(1)}(\mathrm{f} 1+\mathrm{f} 2) & =-\frac{g_{A}^{r 2}}{2 f_{\pi}^{r 2}} \rho_{5, a}^{(-1)}(\mathrm{a} 1)\left[k_{1}^{2} I^{(0)}\left(k_{1}\right)-I^{(2)}\left(k_{1}\right)\right], \\
\rho_{5, a}^{(1)}(\mathrm{f} 3+\mathrm{f} 4) & =-\frac{1}{8 f_{\pi}^{r 2}} \rho_{5, a}^{(-1)}(a 1) L\left(k_{1}\right),
\end{aligned}
$$

where $\rho_{5, a}^{(-1)}$ (a1) is again defined as in Eq. (155), except that $g_{A}$ and $f_{\pi}$ are replaced by their renormalized values $g_{A}^{r}$ and $f_{\pi}^{r}$. The loop function $I^{(0)}(k), I^{(2)}(k)$ and $L(k)$ have been defined in Eqs. (102)-(104). Evaluation in dimensional regularization leads to

$$
\begin{aligned}
\rho_{5, a}^{(1)}(\mathrm{f} 1+\mathrm{f} 2)= & \rho_{5, a}^{(-1)}(\mathrm{a} 1) \frac{g_{A}^{r 2}}{48 \pi^{2} f_{\pi}^{r 2}}\left[\frac{s_{1}}{k_{1}} \ln \left(\frac{s_{1}+k_{1}}{s_{1}-k_{1}}\right)\left(5 k_{1}^{2}+8 m_{\pi}^{r 2}\right)\right. \\
& \left.+k_{1}^{2}\left(5 d_{\epsilon}-\frac{13}{3}\right)+18 m_{\pi}^{r 2}\left(d_{\epsilon}-\frac{2}{9}\right)\right], \\
\rho_{5, a}^{(1)}(\mathrm{f} 3+\mathrm{f} 4)= & \rho_{5, a}^{(-1)}(\mathrm{a} 1) \frac{1}{48 \pi^{2} f_{\pi}^{r 2}}\left[\frac{s_{1}^{3}}{k_{1}} \ln \frac{s_{1}+k_{1}}{s_{1}-k_{1}}-8 m_{\pi}^{r 2}+k_{1}^{2}\left(d_{\epsilon}-\frac{5}{3}\right)\right] .
\end{aligned}
$$

We also need to account for tree-level contributions of order $Q$ originating from the vertices $2 \pi N N$ and $N N \pi A^{0}$ in Eqs. (404) and (419), denoted by the solid diamonds in Fig. 13. They can be written as

$$
\begin{aligned}
\rho_{5, a}^{(1)}(\mathrm{f} 5+\mathrm{f} 6)= & 2 \rho_{5, a}^{(-1)}(\mathrm{a} 1)\left(\widetilde{d}_{1} k_{1}^{2}+\widetilde{d}_{2} k_{2}^{2}+\widetilde{d}_{3} q^{2}+\widetilde{d}_{4} m_{\pi}^{r 2}\right) \\
& +i \frac{g_{A}^{r}}{2 f_{\pi}^{r 2}} \widetilde{d}_{5} \tau_{2, a} \boldsymbol{\sigma}_{1} \cdot\left(\mathbf{q} \times \mathbf{k}_{2}\right) \boldsymbol{\sigma}_{2} \cdot \mathbf{k}_{2} \frac{1}{\omega_{2}^{2}}
\end{aligned}
$$


where we have introduced the following combinations of LECs

$$
\begin{aligned}
& \tilde{d}_{1}=2 d_{2}+d_{6}, \\
& \tilde{d}_{2}=4 d_{1}+2 d_{2}+4 d_{3}-d_{6}, \\
& \widetilde{d}_{3}=-2 d_{2}+d_{6}, \\
& \widetilde{d}_{4}=4 d_{1}+4 d_{2}+4 d_{3}+8 d_{5}, \\
& \widetilde{d}_{5}=d_{15}+2 d_{23},
\end{aligned}
$$

and the $d_{i}$ are LECs present in the subleading Lagrangian $\mathcal{L}_{\pi N}^{(3)}$. The divergent parts of the $d_{i}$ 's (and hence $\widetilde{d}_{i}$ 's) have been identified in the heavy-baryon formalism, without considering any specific process, with the background-field and heat-kernel methods, see Ref. [53] and references therein. We report below the expressions for these divergent parts from Table 4 of that work:

$$
d_{i}=\frac{\beta_{i}}{f_{\pi}^{2}} \lambda+d_{i}^{r}(\mu)
$$

where, in the conventions adopted in the present work,

$$
\begin{aligned}
\lambda & =\frac{1}{32 \pi^{2}}\left(d_{\epsilon}+\ln \frac{\mu^{2}}{m_{\pi}^{2}}\right), \\
d_{i}^{r}(\mu) & =\frac{\beta_{i}}{32 \pi^{2} f_{\pi}^{2}} \ln \frac{m_{\pi}^{2}}{\mu^{2}}+d_{i}^{r}\left(m_{\pi}\right) .
\end{aligned}
$$

The $\beta_{i}$ functions of interest here are

$$
\begin{aligned}
& \beta_{1}=-\frac{g_{A}^{4}}{6}, \quad \beta_{2}=-\frac{1}{12}-\frac{5 g_{A}^{2}}{12}, \quad \beta_{3}=\frac{1}{2}+\frac{g_{A}^{4}}{6}, \\
& \beta_{5}=\frac{1}{24}+\frac{5 g_{A}^{2}}{24}, \quad \beta_{6}=-\frac{1}{6}-\frac{5 g_{A}^{2}}{6}, \quad \beta_{15}=\beta_{23}=0,
\end{aligned}
$$

and $\beta_{5}$ is from Ref. [53] which corresponds to our choice of operator basis in $\mathcal{L}_{\pi \pi}^{(4)}$. For the combinations $\widetilde{d}_{i}$ above we obtain

$$
\begin{aligned}
& \tilde{d}_{1}=-\frac{1}{96 \pi^{2} f_{\pi}^{2}}\left(1+5 g_{A}^{2}\right) d_{\epsilon}+\widetilde{d}_{1}^{r}, \\
& \widetilde{d}_{2}=\frac{1}{16 \pi^{2} f_{\pi}^{2}} d_{\epsilon}+\widetilde{d}_{2}^{r}, \\
& \widetilde{d}_{4}=\frac{1}{16 \pi^{2} f_{\pi}^{2}} d_{\epsilon}+\widetilde{d}_{4}^{r},
\end{aligned}
$$

and $\widetilde{d}_{3}=\widetilde{d}_{3}^{r}$ and $\widetilde{d}_{5}=\widetilde{d}_{5}^{r}$. We observe that the divergence proportional to $m_{\pi}^{2}$ from loop corrections in $\rho_{5, a}^{\mathrm{OPE}}(\mathrm{npp}+\mathrm{pp})$ cancels exactly that present in $\mathrm{f} 1+\mathrm{f} 2$. Next, the divergent 
part of $\widetilde{d}_{1}$ cancels exactly the term proportional to $k_{1}^{2} d_{\epsilon}$ present in $\mathrm{f} 1+\mathrm{f} 2$ and $\mathrm{f} 3+\mathrm{f} 4$. The divergent parts of $\widetilde{d}_{2}$ and $\widetilde{d}_{4}$ are the same, and therefore can be reabsorbed in the LEC $z_{2}$ multiplying the contact term $\mathrm{O}_{2}$. Those of $\widetilde{d}_{3}$ and $\widetilde{d}_{5}$ vanish, which is consistent with the fact that there are no divergencies proportional to $q^{2}$ or in the operator multiplying $\widetilde{d}_{5}$.

Combining Eqs. (216), (217), (221), (222), and (223), we then find that the renormalized OPE contributions up to order $Q$ included read as

$$
\begin{aligned}
\rho_{5, a}(\mathrm{OPE})= & i \frac{g_{A}^{r}}{4 f_{\pi}^{r 2}}\left(\boldsymbol{\tau}_{1} \times \boldsymbol{\tau}_{2}\right)_{a} \boldsymbol{\sigma}_{2} \cdot \mathbf{k}_{2} \frac{1}{\omega_{2}^{2}}\left[1+\frac{g_{A}^{r 2}}{96 \pi^{2} f_{\pi}^{r 2}}\left[\left(5 k_{1}^{2}+8 m_{\pi}^{r 2}\right) \frac{s_{1}}{k_{1}} \ln \frac{s_{1}+k_{1}}{s_{1}-k_{1}}\right.\right. \\
& \left.-\frac{13}{3} k_{1}^{2}+2 m_{\pi}^{2}\right]+\frac{1}{96 \pi^{2} f_{\pi}^{r 2}}\left(\frac{s_{1}^{3}}{k_{1}} \ln \frac{s_{1}+k_{1}}{s_{1}-k_{1}}-\frac{5}{3} k_{1}^{2}-8 m_{\pi}^{r 2}\right)+\left(\widetilde{d}_{1}^{r} k_{1}^{2}+\widetilde{d}_{2}^{r} k_{2}^{2}\right. \\
& \left.\left.+\widetilde{d}_{3}^{r} q^{2}+\widetilde{d}_{4}^{r} m_{\pi}^{r 2}\right)\right]+i \frac{g_{A}^{r}}{2 f_{\pi}^{r 2}} \widetilde{d}_{5}^{r} \tau_{2, a} \boldsymbol{\sigma}_{1} \cdot\left(\mathbf{q} \times \mathbf{k}_{2}\right) \boldsymbol{\sigma}_{2} \cdot \mathbf{k}_{2} \frac{1}{\omega_{2}^{2}} .
\end{aligned}
$$

\subsubsection{OPE AXIAL CURRENT}

In this case there are no contributions of the type shown in Fig. 11 at order $Q$ and the only loop corrections to the OPE axial current are those in Figs. 12 and 14. However the contributions of diagrams h1-h17 are easily seen to vanish, while those of diagrams m1-m2 are obtained as

$$
\begin{aligned}
& \mathbf{j}_{5, a}^{(1)}(\mathrm{m} 1)=-\frac{g_{A}^{r 5}}{96 f_{\pi}^{r 4}} J_{14}\left[9 \tau_{2, a} \mathbf{k}_{2}-\left(\boldsymbol{\tau}_{1} \times \boldsymbol{\tau}_{2}\right)_{a}\left(\boldsymbol{\sigma}_{1} \times \mathbf{k}_{2}\right)\right] \boldsymbol{\sigma}_{2} \cdot \mathbf{k}_{2} \frac{1}{\omega_{2}^{2}}, \\
& \mathbf{j}_{5, a}^{(1)}(\mathrm{m} 2)=-\frac{\mathbf{q}}{q^{2}+m_{\pi}^{2}} \mathbf{q} \cdot \mathbf{j}_{5, a}^{(1)}(\mathrm{m} 1) .
\end{aligned}
$$

In dimensional regularization we find the finite result

$$
\mathbf{j}_{5, a}^{(1)}(\mathrm{m} 1)=\frac{g_{A}^{r 5} m_{\pi}^{r}}{256 \pi f_{\pi}^{r 4}}\left[9 \tau_{2, a} \mathbf{k}_{2}-\left(\boldsymbol{\tau}_{1} \times \boldsymbol{\tau}_{2}\right)_{a}\left(\boldsymbol{\sigma}_{1} \times \mathbf{k}_{2}\right)\right] \boldsymbol{\sigma}_{2} \cdot \mathbf{k}_{2} \frac{1}{\omega_{2}^{2}} .
$$

No renormalization is necessary in this case. We emphasize again that loop corrections to diagrams d1-d2 of Fig. 10 enter at order $Q^{2}$ and are beyond the scope of the present Thesis. 


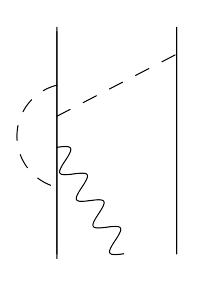

$m 1$

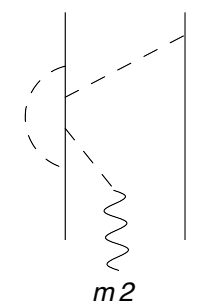

$m 2$

FIG. 14: The only non-vanishing loop corrections to the OPE axial current. Nucleons, pions, and axial fields are denoted by solid, dashed, and wavy lines, respectively. Only a single time ordering is shown for each topology.

\subsection{SUMMARY OF AXIAL CHARGE AND CURRENT UP TO ONE LOOP}

In this section we collect, for ease of reference, all expressions for $\rho_{5, a}$ and $\mathbf{j}_{5, a}$. All couplings and masses are intended to be renormalized (the superscript $r$ is dropped for simplicity), and we label the various contributions by their power counting $\mathrm{N} n \mathrm{LO}$, where $n=1,2,3$, and 4 and $\mathrm{N} n \mathrm{LO}$ means the given contribution is suppressed by $\left(Q / \Lambda_{\chi}\right)^{n}$ relative to the leading order (LO) term. The one-body axial current operators read

$$
\begin{aligned}
\mathbf{j}_{5, a}^{\mathrm{LO}}=-\frac{g_{A}}{2} \tau_{1, a} & {\left[\boldsymbol{\sigma}_{1}-\frac{\mathbf{q}}{q^{2}+m_{\pi}^{2}} \boldsymbol{\sigma}_{1} \cdot \mathbf{q}\right] } \\
\mathbf{j}_{5, a}^{\mathrm{N} 2 \mathrm{LO}}=\frac{g_{A}}{4 m^{2}} \tau_{1, a} & {\left[\mathbf{R}\left(\boldsymbol{\sigma}_{1}, \mathbf{k}_{1}, \mathbf{K}_{1}\right)-\frac{\mathbf{q}}{q^{2}+m_{\pi}^{2}} \mathbf{q} \cdot \mathbf{R}\left(\boldsymbol{\sigma}_{1}, \mathbf{k}_{1}, \mathbf{K}_{1}\right)\right.} \\
& \left.-2 \frac{\mathbf{q}}{q^{2}+m_{\pi}^{2}} \boldsymbol{\sigma}_{1} \cdot \mathbf{K}_{1} \mathbf{k}_{1} \cdot \mathbf{K}_{1}\right]
\end{aligned}
$$

where

$$
\mathbf{R}\left(\boldsymbol{\sigma}_{1}, \mathbf{k}_{1}, \mathbf{K}_{1}\right)=K_{1}^{2} \boldsymbol{\sigma}_{1}+\frac{i}{2} \mathbf{k}_{1} \times \mathbf{K}_{1}-\boldsymbol{\sigma}_{1} \cdot \mathbf{K}_{1} \mathbf{K}_{1}+\frac{1}{4} \boldsymbol{\sigma}_{1} \cdot \mathbf{k}_{1} \mathbf{k}_{1} .
$$

The OPE contribution to the axial charge reads

$$
\rho_{5, a}^{\mathrm{N} 1 \mathrm{LO}}(\mathrm{OPE})=i \frac{g_{A}}{4 f_{\pi}^{2}}\left(\boldsymbol{\tau}_{1} \times \boldsymbol{\tau}_{2}\right)_{a} \boldsymbol{\sigma}_{2} \cdot \mathbf{k}_{2} \frac{1}{\omega_{2}^{2}} .
$$

The OPE contributions to the axial current read

$$
\begin{aligned}
\widetilde{\mathbf{j}}_{5, a}(\mathrm{OPE})= & \mathbf{j}_{5, a}(\mathrm{OPE})-\frac{\mathbf{q}}{q^{2}+m_{\pi}^{2}} \mathbf{q} \cdot \mathbf{j}_{5, a}(\mathrm{OPE})-\frac{g_{A}}{2 f_{\pi}^{2}} \frac{\mathbf{q}}{q^{2}+m_{\pi}^{2}}\left[4 m_{\pi}^{2} c_{1} \tau_{2, a}\right. \\
& \left.-\frac{i}{2 m}\left(\boldsymbol{\tau}_{1} \times \boldsymbol{\tau}_{2}\right)_{a}\left(\mathbf{K}_{1} \cdot \mathbf{k}_{1}+\mathbf{K}_{2} \cdot \mathbf{k}_{2}\right)\right] \boldsymbol{\sigma}_{2} \cdot \mathbf{k}_{2} \frac{1}{\omega_{2}^{2}}
\end{aligned}
$$


where, for convenience, we have defined $\mathbf{j}_{5, a}(\mathrm{OPE})$ as

$$
\mathbf{j}_{5, a}(\mathrm{OPE})=\mathbf{j}_{5, a}^{\mathrm{N} 3 \mathrm{LO}}(\mathrm{OPE})+\mathbf{j}_{5, a}^{\mathrm{N} 4 \mathrm{LO}}(\mathrm{OPE})
$$

and the two quantities on the r.h.s. are given by

$$
\begin{aligned}
\mathbf{j}_{5, a}^{\mathrm{N} 3 \mathrm{LO}}(\mathrm{OPE})= & \frac{g_{A}}{2 f_{\pi}^{2}}\left[2 c_{3} \tau_{2, a} \mathbf{k}_{2}+\left(\boldsymbol{\tau}_{1} \times \boldsymbol{\tau}_{2}\right)_{a}\left[\frac{i}{2 m} \mathbf{K}_{1}-\frac{c_{6}+1}{4 m} \boldsymbol{\sigma}_{1} \times \mathbf{q}\right.\right. \\
& \left.\left.+\left(c_{4}+\frac{1}{4 m}\right) \boldsymbol{\sigma}_{1} \times \mathbf{k}_{2}\right]\right] \boldsymbol{\sigma}_{2} \cdot \mathbf{k}_{2} \frac{1}{\omega_{2}^{2}}, \\
\mathbf{j}_{5, a}^{\mathrm{N} 4 \mathrm{LO}}(\mathrm{OPE})= & \frac{g_{A}^{5} m_{\pi}}{256 \pi f_{\pi}^{4}}\left[9 \tau_{2, a} \mathbf{k}_{2}-\left(\boldsymbol{\tau}_{1} \times \boldsymbol{\tau}_{2}\right)_{a} \boldsymbol{\sigma}_{1} \times \mathbf{k}_{2}\right] \boldsymbol{\sigma}_{2} \cdot \mathbf{k}_{2} \frac{1}{\omega_{2}^{2}} .
\end{aligned}
$$

The TPE axial charge, and MPE and short-range axial current, can be written, respectively, as

$$
\begin{aligned}
\rho_{5, a}^{\mathrm{N} 3 \mathrm{LO}}(\mathrm{TPE})= & i \frac{g_{A}^{3}}{128 \pi^{2} f_{\pi}^{4}}\left[\left(\boldsymbol{\tau}_{1} \times \boldsymbol{\tau}_{2}\right)_{a} \boldsymbol{\sigma}_{1} \cdot \mathbf{k}_{2}\left(3-\frac{1}{g_{A}^{2}}-\frac{4 m_{\pi}^{2}}{k_{2}^{2}+4 m_{\pi}^{2}}\right)-4 \tau_{1, a}\left(\boldsymbol{\sigma}_{1} \times \boldsymbol{\sigma}_{2}\right) \cdot \mathbf{k}_{2}\right] \\
& \times \frac{s_{2}}{k_{2}} \ln \left(\frac{s_{2}+k_{2}}{s_{2}-k_{2}}\right),
\end{aligned}
$$

with with $s_{j}$ defined as in Eq. (161), and

$$
\begin{aligned}
\widetilde{\mathbf{j}}_{5, a}^{\mathrm{N} 4 \mathrm{LO}}(\mathrm{MPE})= & \mathbf{j}_{5, a}^{\mathrm{N} 4 \mathrm{LO}}(\mathrm{MPE})-\frac{\mathbf{q}}{q^{2}+m_{\pi}^{2}} \mathbf{q} \cdot \mathbf{j}_{5, a}^{\mathrm{N} 4 \mathrm{LO}}(\mathrm{MPE}) \\
& +\frac{g_{A}^{3}}{128 \pi f_{\pi}^{4}} \frac{\mathbf{q}}{q^{2}+m_{\pi}^{2}}\left[\tau_{2, a}\left[Z_{1}\left(k_{1}\right) \boldsymbol{\sigma}_{2} \cdot\left(\mathbf{k}_{1}-\mathbf{k}_{2}\right)+Z_{2}\left(\mathbf{k}_{1}\right) \boldsymbol{\sigma}_{2} \cdot \mathbf{k}_{2} \frac{1}{\omega_{2}^{2}}\right]\right. \\
& +\left(2 \tau_{2, a}-\tau_{1, a}\right) Z_{1}\left(k_{2}\right) \boldsymbol{\sigma}_{1} \cdot \mathbf{k}_{2}+\left(\boldsymbol{\tau}_{1} \times \boldsymbol{\tau}_{2}\right)_{a}\left[Z _ { 3 } ( k _ { 1 } ) \left[\left(\boldsymbol{\sigma}_{1} \times \boldsymbol{\sigma}_{2}\right) \cdot \mathbf{k}_{1}\right.\right. \\
& \left.\left.\left.-2\left(\boldsymbol{\sigma}_{1} \times \mathbf{k}_{1}\right) \cdot\left(\mathbf{k}_{2}+\mathbf{q}\right) \boldsymbol{\sigma}_{2} \cdot \mathbf{k}_{2} \frac{1}{\omega_{2}^{2}}\right]+Z_{3}\left(k_{2}\right)\left(\boldsymbol{\sigma}_{1} \times \boldsymbol{\sigma}_{2}\right) \cdot \mathbf{k}_{2}\right]\right] \\
& +\frac{g_{A}^{3}}{128 \pi f_{\pi}^{4}} \tau_{2, a} Z_{1}\left(k_{1}\right)\left[\left(\mathbf{k}_{2}-3 \mathbf{k}_{1}\right) \boldsymbol{\sigma}_{2} \cdot \mathbf{k}_{2} \frac{1}{\omega_{2}^{2}}-2 \boldsymbol{\sigma}_{2}\right] \\
& +\frac{g_{A}^{3}}{32 \pi f_{\pi}^{4}}\left(\boldsymbol{\tau}_{1} \times \boldsymbol{\tau}_{2}\right)_{a} Z_{3}\left(k_{1}\right) \boldsymbol{\sigma}_{1} \times \mathbf{k}_{1} \boldsymbol{\sigma}_{2} \cdot \mathbf{k}_{2} \frac{1}{\omega_{2}^{2}}
\end{aligned}
$$

where

$$
\begin{aligned}
\mathbf{j}_{5, a}^{\mathrm{N} 4 \mathrm{LO}}(\mathrm{MPE})= & \frac{g_{A}^{3}}{64 \pi f_{\pi}^{4}} \tau_{2, a}\left[W_{1}\left(k_{2}\right) \boldsymbol{\sigma}_{1}+W_{2}\left(k_{2}\right) \mathbf{k}_{2} \boldsymbol{\sigma}_{1} \cdot \mathbf{k}_{2}\right] \\
& +\frac{g_{A}^{5}}{64 \pi f_{\pi}^{4}} \tau_{1, a} W_{3}\left(k_{2}\right)\left(\boldsymbol{\sigma}_{2} \times \mathbf{k}_{2}\right) \times \mathbf{k}_{2}
\end{aligned}
$$


and the loop functions $Z_{i}$ and $W_{i}$ are

$$
\begin{aligned}
W_{1}(k)= & \int_{0}^{1} d z\left[\left(1-5 g_{A}^{2}\right) M(k, z)-\frac{g_{A}^{2} k^{2}}{2}\left[\frac{9 z \bar{z}-1}{M(k, z)}-\frac{k^{2}(z \bar{z})^{2}}{M(k, z)^{3}}\right]\right] \\
W_{2}(k)= & \int_{0}^{1} d z\left[-\frac{g_{A}^{2}(z \bar{z})^{2} k^{2}}{2 M(k, z)^{3}}+\frac{z \bar{z}\left(7 g_{A}^{2}+2\right)-g_{A}^{2}}{2 M(k, z)}\right] \\
W_{3}(k)= & -\frac{1}{2} \int_{0}^{1} d z \frac{1}{M(k, z)}, \\
Z_{1}(k)= & \int_{0}^{1} d z\left[\frac{z \bar{z} k^{2}}{M(k, z)}+3 M(k, z)\right] \\
Z_{2}(\mathbf{k})= & \int_{0}^{1} d z\left[4 m_{\pi}^{3}-10 M(k, z)^{3}+M(k, z)\left(15 m_{\pi}^{2}+14 k^{2}-6 \mathbf{q} \cdot \mathbf{k}+6 q^{2}\right.\right. \\
& \left.\left.-20 z \bar{z} k^{2}\right)+\frac{z \bar{z} k^{2}}{M(k, z)}\left(5 m_{\pi}^{2}+4 k^{2}+2 q^{2}-2 \mathbf{q} \cdot \mathbf{k}-2 k^{2} z \bar{z}\right)\right] \\
Z_{3}(k)= & \int_{0}^{1} d z M(k, z),
\end{aligned}
$$

and $M(k, z)$ defined in Eq. (199).

\subsubsection{THREE-BODY CURRENTS}

We conclude the derivation by pointing out the following fact. In a three-nucleon system the two-body loop corrections to the axial current enter at order $Q^{-2}$, owing to the presence of a momentum-conserving $\delta$-function $\delta\left(\mathbf{p}_{3}^{\prime}-\mathbf{p}_{3}\right)$.

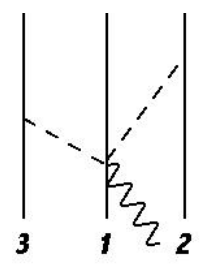

(a)

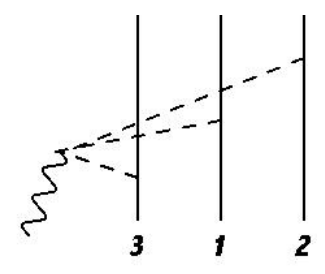

(b)

FIG. 15: Diagrams illustrating the three-body axial current at N4LO (i.e., order $Q^{-2}$ in a three-nucleon system). Nucleons, pions, and axial fields are denoted by solid, dashed, and wavy lines, respectively. Only a single time ordering is shown and pion-pole contributions are ignored.

These loop corrections turn out to be of the same order as the three-body axial current, 
illustrated in Fig. 23 and first derived in Ref. [48]. They are given by

$$
\begin{aligned}
\mathbf{j}_{5, a}^{\mathrm{N} 4 \mathrm{LO}}(3 \mathrm{~B})= & -\sum_{\mathrm{cyc}} \frac{g_{A}^{3}}{16 f_{\pi}^{4}}\left(2 \tau_{1, a} \boldsymbol{\tau}_{2} \cdot \boldsymbol{\tau}_{3}-\tau_{2, a} \boldsymbol{\tau}_{3} \cdot \boldsymbol{\tau}_{1}-\tau_{3, a} \boldsymbol{\tau}_{1} \cdot \boldsymbol{\tau}_{2}\right) \\
& \times\left(\boldsymbol{\sigma}_{1}-\frac{4}{3} \frac{\boldsymbol{\sigma}_{1} \cdot \mathbf{k}_{1} \mathbf{k}_{1}}{\omega_{1}^{2}}\right) \frac{\boldsymbol{\sigma}_{2} \cdot \mathbf{k}_{2}}{\omega_{2}^{2}} \frac{\boldsymbol{\sigma}_{3} \cdot \mathbf{k}_{3}}{\omega_{3}^{2}}
\end{aligned}
$$

where the sum is over the cyclic permutations of the three nucleons, and a momentumconserving $\delta$-function $(2 \pi)^{3} \delta\left(\mathbf{k}_{1}+\mathbf{k}_{2}+\mathbf{k}_{3}-\mathbf{q}\right)$ is implicit.

\subsection{CURRENT CONSERVATION IN THE CHIRAL LIMIT}

In the chiral limit $\left(m_{\pi} \rightarrow 0\right)$ the axial current is conserved and

$$
\mathbf{q} \cdot \mathbf{j}_{5, a}=\left[H, \rho_{5, a}\right]
$$

with the two-nucleon Hamiltonian given by (we ignore here three-nucleon potentials and currents)

$$
H=T^{(-1)}+v^{(0)}+v^{(2)}+\ldots
$$

where the superscripts denote the power counting, the $v^{(n)}$ are the two-nucleon potentials defined in Eqs. (86) and (88), and the kinetic energy $T^{(-1)}$ (in momentum space) is

$$
T^{(-1)}=\frac{\mathbf{p}_{1}^{2}}{2 m}(2 \pi)^{3} \delta\left(\mathbf{p}_{2}^{\prime}-\mathbf{p}_{2}\right)+(1 \rightleftharpoons 2)
$$

Here, the potentials and axial charge and current operators (including the axial coupling and pion decay constants and LECs entering them) are to be understood in the chiral limit. Order by order in the power counting, current conservation implies the following set of relations

$$
\begin{aligned}
\mathbf{q} \cdot \mathbf{j}_{5, a}^{(-3)} & =0 \\
\mathbf{q} \cdot \mathbf{j}_{5, a}^{(-1)} & =\left[T^{(-1)}, \rho_{5, a}^{(-2)}\right] \\
\mathbf{q} \cdot \mathbf{j}_{5, a}^{(0)} & =\left[T^{(-1)}, \rho_{5, a}^{(-1)}\right]+\left[v^{(0)}, \rho_{5, a}^{(-2)}\right], \\
\mathbf{q} \cdot \mathbf{j}_{5, a}^{(1)} & =\left[T^{(-1)}, \rho_{5, a}^{(0)}\right]+\left[v^{(0)}, \rho_{5, a}^{(-1)}\right],
\end{aligned}
$$

where we have only kept up to terms of order $Q^{2}$. Note that the commutators implicitly bring in factors of $Q^{3}$. The first of these relations is obviously satisfied, see Eqs. (147). The second relation has

$$
\mathbf{q} \cdot \mathbf{j}_{5, a}^{(-1)}=-\frac{g_{A}}{2 m^{2}} \tau_{1, a} \mathbf{k}_{1} \cdot \mathbf{K}_{1} \boldsymbol{\sigma}_{1} \cdot \mathbf{K}_{1}+(1 \rightleftharpoons 2)
$$


where $\mathbf{j}_{5, a}^{(-1)}$ is given by th N2LO contributions in Eq. (242), and it is also satisfied. The left-hand-side of the third relation has

$$
\mathbf{q} \cdot \mathbf{j}_{5, a}^{(0)}=i \frac{g_{A}}{4 m f_{\pi}^{2}}\left(\boldsymbol{\tau}_{1} \times \boldsymbol{\tau}_{2}\right)_{a} \boldsymbol{\sigma}_{2} \cdot \mathbf{k}_{2} \frac{1}{\omega_{2}^{2}}\left(\mathbf{k}_{1} \cdot \mathbf{K}_{1}+\mathbf{k}_{2} \cdot \mathbf{K}_{2}\right)+(1 \rightleftharpoons 2)
$$

and this matches the first commutator on the right-hand side, $\left[T^{(-1)}, \rho_{5, a}^{(-1)}\right]$ with $\rho_{5, a}^{(-1)}$ given by

$$
\rho_{5, a}^{(-1)}=i \frac{g_{A}}{4 f_{\pi}^{2}}\left(\boldsymbol{\tau}_{1} \times \boldsymbol{\tau}_{2}\right)_{a} \boldsymbol{\sigma}_{2} \cdot \mathbf{k}_{2} \frac{1}{\omega_{2}^{2}}+(1 \rightleftharpoons 2)
$$

i.e., the sum of terms a1 and a2 in Eqs. (155) and (156). There are additional contributions to $\mathbf{j}_{5, a}^{(0)}$, which arise from non-static corrections to the denominators involving pion energies in the diagrams illustrated in Fig. 16, where the crossed (full) circle means that these denominators are expanded as indicated in Eq. (82) to order $Q^{0}(Q)$ beyond the leading-order static term. These contributions have not been considered in this Thesis.
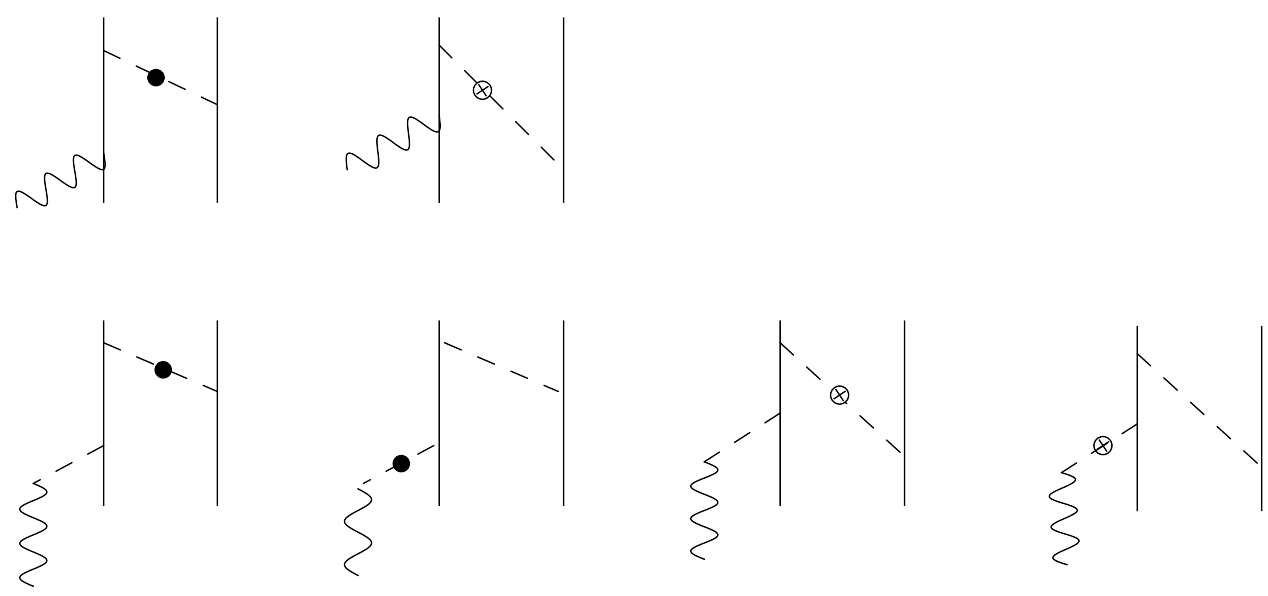

FIG. 16: Illustration of some of the non-static corrections to the axial current reported in Appendix H. Nucleons, pions, and axial fields are denoted by solid, dashed, and wavy lines, respectively. Pion lines with crossed (full) circle indicate that only the next-to-leading $Q^{0}$ (next-to-next-to-leading $Q$ ) term in the expansion of energy denominators, Eq. (82), are retained in the corresponding amplitudes.

Lastly, we consider the fourth relation, Eq. (265). The axial current $\mathbf{j}_{5, a}^{(1)}$ obtained in this 
Thesis is in the static limit, and one expects $\mathbf{q} \cdot \mathbf{j}_{5, a}^{(1)}$ to satisfy the commutator

$$
\begin{aligned}
{\left[v^{(0)}, \rho_{5, a}^{(-1)}\right]=-\frac{g_{A}^{3}}{16 f_{\pi}^{4}}\left(\tau_{1, a}-\tau_{2, a}\right) } & {\left[\left[k_{2}^{2} R^{(0)}\left(k_{2}\right)-R^{(2)}\left(k_{2}\right)\right] \boldsymbol{\sigma}_{1} \cdot \mathbf{k}_{2}\right.} \\
& \left.-\left[k_{1}^{2} R^{(0)}\left(k_{1}\right)-R^{(2)}\left(k_{1}\right)\right] \boldsymbol{\sigma}_{2} \cdot \mathbf{k}_{1}\right] \\
+\frac{g_{A}^{3}}{16 f_{\pi}^{4}}\left(\boldsymbol{\tau}_{1} \times \boldsymbol{\tau}_{2}\right)_{a} & {\left[R_{i j}^{(2)}\left(k_{2}\right) \sigma_{1, i}\left(\boldsymbol{\sigma}_{2} \times \mathbf{k}_{2}\right)_{j}\right.} \\
& \left.-R_{i j}^{(2)}\left(k_{1}\right) \sigma_{2, i}\left(\boldsymbol{\sigma}_{1} \times \mathbf{k}_{1}\right)_{j}\right]
\end{aligned}
$$

where the loop functions $R^{(n)}(k)$ and $R_{i j}^{(2)}(k)$ in the chiral limit read

$$
\begin{aligned}
& R^{(0)}(k) \rightarrow \frac{1}{16} \frac{1}{k}, \\
& R^{(2)}(k) \rightarrow-\frac{1}{16} k, \\
& R_{i j}^{(2)}(k) \rightarrow-\frac{1}{32} k \delta_{i j}+\ldots,
\end{aligned}
$$

and the ... indicate a term proportional to $k_{i} k_{j}$, which vanishes when inserted in Eq. (269). The current-conservation constraint is seen to be satisfied by noting that the only nonvanishing contributions to $\mathbf{q} \cdot \mathbf{j}_{5, a}^{(1)}$ are those due to diagrams e4, e5, e10, e15, e16, and e17 in Fig. 10, proportional to the combination of coupling constants $g_{A}^{3} / f_{\pi}^{4}$. In particular, the contributions of the purely irreducible diagrams e4, e5, e15, e16, and e17 combine to give

$$
\begin{gathered}
\mathbf{q} \cdot \mathbf{j}_{5, a}^{(1)}(\mathrm{e} 4+\mathrm{e} 5+\mathrm{e} 15+\mathrm{e} 16+\mathrm{e} 17)=-\frac{g_{A}^{3}}{32 f_{\pi}^{4}}\left[\tau_{1, a}\left[k_{2}^{2} R^{(0)}\left(k_{2}\right)-R^{(2)}\left(k_{2}\right)\right] \boldsymbol{\sigma}_{1} \cdot \mathbf{k}_{2}\right. \\
\left.+\tau_{2, a}\left[k_{1}^{2} R^{(0)}\left(k_{1}\right)-R^{(2)}\left(k_{1}\right)\right] \boldsymbol{\sigma}_{2} \cdot \mathbf{k}_{1}\right]+\frac{g_{A}^{3}}{32 f_{\pi}^{4}}\left(\boldsymbol{\tau}_{1} \times \boldsymbol{\tau}_{2}\right)_{a}\left[R_{i j}^{(2)}\left(k_{2}\right) \sigma_{1, i}\left(\boldsymbol{\sigma}_{2} \times \mathbf{k}_{2}\right)_{j}\right. \\
\left.-R_{i j}^{(2)}\left(k_{1}\right) \sigma_{2, i}\left(\boldsymbol{\sigma}_{1} \times \mathbf{k}_{1}\right)_{j}\right]
\end{gathered}
$$

with the remaining "missing" term being provided by $\mathbf{q} \cdot \mathbf{j}_{5, a}^{(1)}(\mathrm{e} 10)$. The other commutator $\left[T^{(-1)}, \rho_{5, a}^{(0)}\right]$ has a factor $1 / m$, and therefore non-static corrections need to be included in $\mathbf{j}_{5, a}^{(1)}$, if the latter is to satisfy the complete Eq. (265). These corrections have again been ignored in the present Thesis.

\subsection{COMPARISON WITH OTHER STUDIES}

We compare the one- and two-body axial charge and current operators derived here with those obtained by Park et al. in Refs. [28] and [48] in the heavy-baryon (HB) formulation of 
covariant perturbation theory and by Krebs et al. in Ref. [49], with the unitary transformation method. The one-body axial charge and current operators at leading order in Eqs. (147) and (148) are the same as those listed in Ref. [48], except for the pion-pole contribution to $\mathbf{j}_{5, a}^{(-3)}$, which, while nominally of the same order $\left(Q^{-3}\right)$ as the non-pole contribution, is nevertheless suppressed at low momentum transfer $q$ and is therefore ignored in Ref. [48] (we note incidentally that in that work $\mathbf{k}_{1}=-\mathbf{q}$, i.e., the opposite convention adopted here). Of course, this pion-pole contribution is crucial for current conservation in the chiral limit. We have neglected the $1 / m^{2}$ relativistic corrections to the leading order axial charge. These $1 / m^{2}$ corrections are retained in Ref. [48]. However, the $1 / m^{2}$ corrections to the leading order axial current in Eq. (152) are in agreement with those given in Ref. [48], except for the last term proportional to $\mathbf{q}\left(\boldsymbol{\sigma}_{1} \cdot \mathbf{q}\right)$, which was again ignored in that work. For the discussion of how the leading order axial charge emerges in covariant perturbation theory see Appendix J.

Turning to the OPE contributions at tree level, we find that the contributions to the axial charge $\rho_{5, a}^{(-1)}$ in Eq. (244) and axial current in Eq. 247 reproduce tose given in Ref. [28]. The contact terms contributing to the $Q^{0}$ axial current in Ref. [48] can be reduced through Fierz identities to the form given in Eq. (173).
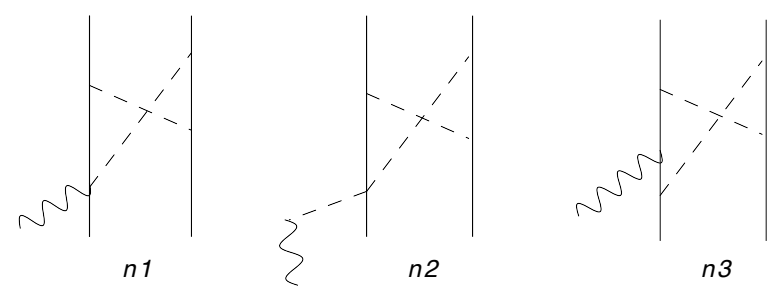

FIG. 17: Diagrams contributing to the axial charge (n1-n2) and current (n3) at order $Q$ considered in Ref. [28]. Nucleons, pions, and axial fields are denoted by solid, dashed, and wavy lines, respectively. Only a single time ordering is shown for each of the possible 12 (n1) and 60 (n2 and n3) cross-box topologies.

Next we consider loop corrections to the axial charge. The contributions of c3-c4, c7-c8, and c9-c12 in Fig. 8 are found to vanish in both approaches, here and in Refs. [28, 48]. The contributions of diagrams $\mathrm{c} 1$ and $\mathrm{c} 2$ are the same as in Ref. [28]. The contributions of diagrams c5 and c6 are different from those reported in Ref. [28] because of the different treatment of reducible topologies for these types of terms. Indeed, if only the (irreducible) cross-box topologies are retained for diagrams c5 and c6, as illustrated in Fig. 17, then the resulting operator is the same as those obtained in Ref. [48]. The OPE axial charge operator 
derived in Ref. [28] reads in our notation

$$
\begin{aligned}
& \rho_{5, a}(\mathrm{OPE}, \text { Park et al. })=i \frac{g_{A}}{4 f_{\pi}^{2}}\left(\boldsymbol{\tau}_{1} \times \boldsymbol{\tau}_{2}\right)_{a} \boldsymbol{\sigma}_{2} \cdot \mathbf{k}_{2} \frac{1}{\omega_{2}^{2}}\left[1-\frac{k_{2}^{2}}{f_{\pi}^{2}}\left(\frac{17 g_{A}^{2}+4}{144 \pi^{2}}+c_{3}^{r}\right)-\frac{m_{\pi}^{2} g_{A}^{2}}{12 \pi^{2} f_{\pi}^{2}}\right. \\
& \left.+\frac{g_{A}^{2}}{96 \pi^{2} f_{\pi}^{2}} \frac{s_{2}}{k_{2}} \ln \left(\frac{s_{2}+k_{2}}{s_{2}-k_{2}}\right)\left(5 k_{2}^{2}+8 m_{\pi}^{2}\right)+\frac{1}{96 \pi^{2} f_{\pi}^{2}}\left[\frac{s_{2}^{3}}{k_{2}} \ln \left(\frac{s_{2}+k_{2}}{s_{2}-k_{2}}\right)-8 m_{\pi}^{2}\right]\right] . \quad(274)
\end{aligned}
$$

Provided we define

$$
\widetilde{d}_{1}^{r}+\widetilde{d}_{2}^{r}-\widetilde{d}_{4}^{r}-\frac{\left(5+13 g_{A}^{2}\right)}{288}=-\frac{17 g_{A}^{2}+4}{144 \pi^{2} f_{\pi}^{2}}-c_{3}^{r},
$$

the expression above is in agreement with our Eq. (313) in the limit $\mathbf{q}=0$ (or $\mathbf{k}_{1}=-\mathbf{k}_{2}$ ) which is assumed in Refs. [28, 48], except for the term proportional to $m_{\pi}^{2}$ in the first line (note that $c_{3}^{r}$ is not to be confused with the LEC in $\mathcal{L}_{\pi N}^{(2)}$ ).

Moving on to the loop corrections to the axial current, the sum of the contributions due to diagram $\mathrm{m} 1$ of Fig. 14 and diagram e15 of Fig. 10 is the same as in Ref. [48], provided the parameter $\alpha$ in the $3 \pi A$ vertex of diagram e15 is set to $1 / 6$ - the authors of Refs. [28, 48] use the exponential parametrization for the pion field. The irreducible contributions of diagrams e1 and e4 in Fig. 10, and the contributions associated with the cross-box topologies of diagram e8 in Fig. 10 and illustrated in panel n3 of Fig. 17, are the same as those reported in Ref. [48].

Non-vanishing pion-pole diagrams e2, e5, e9, e10, e16, and e17 as well as diagrams e20e21 (e22-e25 vanish) in Fig. 10 have not been considered in Refs. [28, 48]. Because of this incomplete treatment, loop corrections to the axial current are $\alpha$-dependent in Refs. [28, 48]. Furthermore, the current is not conserved in the chiral limit.

In the following we compare our operators to those derived in Ref. [49]. That derivation has been done with the unitary transformation method, and it includes nonstatic corrections at order $Q$ which we have neglected. The formulas are rather involved and therefore we will limit ourselves to the comparison in the limit case $\mathbf{q}=0$. In this limit all pion-pole diagrams vanish, and the difference between the two expressions for the axial current is

$$
\begin{aligned}
\mathbf{j}_{5, a}^{\mathrm{TOPT}}(\mathbf{q}=0)-\mathbf{j}_{5, a}^{\mathrm{UT}}(\mathbf{q}=0)= & -\frac{g_{A}^{5}}{64 \pi f_{\pi}^{4}}\left[\tau_{1, a}\left(\boldsymbol{\sigma}_{2} \times \mathbf{k}_{2}\right) \times \mathbf{k}_{2} A\left(k_{2}\right)\right. \\
& +\tau_{2, a}\left[\boldsymbol{\sigma}_{1}\left[2 A\left(k_{2}\right) k_{2}^{2}+m_{\pi} \frac{10 m_{\pi}^{2}+3 k_{2}^{2}}{4 m_{\pi}^{2}+k_{2}^{2}}\right]\right. \\
& \left.\left.+\frac{\boldsymbol{\sigma}_{1} \cdot \mathbf{k}_{2} \mathbf{k}_{\mathbf{2}}}{k_{2}^{2}} A\left(k_{2}\right)\left(8 m_{\pi}^{2}+k_{2}^{2}\right)\right]\right],
\end{aligned}
$$

where $A\left(k_{2}\right)=1 /(2 k) \arctan \left[k /\left(2 m_{\pi}\right)\right]$. The factor $g_{A}^{5} / f_{\pi}^{4}$ in Eq. (275) indicates that the difference comes from the box diagram, see panel e8 of Fig. 10. In particular, we note that 
our prescription is linked to the off-the-energy-shell extension of the potential $\nu=0$. In particular we note that our operator was obtained by considering the $\nu=0$ prescription for the off-the-energy-shell extension of OPE and TPE potential. It is possible that differences in Eq. (275) might be due to the different off-the-energy-shell extrapolations adopted for these potentials.

For the axial charge operator expressions for two-pion exchange and short-range contributions agree. For loop corrections to the one-pion exchange there are differences in the logarithm free parts of Eq. (237). 


\section{CHAPTER 6}

\section{TRITIUM BETA DECAY}

In this chapter we carry out a calculation of the tritium $\beta$-decay rate and constrain the LEC $z_{0}$ in the axial current by reproducing the tritium Gamow-Teller (GT) matrix element. This decay has a $Q$-value of about $100 \mathrm{KeV}$ and therefore we evaluate current contributions at vanishing momentum transfer (i.e., all pion-pole contributions derived in the previous chapter are neglected). The LEC $z_{0}$ is related to the LEC $c_{D}$ entering the chiral three-nucleon potential at leading order [52] and therefore fixing $z_{0}$ is equivalent to fixing $c_{D}$. There is another LEC, denoted as $c_{E}$, which along with $c_{D}$ completely characterize the three-nucleon potential. In the past $c_{D}$ and $c_{E}$ have been fixed by reproducing the GT matrix element and the trinucleon binding energies $[54,55]$. Those calculations included the axial current up to order $Q^{0}$. Here we retain all contributions up to order $Q$.

\subsection{CONNECTION BETWEEN THE THREE-NUCLEON FORCE AND THE CONTACT AXIAL CURRENT}

The three-nucleon force at LO is given by the sum of the three diagrams in Fig. 18. Diagram a represents contact term,

$$
V_{\mathrm{a}}(i j k)=\sum_{\text {cyclic } i j k} \frac{c_{E}}{\Lambda_{\chi} f_{\pi}^{4}} \boldsymbol{\tau}_{i} \cdot \boldsymbol{\tau}_{k}
$$

while diagram $\mathrm{b}$ is the two-pion exchange (TPE) contibution

$$
V_{\mathrm{b}}(i j k)=\sum_{\text {cyclic } i j k} \frac{g_{A}^{2}}{4 f_{\pi}^{2}} \frac{1}{\omega_{1}^{2} \omega_{2}^{2}} \boldsymbol{\sigma}_{i} \cdot \mathbf{q}_{i} \tau_{i, a} F_{a c}(i j k) \tau_{k, c} \boldsymbol{\sigma}_{k} \cdot \mathbf{q}_{k},
$$

where

$$
F_{a c}(i j k)=-4 c_{1} \frac{m_{\pi}^{2}}{f_{\pi}^{2}} \delta_{a c}+\frac{2 c_{3}}{f_{\pi}^{2}} \mathbf{q}_{i} \cdot \mathbf{q}_{k} \delta_{a c}+\frac{c_{4}}{f_{\pi}^{2}} \boldsymbol{\sigma}_{j} \cdot\left(\mathbf{q}_{i} \times \mathbf{q}_{k}\right) \epsilon_{a c b} \tau_{j, b},
$$

and we have defined $\mathbf{q}_{i}=\mathbf{p}_{i}^{\prime}-\mathbf{p}_{i}$. Lastly diagram $c$ is the one-pion exchange term

$$
\widetilde{V}_{\mathrm{c}}(i j k)=-\sum_{\text {cyclic } i j k} \frac{g_{A}}{8 f_{\pi}^{2}} \frac{c_{D}}{\Lambda_{\chi} f_{\pi}^{2}}\left(\frac{\boldsymbol{\sigma}_{i} \cdot \mathbf{q}_{i} \boldsymbol{\sigma}_{j} \cdot \mathbf{q}_{j}}{\omega_{i}^{2}} \boldsymbol{\tau}_{i} \cdot \boldsymbol{\tau}_{j}+\frac{\boldsymbol{\sigma}_{j} \cdot \mathbf{q}_{k} \boldsymbol{\sigma}_{k} \cdot \mathbf{q}_{k}}{\omega_{k}^{2}} \boldsymbol{\tau}_{j} \cdot \boldsymbol{\tau}_{k}\right)
$$


where $c_{D}$ is the LEC multiplying the following contact interaction [12]

$$
\mathcal{L}_{4 N, u_{\mu}}^{(1)}=-\frac{c_{D}}{2 \Lambda_{\chi} f_{\pi}^{2}} \bar{N} \gamma^{\mu} \gamma_{5} u_{\mu} N \bar{N} N
$$

Since

$$
u_{\mu}=-\frac{1}{f_{\pi}} \boldsymbol{\tau} \cdot \partial_{\mu} \boldsymbol{\pi}+\boldsymbol{\tau} \cdot \mathbf{A}_{\mu}+\ldots,
$$

(see Appendix A), it is easy to see that the term proportional to the axial field leads precisely to the two-body contact axial current of Sec. 5.3. In particular it can be easily seen that the LEC $z_{0}$ of this contact term is linked to the LEC $c_{D}$ of the three-nucleon force through the following relation

$$
z_{0}=\frac{c_{D}}{2 \Lambda_{\chi} f_{\pi}^{2}}
$$
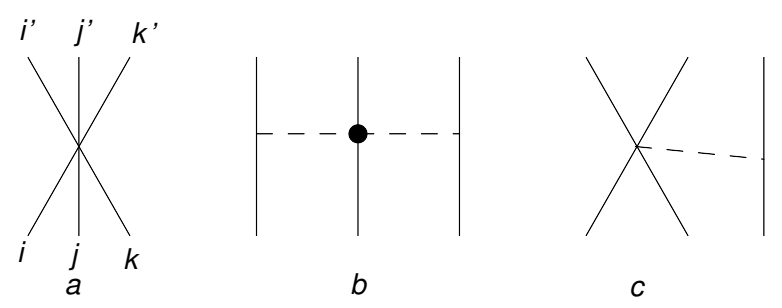

FIG. 18: Diagrams illustrating the three-nucleon force at leading order. Nucleons, pions, and axial fields are denoted by solid, dashed, and wavy lines, respectively. The full dot in diagrams b denotes corrections to the vertex coming from $\mathcal{L}_{\pi N}^{(2)}$. Only a single time ordering is shown for diagrams b and c. See text for further explanations.

In order to compare with previous determinations of this LEC [48, 54, 55], it is convenient to define the adimensional LEC $\hat{z}_{0}$ by rescaling $z_{0}$ as

$$
\hat{z}_{0}=\frac{2 m f_{\pi}^{2}}{g_{A}} z_{0}
$$

and the LEC $c_{D}$ in the three-nucleon potential related to $\hat{z}_{0}$ via $[48,54,55]$

$$
c_{D}=\frac{g_{A} \Lambda_{\chi}}{m} \hat{z}_{0}
$$

where $\Lambda_{\chi}$ is taken as $1 \mathrm{GeV}$ here (in Refs. $[54,55] \Lambda_{\chi}=0.7 \mathrm{GeV}$ was adopted). The scale $\Lambda_{\chi}$ is not to be confused with the cutoff $\Lambda$ which regularizes the configuration-space expressions of the axial operators. 


\subsection{NUCLEAR WEAK CURRENTS}

After defining the charge raising $(+)$ and lowering $(-)$ isospin operators as

$$
\tau_{i, \pm}=\frac{\tau_{i, x} \pm i \tau_{i, y}}{2}
$$

and

$$
\left(\boldsymbol{\tau}_{1} \times \boldsymbol{\tau}_{2}\right)_{ \pm}=\left(\boldsymbol{\tau}_{1} \times \boldsymbol{\tau}_{2}\right)_{x} \pm i\left(\boldsymbol{\tau}_{1} \times \boldsymbol{\tau}_{2}\right)_{ \pm}
$$

we introduce the charge changing axial current operators

$$
\mathbf{j}_{5, \pm}=\mathbf{j}_{5, x} \pm i \mathbf{j}_{5, y} .
$$

Referring to the summary in Sec. 5.5 we report in the following the one- and two-body nuclear axial currents in the limit of vanishing external field momentum. The one-body operator terms at LO and N2LO are

$$
\begin{aligned}
\mathbf{j}_{5, \pm}^{\mathrm{LO}} & =-g_{A} \tau_{1, \pm} \boldsymbol{\sigma}_{1} \\
\mathbf{j}_{5, \pm}^{\mathrm{N} 2 \mathrm{LO}} & =\frac{g_{A}}{2 m^{2}} \tau_{1, \pm}\left(K_{1}^{2} \boldsymbol{\sigma}_{1}-\mathbf{K}_{1} \boldsymbol{\sigma}_{1} \cdot \mathbf{K}_{1}\right)
\end{aligned}
$$

while the two-body operator at N3LO are separated into one-pion exchange (OPE) and contact (CT) terms corresponding respectively to Eqs. (173) and (245)

$$
\begin{aligned}
\mathbf{j}_{5, \pm}^{\mathrm{N} 3 \mathrm{LO}}(\mathrm{OPE})= & \frac{g_{A}}{2 f_{\pi}^{2}}\left\{4 c_{3} \tau_{2, \pm} \mathbf{k}+\left(\boldsymbol{\tau}_{1} \times \boldsymbol{\tau}_{2}\right)_{ \pm}\left[\left(c_{4}+\frac{1}{4 m}\right) \boldsymbol{\sigma}_{1} \times \mathbf{k}-\frac{i}{2 m} \mathbf{K}_{1}\right]\right\} \\
& \times \boldsymbol{\sigma}_{2} \cdot \mathbf{k} \frac{1}{\omega_{k}^{2}} \\
\mathbf{j}_{5, \pm}^{\mathrm{N} 3 \mathrm{LO}}(\mathrm{CT})= & z_{0}\left(\boldsymbol{\tau}_{1} \times \boldsymbol{\tau}_{2}\right)_{ \pm} \boldsymbol{\sigma}_{1} \times \boldsymbol{\sigma}_{2} .
\end{aligned}
$$

The (two-body) operator at N4LO are given by Eqs. (245) and (250),

$$
\begin{aligned}
\mathbf{j}_{5, \pm}^{\mathrm{N} 4 \mathrm{LO}}(\mathrm{OPE})= & \frac{g_{A}^{5} m_{\pi}}{256 \pi f_{\pi}^{4}}\left[18 \tau_{2, \pm} \mathbf{k}-\left(\boldsymbol{\tau}_{1} \times \boldsymbol{\tau}_{2}\right)_{ \pm} \boldsymbol{\sigma}_{1} \times \mathbf{k}\right] \boldsymbol{\sigma}_{2} \cdot \mathbf{k} \frac{1}{\omega_{k}^{2}} \\
\widetilde{\mathbf{j}}_{5, \pm}^{\mathrm{N} 4 \mathrm{LO}}(\mathrm{MPE})= & \frac{g_{A}^{3}}{32 \pi f_{\pi}^{4}} \tau_{2, \pm}\left[W_{1}(k) \boldsymbol{\sigma}_{1}+W_{2}(k) \mathbf{k} \boldsymbol{\sigma}_{1} \cdot \mathbf{k}+Z_{1}(k)\left(2 \mathbf{k} \boldsymbol{\sigma}_{2} \cdot \mathbf{k} \frac{1}{\omega_{k}^{2}}-\boldsymbol{\sigma}_{2}\right)\right] \\
& +\frac{g_{A}^{5}}{32 \pi f_{\pi}^{4}} \tau_{1, \pm} W_{3}(k)\left(\boldsymbol{\sigma}_{2} \times \mathbf{k}\right) \times \mathbf{k}-\frac{g_{A}^{3}}{32 \pi f_{\pi}^{4}}\left(\boldsymbol{\tau}_{1} \times \boldsymbol{\tau}_{2}\right)_{ \pm} Z_{3}(k) \boldsymbol{\sigma}_{1} \times \mathbf{k} \\
& \times \boldsymbol{\sigma}_{2} \cdot \mathbf{k} \frac{1}{\omega_{k}^{2}}
\end{aligned}
$$


where the loop functions are defined in Eqs. (252)-(257). Lastly, the expression of the three-nucleon axial current

$$
\begin{aligned}
\mathbf{j}_{5, \pm}^{\mathrm{N} 4 \mathrm{LO}}\left(3 \mathrm{~B} ; \mathbf{k}_{2}, \mathbf{k}_{3}\right)= & -\sum_{\mathrm{cyc}} \frac{g_{A}^{3}}{8 f_{\pi}^{4}}\left(2 \tau_{1, \pm} \boldsymbol{\tau}_{2} \cdot \boldsymbol{\tau}_{3}-\tau_{2, \pm} \boldsymbol{\tau}_{3} \cdot \boldsymbol{\tau}_{1}-\tau_{3, \pm} \boldsymbol{\tau}_{1} \cdot \boldsymbol{\tau}_{2}\right) \\
& \times \boldsymbol{\sigma}_{1} \frac{\boldsymbol{\sigma}_{2} \cdot \mathbf{k}_{2}}{\omega_{2}^{2}} \frac{\boldsymbol{\sigma}_{3} \cdot \mathbf{k}_{3}}{\omega_{3}^{2}}
\end{aligned}
$$

where the sum is over the cyclic permutations of the three nucleons, and in the $\mathbf{q}=0$ limit $\mathbf{k}_{1}=-\left(\mathbf{k}_{2}+\mathbf{k}_{3}\right)$. We have neglected the term proportional to $\boldsymbol{\sigma}_{1} \cdot \mathbf{k}_{1}$ in Eq. (294) since the computational effort required to numerically implement thi operator is unjustified in view of its expected contributions, see Table 1.

Configuration-space expressions for these two- and three-body operators (denoted generically as $2 \mathrm{~B}$ and $3 \mathrm{~B}$, respectively) follow from

$$
\begin{aligned}
\mathbf{j}_{5, \pm}(2 \mathrm{~B}) & =\int \frac{d \mathbf{k}}{(2 \pi)^{3}} \mathrm{e}^{i \mathbf{k} \cdot \mathbf{r}_{12}} C_{\Lambda}(k) \mathbf{j}_{5, \pm}(2 \mathrm{~B} ; \mathbf{k}), \\
\mathbf{j}_{5, \pm}(3 \mathrm{~B}) & =\int \frac{d \mathbf{k}_{2}}{(2 \pi)^{3}} \frac{d \mathbf{k}_{3}}{(2 \pi)^{3}} \mathrm{e}^{-i \mathbf{k}_{2} \cdot \mathbf{r}_{12}} \mathrm{e}^{-i \mathbf{k}_{3} \cdot \mathbf{r}_{13}} C_{\Lambda}\left(k_{2}\right) C_{\Lambda}\left(k_{3}\right) \mathbf{j}_{5, \pm}\left(3 \mathrm{~B} ; \mathbf{k}_{2}, \mathbf{k}_{3}\right),
\end{aligned}
$$

where the relative positions are defined as $\mathbf{r}_{i j}=\mathbf{r}_{i}-\mathbf{r}_{j}$, and $C_{\Lambda}(k)$ is the momentum cutoff, which we take as

$$
C_{\Lambda}(k)=\mathrm{e}^{-(k / \Lambda)^{4}} .
$$

This cutoff does not modify the power counting of the various terms, as it is easily seen by expanding in powers of $k / \Lambda$. Lastly, terms proportional to $\mathbf{K}_{j}$ in the N2LO and N3LO currents are obtained by replacing $\mathbf{K}_{j}$ with $-i \boldsymbol{\nabla}_{j}$ in configuration space (the momentum operator), and need to be symmetrized accordingly to preserve hermiticity. Explicit expressions for these Fourier transforms are listed in Appendix K.

The weak vector charge has in principle contributions from one- and two-body terms derived in Ref. [23]; however, the latter vanish in the limit $\mathbf{q}=0$, and only the one-body term at LO contributes in this limit $\rho_{ \pm}=\tau_{i, \pm}$ the well-known Fermi transition operator. 


\subsection{GAMOW-TELLER MATRIX ELEMENT IN TRITIUM $\beta$-DECAY}

The Gamow-Teller (GT) matrix element is obtained from the tritium half-life via (see [56] and references therein)

$$
\left(1+\delta_{R}\right) t f_{V}=\frac{K / G_{V}^{2}}{\langle\mathbf{F}\rangle^{2}+f_{A} / f_{V} g_{A}^{2}\langle\mathbf{G T}\rangle^{2}},
$$

where $g_{A}=1.2723$ is the current experimental value [57] for the nucleon axial coupling constant, $\delta_{R}=1.9 \%$ is the outer radiative correction [58], $t$ is the half-life of ${ }^{3} \mathrm{H}$, and $f_{V}$ and $f_{A}$ are Fermi functions reported in Ref. [59] to have the values $2.8355 \times 10^{-6}$ and $2.8505 \times 10^{-6}$, respectively. The experimental value used for $K / G_{V}^{2}$ is $(6144.5 \pm 1.9) \mathrm{s}$ as obtained in Ref. [60], and that used for $\left(1+\delta_{R}\right) t f_{V}$ is $(1134.6 \pm 3.1)$ s as reported in Ref. [59]. Finally, $\langle\mathbf{F}\rangle$ and $\langle\mathbf{G T}\rangle$ denote the reduced matrix element of the Fermi $(\mathrm{F})$ and GT operators

$$
\begin{aligned}
\langle\mathbf{F}\rangle & =\left\langle{ }^{3} \mathrm{He}\left\|\rho_{+}\right\|^{3} \mathrm{H}\right\rangle, \\
\langle\mathbf{G T}\rangle & =\left\langle{ }^{3} \mathrm{He}\left\|\mathbf{j}_{5,+}\right\|^{3} \mathrm{H}\right\rangle,
\end{aligned}
$$

where the $\left|{ }^{3} \mathrm{H}\right\rangle$ and $\left|{ }^{3} \mathrm{He}\right\rangle$ wave functions are obtained with the hyper spherical-harmonics (HH) expansion method (for a review see Ref. [61]) from two- and three-nucleon potentials derived from either $\chi \mathrm{EFT}$ or the phenomenological approach.

The combination of chiral potentials is denoted as N3LO/N2LO(500) [N3LO/N2LO(600)] corresponding to cutoff $\Lambda=500 \mathrm{MeV}(\Lambda=600 \mathrm{MeV})$, and consists of two-nucleon potentials at N3LO from Refs. $[9,62]$ and three-nucleon potentials at N2LO from Refs. [12, 63]. ${ }^{1}$ The combination of conventional potentials is denoted as AV18/UIX and consists of the Argonne $v_{18}$ (AV18) two-nucleon potential [64] and Urbana-IX (UIX) three-nucleon potential [65]. In all cases we obtain $\langle\mathbf{F}\rangle=0.9998$. From this value we extract via Eq. (298) the experimental GT matrix element as

$$
\mathrm{GT}_{\mathrm{EXP}}=\langle\mathbf{G T}\rangle_{\mathrm{EXP}} / \sqrt{3}=0.9511 \pm 0.0013
$$

Contributions to the GT matrix element corresponding to the LO, N2LO, N3LO, N4LO, and $\mathrm{N} 4 \mathrm{LO}(3 \mathrm{~B})$ axial operators are reported in Table 1 , where the LEC $z_{0}$ in the N3LO(CT) operator is taken as $z_{0}=1$ in units of $\mathrm{GeV}^{-3}$. The LECs $c_{3}$ and $c_{4}$ in the N3LO(OPE) operators are constrained by fits to $\pi N$ scattering data, and two different sets of values

\footnotetext{
${ }^{1}$ Note that for consistency with the convention adopted in Chapters 4 and 5 , it would be more appropriate to label these two- and three-nucleon potentials, respectively, as N4LO and N3LO. However, this is not the standard notation used in the literature.
} 
(listed in the table caption) have been used in the present study, one from Refs. [9, 62] and the other from a recent analysis of these data based on Roy-Steiner equations [66], specifically the values corresponding to the column labeled N3LO in Table II of that work. The first set of $c_{3}$ and $c_{4}$ values (from Refs. [9, 62]) enters the chiral two- and three-nucleon potentials, used here to generate the ${ }^{3} \mathrm{H}$ and ${ }^{3} \mathrm{He}$ wave functions. Clearly, use of the second set from Ref. [66] in the N3LO(OPE) axial current is not consistent with these potentials; results for the GT matrix element are provided in that case only to give an estimate of their sensitivity to the $c_{3}$ and $c_{4}$ values. As per the additional LECs $\left(c_{D}, c_{E}\right)$ in the three-nucleon potential, these have been obtained by the fitting procedure described below. In particular, we note that the LEC $z_{0}$ in the $\mathrm{N} 3 \mathrm{LO}(\mathrm{CT})$ operator is related to $c_{D}$ via Eq. (284). The GT (and F) matrix elements are computed exactly, without approximation, with quantum Monte Carlo methods. The spin-isospin algebra is carried out with techniques similar to those developed in Ref. [67] for the electromagnetic current operator. The results reported in the tables below are based on random walks consisting of $10^{6}$ configurations. Statistical errors are not listed, but are typically at the few parts in $10^{3}$, except in the special case of the N3LO(OPE) results, for which they are at the few \% level (see below).

In Table 1 we report the results for the N3LO/N2LO(500) and N3LO/N2LO(600) models, and in parentheses those for the AV18/UIX model. The LO and N2LO axial operators do not need to be regularized, and hence the corresponding contributions for the AV18/UIX are the same for $\Lambda=500 \mathrm{MeV}$ and $600 \mathrm{MeV}$. However, the N3LO/N2LO contributions change (rather significantly at N2LO) as $\Lambda$ varies in this range due to the intrinsic cutoff dependence of the potentials. In the N3LO axial current of Eq. (290) the terms proportional to $c_{3}$ and $c_{4}$ have opposite signs and tend to cancel each other. The degree of cancellation depends crucially on the values of the LECs and Hamiltonian model. In particular, when $c_{3}$ and $c_{4}$ are taken from Refs. [9, 62], the sum of their contributions for the N3LO/N2LO model is (in magnitude) comparable to the contribution from the non-local terms proportional to $\mathbf{K}_{i}$ in Eq. (290). 
TABLE 1: Contributions to the GT matrix element of tritium $\beta$-decay corresponding to the Hamiltonian model N3LO/N2LO (AV18/UIX) and cutoffs $\Lambda=500 \mathrm{MeV}$ and $600 \mathrm{MeV}$ in the chiral potentials and weak axial current operators. The acronyms LO, N2LO, N3LO(OPE), N3LO(CT), N4LO(OPE), N4LO(MPE), and N4LO(3B) refer, respectively, to the axial operators given in Eq. (288), Eq. (289), Eq. (290), Eq. (291), Eq. (292), Eq. (293), and Eq. (294). In the N3LO(OPE) operator the LECs $c_{3}$ and $c_{4}$ have the values $c_{3}=-3.20 \mathrm{GeV}^{-1}$ and $c_{4}=5.40 \mathrm{GeV}^{-1}$ from Refs. [9, 62], while in the $\mathrm{N}^{2} \mathrm{LO}^{\star}(\mathrm{OPE})$ operator they are taken as $c_{3}=-5.61 \mathrm{GeV}^{-1}$ and $c_{4}=4.26 \mathrm{GeV}^{-1}$ from Ref. [66]. The LEC $z_{0}$ in $\mathrm{N} 3 \mathrm{LO}(\mathrm{CT})$ is taken to have the value $z_{0}=1$ in units of $\mathrm{GeV}^{-3}$. The LECs $\left(c_{D}, c_{E}\right)$ in the three-nucleon chiral potential have the values $(-1.847,-0.548)$ for $\Lambda=500 \mathrm{MeV}$ and $(-2.030,-1.553)$ for $\Lambda=600 \mathrm{MeV}$. See text for further explanations.

\begin{tabular}{c|c|c}
$\Lambda$ & $500 \mathrm{MeV}$ & $600 \mathrm{MeV}$ \\
\hline \hline $\mathrm{LO}$ & $0.9363(0.9224)$ & $0.9322(0.9224)$ \\
\hline $\mathrm{N} 2 \mathrm{LO}$ & $-0.569(-0.844) \times 10^{-2}$ & $-0.457(-0.844) \times 10^{-2}$ \\
\hline $\mathrm{N} 3 \mathrm{LO}(\mathrm{OPE})$ & $0.825(1.304) \times 10^{-2}$ & $0.043(7.517) \times 10^{-2}$ \\
$\mathrm{~N}^{2} \mathrm{OO}^{\star}(\mathrm{OPE})$ & $0.579(0.812) \times 10^{-1}$ & $0.652(1.413) \times 10^{-1}$ \\
\hline $\mathrm{N} 3 \mathrm{LO}(\mathrm{CT})$ & $-0.586(-0.721) \times 10^{-3}$ & $-0.717(-0.644) \times 10^{-3}$ \\
\hline $\mathrm{N} 4 \mathrm{LO}(\mathrm{OPE})$ & $-0.697(-0.964) \times 10^{-2}$ & $-0.867(-1.216) \times 10^{-2}$ \\
\hline $\mathrm{N} 4 \mathrm{LO}(\mathrm{MPE})$ & $-0.430(-0.565) \times 10^{-1}$ & $-0.532(-0.775) \times 10^{-1}$ \\
\hline $\mathrm{N} 4 \mathrm{LO}(3 \mathrm{~B})$ & $-0.143(-0.183) \times 10^{-2}$ & $-0.153(-0.205) \times 10^{-2}$ \\
\hline
\end{tabular}

The contributions from loop corrections, row labeled MPE, are relatively large and comparable to those from OPE. As a matter of fact, when the values for the $c_{3}$ and $c_{4}$ LECs are from Refs. $[9,62]$, the N3LO(OPE) contributions are an order of magnitude smaller than the N4LO(MPE) in the case of the chiral potentials. The origin of this large contribution can be traced back to the term proportional to the loop function $W_{1}(k)$ in Eq. (293), specifically to the term with the factor $\left(1-5 g_{A}^{2}\right)$ in Eq. (252). It originates from box diagram e8 of Fig. 10. All the N4LO corrections have opposite signs relative to the LO and OPE at $Q^{0}$.

Next, we discuss the determination of the value for the LEC $z_{0}$ required to reproduce $\mathrm{GT}_{\mathrm{EXP}}$ for the various Hamiltonian models we consider, by retaining corrections in the axial current up to either N3LO or N4LO. Values for the LECs are reported in Table 2 for the hybrid calculation based on the AV18/UIX Hamiltonian model, and in Table 3 for the chiral Hamiltonian model. In Table 2 the values for $\hat{z}_{0}$ and $c_{D}$, related via Eq. (284) are listed, so that they can be compared with previous determinations $[48,55,68]$. These values are 
obtained by simply reproducing the central value of $\mathrm{GT}_{\mathrm{EXP}}$ in Eq. (301).

TABLE 2: Adimensional values of the LECs corresponding to the AV18/UIX Hamiltonian model and cutoffs $\Lambda=500 \mathrm{MeV}$ and $600 \mathrm{MeV}$ in the chiral axial current. The LEC $\hat{z}_{0}$ is determined by reproducing $\mathrm{GT}_{\mathrm{EXP}}$ in calculations including in this current corrections up to either N3LO or N4LO. The values for $\hat{z}_{0}$ and $c_{D}$ are obtained using the LECs $\left(c_{3}, c_{4}\right)=(-3.20,5.40)$ $\mathrm{GeV}^{-1}$ from Refs. [9, 62], those for $\hat{z}_{0}^{\star}$ and $c_{D}^{\star}$ using $\left(c_{3}, c_{4}\right)=(-5.61,4.26) \mathrm{GeV}^{-1}$ from Ref. [66], in both the N3LO and N4LO calculations.

\begin{tabular}{c|c|c||c|c} 
& \multicolumn{2}{|c||}{$\mathrm{N} 3 \mathrm{LO}$} & \multicolumn{2}{c}{$\mathrm{N} 4 \mathrm{LO}$} \\
\hline$\Lambda$ & 500 & 600 & 500 & 600 \\
\hline$\hat{z}_{0}$ & -0.421 & 0.742 & -1.607 & -1.048 \\
\hline$c_{D}$ & -0.571 & 1.007 & -2.180 & -1.421 \\
\hline \hline$\hat{z}_{0}^{\star}$ & 0.769 & 2.038 & -0.417 & 0.235 \\
\hline$c_{D}^{\star}$ & 1.043 & 2.764 & -0.566 & 0.318 \\
\hline
\end{tabular}

In order to determine the values corresponding to the chiral potentials, we proceed as in Ref. [55]. The ${ }^{3} \mathrm{H}$ and ${ }^{3} \mathrm{He}$ ground state wave functions are calculated using these potentials for $\Lambda=500 \mathrm{MeV}$ and $600 \mathrm{MeV}$. We span the range $c_{D} \in[-4,3]$, and, in correspondence to each $c_{D}$ in this range, determine $c_{E}$ so as to reproduce the binding energies of either ${ }^{3} \mathrm{H}$ or ${ }^{3} \mathrm{He}$. The resulting trajectories are essentially indistinguishable, as shown in Fig. 21 for $\Lambda=500 \mathrm{MeV}$ and in Fig. 22 for $\Lambda=600 \mathrm{MeV}$, and as already obtained in Ref. [55]. Then, for each set of $\left(c_{D}, c_{E}\right)$, the triton and ${ }^{3} \mathrm{He}$ wave functions are calculated and the GamowTeller matrix element, denoted as $\mathrm{GT}_{\mathrm{TH}}$, is determined, by including in the axial current corrections up to N3LO or N4LO. The ratio $\mathrm{GT}_{\mathrm{TH}} / \mathrm{GT}_{\mathrm{EXP}}$ for both values of the cutoff $\Lambda$ is shown in Fig. 19 for the N3LO case and Fig. 20 for the N4LO one. 


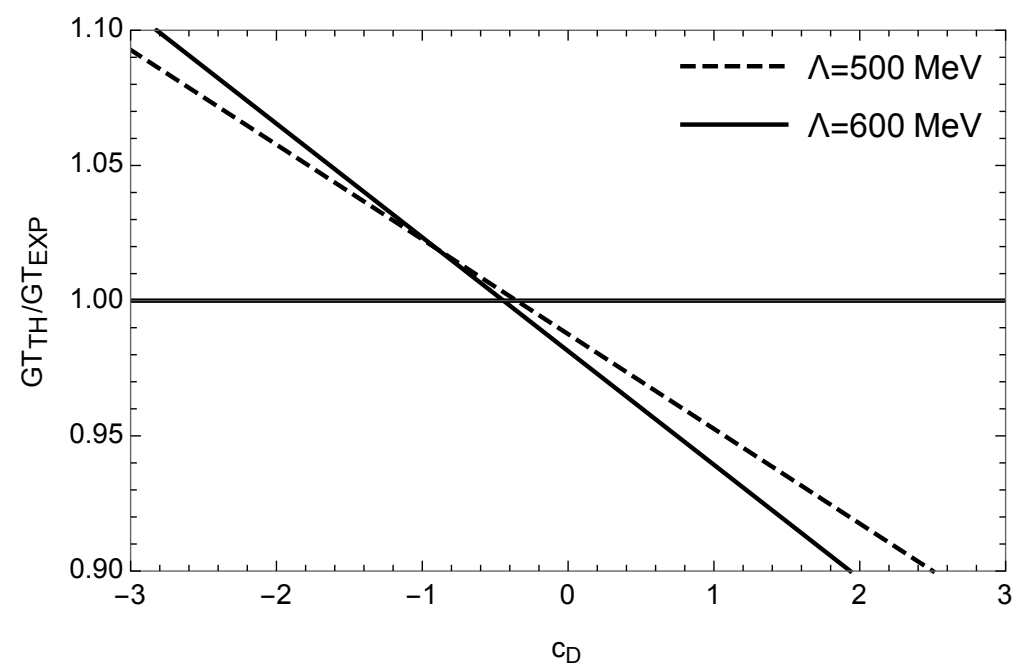

FIG. 19: The ratio $\mathrm{GT}_{\mathrm{TH}} / \mathrm{GT}_{\mathrm{EXP}}$ as function of the LEC $c_{D}$ obtained retaining corrections up to N3LO in the nuclear axial current. The results for both values of the cutoff $\Lambda$ are shown.

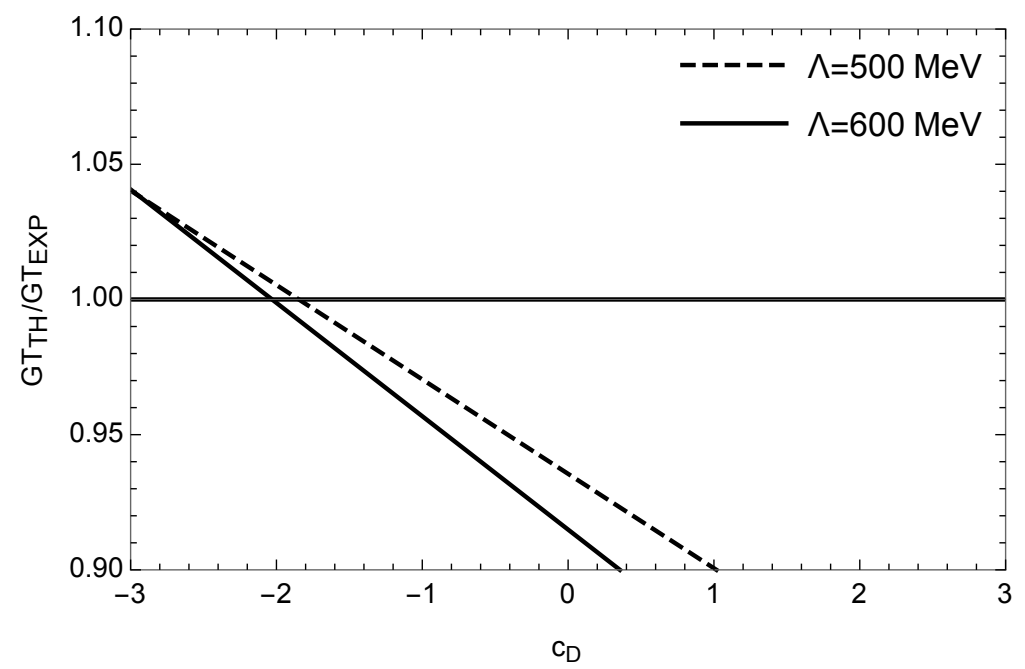

FIG. 20: Same as Fig. 19 but with the corrections in the axial current up to N4LO.

The LECs $\left(c_{D}, c_{E}\right)$ that reproduce simultaneously $\mathrm{GT}_{\mathrm{EXP}}$ (its central value) and the trinucleon binding energies are given in Table 3. 
TABLE 3: Values for the $\left(c_{D}, c_{E}\right)$ LECs as obtained by fitting the $A=3$ binding energy and $\mathrm{GT}_{\mathrm{EXP}}$ (its central value), using the N3LO/N2LO potential models with cutoffs $\Lambda=500$ $\mathrm{MeV}$ and $600 \mathrm{MeV}$. The results labelled N3LO and N4LO are obtained retaining in the nuclear axial current up to N3LO and N4LO contributions, respectively.

\begin{tabular}{c|c|c||c|c} 
& \multicolumn{2}{|c||}{$\mathrm{N} 3 \mathrm{LO}$} & \multicolumn{2}{c}{$\mathrm{N} 4 \mathrm{LO}$} \\
\hline$\Lambda$ & 500 & 600 & 500 & 600 \\
\hline$c_{D}$ & -0.353 & -0.443 & -1.847 & -2.030 \\
\hline$c_{E}$ & -0.305 & -1.224 & -0.548 & -1.553 \\
\hline \hline
\end{tabular}

The values for $c_{D}$ at $\mathrm{N} 3 \mathrm{LO}$ are found to be consistent with those listed in [55], after allowance is made for the different $\Lambda_{\chi}(0.7 \mathrm{GeV}$ in that work versus $1 \mathrm{GeV}$ above $)$ and for the fact that $\mathrm{GT}_{\mathrm{EXP}}$ as determined here is slightly smaller than adopted in [55].

Alternatively, we could choose a different set of three-nucleon observables to fit these LECs. We consider here, together with the $A=3$ binding energy, the $n d$ doublet scattering length $a_{n d}$, for which we take the experimental value $0.645 \pm 0.010 \mathrm{fm}$, obtained in Ref. [69]. In the range $c_{D} \in[-4,3]$ the resulting trajectories are displayed in Figs. 21 and 22 for $\Lambda=500 \mathrm{MeV}$ and $600 \mathrm{MeV}$, respectively. The experimental uncertainty in $a_{n d}$ has been taken into account, and therefore the results of Figs. 21 and 22 are presented as a band. 


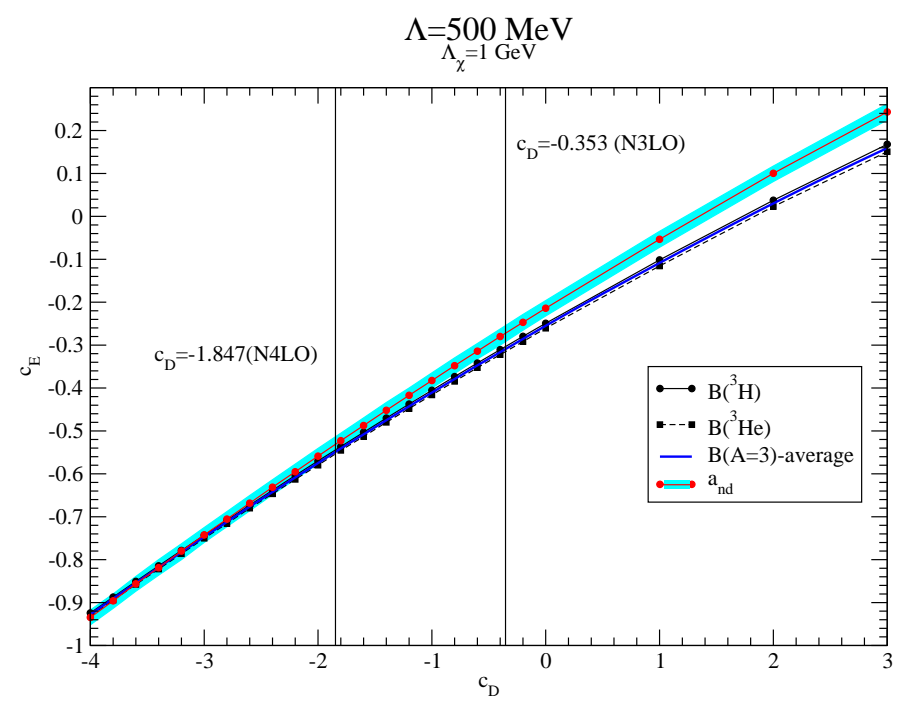

FIG. 21: The $c_{D}-c_{E}$ trajectories fitted to reproduce the experimental $A=3$ binding energies and the doublet $n d$ scattering length using the N3LO/N2LO potential with $\Lambda=500 \mathrm{MeV}$. The values of $8.475 \mathrm{MeV}, 7.725 \mathrm{MeV}$ and $0.645 \pm 0.010 \mathrm{fm}$ [69] are used for the ${ }^{3} \mathrm{H},{ }^{3} \mathrm{He}$ and $n d$ scattering length, respectively. Note that the $A=3$ binding energies have been corrected for the small contributions $\left(+7 \mathrm{keV}\right.$ in ${ }^{3} \mathrm{H}$ and $-7 \mathrm{keV}$ in $\left.{ }^{3} \mathrm{He}\right)$ due to the $n-p$ mass difference [70]. The (cyan) band is due to the experimental uncertainty on the $n d$ scattering length. The vertical lines indicate the $c_{D}$ values obtained by fitting $\mathrm{GT}_{\mathrm{EXP}}$ and retaining up to N4LO or N3LO contributions in the axial current. 


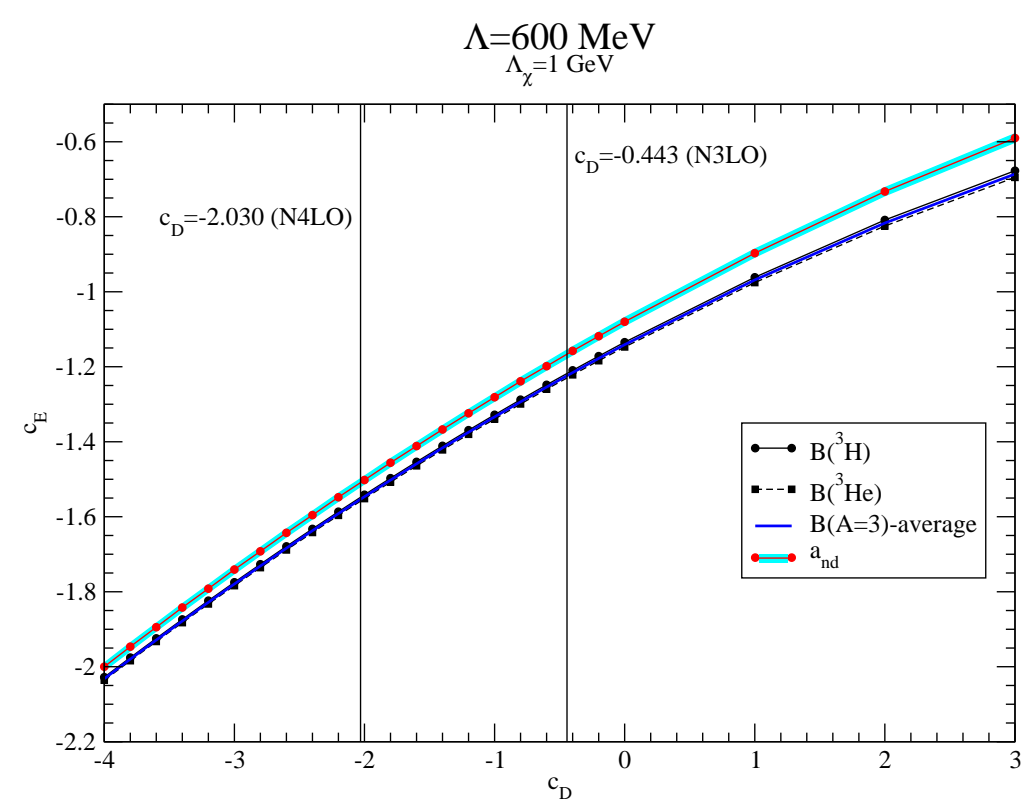

FIG. 22: Same as Fig. 21 but for $\Lambda=600 \mathrm{MeV}$.

The trajectories originating from the $A=3$ binding energies and $n d$ scattering length are quite close to each other, but do not overlap. In the $\Lambda=500 \mathrm{MeV}$ case, there is a crossing point at $\left(c_{D}, c_{E}\right)=(-2.340,-0.567)$, while for $\Lambda=600 \mathrm{MeV}$ there is no crossing. In particular, using the $\left(c_{D}, c_{E}\right)$ in Table 3 , we obtain $a_{n d}=0.654(0.665)$ fm for $\Lambda=500$ $\mathrm{MeV}$ and $a_{n d}=0.687(0.699)$ fm for $\Lambda=600 \mathrm{MeV}$, when the N4LO (N3LO) contributions in the axial current are retained. The present calculations of the $n d$ scattering wave functions ignore higher order electromagnetic interaction terms, such as those associated with the nucleons' magnetic moments. These terms are known to reduce the $a_{n d}$ value of about 3 $\%$ [61], when the AV18/UIX Hamiltonian model is used. Thus, the present analysis seems to indicate that the three $A=3$ observables ( $A=3$ binding energies, $\mathrm{GT}_{\mathrm{EXP}}$, and $\left.a_{n d}\right)$ are simultaneously reproduced, at least for $\Lambda=500 \mathrm{MeV}$, when the nuclear axial current retains corrections up to N4LO. 


\section{CHAPTER 7}

\section{INCLUSIVE NEUTRINO SCATTERING OFF THE}

\section{DEUTERON AT LOW ENERGIES}

In this chapter we discuss inclusive neutrino scattering off deuteron induced by neutral and charge-changing weak currents in a chiral EFT framework. A number of studies of these processes was carried out in the past several decades. These efforts culminated in a set of predictions [71, 72], in the early 2000's, for neutrino-deuteron cross sections for incoming neutrino energies up to $150 \mathrm{MeV}$. The calculations were based on the conventional mesonexchange framework, and used last-generation realistic potentials available at the time and a realistic model for the nuclear weak currents, which included one- and two-body terms. The Nakamura et al. studies played an important role in the analysis and interpretation of the Sudbury Neutrino Observatory (SNO) experiments [29], which have established solar neutrino oscillations and the validity of the standard model for the generation of energy and neutrinos in the sun [30].

Concurrent with those studies was a next-to-next-to-leading order calculation of neutrinodeuteron cross sections at low energies $(\lesssim 20 \mathrm{MeV})$ in an effective field theory in which pion degrees of freedom are integrated out and which is consequently parametrized in terms of contact terms [73]. In the strong-interaction sector, the LECs multiplying these contact terms were fixed by fitting the effective range expansions in the ${ }^{1} \mathrm{~S}_{0}$ and ${ }^{3} \mathrm{~S}_{1}$ two-nucleon channels (which dominate the low-energy cross sections). The weak current included onebody terms with couplings (nucleon magnetic moments and axial coupling constant) taken from experiment as well as two-body terms. In the vector sector, the two LECs associated with these two-body terms were determined by reproducing the radiative capture rate of neutrons on protons at thermal energies and the deuteron magnetic moment. In the axial sector the two-body terms were characterized by a single LEC (labeled $L_{1, A}$, and corresponding to our $z_{0}$ ), which however remained undetermined. Nevertheless, by fitting the results of Ref. [72], Butler and collaborators [73] were able to show that the resulting value for $L_{1, A}$ was natural, and that the calculated cross sections reproduced well the energy dependence obtained in the Nakamura et al. calculations. 
The energy range of the Nakamura et al. studies was extended up to $1 \mathrm{GeV}$ in the more recent calculations by Shen et al. [74]. These calculations too were based on the conventional framework, but included refinements in the modeling of the weak currents, which, however, turned out to have only a minor impact on the predicted cross sections [74]. The results confirmed those of Nakamura et al. in the energy range up to $150 \mathrm{MeV}$.

The calculation reported in this chapter differs from all previous ones in one essential aspect: it is fully based on a $\chi \mathrm{EFT}$ formulation of the nuclear potential $[9,62]$ and weak currents $[22,23,24,75,76]$ at high orders in the power counting. The LECs in the potential have been constrained by fitting the nucleon-nucleon scattering database in the energy range extending up to the pion-production threshold $[9,62]$, while the LECs in the electroweak current have been fitted to a number of low-energy electro-weak observables in the $A=2$ and 3 nuclei $[24,76]$ (specifically, the isoscalar and isovector magnetic moments of the deuteron and trinucleons, and the tritium Gamow-Teller matrix element).

A comparison among the results of the various calculations is discussed at the end of this chapter.

\subsection{NEUTRINO INCLUSIVE CROSS SECTION}

The differential cross section for neutrino $(\nu)$ and antineutrino $(\bar{\nu})$ inclusive scattering off a deuteron, specifically the processes ${ }^{2} \mathrm{H}\left(\nu_{l}, \nu_{l}\right) p n$ and ${ }^{2} \mathrm{H}\left(\bar{\nu}_{l}, \bar{\nu}_{l}\right) p n$ induced by neutral weak currents (NC) and denoted respectively as $\nu_{l}$-NC and $\bar{\nu}_{l}$-NC, and the processes ${ }^{2} \mathrm{H}\left(\nu_{e}, e^{-}\right) p p$ and ${ }^{2} \mathrm{H}\left(\bar{\nu}_{e}, e^{+}\right) n n$ induced by charge-changing weak currents (CC) and denoted respectively as $\nu_{l}$-CC and $\bar{\nu}_{l}$-CC, can be expressed as [74]

$$
\left(\frac{\mathrm{d} \sigma}{\mathrm{d} \epsilon^{\prime} \mathrm{d} \Omega}\right)_{\nu / \bar{\nu}}=\frac{G^{2}}{8 \pi^{2}} \frac{k^{\prime}}{\epsilon} F\left(Z, k^{\prime}\right)\left[v_{00} R_{00}+v_{z z} R_{z z}-v_{0 z} R_{0 z}+v_{x x} R_{x x} \mp v_{x y} R_{x y}\right],
$$

where $G=G_{F}$ for the NC processes and $G=G_{F} \cos \theta_{C}$ for the CC processes, and the - ( + ) sign in the last term is relative to the $\nu(\bar{\nu})$ initiated reactions. Following Ref. [72], we adopt the value $G_{F}=1.16637876 \times 10^{-5} \mathrm{GeV}^{-2}$ as obtained from an analysis of super-allowed $0^{+} \rightarrow 0^{+} \beta$-decays [60] - this value includes radiative corrections - while $\cos \theta_{C}$ is taken as 0.97425 from Ref. [77]. The initial neutrino four-momentum is $k^{\mu}=(\epsilon, \mathbf{k})$, the final lepton four momentum is $k^{\mu \prime}=\left(\epsilon^{\prime}, \mathbf{k}^{\prime}\right)$, and the lepton scattering angle is denoted by $\theta$. We have also defined the lepton energy and momentum transfers as $\omega=\epsilon-\epsilon^{\prime}$ and $\mathbf{q}=\mathbf{k}-\mathbf{k}^{\prime}$, respectively, and the squared four-momentum transfer as $Q^{2}=q^{2}-\omega^{2}>0$. The Fermi function $F\left(Z, k^{\prime}\right)$ with $Z=2$ accounts for the Coulomb distortion of the final lepton wave 
function in the $\mathrm{CC}$ reaction,

$$
F\left(Z, k^{\prime}\right)=2(1+\gamma)\left(2 k^{\prime} r_{d}\right)^{2 \gamma-2} \exp (\pi y)\left|\frac{\Gamma(\gamma+i y)}{\Gamma(1+2 \gamma)}\right|^{2}, \quad \gamma=\sqrt{1-(Z \alpha)^{2}}
$$

and it is set to one otherwise. Here $y=Z \alpha \epsilon^{\prime} / k^{\prime}, \Gamma(z)$ is the gamma function, $r_{d}$ is the deuteron charge radius $\left(r_{d}=1.97 \mathrm{fm}\right)$, and $\alpha$ is the fine structure constant. Radiative corrections for the $\mathrm{CC}$ and $\mathrm{NC}$ processes due to bremsstrahlung and virtual photon- and $Z$-exchanges have been evaluated by the authors of Refs. $[78,79]$ at the low energies $(\sim 10$ $\mathrm{MeV}$ ) most relevant for the SNO experiment, which measured the neutrino flux from the ${ }^{8} \mathrm{~B}$ decay in the sun. However, these corrections are not retained in the present work.

The factors $v_{\mu \nu}$ in Eq. (302) denote combinations of lepton kinematical variables including the final lepton mass, while the nuclear response functions are defined schematically as (explicit expressions for the $v_{\mu \nu}$ and $R_{\mu \nu}$ can be found in Ref. [74])

$$
R_{\mu \nu}(q, \omega) \sim \frac{1}{3} \sum_{M} \sum_{f} \delta\left(\omega+m_{d}-E_{f}\right)\left\langle f\left|j^{\mu}(\mathbf{q}, \omega)\right| d, M\right\rangle\left\langle f\left|j^{\nu}(\mathbf{q}, \omega)\right| d, M\right\rangle^{*}
$$

where $|d, M\rangle$ and $|f\rangle$ represent, respectively, the initial deuteron state in spin projection $M$ and the final two-nucleon state of energy $E_{f}$, and $m_{d}$ is the deuteron rest mass. The three-momentum transfer $\mathbf{q}$ is taken along the $z$-axis (i.e., the spin-quantization axis), and $j^{\mu}(\mathbf{q}, \omega)$ is the time component (for $\mu=0$ ) or space component (for $\mu=x, y, z$ ) of the $\mathrm{NC}$ or CC, denoted, respectively, by $j_{N C}^{\mu}$ or $j_{C C}^{\mu}$. The former is given by

$$
j_{N C}^{\mu}=-2 \sin ^{2} \theta_{W} j_{\gamma, S}^{\mu}+\left(1-2 \sin ^{2} \theta_{W}\right) j_{\gamma, z}^{\mu}+j_{5, z}^{\mu},
$$

where $\theta_{W}$ is the Weinberg angle $\left(\sin ^{2} \theta_{W}=0.2312[77]\right), j_{\gamma, S}^{\mu}$ and $j_{\gamma, z}^{\mu}$ include, respectively, the isoscalar and isovector terms of the electromagnetic current, and $j_{5, z}^{\mu}$ includes the isovector terms of the axial current (the subscript $z$ on these indicates that they transform as the $z$-component of an isovector under rotations in isospin space).

The charge-changing weak current reads

$$
j_{C C}^{\mu}=j_{ \pm}^{\mu}+j_{5, \pm}^{\mu}
$$

where $j_{ \pm}^{\mu}$ and $j_{5, \pm}^{\mu}$ are respectively the weak vector and weak axial currents defined as

$$
j_{ \pm}^{\mu}=j_{x}^{\mu} \pm i j_{y}^{\mu}, \quad j_{5, \pm}^{\mu}=j_{5, x}^{\mu} \pm i j_{5, y}^{\mu} .
$$

We observe that the conserved-vector-current hypothesis relates $j_{ \pm}^{\mu}$ to the isovector component $j_{\gamma, z}^{\mu}$ of the electromagnetic current via

$$
\left[\tau_{1, a}+\tau_{2, a}, j_{\gamma, z}^{\mu}\right]=2 i \epsilon_{a z b} j_{b}^{\mu}
$$


Before turning to a brief discussion of the one- and two-body $\chi$ EFT contributions to the NC and $\mathrm{CC}$, we note that, as described in considerable detail in Ref. [74], we evaluate, by direct numerical integrations, the matrix elements of the weak current between the deuteron and the two-nucleon scattering states labeled by the relative momentum $\mathbf{p}$ and in given pair-spin and pair-isospin channels, thus avoiding cumbersome multipole expansions. Differential cross sections are then obtained by integrating over $\mathbf{p}$ and summing over the discrete quantum numbers the appropriate matrix-element combinations entering the response functions [74]. 


\subsection{ELECTRO-WEAK CURRENT}

The $\chi$ EFT contributions up to one loop to the electromagnetic current $\left(\mathbf{j}_{\gamma}\right)[22,24]$ and charge $\left(\rho_{\gamma}\right)[23,24]$ are illustrated diagrammatically in Figs. 23 and 24, while those to the weak axial current $\left(\mathbf{j}_{5, a}\right)$ and charge $\left(\rho_{5, a}\right)$, obtained in this Thesis (see also Refs. [75, 76]) in Figs. 25 and 26. We recall here that the former are denoted below as $\mathbf{j}_{\gamma}=j_{\gamma}^{i}$ and $\rho_{\gamma}=j_{\gamma}^{0}$, and the latter as $\mathbf{j}_{5}=j_{5, z}^{i}$ and $\rho_{5}=j_{5, z}^{0}$, respectively, and subscripts specifying isospin components are dropped for simplicity here.
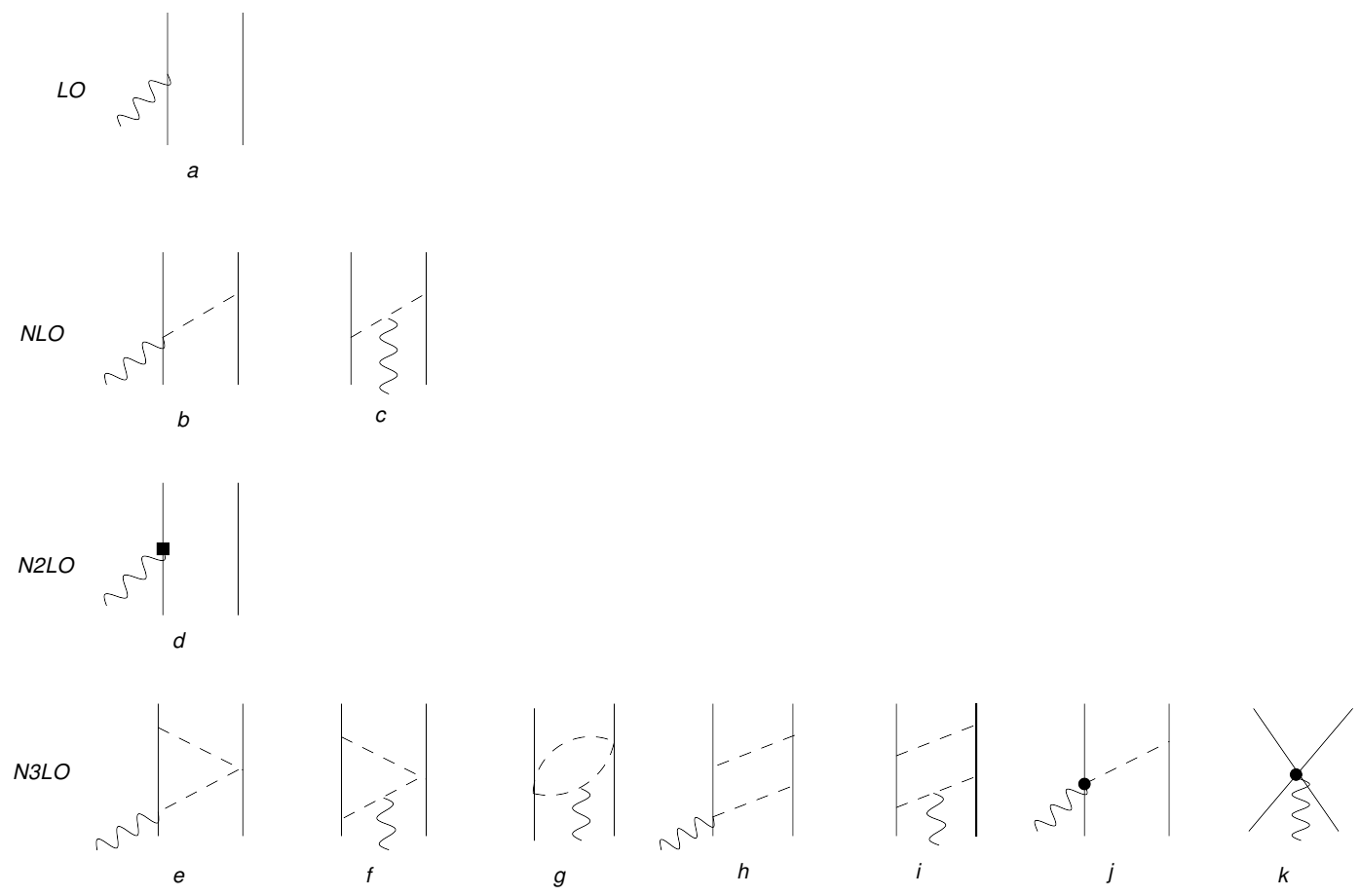

FIG. 23: Diagrams illustrating one- and two-body electromagnetic currents entering at $Q^{-2}$ (LO), $Q^{-1}(\mathrm{NLO}), Q^{0}(\mathrm{~N} 2 \mathrm{LO})$, and $Q^{1}$ (N3LO). Nucleons, pions, and photons are denoted by solid, dashed, and wavy lines, respectively. The square in panel $(\mathrm{d})$ represents the $(Q / m)^{2}$ relativistic correction to the LO one-body current ( $m$ is the nucleon mass); the solid circle in panel ( $\mathrm{j}$ ) is associated with the $\gamma \pi N$ coupling involving the LECs $d_{8}, d_{9}$, and $2 d_{21}-d_{22}$ in the $\pi N$ chiral Lagrangian $\mathcal{L}_{\pi N}^{(3)}[41]$; the solid circle in panel (k) denotes two-body contact terms of minimal and non-minimal nature, the latter involving two unknown LECs (see text). Only one among all possible time orderings is shown for the NLO and N3LO currents, so that all direct- and crossed-box contributions are accounted for.

The electromagnetic currents from LO, NLO, and N2LO terms and from N3LO loop corrections depend only on the nucleon axial coupling $g_{A}$ and and pion decay amplitude 
$f_{\pi}$ (NLO and N3LO), and the nucleon magnetic moments (LO and N2LO). Unknown LECs enter the N3LO OPE contribution involving the $\gamma \pi N$ vertex from the chiral Lagrangian $\mathcal{L}_{\pi N}^{(3)}$ (see Ref. [41]) as well as the contact currents implied by non-minimal couplings, as discussed in Sec. 7.2.1. On the other hand, in the charge operator there are no unknown LECs up to one loop, and OPE contributions, illustrated in panels (c)-(e) of Fig. 24, only appear at N3LO.
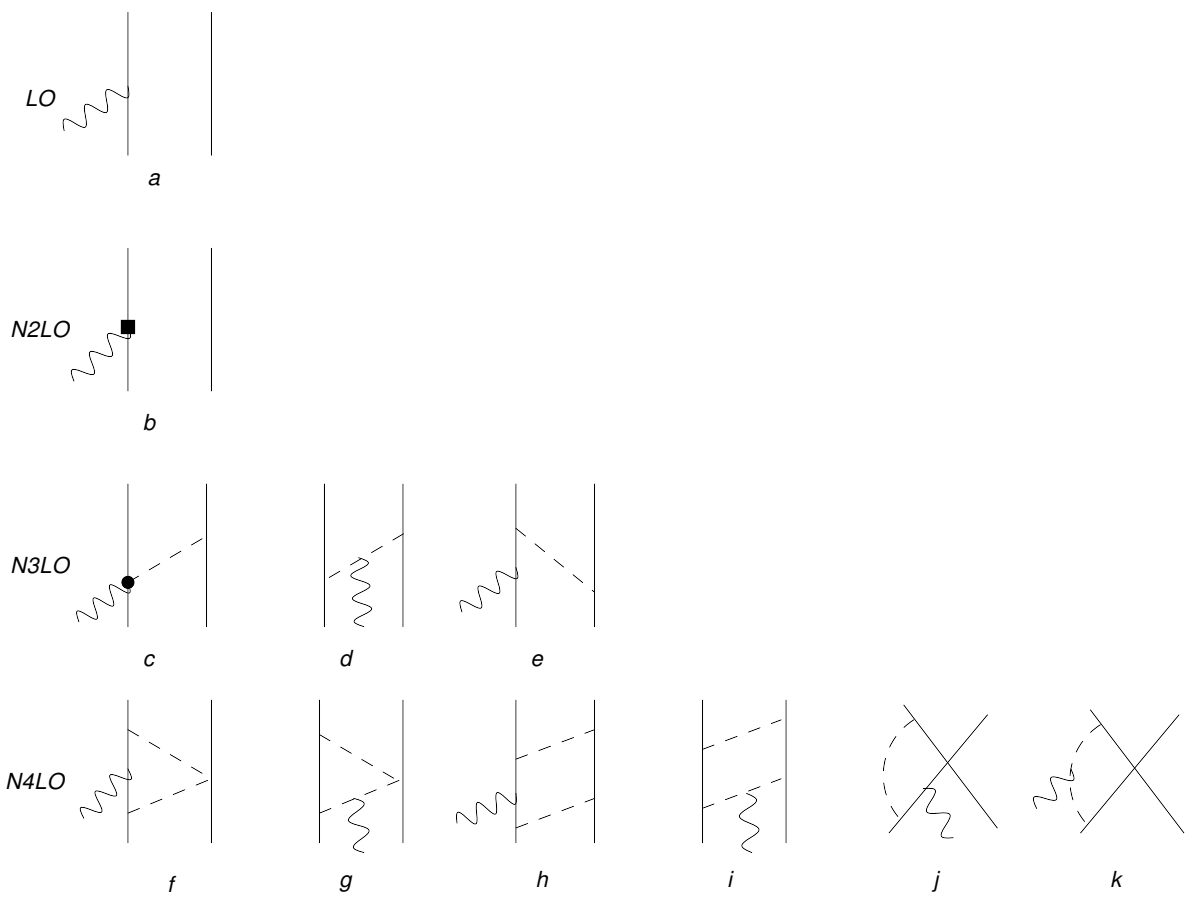

FIG. 24: Diagrams illustrating one- and two-body electromagnetic charge operators entering at $Q^{-3}$ (LO), $Q^{-1}$ (N2LO), $Q^{0}$ (N3LO), $Q^{1}$ (N4LO). The square in panel (b) represents the $(Q / m)^{2}$ relativistic correction to the LO one-body charge operator, whereas panel (c) represents the charge operator $\rho_{\gamma}^{(0)}(\mathrm{OPE})$ given in Eq. (309). As in Fig. 23, only a single time ordering is shown for the N3LO and N4LO contributions.

The contributions in panels (d) and (e) involve non-static corrections [23], while those in panel (c) were first derived by Phillips [80]

$$
\rho_{\gamma}^{(0)}(\mathrm{OPE})=\frac{g_{A}^{2}}{8 m f_{\pi}^{2}}\left(\boldsymbol{\tau}_{1} \cdot \boldsymbol{\tau}_{2}+\tau_{2 z}\right) \frac{\boldsymbol{\sigma}_{1} \cdot \mathbf{q} \boldsymbol{\sigma}_{2} \cdot \mathbf{k}_{2}}{k_{2}^{2}+m_{\pi}^{2}}
$$

This operator plays an important role in yielding predictions for the $A=2-4$ charge form factors that are in excellent agreement with the experimental data at low and moderate values of the momentum transfer $(q \lesssim 1 \mathrm{GeV} / \mathrm{c})[18,24]$. The calculations in Ref. [24] also 
showed that the non-static corrections of pion range from panels (d) and (e) of Fig. 24 are typically an order of magnitude smaller than those generated by panel (c).
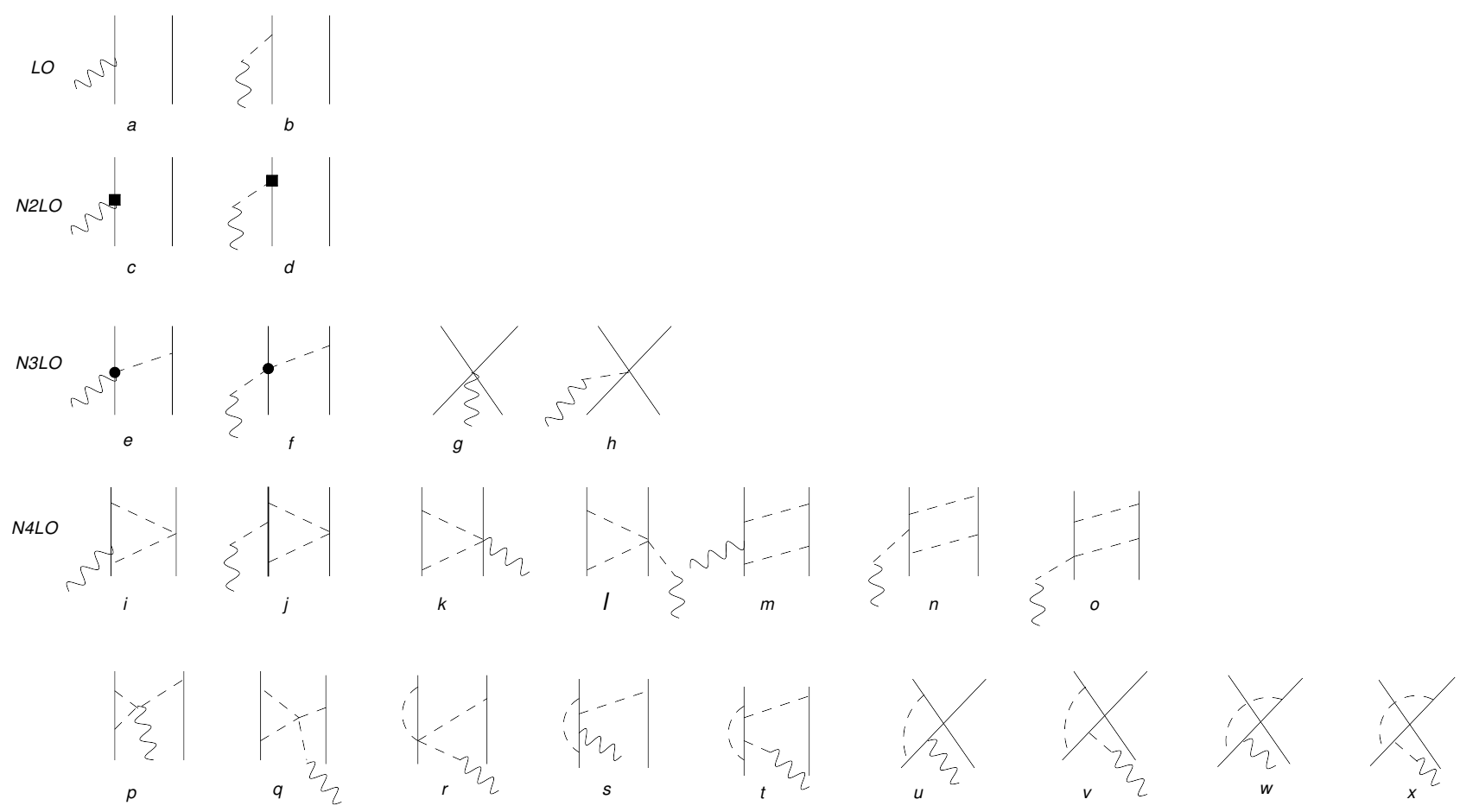

FIG. 25: Diagrams illustrating one- and two-body axial currents entering at $Q^{-3}$ (LO), $Q^{-1}$ (N2LO), $Q^{0}$ (N3LO), and $Q^{1}$ (N4LO). Nucleons, pions, and axial fields are denoted by solid, dashed, and wavy lines, respectively. The squares in panels (c) and (d) denote relativistic corrections to the one-body axial current, while the circles in panels (e) and (f) represent vertices implied by the $\mathcal{L}_{\pi N}^{(2)}$ chiral Lagrangian [41], involving the LECs $c_{i}$ (see Ref. [75] for additional explanations). As in Fig. 23, only a single time ordering is shown.

The axial current and charge operators illustrated in Figs. 25 and 26 include pion-pole contributions, which turned out to be relevant for the current to be conserved in the chiral limit as we saw in Sec. 5.6. In the electromagnetic current the divergent parts of the loop integrals are reabsorbed by the LECs multiplying contact terms [22], while those in the electromagnetic charge cancel out, in line with the fact that there are no counter-terms at N4LO [23]. We recall here that in the case of the axial operators, there are no divergencies in the current, while those in the charge lead to renormalization of the LECs multiplying contact-type contributions. In particular, the infinities in loop corrections to the OPE axial charge (not shown in Fig. 26) are re-absorbed by renormalization of the LECs $d_{i}$ in the $\mathcal{L}_{\pi N}^{(3)}$ chiral Lagrangian. 

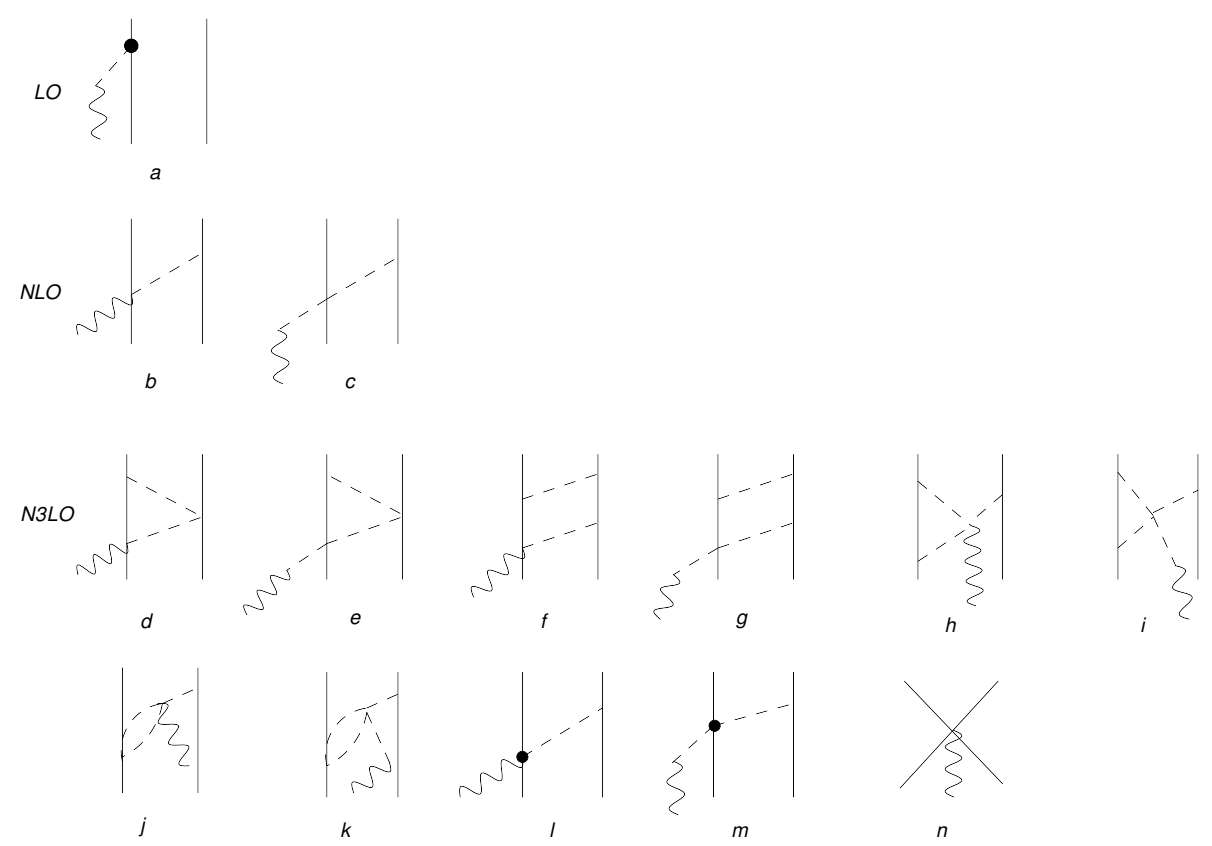

FIG. 26: Diagrams illustrating one- and two-body axial charge operators entering at $Q^{-2}$ (LO), $Q^{-1}$ (N1LO), and $Q^{1}$ (N3LO). Nucleons, pions, and axial fields are denoted by solid, dashed, and wavy lines, respectively. The diamonds in panels $(\mathrm{l})$ and $(\mathrm{m})$ indicate higher order $A \pi N$ vertices implied by the $\mathcal{L}_{\pi N}^{(3)}$ chiral Lagrangian [41], involving the LECs $d_{i}$ (see Ref. [75] for additional explanations). As in Fig. 23, only a single time ordering is shown.

\subsubsection{CONSTRAINING THE LECS IN THE ELECTRO-WEAK CURRENTS}

There is a total of ten LECs entering the two-body electro-weak currents discussed above, five of these are in the electromagnetic (vector) sector and the remaining five (in the limit of vanishing momentum transfer) in the axial sector. In the vector sector, contact terms originate from minimal and non-minimal couplings. The LECs multiplying the former are known from fits of the two-nucleon scattering database [24]. Non minimal couplings enter through the electromagnetic field tensor, and it has been shown [22] that only two independent structures occur at order $Q$ (see panel (k) in Fig. 23):

$$
\mathbf{j}_{\gamma}^{(1)}(\mathrm{CT})=-i e\left[\widetilde{c}_{\gamma}^{S} \boldsymbol{\sigma}_{1}+\widetilde{c}_{\gamma}^{V}\left(\tau_{1, z}-\tau_{2, z}\right) \boldsymbol{\sigma}_{1}\right] \times \mathbf{q},
$$

where $e$ is the electric charge, $\widetilde{c}_{\gamma}^{S}$ and $\widetilde{c}_{\gamma}^{V}$ are the two LECs, and the superscripts specify the isoscalar $(S)$ and isovector $(V)$ character of the associated operator. There is also a pion-range two-body operator resulting from sub-leading $\gamma \pi N$ couplings associated with the 
$\mathcal{L}_{\pi N}^{(3)}$ Lagrangian, and illustrated by panel (j) in Fig. 23. It reads:

$$
\begin{aligned}
\mathbf{j}_{\gamma}^{(1)}(\mathrm{OPE})= & i e \frac{g_{A}}{4 f_{\pi}^{2}} \frac{\boldsymbol{\sigma}_{2} \cdot \mathbf{k}_{2}}{k_{2}^{2}+m_{\pi}^{2}}\left[\left(\widetilde{d}_{\gamma, 1}^{V} \tau_{2, z}+\widetilde{d}_{\gamma}^{S} \boldsymbol{\tau}_{1} \cdot \boldsymbol{\tau}_{2}\right) \mathbf{k}_{2}\right. \\
& \left.-\widetilde{d}_{\gamma, 2}^{V}\left(\boldsymbol{\tau}_{1} \times \boldsymbol{\tau}_{2}\right)_{z} \boldsymbol{\sigma}_{1} \times \mathbf{k}_{2}\right] \times \mathbf{q}
\end{aligned}
$$

where the LECs $\widetilde{d}_{\gamma, 1}^{V}, \widetilde{d}_{\gamma, 2}^{V}$ and $\widetilde{d}_{\gamma}^{S}$ are related [24] to the LECs $d_{8}, d_{9}, d_{21}$, and $d_{22}$ in the original $\mathcal{L}_{\pi N}^{(3)}$ Lagrangian [41] in the following way

$$
\widetilde{d}_{\gamma}^{S}=-8 d_{9}, \quad \widetilde{d}_{\gamma, 1}^{V}=-8 d_{8}, \quad \widetilde{d}_{\gamma, 2}^{V}=2 d_{21}-d_{22} .
$$

As discussed below, these LECs have been determined by a combination of resonance saturation arguments and fits to photo-nuclear data in the two- and three-nucleon systems.

In the weak axial sector, the single contact term at order $Q^{0}$ (or N3LO, see panels (g) and (h) of Fig. 25) has one LEC $z_{0}$ and it has been fixed in the previous chapter. The axial charge operators at N3LO from OPE [panels (l) and (m) of Fig. 26] and contact interactions [panel (n)] involve, in principle, nine LECs [75]. Since the processes of interest in the present work are relatively low-momentum transfer ones, however, we have considered here these operators in the limit $q \rightarrow 0$ (or $\mathbf{k}_{1} \simeq-\mathbf{k}_{2}$ ), which leads to

$$
\begin{aligned}
\rho_{5, a}^{(1)}(\mathrm{OPE}) & =i \frac{g_{A}}{384 \pi^{2} f_{\pi}^{4}}\left(\boldsymbol{\tau}_{1} \times \boldsymbol{\tau}_{2}\right)_{a}\left\{g_{A}^{2}\left[\left(5 k_{2}^{2}+8 m_{\pi}^{2}\right) \frac{s_{2}}{k_{2}} \ln \frac{s_{2}+k_{2}}{s_{2}-k_{2}}-\frac{13}{3} k_{2}^{2}+2 m_{\pi}^{2}\right]\right. \\
& \left.+\left(\frac{s_{2}^{3}}{k_{2}} \ln \frac{s_{2}+k_{2}}{s_{2}-k_{2}}-\frac{5}{3} k_{2}^{2}-8 m_{\pi}^{2}\right)+\widetilde{d}_{5,1}^{V} k_{2}^{2}+\widetilde{d}_{5,2}^{V} m_{\pi}^{2}\right\} \frac{\boldsymbol{\sigma}_{2} \cdot \mathbf{k}_{2}}{k_{2}^{2}+m_{\pi}^{2}} \\
\rho_{5, a}^{(1)}(\mathrm{CT}) & =i \widetilde{c}_{5,2}^{V}\left(\boldsymbol{\tau}_{1} \times \boldsymbol{\tau}_{2}\right)_{a} \boldsymbol{\sigma}_{1} \cdot \mathbf{k}_{1}+i \widetilde{c}_{5,3}^{V} \tau_{1, a}\left(\boldsymbol{\sigma}_{1} \times \boldsymbol{\sigma}_{2}\right) \cdot \mathbf{k}_{2}+(1 \rightleftharpoons 2)
\end{aligned}
$$

where we recall that $s_{j}=\sqrt{k_{j}^{2}+4 m_{\pi}^{2}}$. The LECs $\widetilde{d}_{5, i}^{V}$ denote the combinations [75]

$$
\widetilde{d}_{5,1}^{V}=4\left(d_{1}+d_{2}+d_{3}\right), \quad \widetilde{d}_{5,2}^{V}=4\left(d_{1}+d_{2}+d_{3}\right)+8 d_{5},
$$

in terms of the $d_{i}$ 's in $\mathcal{L}_{\pi N}^{(3)}$ [41], and are taken from an analysis of $\pi N$ scattering data as reported in Ref. [62]. The LECs $\widetilde{c}_{5, i}^{V}$ where $\widetilde{c}_{5,2}^{V}=z_{2}$ and $\widetilde{c}_{5,3}^{V}=z_{3}$ have yet to be determined.

TABLE 4: The LECs in units of powers of $1 / \Lambda$ ( $\Lambda$ is the short-range cutoff) as in Eq. (317). Their values are adimensional. See text for forther explanations.

\begin{tabular}{c|ccc|cc||cc|ccc}
\hline$\Lambda(\mathrm{MeV})$ & $d_{\gamma}^{S}$ & $d_{\gamma, 1}^{V}$ & $d_{\gamma, 2}^{V}$ & $c_{\gamma}^{S}$ & $c_{\gamma}^{V}$ & $d_{5,1}^{V}$ & $d_{5,2}^{V}$ & $c_{5,1}^{V}$ & $c_{5,2}^{V}$ & $c_{5,3}^{V}$ \\
\hline 500 & 0.219 & 3.458 & 0.865 & 4.072 & -7.981 & -0.210 & 0.690 & 13.22 & 0.062 & 0.062 \\
600 & 0.323 & 4.980 & 1.245 & 11.38 & -11.69 & -0.302 & 0.994 & 25.07 & 0.130 & 0.130 \\
\hline
\end{tabular}


The two-body operators are regularized with the same short-range cutoff $C_{\Lambda}(k)=$ $\exp \left(-k^{4} / \Lambda^{4}\right)$ used in the previous chapter. In the electromagnetic sector, the two isoscalar LECs $\widetilde{c}_{\gamma}^{S}$ and $\widetilde{d}_{\gamma}^{S}$ are fixed (for each $\Lambda$ ) by reproducing the deuteron and isoscalar trinucleon magnetic moments, while the two isovector LECs $\widetilde{d}_{\gamma, 1}^{V}$ and $\widetilde{d}_{\gamma, 2}^{V}$ are constrained by assuming $\Delta$-resonance saturation [24],

$$
\widetilde{d}_{\gamma, 1}^{V}=\frac{4 \mu_{\gamma N \Delta} h_{A}}{9 m\left(m_{\Delta}-m\right)}, \quad \widetilde{d}_{\gamma, 2}^{V}=\frac{1}{4} \widetilde{d}_{\gamma, 1}^{V},
$$

where $m_{\Delta}-m=294 \mathrm{MeV}, h_{A} /\left(2 f_{\pi}\right)=f_{\pi N \Delta} / m_{\pi}$ with $f_{\pi N \Delta}^{2} /(4 \pi)=0.35$ as obtained by equating the first-order expression of the $\Delta$-decay width to the experimental value, and the transition magnetic moment $\mu_{\gamma N \Delta}$ is taken as $3 \mu_{N}$ [81]. The remaining LEC $\widetilde{c}_{\gamma}^{V}$ is determined by reproducing the isovector trinucleon magnetic moment [24]. In the weak axial sector, the LEC $\widetilde{c}_{5,1}^{V}$ is fixed by reproducing the tritium Gamow-Teller matrix element [76], while the other two LECs $\widetilde{c}_{5,2}^{V}$ and $\widetilde{c}_{5,3}^{V}$ in the axial charge are taken here to assume natural values $\widetilde{c}_{5, i}^{V} \simeq 1 / \Lambda_{\chi}^{4}$, for $i=2,3$ and with $\Lambda_{\chi}=1 \mathrm{GeV}$. However, cross sections results are insensitive to variations of $\widetilde{c}_{5,2}^{V}$ and $\widetilde{c}_{5,3}^{V}$ over a rather broad range (see Sec. 7.3). In Table 4 we list the values of all these LECs in units of the short-range cutoff $\Lambda$, namely

$$
\begin{aligned}
& \widetilde{d}_{\gamma}^{S}=d_{\gamma}^{S} / \Lambda^{2}, \quad \widetilde{d}_{\gamma, i}^{V}=d_{\gamma, i}^{V} / \Lambda^{2}, \quad \widetilde{c}_{\gamma}^{S}=c_{\gamma}^{S} / \Lambda^{4}, \quad \widetilde{c}_{\gamma}^{V}=c_{\gamma}^{V} / \Lambda^{4}, \\
& \widetilde{d}_{5, i}^{V}=d_{5, i}^{V} / \Lambda^{2}, \quad \widetilde{c}_{5,1}^{V}=c_{5,1}^{V} / \Lambda^{3}, \quad \widetilde{c}_{5,2}^{V}=c_{5,2}^{V} / \Lambda^{4}, \quad \widetilde{c}_{5,3}^{V}=c_{5,3}^{V} / \Lambda^{4} \text {. }
\end{aligned}
$$

Finally, we note that, since the processes under consideration involve small but nonvanishing four-momentum transfers $Q^{2}$, hadronic electro-weak form factors need to be included in the $\chi \mathrm{EFT}$ operators. Some of these form factors have been calculated in chiral perturbation theory [82], but the convergence of this calculation in powers of the momentum transfer appears to be rather poor. For this reason, in the results reported below, the form factors in the electromagnetic current and charge are accounted for as in Ref. [24], i.e., the nucleon, pion, and $N \Delta$-transition electromagnetic form factors are taken from fits to available electron scattering data. For the case of the axial charge and current, the operators are simply multiplied by $G_{A}\left(Q^{2}\right) / g_{A}$, where $G_{A}\left(Q^{2}\right)$ is the nucleon axial form factor, parametrized as $G_{A}\left(Q^{2}\right)=g_{A} /\left(1+Q^{2} / \Lambda_{A}^{2}\right)^{2}$ with $\Lambda_{A}=1 \mathrm{GeV}$, consistently with available neutrino scattering data (see [74] and references therein).

\subsection{CROSS-SECTION PREDICTIONS}

Total cross sections, integrated over the final lepton energy and scattering angle and obtained for the $\nu_{e}$-CC, $\bar{\nu}_{e}-\mathrm{CC}, \nu_{l}-\mathrm{NC}$, and $\bar{\nu}_{l}$-NC processes, are shown, respectively, in 
Figs. 27-30, where they are compared to the corresponding predictions from Ref. [72] for incoming neutrino energies ranging from threshold up to $150 \mathrm{MeV}$.

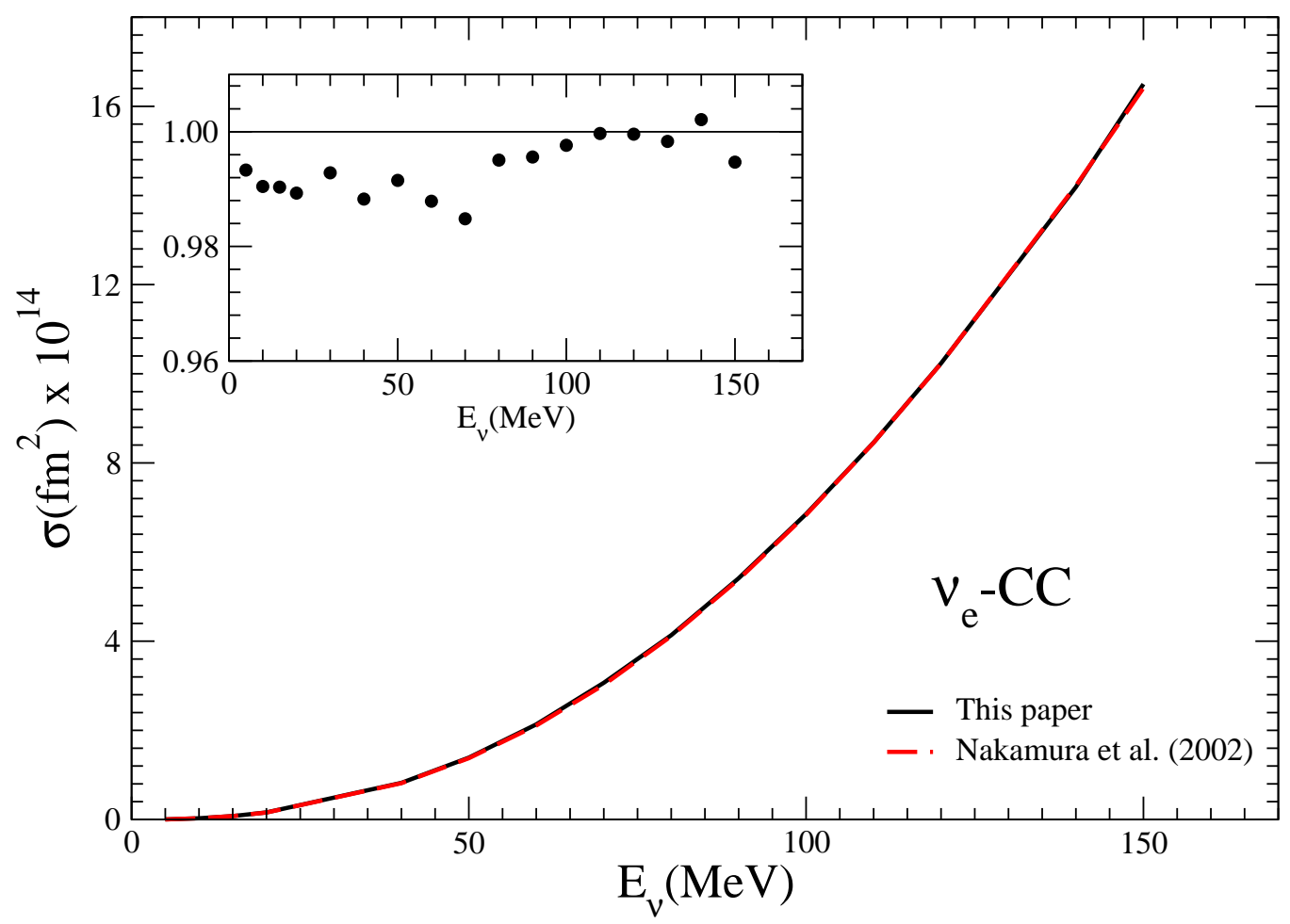

FIG. 27: (Color online) Total cross sections in $\mathrm{fm}^{2}$ for the $\nu_{e}$-CC induced process on the deuteron. The solid line corresponds to the $\chi$ EFT calculation with cutoff $\Lambda=500 \mathrm{MeV}$, based on the chiral potential of Ref. [9] and including electro-weak contributions up to N3LO in the vector current and axial charge, and up to N4LO in the axial current and vector charge, see Figs. 23-26. The dashed line is obtained within the conventional meson-exchange picture of Ref. [72]. The inset shows the ratio of conventional to $\chi$ EFT predictions. 


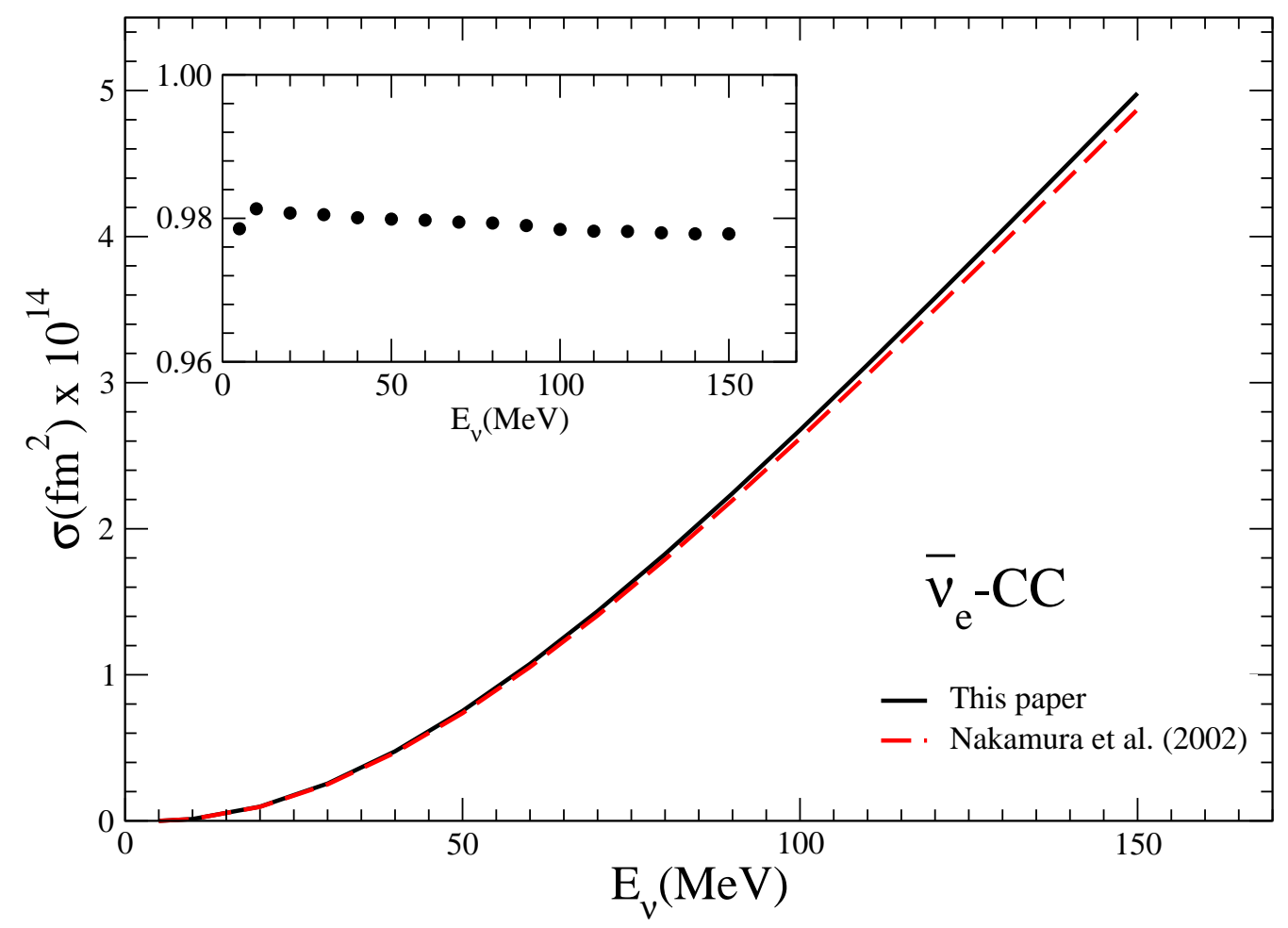

FIG. 28: (Color online) Same as in Fig. 27 but for the $\bar{\nu}_{e}$-CC induced process on the deuteron.

The present $\chi$ EFT calculations are based on the Entem and Machleidt potentials of Refs. [9, 62] corresponding to cutoffs $\Lambda=500$ and $600 \mathrm{MeV}$, and weak (vector and axial) current and charge operators of Refs. [22, 23, 24, 75], as described in the previous section. Matrix elements of these operators, suitably regularized as in Sec. 7.2.1, between the initial deuteron and final two-nucleon scattering states are evaluated with the methods developed in Ref. [74]. In practice, this entails obtaining the two-nucleon radial wave functions from solutions of the Lippmann-Schwinger equation in pair spin-isospin ST channels with total angular momentum $J \leq J_{\max }$, and in approximating these radial wave functions by spherical Bessel functions in channels with $J>J_{\max }$. The full wave function, labeled by the relative momentum $\mathbf{p}$ (and corresponding energy $p^{2} /(2 \mu), \mu$ being the reduced mass) and the discrete quantum numbers $S T$, is then reconstructed from its partial-wave expansion [74]. Consequently, interaction (including Coulomb in the case of two protons) effects in the final scattering states are exactly accounted for only in channels with $J \leq J_{\max }$. For the neutrino 
energies of interest here, however, we find that these effects are negligible when $J_{\max } \gtrsim 5$ [74].

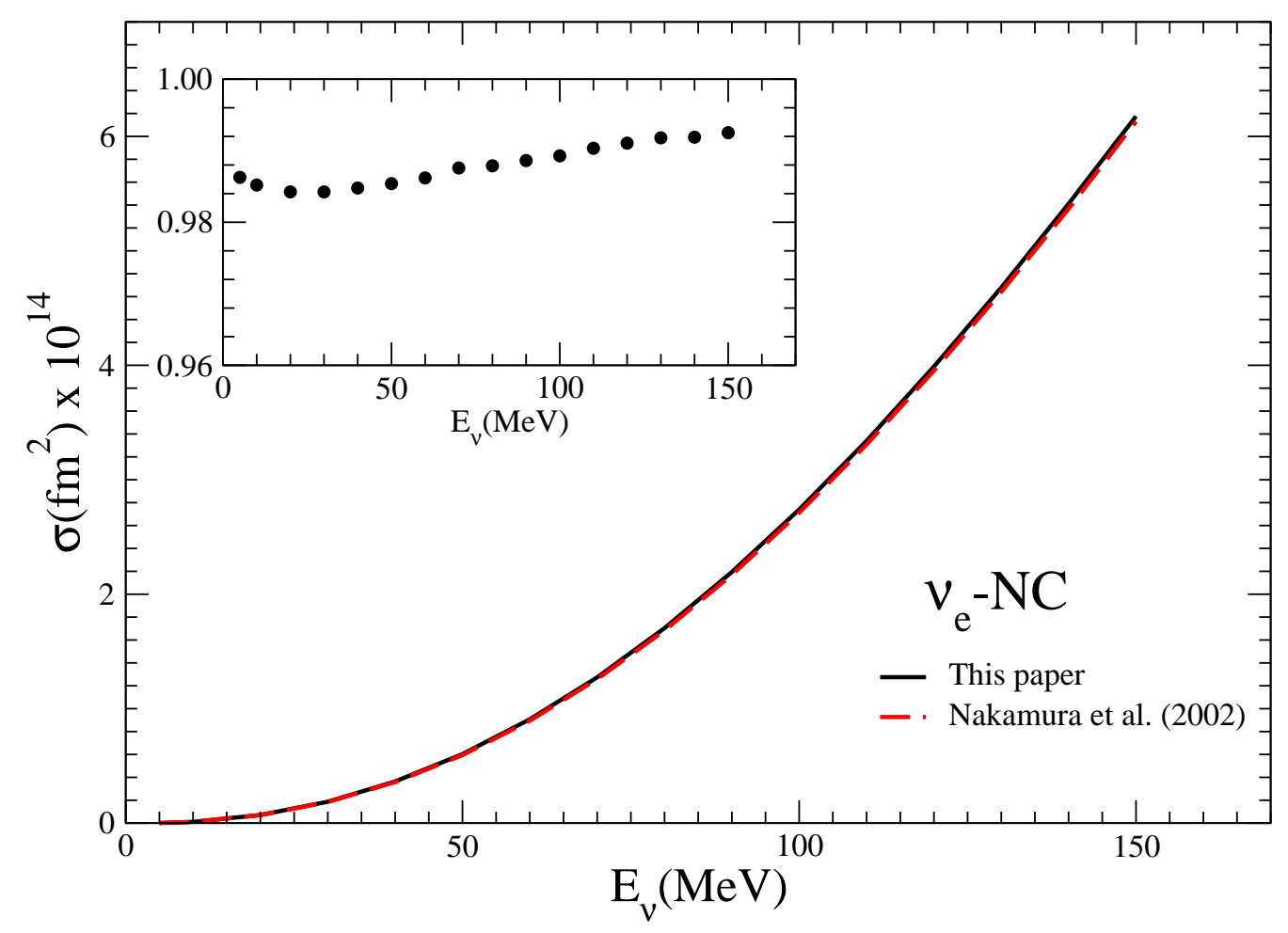

FIG. 29: Same as in Fig. 27 but for the $\nu_{e}$-NC induced process on the deuteron.

The cross sections increase rapidly, by over two orders of magnitude, as the neutrino energy increases from threshold to $150 \mathrm{MeV}$. Nevertheless, the present $\chi$ EFT predictions remain close to, albeit consistently larger at the $1-2 \%$ level than, those obtained in the conventional frameworks of Refs. [72] and [74], as shown explicitly for the case of Ref. [72] by the insets in Figs. 27-30 . The present $\chi$ EFT electro-weak current and the mesonexchange models adopted in Refs. [72] and [74] provide an excellent description of lowenergy observables in the two- and three-nucleon systems (see Refs. [18, 24] and references therein). In particular, the axial current in both approaches ( $\chi$ EFT and meson-exchange) is constrained to reproduce the tritium Gamow-Teller matrix element. 
TABLE 5: Total cross sections in $\mathrm{fm}^{2}$, corresponding to cutoff $\Lambda=500 \mathrm{MeV}$, for the CCinduced processes on the deuteron at selected initial neutrino energies and at increasing orders in the chiral counting. Referring to Figs. 23-26, the rows are labeled as follows: LO for the leading-order vector and axial current and charge; $\mathrm{N}(1 \mid 2) \mathrm{LO}$ including the vector current and axial charge at N1LO, and the axial current and vector charge at N2LO; N(2|3)LO including the vector current at N2LO, and the axial current and vector charge at N3LO; $\mathrm{N}(3 \mid 4) \mathrm{LO}$ including the vector current and axial charge at N3LO, and the axial current and vector charge at $\mathrm{N} 4 \mathrm{LO}$. Also listed are the results at $\mathrm{LO}$ and $\mathrm{N}(3 \mid 4) \mathrm{LO}$ but $\Lambda=600 \mathrm{MeV}$ (labeled as $\mathrm{LO}^{\star}$ and $\mathrm{N}(3 \mid 4) \mathrm{LO}^{\star}$ ), and those obtained in the conventional frameworks of (i) Ref. [74] in impulse approximation (IA) and with inclusion of two-body currents (TOT) and (ii) Ref. [72] with inclusion of two-body currents (TOT). The notation $(x x)$ means $10^{x x}$.

\begin{tabular}{c||c|c|c|c||c|c|c|c||}
\multicolumn{1}{c||}{} & \multicolumn{9}{c||}{$\sigma\left(\nu_{e}-\mathrm{CC}\right)$} \\
\hline \hline$E_{\nu}(\mathrm{MeV})$ & 10 & 50 & 100 & 150 & 10 & 50 & 100 & 150 \\
\hline $\mathrm{LO}$ & $2.676(-16)$ & $1.345(-14)$ & $6.611(-14)$ & $1.591(-13)$ & $1.243(-16)$ & $7.441(-15)$ & $2.661(-14)$ & $4.944(-14)$ \\
$\mathrm{N}(1 \mid 2) \mathrm{LO}$ & $2.670(-16)$ & $1.345(-14)$ & $6.606(-14)$ & $1.581(-13)$ & $1.237(-16)$ & $7.341(-15)$ & $2.602(-14)$ & $4.792(-14)$ \\
$\mathrm{N}(2 \mid 3) \mathrm{LO}$ & $2.794(-16)$ & $1.413(-14)$ & $6.913(-14)$ & $1.653(-13)$ & $1.298(-16)$ & $7.825(-15)$ & $2.801(-14)$ & $5.221(-14)$ \\
$\mathrm{N}(3 \mid 4) \mathrm{LO}$ & $2.734(-16)$ & $1.388(-14)$ & $6.852(-14)$ & $1.650(-13)$ & $1.266(-16)$ & $7.523(-15)$ & $2.676(-14)$ & $4.981(-14)$ \\
\hline $\mathrm{LO}^{\star}$ & $2.666(-16)$ & $1.342(-14)$ & $6.593(-14)$ & $1.588(-13)$ & $1.239(-16)$ & $7.417(-15)$ & $2.653(-14)$ & $4.925(-14)$ \\
$\mathrm{N}(3 \mid 4) \mathrm{LO} \mathrm{L}^{\star}$ & $2.729(-16)$ & $1.388(-14)$ & $6.858(-14)$ & $1.656(-13)$ & $1.263(-16)$ & $7.520(-15)$ & $2.679(-14)$ & $4.998(-14)$ \\
\hline IA Ref. [74] & $2.630(-16)$ & $1.314(-14)$ & $6.424(-14)$ & $1.516(-13)$ & $1.219(-16)$ & $7.260(-15)$ & $2.567(-14)$ & $4.688(-14)$ \\
TOT Ref. [74] & $2.680(-16)$ & $1.348(-14)$ & $6.631(-14)$ & $1.574(-13)$ & $1.242(-16)$ & $7.403(-15)$ & $2.606(-14)$ & $4.751(-14)$ \\
\hline TOT Ref. [72] & $2.708(-16)$ & $1.376(-14)$ & $6.836(-14)$ & $1.641(-13)$ & $1.242(-16)$ & $7.372(-15)$ & $2.618(-14)$ & $4.871(-14)$ \\
\hline \hline
\end{tabular}

The $\chi$ EFT cross sections of Figs. 27-30 correspond to cutoff $\Lambda=500$, but their variation as $\Lambda$ is increases to $600 \mathrm{MeV}$ remains well below $1 \%$ over the whole energy range, as can be seen in Tables 5 and 6 , rows labeled $\mathrm{N}(3 \mid 4) \mathrm{LO}$ and $\mathrm{N}(3 \mid 4) \mathrm{LO}^{\star}$. The convergence of the chiral expansion is also shown in these tables, where the various rows are labeled in accordance with the power counting adopted in the present chapter, see Figs. 27-30. Overall, corrections beyond LO lead to a couple of \% increase in the cross sections for both the CC and NC processes. A similar increase due to two-body terms in the weak current is obtained in the conventional calculations, see rows labeled IA and TOT in Tables 5 and 6 . Note that the IA row corresponds to results obtained with one-body currents, including relativistic corrections [74]. These IA currents are the same as the $\chi$ EFT ones illustrated by panel (a) of Fig. 23, panels (a) and (b) of Fig. 24, panels (a)-(d) of Fig. 25, and panel (a) of Fig. 26. Since the contributions due to the OPE two-body terms in the vector current, panels (b) and (c) of Fig. 23, and axial charge, panels (b) and (c) of Fig. 26, are very small, the difference between the IA and $\mathrm{N}(1 \mid 2) \mathrm{LO}$ results essentially reflects differences in the wave functions obtained from conventional and chiral potentials. Indeed, the overall $\sim 2 \%$ offset between 
the TOT and $\mathrm{N}(3 \mid 4) \mathrm{LO}$ predictions is primarily due to these differences.

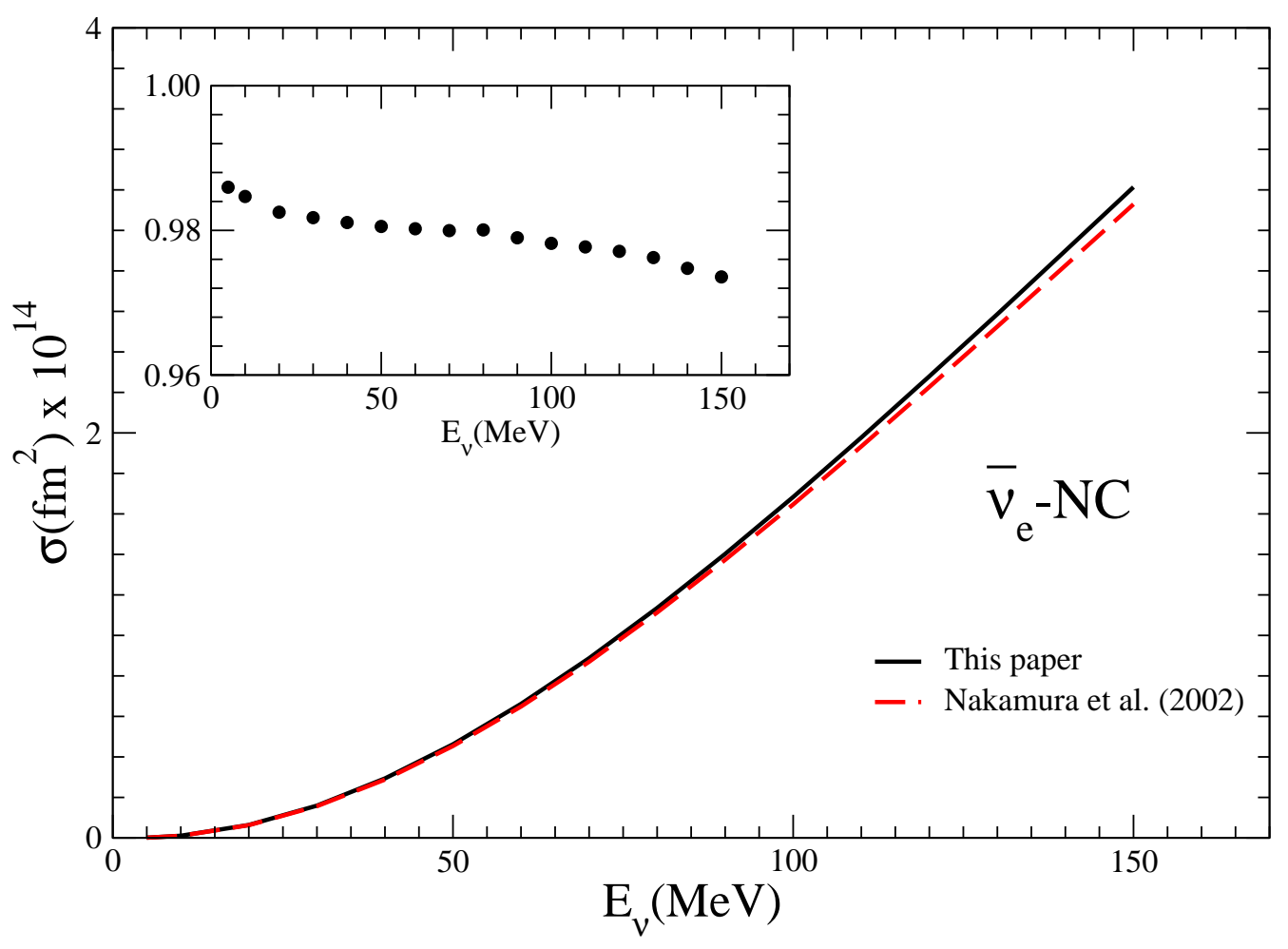

FIG. 30: Same as in Fig. 27 but for the $\bar{\nu}_{e}-\mathrm{NC}$ induced process on the deuteron.

TABLE 6: Same as in Table 5 but for the NC-induced processes.

\begin{tabular}{c||c|c|c|c||c|c|c|c||}
\multicolumn{1}{c||}{} & \multicolumn{9}{c||}{$\sigma\left(\nu_{e}-\mathrm{NC}\right)$} \\
\hline \hline$E_{\nu}(\mathrm{MeV})$ & 10 & 50 & 100 & 150 & 10 & 50 & 100 & 150 \\
\hline $\mathrm{LO}$ & $1.101(-16)$ & $5.872(-15)$ & $2.660(-14)$ & $5.991(-14)$ & $1.050(-16)$ & $4.554(-15)$ & $1.664(-14)$ & $3.175(-14)$ \\
$\mathrm{N}(1 \mid 2) \mathrm{LO}$ & $1.097(-16)$ & $5.856(-15)$ & $2.644(-14)$ & $5.912(-14)$ & $1.045(-16)$ & $4.505(-15)$ & $1.631(-14)$ & $3.076(-14)$ \\
$\mathrm{N}(2 \mid 3) \mathrm{LO}$ & $1.151(-16)$ & $6.178(-15)$ & $2.789(-14)$ & $6.250(-14)$ & $1.097(-16)$ & $4.793(-15)$ & $1.752(-14)$ & $3.347(-14)$ \\
$\mathrm{N}(3 \mid 4) \mathrm{LO}$ & $1.124(-16)$ & $6.032(-15)$ & $2.740(-14)$ & $6.176(-14)$ & $1.069(-16)$ & $4.625(-15)$ & $1.684(-14)$ & $3.214(-14)$ \\
\hline $\mathrm{LO}^{\star}$ & $1.096(-16)$ & $5.853(-15)$ & $2.652(-14)$ & $5.973(-14)$ & $1.045(-16)$ & $4.539(-15)$ & $1.659(-14)$ & $3.165(-14)$ \\
$\mathrm{N}(3 \mid 4) \mathrm{L} \mathrm{O}^{\star}$ & $1.121(-16)$ & $6.028(-15)$ & $2.742(-14)$ & $6.191(-14)$ & $1.067(-16)$ & $4.622(-15)$ & $1.685(-14)$ & $3.224(-14)$ \\
\hline IA Ref. [74] & $1.084(-16)$ & $5.747(-14)$ & $2.577(-14)$ & $5.720(-13)$ & $1.033(-16)$ & $4.449(-15)$ & $1.604(-14)$ & $3.003(-14)$ \\
TOT Ref. [74] & $1.104(-16)$ & $5.892(-15)$ & $2.657(-14)$ & $5.935(-14)$ & $1.053(-16)$ & $4.546(-15)$ & $1.640(-14)$ & $3.075(-14)$ \\
\hline TOT Ref. [72] & $1.107(-16)$ & $5.944(-14)$ & $2.711(-14)$ & $6.130(-13)$ & $1.053(-16)$ & $4.535(-15)$ & $1.647(-14)$ & $3.129(-14)$ \\
\hline \hline
\end{tabular}


The cross sections for the $\nu_{l}$-NC and $\bar{\nu}_{l}$-NC processes only differ in the sign of the interference response function $R_{x y}$ in Eq. (302). In the case $\nu_{e}$-CC and $\bar{\nu}_{e}$-CC processes, additional differences result from isospin-symmetry breaking terms in the final state interactions of $p p$ versus $n n$. At low energies $\left(E_{\nu} \lesssim 10 \mathrm{MeV}\right)$, cross sections are dominated by the axial current, the associated contributions being more than two orders of magnitude larger than those from the vector current. As the energy increases, vector-current contributions increase becoming comparable, albeit still significantly smaller by over a factor of five at $E_{\nu}=150$ $\mathrm{MeV}$ than, axial-current ones. As a consequence, the $\nu_{l}$ - $\mathrm{NC}$ and $\bar{\nu}_{l}$ - $\mathrm{NC}$ are fairly close at low energies, but diverge significantly from each other as the energy increases. Because of the aforementioned isospin-symmetry breaking effects (primarily induced by the Coulomb repulsion), the $\nu_{e}$-CC and $\bar{\nu}_{e}$-CC differ even at low energies. Finally, cross section contributions from the axial charge are negligible at $E_{\nu}=10 \mathrm{MeV}$ and remain well below $1 \%$ at $E_{\nu}=150 \mathrm{MeV}$. At this latter energy, for example, ignoring these axial-charge contributions altogether would reduce the $\nu_{l}$ - NC $\left(\bar{\nu}_{l}\right.$-NC) cross section from the $\mathrm{N}(3 \mid 4)$ LO value of 6.176 (3.214) listed in Table 6 to 6.157 (3.194) in units of $10^{-14} \mathrm{fm}^{2}$. Thus, uncertainties in the values of the LECs $c_{5,2}$ and $c_{5,3}$ in the contact axial charge do not have a significant impact on the present cross section predictions. 


\section{CHAPTER 8}

\section{CONCLUSIONS}

In the first part of this Thesis, after reviewing some key points of chiral effective field theory ( $\chi \mathrm{EFT})$, we have discussed the formalism used to derive nuclear potentials and currents form chiral Lagrangians. This formalism consists in essence of the following two steps: (i) time ordered perturbation theory is used to calculate the field-theory amplitude, and power counting is used to organize the various terms contributing to the amplitudes; (ii) nuclear potential and currents are obtained by requiring that when they are iterated into the corresponding Lippmann-Schwinger (or Schrödinger) equation they generate $T$-matrices that match the field-theory amplitudes on-the-energy-shell order-by-order in the power counting. As we have seen this prescription leads to the partial cancellations between the contributions of irreducible diagrams and those owing to nonstatic corrections from energy denominators of reducible diagrams.

In the second part of this Thesis, we have discussed in detail the derivation of nuclear potentials and currents up to one loop. In both derivations ultraviolet divergences associated with loop corrections are isolated in dimensional regularization and reabsorbed through a redefinition of LECs. The resulting axial current is finite and conserved in the chiral limit, while the axial charge requires renormalization. For the axial current there is a single LEC $z_{0}$ which enters at N3LO, while for the axial charge four independent LECs have been found to be present at N4LO. Loop corrections to the one-pion exchange (OPE) axial charge lead to renormalization of linear combinations of the LECs $d_{i}$ in the subleading $\mathcal{L}_{\pi N}^{(3)}$ Lagrangian.

In the third part of this Thesis, we have reported two applications. The first is the calculation of the tritium $\beta$-decay rate by including in the charge-changing weak current the corrections up to one loop (N4LO) derived in Ch. 5. The LEC $z_{0}$ in the axial current has been constrained by reproducing the empirical value for tritium Gamow-Teller (GT) matrix element. Using trinucleon wave functions obtained from solutions of the Schrödinger equation with two- and three-nucleon potentials corresponding to either $\chi \mathrm{EFT}$ (the N3LO/N2LO combination $[63,83]$ ) or meson-exchange phenomenology (the AV18/UIX combination) we have found that the contributions due to loop corrections in the axial current are, in relative terms, as large as those from the one-pion exchange, which nominally occur at lower order in the power counting. 
The second application has been the calculation of the inclusive cross sections for the reactions ${ }^{2} \mathrm{H}\left(\nu_{e}, e^{-}\right) p p$ and ${ }^{2} \mathrm{H}\left(\nu_{e}, e^{+}\right) n n$, and ${ }^{2} \mathrm{H}\left(\nu_{l} / \overline{\nu_{l}}, \nu_{l}^{\prime} / \overline{\nu_{l}}\right) n p$. The results are within 1-2\%, albeit consistently larger than, those obtained in earlier conventional calculations. The weak dependence on the cutoff suggests that the associated theoretical uncertainty is very small.

The operators derived in this Thesis can be used to improve the accuracy and reliability of theoretical predictions for weak nuclear reactions, for example the proton weak captures on protons and ${ }^{3} \mathrm{He}$ important in solar physics. Earlier calculations of these processes, such as those reported in Refs. [48, 84], have used axial current operators up to N3LO. In view of the relatively large contributions obtained here at N4LO for the tritium Gamow-Teller matrix element, it would be interesting to reexamine these capture reactions by including these N4LO loop corrections.

Finally, we note that an accurate theory of nuclear electroweak structure and dynamics is relevant for low-energy tests of physics beyond the standard model in $\beta$-decay experiments [85]. Phenomenologically, the weak interactions are known to couple only to lefthanded neutrinos and to violate parity maximally. However beyond-the-standard-model (BSM) theories have been constructed in which small deviations from these properties are introduced. These deviations affect the correlation coefficients entering the $\beta$-decay rates and can, in principle, be detected. For a proper interpretation of these measurements and, in particular, to unravel possible signatures of BSM physics, it is crucial to have control of the nuclear structure and weak interactions in nuclei.

When coupled to numerically exact methods such as quantum Monte Carlo techniques, to solve the many-body Schrödinger equations, the $\chi \mathrm{EFT}$ potentials and weak currents obtained in this Thesis offer the opportunity to carry out a first-principles calculation of these decays and provide predictions for their rates and associated theoretical uncertainties rooted in QCD. 


\section{REFERENCES}

[1] J. Carlson, S. Gandolfi, F. Pederiva, Steven C. Pieper, R. Schiavilla, K. E. Schmidt, ad R. B. Wiringa Rev. Mod. Phys. 87, 1067 (2015).

[2] K. G. Wilson, Phys. Rev. D 10, 2445 (1974).

[3] S. R. Beane, W. Detmold, K. Orginos, and M. J. Savage Prog. Part. Nucl. Phys. 66, 1-40 (2011).

[4] S. Weinberg, Physica A 96, 327 (1979).

[5] S. Weinberg, Phys. Lett. B 251, 288 (1990); Nucl. Phys. B 363, 3 (1991); Phys. Lett. B 295, 114 (1992).

[6] C. Ordónez, and U. van Kolck, Phys. Lett. B 291, 459 (1992).

[7] N. Kaiser, R. Brockmann, and W. Weise Nucl. Phys. A 625, 758 (1977).

[8] E. Epelbaum, W. Gloeckle, and U.-G. Meissner, Nucl. Phys. A 637, 107 (1998).

[9] D. R. Entem, and R. Machleidt, Phys. Rev. C 68, 041001(R) (2003).

[10] E. Epelbaum, H. Krebs, and Ulf-G. Meissner, Phys. Rev. Lett. 115, 122301 (2015).

[11] M. Piarulli, L. Girlanda, R. Schiavilla, R. Navarro Pérez, J. E. Amaro, and E. Ruiz Arriola Phys. Rev. C 91, 024003 (2015).

[12] E. Epelbaum, A. Nogga, W.Glöckle,H. Kamada, Ulf-G. Meissner, and H. Witala Phys. Rev. C 66, 064001 (2002).

[13] E. Epelbaum, Phys. Lett. B, 639, 456-461 (2006).

[14] V. Bernard, E. Epelbaum, H. Krebs, and Ulf-G. Meissner, Phys. Rev. C 84, 054001 (2011).

[15] H. Krebs, A. Gasparyan, and E. Epelbaum, Phys. Rev. C 87, 054007 (2013).

[16] Skibiński, J. Golak, K. Topolnicki, H. Witala, E. Epelbaum, W. Glöckle, H. Krebs, A. Nogga, and H. Kamada, Phys. Rev. C 84, 054005 (2011).

[17] E. Epelbaum, H. W. Hammer, and U-G. Meissner, Rev. Mod. Phys. 81, 1773 (2009). 
[18] L.E. Marcucci, F. Gross, M.T. Pena, M. Piarulli, R. Schiavilla, I. Sick, A. Stadler, J.W. Van Orden, and M. Viviani, J. Phys. G: Nucl. Part. Phys. 43, 023002 (2016).

[19] S. Bacca and S. Pastore, J. Phys. G 41, no. 12, 123002 (2014).

[20] T.-S. Park, D.-P. Min, and M. Rho, Nucl. Phys. A 596, 515 (1996).

[21] S. Pastore, R. Schiavilla, and J.L. Goity, Phys. Rev. C 78, 064002 (2008).

[22] S. Pastore, L. Girlanda, R. Schiavilla, M. Viviani, and R.B. Wiringa, Phys. Rev. C 80, 034004 (2009).

[23] S. Pastore, L. Girlanda, R. Schiavilla, and M. Viviani, Phys. Rev. C 84, 024001 (2011).

[24] M. Piarulli, L. Girlanda, L.E. Marcucci, S. Pastore, R. Schiavilla, and M. Viviani, Phys. Rev. C 87, 014006 (2013).

[25] S. Kolling, E. Epelbaum, H. Krebs, and U.-G. Meissner Phys. Rev. C 80, 045502 (2009).

[26] S. Kolling, E. Epelbaum, H. Krebs, and U.-G. Meissner Phys. Rev. C 84, 054008 (2011).

[27] S. Kölling, E. Epelbaum, and D. R. Phillips, Phys. Rev. C 86, 047001 (2011).

[28] T.-S. Park, D.-P. Min, and M. Rho, Phys. Rep. 233, 341 (1993).

[29] Q.R. Ahmad et al. (SNO Collaboration), Phys. Rev. Lett. 89, 011301 (2002); S.N. Ahmed et al. (SNO Collaboration), Phys. Rev. Lett. 92, 181301 (2004).

[30] J.N. Bahcall and M.H. Pinsonneault, Phys. Rev. Lett. 92, 121301 (2004).

[31] S. Weinberg, Phys. Rev. Lett. 17, 616 (1966).

[32] F. Gross, Relativistic Quantum Mechanics and Field Theory (Wiley, New York, 1993).

[33] C. Amsler et al. (Particle Data Group), Phys. Lett. B 667, 1 (2008).

[34] S. Scherer, and M. R. Schindler, A Primer for Chiral Perturbation Theory (SpringerVerlag, Heidelberg, 2012).

[35] J. Gasser and H. Leutwyler, Ann. Phys. (N.Y.) 158, 142 (1984).

[36] J. Gasser and H. Leutwyler, Nucl. Phys. B 250, 465 (1985).

[37] H. Leutwyler, Ann. Phys. 235, 165 (1994). 
[38] S. R. Coleman, J. Wess, and B. Zumino, Phys. Rev. 177, 2239 (1969).

[39] C. G. Callan, S. R. Coleman, J. Wess, and B. Zumino, Phys. Rev. 177, 2247 (1969).

[40] R. Haag, Phys. Rev. 112, 669 (1958).

[41] N. Fettes, U.-G. Meissner, M. Mojzis, and S. Steininger, Ann. Phys. (N.Y.) 283, 273 (2000).

[42] L. Girlanda, S. Pastore, R. Schiavilla, and M. Viviani, Phys. Rev. C 81, 034005 (2010).

[43] J.L. Friar, Ann. Phys. (N.Y.) 104, 380 (1977).

[44] E. Epelbaum, W. Glöckle, and U.-G. Meissner, Nucl. Phys. A 671, 295 (2000); Nucl. Phys. A 714,535 (2003); Nucl. Phys. A 747, 362 (2005).

[45] S. Okubo, Prog. Theor. Phys. 12, 603 (1954).

[46] C. Ordonez, L. Ray, and U. van Kolck Phys. Rev. C 53, 5 (1996).

[47] M. Viviani, A. Baroni, L. Girlanda, A. Kievsky, L.E. Marcucci, and R. Schiavilla, Phys. Rev. C 89, 064004 (2014).

[48] T.-S. Park, L.E. Marcucci, R. Schiavilla, M. Viviani, A. Kievsky, S. Rosati, K. Kubodera, D.-P. Min, and M. Rho, Phys. Rev. C 67, 055206 (2003).

[49] H. Krebs, E. Epelbaum, and U.-G. Meissner, arXiv:1610.03569.

[50] M.R. Schindler, T. Fuchs, J. Gegelia, and S. Scherer, Phys. Rev. C 75, 025202 (2007).

[51] K. Kubodera, J. Delorme, and Mannque Rho, Phys. Rev. Lett. 40, 755 (1978).

[52] A. Gardestig and D.R. Phillips, Phys. Rev. Lett. 96, 232301 (2006).

[53] J. Gasser, M.A. Ivanov, E. Lipartia, M. Mojzis, and A. Rusetsky, Eur. Phys. J. C 26,13 (2002).

[54] D. Gazit, S. Quaglioni, and P. Navratil, Phys. Rev. Lett. 103, 102502 (2009).

[55] L.E. Marcucci, A. Kievsky, S. Rosati, R. Schiavilla, and M. Viviani, Phys. Rev. Lett. 108, 052502 (2012). 
[56] R. Schiavilla, V. G. J. Stoks, W. Glöckle, H. Kamada, A. Nogga, J. Carlson, R. Machleidt, V. R. Pandharipande, R. B. Wiringa, A. Kievsky, S. Rosati, and M. Viviani, Phys. Rev. C 58, 1263 (1998).

[57] K.A. Olive et al. (Particle Data Group), Chin. Phys. C 38, 090001 (2014).

[58] S. Raman, C.A. Houser, T. A. Walkiewicz, and I.S. Towner, At. Data and Nucl. Data Tables 21, 567 (1978).

[59] J. J. Simpson, Phys. Rev. C 35, 752 (1987).

[60] J. C. Hardy, and I. S. Towner, Phys. Rev. C 91, 025501 (2015).

[61] A. Kievsky, S. Rosati, M. Viviani, L. E. Marcucci, and L. Girlanda, J. Phys. G: Nucl. Part. Phys. 35, 063101 (2008).

[62] R. Machleidt and D.R. Entem, Phys. Rep. 503, 1 (2011).

[63] P. Navratil, Few-Body Syst. 41, 117 (2007).

[64] R. B. Wiringa, V. G. J. Stoks, and R. Schiavilla, Phys. Rev. C 51, 38 (1995).

[65] B. S. Pudliner, V. R. Pandharipande, J. Carlson, and R. B. Wiringa, Phys. Rev. Lett. 74, 4396 (1995).

[66] M. Hoferichter, J. Ruiz de Elvira, B. Kubis, and U.-G. Meissner, Phys. Rev. Lett. 115, 192301 (2015).

[67] R. Schiavilla, V. R. Pandharipande, and D. O. Riska, Phys. Rev. C 40, 2294 (1989).

[68] L. E. Marcucci, M. Piarulli, M. Viviani, L. Girlanda, A. Kievsky, S. Rosati, and R. Schiavilla, Phys. Rev. C 83, 014002 (2011).

[69] K. Schoen, D. L. Jacobson, M. Arif, P. R. Huffman, T. C. Black, W. M. Snow, S. K. Lamoreaux, H. Kaiser, and S. A. Werner, Phys. Rev. C 67, 044005 (2003).

[70] A. Nogga, A. Kievsky, H. Kamada, W. Glöckle, L. E. Marcucci, S. Rosati, and M. Viviani, Phys. Rev. C 67, 034004 (2003).

[71] S. Nakamura, T. Sato, V. Gudkov, and K. Kubodera, Phys. Rev. C 63, 034617 (2001). 
[72] S. Nakamura et al., Nucl. Phys. A707, 561 (2002); http://www-nuclth.phys.sci.osakau.ac.jp/top/Netal/total/index.html .

[73] M. Butler, J.-W. Chen, and X. Kong, Phys. Rev. C 63, 035501 (2001).

[74] G. Shen, L.E. Marcucci, J. Carlson, S. Gandolfi, and R. Schiavilla, Phys. Rev. C 86, 035503 (2012).

[75] A. Baroni, L. Girlanda, S. Pastore, R. Schiavilla, and M. Viviani, Phys. Rev. C 93, 015501 (2016); ibidem, 049902(E) (2016).

[76] A. Baroni, L. Girlanda, A. Kievsky, L.E. Marcucci, R. Schiavilla, and M. Viviani, Phys. Rev. C 94, 024003 (2016).

[77] K. Nakamura et al. (Particle Data Group), J. Phys. G 37, 075021 (2010).

[78] I.S. Towner, Phys. Rev. C 58, 1288 (1998).

[79] A. Kurylov, M.J. Ramsey-Musolf, and P. Vogel, Phys. Rev. C 65, 055501 (2002); Phys. Rev. C 67, 035502 (2003).

[80] D.R. Phillips Phys. Lett. B 567, 12 (2003).

[81] C.E. Carlson, Phys. Rev. D 34, 2704 (1986).

[82] B. Kubis and U.-G. Meissner, Nucl. Phys. A679, 698 (2001)

[83] D. R. Entem, and R. Machleidt, Phys. Lett. B 524, 93. (2002).

[84] L.E. Marcucci, R. Schiavilla, and M. Viviani, Phys. Rev. Lett. 110, 192503 (2013).

[85] N. Severijns, M. Beck, and O. Naviliat-Cuncic, Rev. Mod. Phys. 78, 991 (2006).

[86] I.S. Gerstein, R. Jackiw, B.W. Lee, and S. Weinberg, Phys. Rev. D 3, 2486 (1971). 


\section{APPENDIX A}

\section{CHIRAL LAGRANGIANS}

We summarize the notation and conventions for the various fields and covariant derivatives adopted in this Thesis [41]:

$$
\begin{aligned}
U & =1+\frac{i}{f_{\pi}} \boldsymbol{\tau} \cdot \boldsymbol{\pi}-\frac{1}{2 f_{\pi}^{2}} \boldsymbol{\pi}^{2}-\frac{i \alpha}{f_{\pi}^{3}} \boldsymbol{\pi}^{2} \boldsymbol{\tau} \cdot \boldsymbol{\pi}+\frac{8 \alpha-1}{8 f_{\pi}^{4}} \boldsymbol{\pi}^{4}+\ldots, \\
u & =\sqrt{U} \\
& =1+\frac{i}{2 f_{\pi}} \boldsymbol{\tau} \cdot \boldsymbol{\pi}-\frac{1}{8 f_{\pi}^{2}} \boldsymbol{\pi}^{2}-\frac{i(8 \alpha-1)}{16 f_{\pi}^{3}} \boldsymbol{\pi}^{2} \boldsymbol{\tau} \cdot \boldsymbol{\pi}+\frac{(32 \alpha-5)}{128 f_{\pi}^{4}} \boldsymbol{\pi}^{4}+\ldots, \\
u_{\mu} & =i\left[u^{\dagger}\left(\partial_{\mu}-i r_{\mu}\right) u-u\left(\partial_{\mu}-i l_{\mu}\right) u^{\dagger}\right], \\
D_{\mu} U & =\partial_{\mu} U-i r_{\mu} U+i U l_{\mu}, \\
D_{\mu} N & =\left(\partial_{\mu}+\Gamma_{\mu}\right) N=\partial_{\mu} N+\frac{1}{2}\left[u^{\dagger}\left(\partial_{\mu}-i r_{\mu}\right) u+u\left(\partial_{\mu}-i l_{\mu}\right) u^{\dagger}\right] N, \\
F_{\mu \nu}^{ \pm} & =u^{\dagger} F_{\mu \nu}^{R} u \pm u F_{\mu \nu}^{L} u^{\dagger}, \\
F_{\mu \nu}^{R} & =\partial_{\mu} r_{\nu}-\partial_{\nu} r_{\mu}-i\left[r_{\mu}, r_{\nu}\right], \quad r_{\mu}=v_{\mu}+a_{\mu}, \\
F_{\mu \nu}^{L} & =\partial_{\mu} l_{\nu}-\partial_{\nu} l_{\mu}-i\left[l_{\mu}, l_{\nu}\right], \quad l_{\mu}=v_{\mu}-a_{\mu}, \\
\chi_{ \pm} & =u^{\dagger} \chi u \pm u \chi^{\dagger} u=m_{\pi}^{2}\left(U^{\dagger} \pm U\right) .
\end{aligned}
$$

The parameter $\alpha$ is arbitrary because of the freedom in the choice of pion field - the only constraint is that $U$ be unitary with $\operatorname{det} U=1$. In the following we consider only the coupling to the axial-vector field; further, we ignore isospin-symmetry-breaking effects as well as the coupling to the isoscalar component of the axial-vector field, and hence

$$
\begin{aligned}
r_{\mu} & =-l_{\mu}=\frac{1}{2} \boldsymbol{\tau} \cdot \mathbf{A}_{\mu} \\
F_{\mu \nu}^{R} & =\frac{1}{2} \boldsymbol{\tau} \cdot\left(\partial_{\mu} \mathbf{A}_{\nu}-\partial_{\nu} \mathbf{A}_{\mu}+\mathbf{A}_{\mu} \times \mathbf{A}_{\nu}\right) \\
F_{\mu \nu}^{L} & =-\frac{1}{2} \boldsymbol{\tau} \cdot\left(\partial_{\mu} \mathbf{A}_{\nu}-\partial_{\nu} \mathbf{A}_{\mu}-\mathbf{A}_{\mu} \times \mathbf{A}_{\nu}\right) .
\end{aligned}
$$


Inserting the expansions for $U$ and $u$ and keeping terms linear in the axial-vector field, we find:

$$
\begin{aligned}
u_{\mu}= & -\frac{1}{f_{\pi}}\left(1-\frac{\alpha}{f_{\pi}^{2}} \boldsymbol{\pi}^{2}\right) \boldsymbol{\tau} \cdot \partial_{\mu} \boldsymbol{\pi}+\frac{4 \alpha-1}{2 f_{\pi}^{3}} \boldsymbol{\tau} \cdot \boldsymbol{\pi} \boldsymbol{\pi} \cdot \partial_{\mu} \boldsymbol{\pi} \\
& +\boldsymbol{\tau} \cdot \mathbf{A}_{\mu}+\frac{1}{2 f_{\pi}^{2}}[(\boldsymbol{\tau} \times \boldsymbol{\pi}) \times \boldsymbol{\pi}] \cdot \mathbf{A}_{\mu}+\ldots, \\
D_{\mu} U= & i \boldsymbol{\tau} \cdot\left[\frac{1}{f_{\pi}} \partial_{\mu} \boldsymbol{\pi}-\left(1-\frac{\boldsymbol{\pi}^{2}}{2 f_{\pi}^{2}}\right) \mathbf{A}_{\mu}\right]-\frac{1}{f_{\pi}^{2}} \boldsymbol{\pi} \cdot \partial_{\mu} \boldsymbol{\pi}+\frac{1}{f_{\pi}} \boldsymbol{\pi} \cdot \mathbf{A}_{\mu}+\ldots, \\
D_{\mu} N= & {\left[\partial_{\mu}+\frac{i}{4 f_{\pi}^{2}}(\boldsymbol{\tau} \times \boldsymbol{\pi}) \cdot \partial_{\mu} \boldsymbol{\pi}-\frac{i}{2 f_{\pi}}\left(1-\alpha \frac{\boldsymbol{\pi}^{2}}{f_{\pi}^{2}}\right)(\boldsymbol{\tau} \times \boldsymbol{\pi}) \cdot \mathbf{A}_{\mu}\right.} \\
& \left.+i \frac{(8 \alpha-1)}{16 f_{\pi}^{4}} \boldsymbol{\pi}^{2} \partial_{\mu} \boldsymbol{\pi} \cdot(\boldsymbol{\pi} \times \boldsymbol{\tau})+\ldots\right] \\
F_{\mu \nu}^{+}= & \frac{1}{f_{\pi}}(\boldsymbol{\tau} \times \boldsymbol{\pi}) \cdot \mathbf{F}_{\mu \nu}+\ldots, \\
F_{\mu \nu}^{-}= & {\left[\boldsymbol{\tau}+\frac{1}{2 f_{\pi}^{2}}(\boldsymbol{\tau} \times \boldsymbol{\pi}) \times \boldsymbol{\pi}\right] \cdot \mathbf{F}_{\mu \nu}+\ldots, } \\
\chi_{+}= & m_{\pi}^{2}\left(2-\frac{\boldsymbol{\pi}^{2}}{f_{\pi}^{2}}\right)+\ldots, \\
\chi_{-}= & -\frac{2 i}{f_{\pi}} m_{\pi}^{2} \boldsymbol{\tau} \cdot \boldsymbol{\pi}+\ldots,
\end{aligned}
$$

where $\mathbf{F}_{\mu \nu} \equiv \partial_{\mu} \mathbf{A}_{\nu}-\partial_{\nu} \mathbf{A}_{\mu}$ and the ... denote higher powers of the pion field than shown.

\section{A.1 $\pi \pi$ SECTOR}

The $\pi \pi$ Lagrangians up to order $Q^{4}$ read [34]:

$$
\begin{aligned}
\mathcal{L}_{\pi \pi}^{(2)}= & \frac{f_{\pi}^{2}}{4}\left\langle D_{\mu} U\left(D^{\mu} U\right)^{\dagger}+\chi_{+}\right\rangle \\
\mathcal{L}_{\pi \pi}^{(4)}= & \frac{l_{1}}{4}\left\langle D_{\mu} U\left(D^{\mu} U\right)^{\dagger}\right\rangle\left\langle D_{\nu} U\left(D^{\nu} U\right)^{\dagger}\right\rangle+\frac{l_{2}}{4}\left\langle D_{\mu} U\left(D_{\nu} U\right)^{\dagger}\right\rangle\left\langle D^{\mu} U\left(D^{\nu} U\right)^{\dagger}\right\rangle+\frac{l_{3}}{16}\left\langle\chi_{+}\right\rangle^{2} \\
& +\frac{l_{4}}{16}\left[2\left\langle D_{\mu} U\left(D^{\mu} U\right)^{\dagger}\right\rangle\left\langle\chi_{+}\right\rangle+2\left\langle\chi^{\dagger} U \chi^{\dagger} U+\chi U^{\dagger} \chi U^{\dagger}\right\rangle-\left\langle\chi_{-}\right\rangle^{2}-4\left\langle\chi^{\dagger} \chi\right\rangle\right] \\
& +l_{5}\left(\left\langle F_{\mu \nu}^{R} U F_{L}^{\mu \nu} U^{\dagger}\right\rangle-\frac{1}{2}\left\langle F_{\mu \nu}^{L} F_{L}^{\mu \nu}+F_{\mu \nu}^{R} F_{R}^{\mu \nu}\right\rangle\right) \\
& +i \frac{l_{6}}{2}\left\langle F_{\mu \nu}^{R} D^{\mu} U\left(D^{\nu} U\right)^{\dagger}+F_{\mu \nu}^{L}\left(D^{\mu} U\right)^{\dagger} D^{\nu} U\right\rangle-\frac{l_{7}}{16}\left\langle\chi_{-}\right\rangle^{2}+\frac{h_{1}+h_{3}}{4}\left\langle\chi \chi^{\dagger}\right\rangle \\
& +\frac{h_{1}-h_{3}}{16}\left(\left\langle\chi_{+}\right\rangle^{2}+\left\langle\chi_{-}\right\rangle^{2}-2\left\langle\chi U^{\dagger} \chi U^{\dagger}+U \chi^{\dagger} U \chi^{\dagger}\right\rangle\right) \\
& -2 h_{2}\left\langle F_{\mu \nu}^{L} F_{L}^{\mu \nu}+F_{\mu \nu}^{R} F_{R}^{\mu \nu}\right\rangle
\end{aligned}
$$

where in the absence of isospin symmetry breaking (which is assumed throughout the present Thesis) $\chi$ is proportional to the identity matrix, namely $\chi=m_{\pi}^{2}$, and $\left\langle\chi_{-}\right\rangle$vanishes. Here 
$\langle\ldots\rangle$ implies a trace in flavor space. Furthermore, the terms proportional to the LECs $l_{1}, l_{2}$, $l_{5}, l_{6}$, and $h_{i}$ do not contribute to the order of interest. The symmetric matrices $\widetilde{G}_{a b}, G_{a b}$, $H_{a b}$, and $F_{a b}$ in the Lagrangian of Eq. (68) are obtained as

$$
\begin{aligned}
\widetilde{G}_{a b} & =\left(1-\frac{2 \alpha}{f_{\pi}^{2}} \pi^{2}+2 l_{4} \frac{m_{\pi}^{2}}{f_{\pi}^{2}}\right) \delta_{a b}-\frac{4 \alpha-1}{f_{\pi}^{2}} \pi_{a} \pi_{b}, \\
G_{a b} & =\widetilde{G}_{a b}+2 \frac{c_{2}+c_{3}}{f_{\pi}^{2}} \bar{N} N \delta_{a b}, \\
H_{a b} & =\left[1-\frac{8 \alpha-1}{4 f_{\pi}^{2}} \pi^{2}+2\left(l_{3}+l_{4}\right) \frac{m_{\pi}^{2}}{f_{\pi}^{2}}\right] \delta_{a b}, \\
F_{a b} & =\left(1-\frac{2 \alpha+1}{2 f_{\pi}^{2}} \pi^{2}+2 l_{4} \frac{m_{\pi}^{2}}{f_{\pi}^{2}}\right) \delta_{a b}-\frac{2 \alpha-1}{f_{\pi}^{2}} \pi_{a} \pi_{b} .
\end{aligned}
$$

By retaining only terms linear in the pion field and external axial field, the equation of motion implied by $\mathcal{L}_{\pi \pi}^{(2)}$ is

$$
\partial_{0}^{2} \boldsymbol{\pi}=-\left(\partial^{i} \partial_{i}+m_{\pi}^{2}\right) \boldsymbol{\pi}+f_{\pi} \partial_{0} \mathbf{A}^{0}+f_{\pi} \partial_{i} \mathbf{A}^{i}
$$

\section{A.2 $\pi N$ SECTOR}

The $\pi N$ Lagrangians up to order $Q^{3}$ read:

$$
\begin{aligned}
\mathcal{L}_{\pi N}^{(1)} & =\bar{N}\left(i \not D-m+\frac{g_{A}}{2} \psi \gamma_{5}\right) N \\
\mathcal{L}_{\pi N}^{(2)} & =\sum_{i=1}^{7} c_{i} \bar{N} O_{i}^{(2)} N, \\
\mathcal{L}_{\pi N}^{(3)} & =\sum_{i=1}^{23} d_{i} \bar{N} O_{i}^{(3)} N
\end{aligned}
$$

with the operators $O_{i}^{(2)}$ and $O_{i}^{(3)}$ defined as in Ref. [41]. Here $g_{A}$ is the nucleon axial coupling constant, and the $c_{i}$ and $d_{i}$ are LECs. Below, the $\gamma^{\mu}, \gamma_{5}$, and $\sigma^{\mu \nu}$ are $\gamma$ matrices and combinations of $\gamma$-matrices in standard notation [32], and $\epsilon^{\mu \nu \rho \sigma}$ is the Levi-Civita tensor with $\epsilon^{0123}=+1$. 
In terms of the expansions above, $\mathcal{L}_{\pi N}^{(1)}$ is given by

$$
\begin{aligned}
\mathcal{L}_{\pi N}^{(1)}= & \bar{N}\left[i \not \partial-m-\frac{1}{4 f_{\pi}^{2}}(\boldsymbol{\tau} \times \boldsymbol{\pi}) \cdot \not \partial \boldsymbol{\pi}-\frac{g_{A}}{2 f_{\pi}}\left(1-\frac{\alpha}{f_{\pi}^{2}} \boldsymbol{\pi}^{2}\right) \boldsymbol{\tau} \cdot \not \partial \boldsymbol{\pi} \gamma_{5}\right. \\
& +\frac{g_{A}}{4 f_{\pi}^{3}}(4 \alpha-1) \boldsymbol{\tau} \cdot \boldsymbol{\pi} \boldsymbol{\pi} \cdot \not \partial \boldsymbol{\pi} \gamma_{5}+\frac{(1-8 \alpha)}{16 f_{\pi}^{4}} \boldsymbol{\pi}^{2} \not \partial \boldsymbol{\pi} \cdot(\boldsymbol{\pi} \times \boldsymbol{\tau}) \\
& \left.+\frac{1}{2 f_{\pi}}\left(1-\frac{\alpha}{f_{\pi}^{2}} \boldsymbol{\pi}^{2}\right)(\boldsymbol{\tau} \times \boldsymbol{\pi}) \cdot \not \mathbf{A}+\frac{g_{A}}{2} \boldsymbol{\tau} \cdot \not{A} \gamma_{5}+\frac{g_{A}}{4 f_{\pi}^{2}}[(\boldsymbol{\tau} \times \boldsymbol{\pi}) \times \boldsymbol{\pi}] \cdot \mathbf{A} \gamma_{5}\right] N,
\end{aligned}
$$

where $\not \partial=\gamma^{\mu} \partial_{\mu}$ and $\mathbb{A}=\gamma^{\mu} \mathbf{A}_{\mu}$. The operators $O_{i}^{(2)}$ in the $\mathcal{L}_{\pi N}^{(2)}$ Lagrangian are expressed as (below the notation $\tilde{\chi}_{+}=\chi_{+}-\left\langle\chi_{+}\right\rangle / 2$ is used)

$$
\begin{aligned}
O_{1}^{(2)}= & \left\langle\chi_{+}\right\rangle \longrightarrow 4 m_{\pi}^{2}\left(1-\frac{\boldsymbol{\pi}^{2}}{2 f_{\pi}^{2}}\right), \\
O_{2}^{(2)}= & -\frac{1}{8 m^{2}}\left\langle u_{\mu} u_{\nu}\right\rangle D^{\mu \nu}+\text { h.c. } \longrightarrow \frac{1}{f_{\pi}^{2}} \partial_{0} \boldsymbol{\pi} \cdot \partial_{0} \boldsymbol{\pi}-\frac{2}{f_{\pi}} \partial_{0} \boldsymbol{\pi} \cdot \mathbf{A}_{0} \\
& \quad+\frac{1}{m f_{\pi}}\left(\frac{1}{f_{\pi}} \partial_{0} \boldsymbol{\pi} \cdot \partial_{i} \boldsymbol{\pi}-\partial_{0} \boldsymbol{\pi} \cdot \mathbf{A}_{i}-\partial_{i} \boldsymbol{\pi} \cdot \mathbf{A}_{0}\right) \gamma^{0} i \overleftrightarrow{\partial}^{i}, \\
O_{3}^{(2)}= & \frac{1}{2}\left\langle u_{\mu} u^{\mu}\right\rangle \longrightarrow \frac{1}{f_{\pi}^{2}} \partial_{\mu} \boldsymbol{\pi} \cdot \partial^{\mu} \boldsymbol{\pi}-\frac{2}{f_{\pi}} \partial_{\mu} \boldsymbol{\pi} \cdot \mathbf{A}^{\mu}, \\
O_{4}^{(2)}= & \frac{i}{4}\left[u_{\mu}, u_{\nu}\right] \sigma^{\mu \nu} \longrightarrow \frac{1}{2} \boldsymbol{\tau} \cdot\left(-\frac{1}{f_{\pi}^{2}} \partial_{\mu} \boldsymbol{\pi} \times \partial_{\nu} \boldsymbol{\pi}+\frac{2}{f_{\pi}} \mathbf{A}_{\mu} \times \partial_{\nu} \boldsymbol{\pi}\right) \sigma^{\mu \nu}, \\
O_{5}^{(2)}= & \widetilde{\chi}_{+} \longrightarrow 0, \\
O_{6}^{(2)}= & \frac{1}{8 m} F_{\mu \nu}^{+} \sigma^{\mu \nu} \longrightarrow \frac{1}{4 m f_{\pi}}(\boldsymbol{\tau} \times \boldsymbol{\pi}) \cdot \partial_{\mu} \mathbf{A}_{\nu} \sigma^{\mu \nu}, \\
O_{7}^{(2)}= & \frac{1}{8 m}\left\langle F_{\mu \nu}^{+}\right\rangle \sigma^{\mu \nu} \longrightarrow 0,
\end{aligned}
$$


while those in the $\mathcal{L}_{\pi N}^{(3)}$ Lagrangian reduce to

$$
\begin{aligned}
& O_{1}^{(3)}=-\frac{1}{2 m}\left[u_{\mu},\left[D_{\nu}, u^{\mu}\right]\right] D^{\nu}+\text { h.c. } \longrightarrow \frac{2}{f_{\pi}} \boldsymbol{\tau} \cdot\left(-\frac{1}{f_{\pi}} \partial_{\mu} \boldsymbol{\pi} \times \partial_{0} \partial^{\mu} \boldsymbol{\pi}+\mathbf{A}_{\mu} \times \partial_{0} \partial^{\mu} \boldsymbol{\pi}\right. \\
& \left.-\partial_{0} \mathbf{A}_{\mu} \times \partial^{\mu} \boldsymbol{\pi}\right) \gamma^{0} \\
& O_{2}^{(3)}=-\frac{1}{2 m}\left[u_{\mu},\left[D^{\mu}, u_{\nu}\right]\right] D^{\nu}+\text { h.c. } \longrightarrow \frac{2}{f_{\pi}} \boldsymbol{\tau} \cdot\left(-\frac{1}{f_{\pi}} \partial_{\mu} \boldsymbol{\pi} \times \partial^{\mu} \partial_{0} \boldsymbol{\pi}+\mathbf{A}_{\mu} \times \partial_{0} \partial^{\mu} \boldsymbol{\pi}\right. \\
& \left.-\partial_{\mu} \mathbf{A}_{0} \times \partial^{\mu} \boldsymbol{\pi}\right) \gamma^{0} \\
& O_{3}^{(3)}=\frac{1}{12 m^{3}}\left[u_{\mu},\left[D_{\nu}, u_{\rho}\right]\right] D^{\mu \nu \rho}+\text { h.c. } \longrightarrow \frac{2}{f_{\pi}} \boldsymbol{\tau} \cdot\left(-\frac{1}{f_{\pi}} \partial_{0} \boldsymbol{\pi} \times \partial_{0}^{2} \boldsymbol{\pi}+\mathbf{A}_{0} \times \partial_{0}^{2} \boldsymbol{\pi}\right. \\
& \left.-\partial_{0} \mathbf{A}_{0} \times \partial_{0} \boldsymbol{\pi}\right) \gamma^{0} \\
& O_{4}^{(3)}=-\frac{1}{2 m} \epsilon^{\mu \nu \alpha \beta}\left\langle u_{\mu} u_{\nu} u_{\alpha}\right\rangle D_{\beta}+\text { h.c. } \longrightarrow 0, \\
& O_{5}^{(3)}=\frac{i}{2 m}\left[\chi_{-}, u_{\mu}\right] D^{\mu}+\text { h.c. } \longrightarrow-\frac{4 m_{\pi}^{2}}{f_{\pi}} \boldsymbol{\tau} \cdot\left[\boldsymbol{\pi} \times\left(\frac{1}{f_{\pi}} \partial_{0} \boldsymbol{\pi}-\mathbf{A}_{0}\right)\right] \gamma^{0}, \\
& O_{6}^{(3)}=\frac{i}{2 m}\left[D^{\mu}, \widetilde{F}_{\mu \nu}^{+}\right] D^{\nu}+\text { h.c. } \longrightarrow \partial^{i} F_{i 0}^{+} \gamma^{0} \text {, } \\
& O_{7}^{(3)}=\frac{i}{2 m}\left[D^{\mu},\left\langle F_{\mu \nu}^{+}\right\rangle\right] D^{\nu}+\text { h.c. } \longrightarrow 0, \\
& O_{8}^{(3)}=\frac{i}{2 m} \epsilon^{\mu \nu \alpha \beta}\left\langle\widetilde{F}_{\mu \nu}^{+} u_{\alpha}\right\rangle D_{\beta}+\text { h.c. } \longrightarrow 0, \\
& O_{9}^{(3)}=\frac{i}{2 m} \epsilon^{\mu \nu \alpha \beta}\left\langle F_{\mu \nu}^{+}\right\rangle u_{\alpha} D_{\beta}+\text { h.c. } \longrightarrow 0, \\
& O_{10}^{(3)}=\frac{1}{2} \gamma^{\mu} \gamma_{5}\langle u \cdot u\rangle u_{\mu} \longrightarrow 0 \\
& O_{11}^{(3)}=\frac{1}{2} \gamma^{\mu} \gamma_{5}\left\langle u_{\mu} u_{\nu}\right\rangle u^{\nu} \longrightarrow 0 \\
& O_{12}^{(3)}=-\frac{1}{8 m^{2}} \gamma^{\mu} \gamma_{5}\left\langle u_{\lambda} u_{\nu}\right\rangle u_{\mu} D^{\lambda \nu}+\text { h.c. } \longrightarrow 0 \text {, } \\
& O_{13}^{(3)}=-\frac{1}{8 m^{2}} \gamma^{\mu} \gamma_{5}\left\langle u_{\mu} u_{\nu}\right\rangle u_{\lambda} D^{\lambda \nu}+\text { h.c. } \longrightarrow 0 \text {, } \\
& O_{14}^{(3)}=\frac{i}{4 m} \sigma^{\mu \nu}\left\langle\left[D_{\lambda}, u_{\mu}\right] u_{\nu}\right\rangle D^{\lambda}+\text { h.c. } \longrightarrow \frac{1}{f_{\pi}}\left(\frac{1}{f_{\pi}} \partial_{0} \partial_{i} \boldsymbol{\pi} \cdot \partial_{j} \boldsymbol{\pi}-\partial_{0} \partial_{i} \boldsymbol{\pi} \cdot \mathbf{A}_{j}\right. \\
& \left.-\partial_{0} \mathbf{A}_{i} \cdot \partial_{j} \boldsymbol{\pi}\right) \sigma^{i j} \gamma^{0} \\
& O_{15}^{(3)}=\frac{i}{4 m} \sigma^{\mu \nu}\left\langle u_{\mu}\left[D_{\nu}, u_{\lambda}\right]\right\rangle D^{\lambda}+\text { h.c. } \longrightarrow \frac{1}{f_{\pi}}\left(\frac{1}{f_{\pi}} \partial_{i} \boldsymbol{\pi} \cdot \partial_{0} \partial_{j} \boldsymbol{\pi}-\partial_{i} \boldsymbol{\pi} \cdot \partial_{j} \mathbf{A}_{0}\right. \\
& \left.-\mathbf{A}_{i} \cdot \partial_{0} \partial_{j} \boldsymbol{\pi}\right) \sigma^{i j} \gamma^{0} \\
& O_{16}^{(3)}=\frac{1}{2} \gamma^{\mu} \gamma_{5}\left\langle\chi_{+}\right\rangle u_{\mu} \longrightarrow 2 m_{\pi}^{2} \boldsymbol{\tau} \cdot\left(-\frac{1}{f_{\pi}} \partial_{i} \boldsymbol{\pi}+\mathbf{A}_{i}\right) \gamma^{i} \gamma_{5} \\
& O_{17}^{(3)}=\frac{1}{2} \gamma^{\mu} \gamma_{5}\left\langle\chi_{+} u_{\mu}\right\rangle \longrightarrow 0 \text {, }
\end{aligned}
$$




$$
\begin{aligned}
O_{18}^{(3)} & =\frac{i}{2} \gamma^{\mu} \gamma_{5}\left[D_{\mu}, \chi_{-}\right] \longrightarrow \frac{m_{\pi}^{2}}{f_{\pi}} \boldsymbol{\tau} \cdot \partial_{i} \boldsymbol{\pi} \gamma^{i} \gamma_{5} \\
O_{19}^{(3)} & =\frac{i}{2} \gamma^{\mu} \gamma_{5}\left[D_{\mu},\left\langle\chi_{-}\right\rangle\right] \longrightarrow 0 \\
O_{20}^{(3)} & =-\frac{i}{8 m^{2}} \gamma^{\mu} \gamma_{5}\left[\widetilde{F}_{\mu \nu}^{+}, u_{\lambda}\right] D^{\lambda \nu}+\text { h.c. } \longrightarrow 0 \\
O_{21}^{(3)} & =\frac{i}{2} \gamma^{\mu} \gamma_{5}\left[\widetilde{F}_{\mu \nu}^{+}, u^{\nu}\right] \longrightarrow 0 \\
O_{22}^{(3)} & =\frac{1}{2} \gamma^{\mu} \gamma_{5}\left[D^{\nu}, F_{\mu \nu}^{-}\right] \longrightarrow \frac{1}{2} \boldsymbol{\tau} \cdot \partial^{\nu} \mathbf{F}_{i \nu} \gamma^{i} \gamma_{5}, \\
O_{23}^{(3)} & =\frac{1}{2} \gamma_{\mu} \gamma_{5} \epsilon^{\mu \nu \alpha \beta}\left\langle u_{\nu} F_{\alpha \beta}^{-}\right\rangle \longrightarrow-\frac{1}{f_{\pi}} \epsilon^{i \nu \alpha \beta} \partial_{\nu} \boldsymbol{\pi} \cdot \mathbf{F}_{\alpha \beta} \gamma_{i} \gamma_{5}
\end{aligned}
$$

Several comments are now in order. First, the expressions above for $\mathcal{L}_{\pi N}^{(1)}, \mathcal{L}_{\pi N}^{(2)}$, and $\mathcal{L}_{\pi N}^{(3)}$ retain all terms relevant in the present study. Typically, these include at most three pion, two pion, and one pion fields for $n=1,2,3$ in $\mathcal{L}_{\pi N}^{(n)}$, respectively. In some instances, for example in $O_{1}^{(3)}$, terms with two pion fields are also considered for reasons having to do with the treatment of tadpole-type contributions (see below). The Lagrangian $\sum_{n} \mathcal{L}_{\pi N}^{(n)}$ can now conveniently be expressed as given in Eq. (68) with the quantities $\Gamma_{a}^{0}(n), \Lambda_{a}^{i}(n)$, and $\Delta(n)$, defined in Eqs. (69)-(70), given at leading order by

$$
\begin{aligned}
\Gamma_{a}^{0}(0)= & -\frac{1}{4 f_{\pi}^{2}}(\boldsymbol{\tau} \times \boldsymbol{\pi})_{a} \gamma^{0}+\frac{8 \alpha-1}{16 f_{\pi}^{4}} \boldsymbol{\pi}^{2}(\boldsymbol{\tau} \times \boldsymbol{\pi})_{a} \gamma^{0} \\
\Lambda_{a}^{i}(0)= & -\frac{g_{A}}{2 f_{\pi}}\left(1-\frac{\alpha}{f_{\pi}^{2}} \boldsymbol{\pi}^{2}\right) \tau_{a} \gamma^{i} \gamma_{5}+\frac{g_{A}}{4 f_{\pi}^{3}}(4 \alpha-1)(\boldsymbol{\tau} \cdot \boldsymbol{\pi}) \pi_{a} \gamma^{i} \gamma_{5} \\
\Delta(1)= & \frac{g_{A}}{2} \boldsymbol{\tau} \cdot \mathbf{A}_{i} \gamma^{i} \gamma_{5}+\frac{1}{2 f_{\pi}}\left(1-\frac{\alpha}{f_{\pi}^{2}} \boldsymbol{\pi}^{2}\right)(\boldsymbol{\tau} \times \boldsymbol{\pi}) \cdot \mathbf{A}_{0} \gamma^{0} \\
& +\frac{g_{A}}{4 f_{\pi}^{2}}[(\boldsymbol{\tau} \times \boldsymbol{\pi}) \times \boldsymbol{\pi}] \cdot \mathbf{A}_{i} \gamma^{i} \gamma_{5}
\end{aligned}
$$

at next-to-leading order by

$$
\begin{aligned}
\Gamma_{a}^{0}(1)= & -\frac{g_{A}}{2 f_{\pi}}\left(1-\frac{\alpha}{f_{\pi}^{2}} \boldsymbol{\pi}^{2}\right) \tau_{a} \gamma^{0} \gamma_{5}+\frac{g_{A}}{4 f_{\pi}^{3}}(4 \alpha-1)(\boldsymbol{\tau} \cdot \boldsymbol{\pi}) \pi_{a} \gamma^{0} \gamma_{5}-2 \frac{c_{2}+c_{3}}{f_{\pi}} A_{a}^{0}, \quad(382) \\
\Lambda_{a}^{i}(1)= & -\frac{1}{4 f_{\pi}^{2}}(\boldsymbol{\tau} \times \boldsymbol{\pi})_{a} \gamma^{i}+\frac{c_{3}}{f_{\pi}^{2}} \partial^{i} \pi_{a}-2 \frac{c_{3}}{f_{\pi}} A_{a}^{i}-\frac{c_{4}}{f_{\pi}}\left(\boldsymbol{\tau} \times \mathbf{A}_{j}\right)_{a} \sigma^{i j} \\
& +\frac{c_{4}}{2 f_{\pi}^{2}}\left(\boldsymbol{\tau} \times \partial_{j} \boldsymbol{\pi}\right)_{a} \sigma^{i j}+\frac{(1-8 \alpha)}{8 f_{\pi}^{4}} \boldsymbol{\pi}^{2}(\boldsymbol{\pi} \times \boldsymbol{\tau})_{a} \gamma^{i}, \\
\Delta(2)= & \frac{g_{A}}{2} \boldsymbol{\tau} \cdot \mathbf{A}_{0} \gamma^{0} \gamma^{5}+\frac{1}{2 f_{\pi}}\left(1-\frac{\alpha}{f_{\pi}^{2}} \boldsymbol{\pi}^{2}\right)(\boldsymbol{\tau} \times \boldsymbol{\pi}) \cdot \mathbf{A}_{i} \gamma^{i}+\frac{g_{A}}{4 f_{\pi}^{2}}[(\boldsymbol{\tau} \times \boldsymbol{\pi}) \times \boldsymbol{\pi}] \cdot \mathbf{A}_{0} \gamma^{0} \gamma_{5} \\
& +4 m_{\pi}^{2} c_{1}\left(1-\frac{\boldsymbol{\pi}^{2}}{2 f_{\pi}^{2}}\right)+\frac{c_{6}}{4 m f_{\pi}}(\boldsymbol{\tau} \times \boldsymbol{\pi}) \cdot \partial_{i} \mathbf{A}_{j} \sigma^{i j} ;
\end{aligned}
$$


and at next-to-next-to-leading order by

$$
\begin{aligned}
& \Gamma_{a}^{0}(2)=\frac{c_{2}}{m f_{\pi}}\left(\frac{1}{f_{\pi}} \partial_{i} \pi_{a}-A_{i, a}\right) \gamma^{0} i \overleftrightarrow{\partial}^{i}+\frac{c_{4}}{f_{\pi}}\left[\frac{1}{f_{\pi}}\left(\boldsymbol{\tau} \times \partial_{i} \boldsymbol{\pi}\right)_{a}-\left(\boldsymbol{\tau} \times \mathbf{A}_{i}\right)_{a}\right] \sigma^{0 i} \\
& +2 \frac{d_{1}+d_{2}}{f_{\pi}^{2}}\left[\left(\boldsymbol{\tau} \times \partial^{i} \partial_{i} \boldsymbol{\pi}\right)+\left(\boldsymbol{\tau} \times \partial^{i} \boldsymbol{\pi}\right) \widetilde{\vec{\partial}}_{i}\right] \gamma^{0} \\
& +2 \frac{d_{1}+d_{2}+d_{3}}{f_{\pi}}\left[-\frac{m_{\pi}^{2}}{f_{\pi}}(\boldsymbol{\tau} \times \boldsymbol{\pi})_{a}-\frac{1}{f_{\pi}}\left(\boldsymbol{\tau} \times \partial^{i} \partial_{i} \boldsymbol{\pi}\right)_{a}+\left(\boldsymbol{\tau} \times \partial_{i} \mathbf{A}^{i}\right)_{a}\right] \gamma^{0} \\
& -4 d_{5} \frac{m_{\pi}^{2}}{f_{\pi}^{2}}(\boldsymbol{\tau} \times \boldsymbol{\pi})_{a} \gamma^{0}+\frac{d_{14}-d_{15}}{f_{\pi}}\left[\frac{1}{f_{\pi}} \partial_{i} \pi_{a} \sigma^{i j \overleftrightarrow{\partial}_{j}}+\partial_{i} A_{j, a} \sigma^{i j}\right.
\end{aligned}
$$

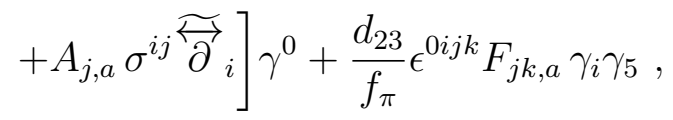

$$
\begin{aligned}
& \Lambda_{a}^{i}(2)=-\frac{c_{2}}{m f_{\pi}} A_{0, a} \gamma^{0} i \overleftrightarrow{\partial}^{i}+\frac{c_{4}}{f_{\pi}}\left(\boldsymbol{\tau} \times \mathbf{A}_{0}\right)_{a} \sigma^{0 i}-2 \frac{d_{1}}{f_{\pi}}\left(\boldsymbol{\tau} \times \partial_{0} \mathbf{A}^{i}\right)_{a} \gamma^{0} \\
& -2 \frac{d_{2}}{f_{\pi}}\left(\boldsymbol{\tau} \times \partial^{i} \mathbf{A}_{0}\right)_{a} \gamma^{0}-\frac{d_{6}}{f_{\pi}}\left(\boldsymbol{\tau} \times \mathbf{F}^{i 0}\right)_{a} \gamma^{0} \\
& +\frac{d_{14}}{f_{\pi}} \partial_{0} A_{j, a} \sigma^{i j} \gamma^{0}-\frac{d_{15}}{f_{\pi}} \partial_{j} A_{0, a} \sigma^{i j} \gamma^{0} \\
& -\frac{m_{\pi}^{2}}{f_{\pi}}\left(2 d_{16}-d_{18}\right) \tau_{a} \gamma^{i} \gamma_{5}+2 \frac{d_{23}}{f_{\pi}} \epsilon^{i j k 0} F_{k 0, a} \gamma_{j} \gamma_{5} \\
& \Delta(3)=\frac{c_{6}}{4 m f_{\pi}}(\boldsymbol{\tau} \times \boldsymbol{\pi}) \cdot\left(\partial_{0} \mathbf{A}_{i}-\partial_{i} \mathbf{A}_{0}\right) \sigma^{0 i}-2 \frac{d_{1}+d_{2}+d_{3}}{f_{\pi}}\left(\boldsymbol{\tau} \times \mathbf{A}_{0}\right) \cdot\left(\partial^{i} \partial_{i} \boldsymbol{\pi}+m_{\pi}^{2} \boldsymbol{\pi}\right) \gamma^{0} \\
& +4 d_{5} \frac{m_{\pi}^{2}}{f_{\pi}} \boldsymbol{\tau} \cdot\left(\boldsymbol{\pi} \times \mathbf{A}_{0}\right) \gamma^{0}+\frac{d_{6}}{f_{\pi}}(\boldsymbol{\tau} \times \boldsymbol{\pi}) \cdot \partial^{i} \mathbf{F}_{i 0} \gamma^{0}+2 m_{\pi}^{2} d_{16} \boldsymbol{\tau} \cdot \mathbf{A}_{i} \gamma^{i} \gamma_{5} \\
& +\frac{d_{22}}{2} \boldsymbol{\tau} \cdot \partial^{\nu} \mathbf{F}_{i \nu} \gamma^{i} \gamma_{5}
\end{aligned}
$$

Second, the various derivatives act only on the field to their immediate right, for example $\partial_{0} \boldsymbol{\pi} \cdot \mathbf{A}_{0}$ means $\left(\partial_{0} \boldsymbol{\pi}\right) \cdot \mathbf{A}_{0}$. However, the symbols $\overleftrightarrow{\partial}_{i}=\vec{\partial}_{i}-\overleftarrow{\partial}_{i}$ and $\overleftrightarrow{\partial}_{i}=\vec{\partial}_{i}+\overleftarrow{\partial}_{i}$ in Eqs. (349) and (385)-(386) denote derivatives acting only on the right and left nucleon fields, respectively.

Third, the power counting $Q^{n}$ of $\mathcal{L}_{\pi N}^{(n)}$ counts powers of derivatives of the pion field (or of pion mass factors) and factors of $A_{a}^{\mu}$ and its derivatives (note that $A_{a}^{\mu}$ is counted as being of order $Q$ ). However, the Lorentz structure of the terms may lead to additional suppression. For example, in $\mathcal{L}_{\pi N}^{(1)}$ a term like

$$
-\frac{1}{4 f_{\pi}^{2}}(\boldsymbol{\tau} \times \boldsymbol{\pi}) \cdot \partial_{0} \boldsymbol{\pi} \gamma^{0}
$$


is of order $Q$, but a term like

$$
-\frac{g_{A}}{2 f_{\pi}}\left(1-\frac{\alpha}{f_{\pi}^{2}} \boldsymbol{\pi}^{2}\right) \boldsymbol{\tau} \cdot \partial_{0} \boldsymbol{\pi} \gamma^{0} \gamma_{5}
$$

which is nominally of order $Q$, is in fact of order $Q^{2}$, since $\bar{N} \gamma^{0} \gamma_{5} N$ couples the lower to the upper components of the spinors, and therefore involves the three-momenta of the initial and final nucleons (of order $Q$ ). We have taken advantage of this suppression in some of the terms $O_{i}^{(3)}$ in $\mathcal{L}_{\pi N}^{(3)}$ by retaining only the diagonal piece in their Lorentz structure, for example in term $O_{14}^{(3)}$.

Fourth, time derivatives of the nucleon fields in $\mathcal{L}_{\pi N}^{(2)}$ and $\mathcal{L}_{\pi N}^{(3)}$ are removed by making use of the equation of motion (to order $Q$ )

$$
\partial_{0} N=-i m \gamma^{0} N+\left[-\gamma^{0} \gamma^{i} \partial_{i}+i \gamma^{0} \Gamma_{a}^{0}(0) \partial_{0} \pi_{a}+i \gamma^{0} \Lambda_{a}^{i}(0) \partial_{i} \pi_{a}+i \gamma^{0} \Delta(1)\right] N,
$$

implying that

$$
\begin{aligned}
\partial_{0}^{2} N & =-m^{2} N-i m \gamma^{0}[\ldots] N-i m[\ldots] \gamma^{0} N \\
& =-m^{2} N+\left[-\frac{m g_{A}}{f_{\pi}} \boldsymbol{\tau} \cdot \partial_{i} \boldsymbol{\pi} \gamma^{i} \gamma^{5}+m g_{A} \boldsymbol{\tau} \cdot \mathbf{A}_{i} \gamma^{i} \gamma_{5}-\frac{m}{f_{\pi}} \boldsymbol{\tau} \cdot\left(\mathbf{A}_{0} \times \boldsymbol{\pi}\right) \gamma^{0}\right] N,
\end{aligned}
$$

where in the second line we have ignored non-linear terms in the pion field, since they do not contribute to the order of interest here.

Fifth, double time derivatives of the pion fields in $\mathcal{L}_{\pi N}^{(3)}$ are removed by making use of the equation of motion, see Eq. (343) above. Terms containing both one time derivative and one space derivative of the pion fields have been rewritten by integrating by parts. For example, in $\mathcal{L}_{\pi N}^{(3)}$ a term like

$$
2 \frac{d_{1}+d_{2}}{f_{\pi}^{2}} \bar{N}\left(\boldsymbol{\tau} \times \partial_{0} \partial^{i} \boldsymbol{\pi}\right) \cdot \partial_{i} \boldsymbol{\pi} N
$$

can be re-expressed, modulo a total divergence, as

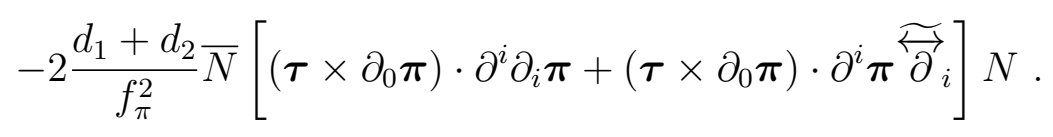




\section{APPENDIX B}

\section{INTERACTION VERTICES}

In this appendix we report expressions for the vertices corresponding to the interaction terms in the Hamiltonian of Eq. (75), which we write as

$$
\begin{aligned}
H_{I}= & \sum_{n=1}^{3}\left[\left(H_{\pi N N}^{(n)}+H_{2 \pi N N}^{(n)}+H_{3 \pi N N}^{(n)}+\cdots\right)+\left(H_{N N A}^{(n)}+H_{\pi N N A}^{(n)}+H_{2 \pi N N A}^{(n)}+\cdots\right)\right] \\
& +\sum_{m=1}^{2}\left[\left(H_{2 \pi}^{(2 m)}+H_{4 \pi}^{(2 m)}+\cdots\right)+\left(H_{\pi A}^{(2 m)}+H_{3 \pi A}^{(2 m)}+\cdots\right)\right]
\end{aligned}
$$

where the superscript $n$ denotes the power counting $Q^{n}$ and the subscript specifies the number of pion, nucleon, and axial fields entering a given interaction term. We use the following notation: $\lambda=\mathbf{p} \sigma \tau\left(\lambda^{\prime}=\mathbf{p}^{\prime} \sigma^{\prime} \tau^{\prime}\right)$ are the momentum and spin and isospin projections of the initial (final) nucleon; $\mathbf{k}_{1}, \mathbf{k}_{2}, \ldots$ and $a_{1}, a_{2}, \ldots$ are the momenta and isospin projections of pions $1,2, \ldots$ with energies $\omega_{1}, \omega_{2}, \ldots$, where $\omega_{i}=\sqrt{k_{i}^{2}+m_{\pi}^{2}} ; \mathbf{q}$ and $a$ denote the momentum and isospin projection of the external axial field with energy $\omega_{q}$ and its spatial and time derivatives expressed as $\boldsymbol{\nabla} A_{a}^{\mu} \longrightarrow i \mathbf{q} A_{a}^{\mu}$ and $\partial_{0} A_{a}^{\mu} \longrightarrow-i \omega_{q} A_{a}^{\mu}$. We also define $\mathbf{P}=$ $\left(\mathbf{p}^{\prime}+\mathbf{p}\right) / 2$ and the constants

$$
J_{m n}=\int \frac{\mathrm{d} \mathbf{l}}{(2 \pi)^{3}} \frac{l^{2 m}}{\omega_{l}^{n}} .
$$

\section{B.1 $\pi N N$ VERTICES}

The interaction terms read

$$
\begin{aligned}
H_{\pi N N}^{(1)} & =\frac{g_{A}}{2 f_{\pi}} \int \mathrm{d} \mathbf{x} \bar{N} \boldsymbol{\tau} \cdot \partial_{i} \boldsymbol{\pi} \gamma^{i} \gamma^{5} N \\
H_{\pi N N}^{(2)} & =\frac{g_{A}}{2 f_{\pi}} \int \mathrm{d} \mathbf{x} \bar{N} \boldsymbol{\tau} \cdot \Pi \mathbf{\Pi} \gamma^{0} \gamma^{5} N \\
H_{\pi N N}^{(3)} & =\frac{m_{\pi}^{2}}{f_{\pi}}\left(2 d_{16}-d_{18}\right) \int \mathrm{d} \mathbf{x} \bar{N} \boldsymbol{\tau} \cdot \partial_{i} \boldsymbol{\pi} \gamma^{i} \gamma^{5} N
\end{aligned}
$$


from which the following vertices for pion absorption are obtained

$$
\begin{aligned}
\left\langle\lambda^{\prime}\left|H_{\pi N N}^{(1)}\right| \lambda ; \mathbf{k}, a\right\rangle= & i \frac{g_{A}}{2 f_{\pi}} \tau_{a} \boldsymbol{\sigma} \cdot \mathbf{k} \\
\left\langle\lambda^{\prime}\left|H_{\pi N N}^{(2)}\right| \lambda ; \mathbf{k}, a\right\rangle= & -i \frac{g_{A}}{2 m f_{\pi}} \tau_{a} \omega \boldsymbol{\sigma} \cdot \mathbf{P}, \\
\left\langle\lambda^{\prime}\left|H_{\pi N N}^{(3)}\right| \lambda ; \mathbf{k}, a\right\rangle= & i \frac{m_{\pi}^{2}}{f_{\pi}}\left(2 d_{16}-d_{18}\right) \tau_{a} \boldsymbol{\sigma} \cdot \mathbf{k} \\
& +i \frac{g_{A}}{8 m^{2} f_{\pi}} \tau_{a}\left[2 \boldsymbol{\sigma} \cdot \mathbf{P} \mathbf{k} \cdot \mathbf{P}-\boldsymbol{\sigma} \cdot\left(\mathbf{p}^{\prime}-\mathbf{p}\right) \frac{\left(\mathbf{p}^{\prime}-\mathbf{p}\right) \cdot \mathbf{k}}{2}\right. \\
& \left.-2 P^{2} \boldsymbol{\sigma} \cdot \mathbf{k}-i \mathbf{k} \cdot\left(\mathbf{p}^{\prime}-\mathbf{p}\right) \times \mathbf{P}\right],
\end{aligned}
$$

where on the r.h.s. of the above equations the $1 / \sqrt{2 \omega}$ normalization factor from the pion field expansion in normal modes, the initial and final spin-isospin states of the nucleons, and the three-momentum conserving $\delta$-function $(2 \pi)^{3} \delta\left(\mathbf{p}^{\prime}-\mathbf{p}-\mathbf{k}\right)$ have been dropped for simplicity. We will continue to do so in the equations to follow. Vertices in which the pion is in the final state (pion emission) are obtained from those above by the replacements $\omega, \mathbf{k} \longrightarrow-\omega,-\mathbf{k}$. Lastly, only the leading order is retained in the non-relativistic expansion of the Lorentz structures associated with the various interaction terms (here and to follow) unless otherwise noted. Indeed, Eq. (398) includes the leading relativistic correction to the vertex given in Eq. (396).

\section{B.2 $2 \pi N N$ VERTICES}

The interaction terms read

$$
\begin{aligned}
H_{2 \pi N N}^{(1)}= & \frac{1}{4 f_{\pi}^{2}} \int \mathrm{d} \mathbf{x} \bar{N} \boldsymbol{\Pi} \cdot(\boldsymbol{\tau} \times \boldsymbol{\pi}) \gamma^{0} N, \\
H_{2 \pi N N}^{(2)}= & \int \mathrm{d} \mathbf{x} \bar{N}\left[\frac{1}{4 f_{\pi}^{2}} \partial_{i} \boldsymbol{\pi} \cdot(\boldsymbol{\tau} \times \boldsymbol{\pi}) \gamma^{i}+c_{1} \frac{2 m_{\pi}^{2}}{f_{\pi}^{2}} \boldsymbol{\pi}^{2}-\frac{c_{3}}{f_{\pi}^{2}} \partial^{i} \boldsymbol{\pi} \cdot \partial_{i} \boldsymbol{\pi}+\right. \\
& \left.-\frac{c_{2}+c_{3}}{f_{\pi}^{2}} \boldsymbol{\Pi} \cdot \boldsymbol{\Pi}+\frac{c_{4}}{2 f_{\pi}^{2}} \boldsymbol{\tau} \cdot\left(\partial_{i} \boldsymbol{\pi} \times \partial_{j} \boldsymbol{\pi}\right) \sigma^{i j}\right] N, \\
H_{2 \pi N N}^{(3)}= & \int \mathrm{d} \mathbf{x} \bar{N}\left[-2 \frac{d_{1}+d_{2}+d_{3}}{f_{\pi}^{2}}(\boldsymbol{\tau} \times \boldsymbol{\Pi}) \cdot\left(\partial^{i} \partial_{i} \boldsymbol{\pi}+m_{\pi}^{2} \boldsymbol{\pi}\right) \gamma^{0}-4 \frac{d_{5} m_{\pi}^{2}}{f_{\pi}^{2}}(\boldsymbol{\Pi} \times \boldsymbol{\pi}) \cdot \boldsymbol{\tau} \gamma^{0}\right. \\
& \left.+2 \frac{d_{1}+d_{2}}{f_{\pi}^{2}}(\boldsymbol{\tau} \times \boldsymbol{\Pi}) \cdot\left(\partial^{i} \partial_{i} \boldsymbol{\pi}+\partial^{i} \boldsymbol{\pi} \overleftrightarrow{\mathrm{\partial}}_{i}\right) \gamma^{0}+\frac{d_{15}-d_{14}}{f_{\pi}^{2}} \boldsymbol{\Pi} \cdot \partial_{i} \boldsymbol{\pi} \sigma^{i j}{\overleftrightarrow{\partial_{j}}}_{j}^{0}\right] N,
\end{aligned}
$$


from which the vertices follow as

$$
\begin{aligned}
\left\langle\lambda^{\prime}\left|H_{2 \pi N N}^{(1)}\right| \lambda ; \mathbf{k}_{1}, a_{1} ; \mathbf{k}_{2}, a_{2}\right\rangle= & \frac{i}{4 f_{\pi}^{2}} \epsilon_{a_{1} a_{2} c} \tau_{c}\left(\omega_{1}-\omega_{2}\right) \\
\left\langle\lambda^{\prime}\left|H_{2 \pi N N}^{(2)}\right| \lambda ; \mathbf{k}_{1}, a_{1} ; \mathbf{k}_{2}, a_{2}\right\rangle= & -\frac{i}{4 f_{\pi}^{2}} \frac{2 \mathbf{P}+i \boldsymbol{\sigma} \times\left(\mathbf{p}^{\prime}-\mathbf{p}\right)}{2 m} \cdot\left(\mathbf{k}_{1}-\mathbf{k}_{2}\right) \epsilon_{a_{1} a_{2} a} \tau_{a}+4 c_{1} \frac{m_{\pi}^{2}}{f_{\pi}^{2}} \delta_{a_{1}, a_{2}} \\
& -\frac{2 c_{3}}{f_{\pi}^{2}} \mathbf{k}_{1} \cdot \mathbf{k}_{2} \delta_{a_{1}, a_{2}}+\frac{2\left(c_{2}+c_{3}\right)}{f_{\pi}^{2}} \omega_{1} \omega_{2} \delta_{a_{1}, a_{2}} \\
& -\frac{c_{4}}{f_{\pi}^{2}} \boldsymbol{\sigma} \cdot\left(\mathbf{k}_{1} \times \mathbf{k}_{2}\right) \epsilon_{a_{1} a_{2} a} \tau_{a} \\
\left\langle\lambda^{\prime}\left|H_{2 \pi N N}^{(3)}\right| \lambda ; \mathbf{k}_{1}, a_{1} ; \mathbf{k}_{2}, a_{2}\right\rangle= & i\left(\omega_{1}-\omega_{2}\right)\left[\epsilon _ { a _ { 1 } a _ { 2 } c } \tau _ { c } \left(-2 \frac{d_{1}+d_{2}+d_{3}}{f_{\pi}^{2}} \omega_{1} \omega_{2}+4 \frac{d_{5} m_{\pi}^{2}}{f_{\pi}^{2}}\right.\right. \\
& \left.\left.+2 \frac{d_{1}+d_{2}}{f_{\pi}^{2}} \mathbf{k}_{1} \cdot \mathbf{k}_{2}\right)+\frac{d_{15}-d_{14}}{f_{\pi}^{2}}\left(\mathbf{k}_{1} \times \mathbf{k}_{2}\right) \cdot \boldsymbol{\sigma} \delta_{a_{1}, a_{2}}\right]
\end{aligned}
$$

Vertices in which either or both pions are in the final state are obtained from those above by replacing $\mathbf{k}_{i}, \omega_{i} \longrightarrow-\mathbf{k}_{i},-\omega_{i}$.

\section{B.3 $3 \pi N N$ VERTEX}

The interaction term reads

$$
H_{3 \pi N N}^{(1)}=-\frac{g_{A}}{2 f_{\pi}^{3}} \int \mathrm{d} \mathbf{x} \bar{N}\left[\alpha \boldsymbol{\pi}^{2} \boldsymbol{\tau} \cdot \partial_{i} \boldsymbol{\pi}+\frac{1}{2}(4 \alpha-1) \boldsymbol{\tau} \cdot \boldsymbol{\pi} \boldsymbol{\pi} \cdot \partial_{i} \boldsymbol{\pi}\right] \gamma^{i} \gamma^{5} N,
$$

which leads to the following interaction vertex

$$
\begin{aligned}
\left\langle\lambda^{\prime}\left|H_{3 \pi N N}^{(1)}\right| \lambda ; \mathbf{k}_{1}, a_{1} ; \mathbf{k}_{2}, a_{2} ; \mathbf{k}_{3}, a_{3}\right\rangle= & -\frac{i g_{A}}{2 f_{\pi}^{3}} \boldsymbol{\sigma} \cdot\left[\tau_{a_{1}} \delta_{a_{2}, a_{3}}\left[(2 \alpha-1 / 2)\left(\mathbf{k}_{2}+\mathbf{k}_{3}\right)+2 \alpha \mathbf{k}_{1}\right]\right. \\
& +\tau_{a_{2}} \delta_{a_{1}, a_{3}}\left[(2 \alpha-1 / 2)\left(\mathbf{k}_{1}+\mathbf{k}_{3}\right)+2 \alpha \mathbf{k}_{2}\right] \\
& \left.+\tau_{a_{3}} \delta_{a_{1}, a_{2}}\left[(2 \alpha-1 / 2)\left(\mathbf{k}_{1}+\mathbf{k}_{2}\right)+2 \alpha \mathbf{k}_{3}\right]\right] . \quad(406)
\end{aligned}
$$

The corresponding tadpole contribution is

$$
\left\langle\lambda^{\prime}\left|H_{3 \pi N N}^{(1)}\right| \lambda ; \mathbf{k}, a\right\rangle=-i \frac{g_{A}}{8 f_{\pi}^{3}}(10 \alpha-1) J_{01} \tau_{a} \boldsymbol{\sigma} \cdot \mathbf{k}
$$

where $J_{01}$ has been defined in Eq. (392).

\section{B.4 $4 \pi N N$ VERTEX}

The interaction term reads

$$
H_{4 \pi N N}^{(1)}=\frac{1}{32 f_{\pi}^{4}} \int \mathrm{d} \mathbf{x} \bar{N}\left(\Pi_{a} \boldsymbol{\pi}^{2}+\boldsymbol{\pi}^{2} \Pi_{a}\right)(\boldsymbol{\tau} \times \boldsymbol{\pi})_{a} \gamma^{0} N,
$$


and the tadpole contribution follows as

$$
\left\langle 0\left|H_{4 \pi N N}^{(1)}\right| \mathbf{k}_{1}, a_{1} ; \mathbf{k}_{2}, a_{2}\right\rangle=\frac{5 i}{32 f_{\pi}^{4}} J_{01} \epsilon_{a_{1} a_{2} c} \tau_{c}\left(\omega_{1}-\omega_{2}\right) .
$$

\section{B.5 NNA VERTICES}

The interaction terms read

$$
\begin{aligned}
H_{N N A}^{(1)} & =-\frac{g_{A}}{2} \int \mathrm{d} \mathbf{x} \bar{N} \tau_{a} A_{a}^{i} \gamma_{i} \gamma^{5} N \\
H_{N N A}^{(3)} & =-\int \mathrm{d} \mathbf{x} \bar{N}\left(2 m_{\pi}^{2} d_{16} \boldsymbol{\tau} \cdot \mathbf{A}_{i} \gamma^{i} \gamma_{5}+\frac{d_{22}}{2} \boldsymbol{\tau} \cdot \partial^{j} \mathbf{F}_{i j} \gamma^{i} \gamma_{5}\right) N
\end{aligned}
$$

from which the vertices follow as

$$
\begin{aligned}
\left\langle\lambda^{\prime}\left|H_{N N A}^{(1)}\right| \lambda\right\rangle= & \frac{g_{A}}{2} \tau_{a}\left[\boldsymbol{\sigma}-\frac{1}{2 m^{2}} P^{2} \boldsymbol{\sigma}-\frac{i}{4 m^{2}}\left(\mathbf{p}^{\prime}-\mathbf{p}\right) \times \mathbf{P}+\frac{1}{2 m^{2}} \boldsymbol{\sigma} \cdot \mathbf{P} \mathbf{P}\right. \\
& \left.-\frac{1}{8 m^{2}} \boldsymbol{\sigma} \cdot\left(\mathbf{p}^{\prime}-\mathbf{p}\right)\left(\mathbf{p}^{\prime}-\mathbf{p}\right)\right] \cdot \mathbf{A}_{a}, \\
\left\langle\lambda^{\prime}\left|H_{N N A}^{(3)}\right| \lambda\right\rangle= & 2 m_{\pi}^{2} d_{16} \tau_{a} \boldsymbol{\sigma} \cdot \mathbf{A}_{a}+\frac{d_{22}}{2} \tau_{a}\left(\mathbf{q} \mathbf{q} \cdot \boldsymbol{\sigma}-q^{2} \boldsymbol{\sigma}\right) \cdot \mathbf{A}_{a},
\end{aligned}
$$

where in Eq. (413) terms of order $Q^{2}$ have been retained in the expansion of the bilinear $\bar{N} \gamma \gamma_{5} N$, since they have been shown to generate significant corrections to the single-nucleon axial current [48].

\section{B.6 $\pi N N A$ VERTICES}

The interaction terms read

$$
\begin{aligned}
& H_{\pi N N A}^{(1)}=-\frac{1}{4 f_{\pi}} \int \mathrm{d} \mathbf{x} \bar{N} \mathbf{A}_{0} \cdot(\boldsymbol{\tau} \times \boldsymbol{\pi}) \gamma^{0} N \\
& H_{\pi N N A}^{(2)}=\int \mathrm{d} \mathbf{x} \bar{N}\left[-\frac{1}{2 f_{\pi}}(\boldsymbol{\tau} \times \boldsymbol{\pi}) \cdot \mathbf{A}_{i} \gamma^{i}-\frac{c_{6}}{4 m f_{\pi}}(\boldsymbol{\tau} \times \boldsymbol{\pi}) \cdot \partial_{i} \mathbf{A}_{j} \sigma^{i j}+\frac{2 c_{3}}{f_{\pi}} \mathbf{A}^{i} \cdot \partial_{i} \boldsymbol{\pi}\right. \\
& \left.+\frac{c_{4}}{f_{\pi}}\left(\partial_{i} \boldsymbol{\pi} \times \boldsymbol{\tau}\right) \cdot \mathbf{A}_{j} \sigma^{i j}\right] N \\
& H_{\pi N N A}^{(3)}=\int \mathrm{d} \mathbf{x} \bar{N}\left[\frac{2 d_{2}+d_{6}}{f_{\pi}}\left(\partial_{i} \boldsymbol{\pi} \times \boldsymbol{\tau}\right) \cdot \partial^{i} \mathbf{A}^{0} \gamma^{0}+\frac{d_{15}}{f_{\pi}} \partial_{j} \mathbf{A}^{0} \cdot \partial_{i} \boldsymbol{\pi} \sigma^{i j} \gamma^{0}\right. \\
& +2 \frac{d_{23}}{f_{\pi}} \epsilon^{0 i j k} \partial_{i} \boldsymbol{\pi} \cdot \partial_{k} \mathbf{A}^{0} \gamma_{j} \gamma^{5}-\frac{d_{6}}{f_{\pi}}(\boldsymbol{\tau} \times \boldsymbol{\pi}) \cdot \partial_{i} \partial^{i} \mathbf{A}^{0} \gamma^{0} \\
& \left.+2 \frac{d_{1}+d_{2}}{f_{\pi}}\left(\boldsymbol{\tau} \times \mathbf{A}_{0}\right) \cdot\left(\partial^{i} \partial_{i} \boldsymbol{\pi}+\partial^{i} \boldsymbol{\pi} \widetilde{\vec{\partial}}_{i}\right) \gamma^{0}+\frac{d_{15}-d_{14}}{f_{\pi}} \partial_{i} \boldsymbol{\pi} \cdot \mathbf{A}_{0} \sigma^{i j} \widetilde{\overleftrightarrow{\partial}}_{j}+\ldots\right] N
\end{aligned}
$$


where the dots indicate terms which do not contribute in tree-level diagrams of order $Q$, for example

$$
\int \mathrm{d} \mathbf{x} \bar{N}\left[-2 \frac{d_{23}}{f_{\pi}} \epsilon^{0 i j k} \gamma_{i} \gamma^{5} \boldsymbol{\Pi} \cdot \partial_{j} \mathbf{A}_{k}-2 \frac{d_{1}+d_{2}+d_{3}}{f_{\pi}} \boldsymbol{\tau} \cdot\left(\partial_{i} \mathbf{A}^{i} \times \mathbf{\Pi}\right) \gamma^{0}\right] N
$$

or

$$
2 \frac{d_{1}+d_{2}+d_{3}}{f_{\pi}} \int \mathrm{d} \mathbf{x} \bar{N} \boldsymbol{\tau} \cdot\left(\partial_{0} \mathbf{A}_{0} \times \mathbf{\Pi}\right) \gamma^{0} N
$$

and $\partial_{0} \mathbf{A}_{0} \rightarrow-i \omega_{q} \mathbf{A}_{0}$ is of order $Q^{3}$, since in our counting the energy of the external field is of order $Q^{2}$. The interactions in Eqs. (414)-(416) lead to the following vertices

$$
\begin{aligned}
\left\langle\lambda^{\prime}\left|H_{\pi N N A}^{(1)}\right| \lambda ; \mathbf{k}, a\right\rangle= & -\frac{1}{4 f_{\pi}} \epsilon_{a b c} A_{b}^{0} \tau_{c} \\
\left\langle\lambda^{\prime}\left|H_{\pi N N A}^{(2)}\right| \lambda ; \mathbf{k}, a\right\rangle= & -\frac{1}{2 m f_{\pi}} \epsilon_{a b c} \tau_{b} \mathbf{A}_{c} \cdot\left[\mathbf{P}+\frac{i}{2} \boldsymbol{\sigma} \times\left(\mathbf{p}^{\prime}-\mathbf{p}\right)\right] \\
& -i \frac{c_{6}}{4 m f_{\pi}} \epsilon_{a b c} \tau_{b} \mathbf{A}_{c} \cdot(\boldsymbol{\sigma} \times \mathbf{q})+2 i \frac{c_{3}}{f_{\pi}} \mathbf{k} \cdot \mathbf{A}_{a} \\
& -i \frac{c_{4}}{f_{\pi}} \epsilon_{a b c} \tau_{b} \mathbf{A}_{c} \cdot(\boldsymbol{\sigma} \times \mathbf{k}) \\
\left\langle\lambda^{\prime}\left|H_{\pi N N A}^{(3)}\right| \lambda ; \mathbf{k}, a\right\rangle= & \frac{2 d_{1}-d_{6}}{f_{\pi}}\left(\mathbf{A}^{0} \times \boldsymbol{\tau}\right)_{a} \mathbf{q} \cdot \mathbf{k}+\frac{d_{14}+2 d_{23}}{f_{\pi}} \boldsymbol{\sigma} \cdot(\mathbf{q} \times \mathbf{k}) A_{a}^{0} \\
& -\frac{d_{6}}{f_{\pi}}\left(\mathbf{A}^{0} \times \boldsymbol{\tau}\right)_{a} \mathbf{q}^{2}
\end{aligned}
$$

\section{B.7 $2 \pi N N A$ VERTICES}

The interaction term reads

$$
H_{2 \pi N N A}^{(1)}=-\frac{g_{A}}{4 f_{\pi}^{2}} \int \mathrm{d} \mathbf{x} \bar{N} \mathbf{A}_{i} \cdot[(\boldsymbol{\tau} \times \boldsymbol{\pi}) \times \boldsymbol{\pi}] \gamma^{i} \gamma^{5} N
$$

which leads to the following vertex and tadpole contributions

$$
\begin{aligned}
\left\langle\lambda^{\prime}\left|H_{2 \pi N N A}^{(1)}\right| \lambda ; \mathbf{k}_{1}, a_{1} ; \mathbf{k}_{2}, a_{2}\right\rangle & =\frac{g_{A}}{4 f_{\pi}^{2}}\left(\delta_{a, a_{1}} \tau_{a_{2}}+\delta_{a, a_{2}} \tau_{a_{1}}-2 \delta_{a_{1}, a_{2}} \tau_{a}\right) \mathbf{A}_{a} \cdot \boldsymbol{\sigma} \\
\left\langle\lambda^{\prime}\left|H_{2 \pi N N A}^{(1)}\right| \lambda\right\rangle & =-\frac{g_{A}}{4 f_{\pi}^{2}} J_{01} \tau_{a} \mathbf{A}_{a} \cdot \boldsymbol{\sigma}
\end{aligned}
$$

\section{B.8 $3 \pi N N A$ VERTICES}

The interaction term reads

$$
H_{3 \pi N N A}^{(1)}=\frac{4 \alpha-1}{16 f_{\pi}^{3}} \int d \mathbf{x} \bar{N} \boldsymbol{\pi}^{2} \mathbf{A}^{0} \cdot(\boldsymbol{\tau} \times \boldsymbol{\pi}) \gamma^{0} N,
$$


from which the tadpole contribution is obtained as

$$
\left\langle\lambda^{\prime}\left|H_{3 \pi N N A}^{(1)}\right| \lambda ; \mathbf{k}, a\right\rangle=-\frac{5(4 \alpha-1)}{32 f_{\pi}^{3}} J_{01}\left(\boldsymbol{\tau} \times \mathbf{A}^{0}\right)_{a} .
$$

\section{B.9 $2 \pi$ VERTICES}

The interaction terms read

$$
H_{2 \pi}^{(4)}=\int \mathrm{d} \mathbf{x}\left[-\frac{m_{\pi}^{2} l_{4}}{f_{\pi}^{2}}\left(\boldsymbol{\Pi} \cdot \boldsymbol{\Pi}+\partial^{i} \boldsymbol{\pi} \cdot \partial_{i} \boldsymbol{\pi}\right)+\frac{m_{\pi}^{4}\left(l_{3}+l_{4}\right)}{f_{\pi}^{2}} \boldsymbol{\pi} \cdot \boldsymbol{\pi}\right],
$$

from which the vertex is obtained as

$$
\left\langle 0\left|H_{2 \pi}^{(4)}\right| \mathbf{k}_{1}, a_{1} ; \mathbf{k}_{2}, a_{2}\right\rangle=\delta_{a_{1}, a_{2}}\left[\frac{2 m_{\pi}^{2} l_{4}}{f_{\pi}^{2}}\left(\omega_{1} \omega_{2}-\mathbf{k}_{1} \cdot \mathbf{k}_{2}\right)+\frac{2 m_{\pi}^{4}\left(l_{3}+l_{4}\right)}{f_{\pi}^{2}}\right]
$$

where, as noted earlier, the momentum-conserving $\delta$-function $(2 \pi)^{3} \delta\left(\mathbf{k}_{1}+\mathbf{k}_{2}\right)$ and the pion field normalization factor $1 / \sqrt{4 \omega_{1} \omega_{2}}$ are understood. Vertices in which one or both pions are in the final state follow by replacing $\omega_{i}, \mathbf{k}_{i} \longrightarrow-\omega_{i},-\mathbf{k}_{i}$. Enforcing the $\delta$ function requirement $\mathbf{k}_{1}=-\mathbf{k}_{2}=\mathbf{k}$ and $\omega_{1}=\omega_{2}=\omega$, the vertex in Eq. (426) reduces to

$$
\left\langle 0\left|H_{2 \pi}^{(4)}\right| \mathbf{k}, a ;-\mathbf{k}, a\right\rangle=\frac{4 m_{\pi}^{2} l_{4}}{f_{\pi}^{2}} \omega^{2}+\frac{2 m_{\pi}^{4} l_{3}}{f_{\pi}^{2}} .
$$

Similarly, we find

$$
\left\langle\mathbf{k}, a\left|H_{2 \pi}^{(4)}\right| \mathbf{k}, a\right\rangle=\frac{2 m_{\pi}^{4} l_{3}}{f_{\pi}^{2}}
$$

according to the prescription given above. Apart from the factor $1 /(2 \omega)$, which is not included in the equations above, these vertices are the same as given in Appendix $\mathrm{F}$ of Ref. [47].

\section{B.10 $4 \pi$ VERTICES}

The interaction terms read

$$
\begin{aligned}
H_{4 \pi}^{(2)}= & \int d \mathbf{x}\left[\frac{4 \alpha-1}{2 f_{\pi}^{2}}\left(\boldsymbol{\pi} \cdot \boldsymbol{\Pi} \boldsymbol{\Pi} \cdot \boldsymbol{\pi}+\partial_{i} \boldsymbol{\pi} \cdot \boldsymbol{\pi} \partial^{i} \boldsymbol{\pi} \cdot \boldsymbol{\pi}\right)\right. \\
& \left.+\frac{\alpha}{f_{\pi}^{2}}\left(\pi_{a} \boldsymbol{\Pi} \cdot \boldsymbol{\Pi} \pi_{a}+\boldsymbol{\pi}^{2} \partial_{i} \boldsymbol{\pi} \cdot \partial^{i} \boldsymbol{\pi}\right)-\frac{8 \alpha-1}{8 f_{\pi}^{2}} m_{\pi}^{2} \boldsymbol{\pi}^{4}\right]
\end{aligned}
$$


which leads to the following vertex

$$
\begin{aligned}
& \left\langle 0\left|H_{4 \pi}^{(2)}\right| \mathbf{k}_{1}, a_{1} ; \mathbf{k}_{2}, a_{2} ; \mathbf{k}_{3}, a_{3} ; \mathbf{k}_{4}, a_{4}\right\rangle=\frac{1}{f_{\pi}^{2}} \\
& \times\left[\delta_{a_{1}, a_{2}} \delta_{a_{3}, a_{4}}\left[-2 \alpha\left(\omega_{1}+\omega_{2}+\omega_{3}+\omega_{4}\right)^{2}+m_{\pi}^{2}+\left(\mathbf{k}_{3}+\mathbf{k}_{4}\right)^{2}+\left(\omega_{1}+\omega_{2}\right)\left(\omega_{3}+\omega_{4}\right)\right]\right. \\
& +\delta_{a_{1}, a_{3}} \delta_{a_{2}, a_{4}}\left[-2 \alpha\left(\omega_{1}+\omega_{2}+\omega_{3}+\omega_{4}\right)^{2}+m_{\pi}^{2}+\left(\mathbf{k}_{1}+\mathbf{k}_{3}\right)^{2}+\left(\omega_{1}+\omega_{3}\right)\left(\omega_{2}+\omega_{4}\right)\right] \\
& \left.+\delta_{a_{1}, a_{4}} \delta_{a_{2}, a_{3}}\left[-2 \alpha\left(\omega_{1}+\omega_{2}+\omega_{3}+\omega_{4}\right)^{2}+m_{\pi}^{2}+\left(\mathbf{k}_{1}+\mathbf{k}_{4}\right)^{2}+\left(\omega_{1}+\omega_{4}\right)\left(\omega_{2}+\omega_{3}\right)\right]\right],
\end{aligned}
$$

and the corresponding tadpole contribution is

$$
\left\langle 0\left|H_{4 \pi}^{(2)}\right| \mathbf{k}_{1}, a_{1} ; \mathbf{k}_{2}, a_{2}\right\rangle=\delta_{a_{1}, a_{2}} J_{01}\left[\frac{1-10 \alpha}{2 f_{\pi}^{2}}\left(\omega_{1} \omega_{2}-\mathbf{k}_{1} \cdot \mathbf{k}_{2}\right)-\frac{20 \alpha-3}{4 f_{\pi}^{2}} m_{\pi}^{2}\right] \text {, }
$$

and the constant $J_{01}$ has been defined in Eq. (392).

\section{B.11 $\pi A$ VERTICES}

The interaction terms read

$$
\begin{aligned}
& H_{\pi A}^{(2)}=f_{\pi} \int \mathrm{d} \mathbf{x}\left(\mathbf{A}^{i} \cdot \partial_{i} \boldsymbol{\pi}+\boldsymbol{A}^{0} \cdot \boldsymbol{\Pi}\right), \\
& H_{\pi A}^{(4)}=\frac{2 m_{\pi}^{2} l_{4}}{f_{\pi}} \int \mathrm{d} \mathbf{x} \mathbf{A}^{i} \cdot \partial_{i} \boldsymbol{\pi},
\end{aligned}
$$

from which the vertices are obtained as

$$
\begin{aligned}
& \left\langle 0\left|H_{\pi A}^{(2)}\right| \mathbf{k}, a\right\rangle=i f_{\pi}\left(\mathbf{k} \cdot \mathbf{A}_{a}-\omega A_{a}^{0}\right) \\
& \left\langle 0\left|H_{\pi A}^{(4)}\right| \mathbf{k}, a\right\rangle=2 i \frac{m_{\pi}^{2} l_{4}}{f_{\pi}} \mathbf{k} \cdot \mathbf{A}_{a} .
\end{aligned}
$$

\section{B.12 $3 \pi A$ VERTEX}

The interaction term reads

$$
\begin{aligned}
H_{3 \pi A}^{(2)}= & \frac{1}{2 f_{\pi}} \int \mathrm{d} \mathbf{x}\left[2(1-2 \alpha) \mathbf{A}^{i} \cdot \boldsymbol{\pi} \boldsymbol{\pi} \cdot \partial_{i} \boldsymbol{\pi}-(2 \alpha+1) \mathbf{A}^{i} \cdot \partial_{i} \boldsymbol{\pi} \boldsymbol{\pi} \cdot \boldsymbol{\pi}\right. \\
& \left.+2(\alpha-1 / 2) A_{a}^{0} \pi_{b} \Pi_{a} \pi_{b}+2 \alpha A_{a}^{0}\left(\pi_{a} \boldsymbol{\pi} \cdot \boldsymbol{\Pi}+\boldsymbol{\Pi} \cdot \boldsymbol{\pi} \pi_{a}\right)\right],
\end{aligned}
$$


which leads to the following vertex

$$
\begin{aligned}
\left\langle 0\left|H_{3 \pi A}^{(2)}\right| \mathbf{k}_{1}, a_{1} ; \mathbf{k}_{2}, a_{2} ; \mathbf{k}_{3}, a_{3}\right\rangle=\frac{i}{f_{\pi}} & {\left[\delta_{a_{2}, a_{3}} \mathbf{A}_{a_{1}} \cdot\left[(2 \alpha-1) \mathbf{q}-2 \mathbf{k}_{1}\right]\right.} \\
& +\delta_{a_{1}, a_{3}} \mathbf{A}_{a_{2}} \cdot\left[(2 \alpha-1) \mathbf{q}-2 \mathbf{k}_{2}\right] \\
& +\delta_{a_{1}, a_{2}} \mathbf{A}_{a_{3}} \cdot\left[(2 \alpha-1) \mathbf{q}-2 \mathbf{k}_{3}\right] \\
& -\delta_{a_{2}, a_{3}} A_{a_{1}}^{0}\left[2 \alpha\left(\omega_{1}+\omega_{2}+\omega_{3}\right)-\omega_{1}\right] \\
& -\delta_{a_{1}, a_{3}} A_{a_{2}}^{0}\left[2 \alpha\left(\omega_{1}+\omega_{2}+\omega_{3}\right)-\omega_{2}\right] \\
& \left.-\delta_{a_{1}, a_{2}} A_{a_{3}}^{0}\left[2 \alpha\left(\omega_{1}+\omega_{2}+\omega_{3}\right)-\omega_{3}\right]\right],
\end{aligned}
$$

where in the first three lines use has been made of the $\delta$-function $(2 \pi)^{3} \delta\left(\mathbf{k}_{1}+\mathbf{k}_{2}+\mathbf{k}_{3}+\mathbf{q}\right)$. The tadpole contribution is found to be

$$
\left\langle 0\left|H_{3 \pi A}^{(2)}\right| \mathbf{k}, a\right\rangle=-\frac{i}{2 f_{\pi}} J_{01}\left[(5 \alpha+1 / 2) \mathbf{A}_{a} \cdot \mathbf{k}+(5 \alpha-3 / 2) A_{a}^{0} \omega\right] .
$$




\section{APPENDIX C}

\section{COUNTER-TERMS TO ORDER $Q^{3}$}

In this section we construct the counter-terms corresponding to the Lagrangian in Eq. (68). The expressions below are obtained in the presence of the external axial field. The counter-terms needed for the renormalization of the OPE potential discussed in Sec. 4.1.2 follow easily from these by ignoring the terms proportional to $\mathbf{A}^{\mu}$.

Having made the replacements in Eqs. (124)-(128), the bare Lagrangian $\mathcal{L}$ of Eq. (68) can be rewritten in terms of the renormalized fields and physical masses as

$$
\mathcal{L}=\mathcal{L}^{r}+\delta \mathcal{L}^{r},
$$

where $\mathcal{L}^{r}$ is the same as in Eq. (68) but now in terms of renormalized fields and masses, and $\delta \mathcal{L}^{r}$ includes the set of counter-terms

$$
\begin{aligned}
\delta \mathcal{L}^{r}= & \delta m \bar{N}^{r} N^{r}+\delta Z_{N} \bar{N}^{r}\left(i \gamma^{\mu} \partial_{\mu}-m^{r}\right) N^{r}+\delta Z_{N} \bar{N}^{r}\left[\Gamma_{a}^{0, r}(0) \partial_{0} \pi_{a}^{r}+\Lambda_{a}^{i, r}(0) \partial_{i} \pi_{a}^{r}+\Delta^{r}(1)\right] N^{r} \\
& +\delta Z_{\pi} \bar{N}^{r}\left[\left[\Gamma^{0, r}(0)+\delta \Gamma_{a}^{0, r}(0)\right] \partial_{0} \pi_{a}^{r}+\left[\Lambda_{a}^{i, r}(0) / 2+\delta \Lambda_{a}^{i, r}(0)\right] \partial_{i} \pi_{a}^{r}+\delta \Delta^{r}(1)\right] N^{r} \\
& +\frac{\delta m_{\pi}^{2}}{2} \pi_{a}^{r} \pi_{a}^{r}+\frac{\delta Z_{\pi}}{2}\left[\partial_{0} \pi_{a}^{r}\left(\widetilde{G}_{a b}^{r}+\delta \widetilde{G}_{a b}^{r}\right) \partial^{0} \pi_{b}^{r}+\partial_{i} \pi_{a}^{r}\left(\widetilde{G}_{a b}^{r}+\delta \widetilde{G}_{a b}^{r}\right) \partial^{i} \pi_{b}^{r}\right. \\
& \left.-m_{\pi}^{r 2} \pi_{a}^{r}\left(H_{a b}^{r}+\delta H_{a b}^{r}\right) \pi_{b}^{r}\right]-\delta Z_{\pi} f_{\pi} A_{a}^{\mu}\left(F_{a b}^{r} / 2+\delta F_{a b}^{r}\right) \partial_{\mu} \pi_{b}^{r}
\end{aligned}
$$

where $\Gamma_{a}^{0, r}(0), \Lambda_{a}^{i, r}(0)$ and $\Delta^{r}(1)$ are the field combinations defined in Eqs. (379), (380) and (381) expressed in terms of renormalized fields and physical masses. The remaining 
quantities are given by

$$
\begin{aligned}
\delta \Gamma_{a}^{0 r}(0)= & \frac{8 \alpha-1}{8 f_{\pi}^{4}} \boldsymbol{\pi}^{r} \cdot \boldsymbol{\pi}^{r}\left(\boldsymbol{\tau} \times \boldsymbol{\pi}^{r}\right)_{a} \gamma^{0}, \\
\delta \Lambda_{a}^{i, r}(0)= & \frac{g_{A}}{4 f_{\pi}^{3}}\left[2 \alpha \boldsymbol{\pi}^{r} \cdot \boldsymbol{\pi}^{r} \tau_{a}+(4 \alpha-1) \boldsymbol{\tau} \cdot \boldsymbol{\pi}^{r} \pi_{a}^{r}\right] \gamma^{i} \gamma_{5}, \\
\delta \Delta^{r}(1)= & \frac{1}{4 f_{\pi}}\left(1-\frac{3 \alpha}{f_{\pi}^{2}} \boldsymbol{\pi}^{r} \cdot \boldsymbol{\pi}^{r}\right)\left(\boldsymbol{\tau} \times \boldsymbol{\pi}^{r}\right) \cdot \mathbf{A}_{0} \gamma^{0} \\
& +\frac{g_{A}}{4 f_{\pi}^{2}}\left[\left(\boldsymbol{\tau} \times \boldsymbol{\pi}^{r}\right) \times \boldsymbol{\pi}^{r}\right] \cdot \mathbf{A}_{i} \gamma^{i} \gamma_{5}, \\
\delta \widetilde{G}_{a b}^{r}= & -\frac{2 \alpha}{f_{\pi}^{2}} \boldsymbol{\pi}^{r} \cdot \boldsymbol{\pi}^{r} \delta_{a b}+\frac{1-4 \alpha}{f_{\pi}^{2}} \pi_{a}^{r} \pi_{b}^{r}, \\
\delta H_{a b}^{r}= & \frac{1-8 \alpha}{4 f_{\pi}^{2}} \boldsymbol{\pi}^{r} \cdot \boldsymbol{\pi}^{r} \delta_{a b}, \\
\delta F_{a b}^{r}= & -\frac{2 \alpha+1}{2 f_{\pi}^{2}} \boldsymbol{\pi}^{r} \cdot \boldsymbol{\pi}^{r} \delta_{a b}+\frac{1-2 \alpha}{f_{\pi}^{2}} \pi_{a}^{r} \pi_{b}^{r} .
\end{aligned}
$$

It is convenient to define

$$
\begin{aligned}
\widetilde{G}_{a b}^{\prime} & =\widetilde{G}_{a b}^{r}+\delta Z_{\pi}\left(\widetilde{G}_{a b}^{r}+\delta \widetilde{G}_{a b}^{r}\right), \\
G_{a b}^{\prime} & =\widetilde{G}_{a b}^{\prime}+2 \frac{c_{2}+c_{3}}{f_{\pi}^{2}} \bar{N}^{r} N^{r} \delta_{a b}, \\
F_{a b}^{\prime} & =F_{a b}^{r}+\delta Z_{\pi}\left(F_{a b}^{r} / 2+\delta F_{a b}^{r}\right), \\
H_{a b}^{\prime} & =H_{a b}^{r}+\delta Z_{\pi}\left(H_{a b}^{r}+\delta H_{a b}^{r}\right), \\
\Gamma_{a}^{0 \prime} & =\Gamma_{a}^{0, r}+\delta Z_{N} \Gamma_{a}^{0, r}(0)+\delta Z_{\pi}\left[\Gamma_{a}^{0, r}(0)+\delta \Gamma_{a}^{0, r}(0)\right], \\
\Lambda_{a}^{i \prime} & =\Lambda_{a}^{i, r}+\delta Z_{N} \Lambda_{a}^{i, r}(0)+\delta Z_{\pi}\left[\Lambda_{a}^{i, r}(0) / 2+\delta \Lambda_{a}^{i, r}(0)\right], \\
\Delta^{\prime} & =\Delta^{r}+\delta Z_{N} \Delta^{r}(1)+\delta Z_{\pi} \delta \Delta^{r}(1),
\end{aligned}
$$

which then leads to the Lagrangian as given in Eq. (202). The interaction Hamiltonian in terms of the renormalized fields and physical masses is given in Eq. (203). 


\section{APPENDIX D}

\section{FIELD AND MASS RENORMALIZATION OF THE NUCLEON AND PION}

In this appendix we discuss mass and wave function renormalization of the nucleon and pion in TOPT. We closely follow the treatment Ref. [47].

We begin by discussing the nucleon case. The relevant interaction Hamiltonians are given by Eq. (203) of the previous Appendix, and read

$$
\begin{aligned}
H_{N N}^{(2)} & =\int \mathrm{d} \mathbf{x}\left(\delta M \bar{N} N-4 m_{\pi}^{2} c_{1} \bar{N} N\right), \\
H_{N N}^{(4)} & =-\delta Z_{N} \int \mathrm{d} \mathbf{x} \bar{N}\left(i \gamma^{i} \partial_{i}-M_{N}\right) N, \\
H_{\pi N N}^{(1)} & =\frac{g_{A}}{2 f_{\pi}} \int \mathrm{d} \mathbf{x} \bar{N} \boldsymbol{\tau} \cdot \partial_{i} \boldsymbol{\pi} \gamma^{i} \gamma^{5} N .
\end{aligned}
$$

The transition amplitude for a single nucleon is given by the diagrams in Fig. 31.

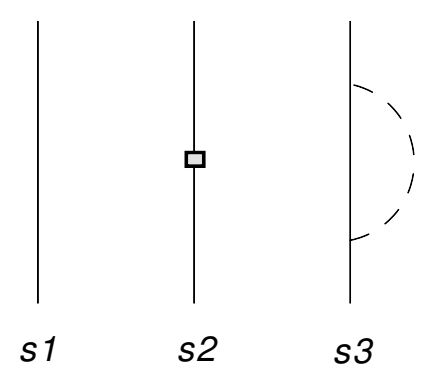

FIG. 31: Diagrams relevant for $\delta M$ and $Z_{N}$.

The contributions s2 and s3 are

$$
\begin{aligned}
\mathrm{s} 2 & =\delta M-4 m_{\pi}^{2} c_{1}+\delta Z_{N} E_{p} \\
\mathrm{~s} 3 & =\frac{3 g_{A}^{2}}{8 f_{\pi}^{2}} \int \frac{d \mathbf{k}}{(2 \pi)^{3}} \frac{\mathbf{k}^{2}}{\omega_{k}} \frac{1}{E_{p}-\left(E_{p-k}+\omega_{k}\right)+i \epsilon} .
\end{aligned}
$$

Diagram s3 at order $Q^{3}$ gives

$$
\mathrm{s} 3=-\frac{3 g_{A}^{2}}{8 f_{\pi}^{2}} J_{12}
$$


where $J_{12}$ has been defined in Eq. (392). We set the $N \rightarrow N$ amplitude to zero order by order in the power counting, assuming

$$
\delta M=\delta M^{(2)}+\delta M^{(3)}+\ldots,
$$

where $\delta M^{(n)}$ is of order $Q^{n}$. Therefore we obtain

$$
\delta M^{(2)}=4 m_{\pi}^{2} c_{1}, \quad \delta M^{(3)}=\frac{3 g_{A}^{2}}{8 f_{\pi}^{2}} J_{12},
$$

and the correction $\delta Z_{N} E_{p}$ (of order $Q^{4}$ ) is fixed below.

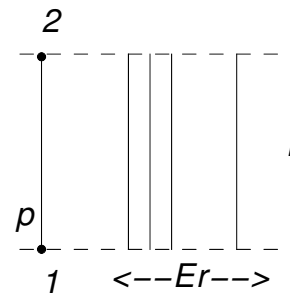

a

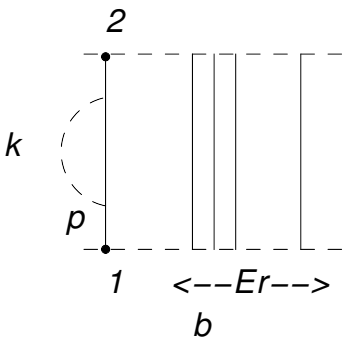

$b$

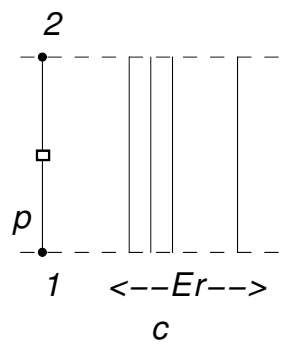

C

FIG. 32: Parts of a general diagram with the propagation of nucleons only.

Next we consider the "dressing" of a nucleon line belonging to a more complicated diagram, see Fig. 32. Panel a on this figure represents a diagram in which one nucleon of momentum $\mathbf{p}$ is created at vertex 1 and annihilated at vertex 2 (shown by the two dots at the beginning and end of the nucleon line). The other nucleons have energies collectively denoted by $E_{r}$. Note that there are no pions in flight in the intermediate state. The energy denominator of diagram a is

$$
P_{0}(E)=\frac{1}{E_{i}-\left(E_{p}+E_{r}\right)+i \epsilon}=\frac{1}{E+i \epsilon},
$$

where $E=E_{i}-E_{p}-E_{r}$ and $E_{i}$ is the initial energy (which depends on the particular process under consideration).

Diagrams b and c in Fig. 32 represent, respectively, the contribution in which nucleon 1 emits and reabsorbs a pion of momentum $\mathbf{k}$ and that in which a contact interaction occurs. These contributions are given by

$$
S(E)=\int \frac{d \mathbf{k}}{(2 \pi)^{3}} \frac{3 g_{A}^{2}}{8 f_{\pi}^{2}} \frac{k^{2}}{\omega_{k}} \frac{1}{E+E_{p}-E_{|\mathbf{p}-\mathbf{k}|}-\omega_{k}}=-4 m_{\pi}^{2} c_{1}+\delta M+\ldots,
$$

and $S(0)=0$ follows from the choice of $\delta M$ discussed previously for a single nucleon (of course, energy denominators in the diagrams of Figs. 31 and 32 are different, and $S(E)$ only vanishes for $E=0)$. 
By summing up repeated b- and c-type insertions, we obtain the well known result

$$
\begin{aligned}
P_{D}(E) & =\frac{1}{E+i \epsilon}+\frac{1}{E+i \epsilon} S(E) \frac{1}{E+i \epsilon}+\cdots \\
& =\frac{1}{E-S(E)+i \epsilon} .
\end{aligned}
$$

By expanding $S(E)$ in powers of $E$ ( $E$ is assumed to be small) and by keeping only linear terms in $E$, we find

$$
P_{D}(E) \simeq \frac{1}{1-S^{\prime}(0)} \frac{1}{E+i \epsilon}=\frac{Z_{N}}{E+i \epsilon}
$$

where $Z_{N}=1 /\left[1-S^{\prime}(0)\right]$,

$$
S^{\prime}(0)=-\int \frac{d \mathbf{k}}{(2 \pi)^{3}} \frac{3 g_{A}^{2}}{8 f_{\pi}^{2}} \frac{k^{2}}{\omega_{k}^{3}}=-\frac{3 g_{A}^{2}}{8 f_{\pi}^{2}} J_{13} .
$$

Since $-E=E_{p}+E_{r}-E_{i}$ is the energy of the intermediate state relative to the initial energy, it is physically sensible that for $E \rightarrow 0$ the dressed operator should have the same form as the bare propagator $1 /(E+i \epsilon)$ up to the (nucleon wave function) renormalization factor $Z_{N}$. In the following we adopt the common practice of attaching a $\sqrt{Z_{N}}$ at each of the two vertices of an internal nucleon line, and of multiplying by an extra $\sqrt{Z_{N}}$ each external nucleon line. The renormalization of nucleon lines when additional pions are present must be discussed case by case.

In the case of the pion the relevant interaction Hamiltonians from Eq. (203) are

$$
\begin{aligned}
H_{2 \pi}^{(4) \prime}= & \int \mathrm{d} \mathbf{x}\left[-\frac{m_{\pi}^{r 2} l_{4}}{f_{\pi}^{2}}\left(\boldsymbol{\Pi}^{r} \cdot \boldsymbol{\Pi}^{r}+\partial^{i} \boldsymbol{\pi}^{r} \cdot \partial_{i} \boldsymbol{\pi}^{r}\right)+\frac{m_{\pi}^{r 4}\left(l_{3}+l_{4}\right)}{f_{\pi}^{2}} \boldsymbol{\pi}^{r} \cdot \boldsymbol{\pi}^{r}\right. \\
& \left.-\frac{\delta Z_{\pi}}{2}\left(\boldsymbol{\Pi}^{r} \cdot \boldsymbol{\Pi}^{r}+\partial_{i} \boldsymbol{\pi}^{r} \cdot \partial^{i} \boldsymbol{\pi}^{r}-m_{\pi}^{r 2} \boldsymbol{\pi}^{r} \cdot \boldsymbol{\pi}^{r}\right)-\frac{\delta m^{2}}{2} \boldsymbol{\pi}^{r} \cdot \boldsymbol{\pi}^{r}\right], \\
H_{4 \pi}^{(2) \prime}= & \int \mathrm{d} \mathbf{x}\left[\frac{4 \alpha-1}{2 f_{\pi}^{2}}\left(\boldsymbol{\pi}^{r} \cdot \boldsymbol{\Pi}^{r} \boldsymbol{\Pi}^{r} \cdot \boldsymbol{\pi}^{r}+\partial_{i} \boldsymbol{\pi}^{r} \cdot \boldsymbol{\pi}^{r} \partial^{i} \boldsymbol{\pi}^{r} \cdot \boldsymbol{\pi}^{r}\right)\right. \\
& \left.+\frac{\alpha}{f_{\pi}^{2}}\left(\pi_{a}^{r} \boldsymbol{\Pi}^{r} \cdot \boldsymbol{\Pi}^{r} \pi_{a}^{r}+\boldsymbol{\pi}^{r 2} \partial_{i} \boldsymbol{\pi}^{r} \cdot \partial^{i} \boldsymbol{\pi}^{r}\right)-\frac{8 \alpha-1}{8 f_{\pi}^{2}} m_{\pi}^{r 2} \boldsymbol{\pi}^{r 4}\right] .
\end{aligned}
$$

with vertices (in the convention of Appendix B) given by

$$
\begin{aligned}
\left\langle 0\left|H_{2 \pi}^{(4) \prime}\right| \mathbf{k}_{1}, a_{1} ; \mathbf{k}_{2}, a_{2}\right\rangle= & \delta_{a_{1}, a_{2}}\left[\frac{2 m_{\pi}^{2} l_{4}}{f_{\pi}^{2}}\left(\omega_{1} \omega_{2}-\mathbf{k}_{1} \cdot \mathbf{k}_{2}\right)+\frac{2 m_{\pi}^{4}\left(l_{3}+l_{4}\right)}{f_{\pi}^{2}}\right. \\
& \left.+\delta Z_{\pi}\left(\omega_{1} \omega_{2}-\mathbf{k}_{1} \cdot \mathbf{k}_{2}+m_{\pi}^{2}\right)-\delta m^{2}\right], \\
\left\langle 0\left|H_{4 \pi}^{(2) \prime}\right| \mathbf{k}_{1}, a_{1} ; \mathbf{k}_{2}, a_{2}\right\rangle= & \delta_{a_{1}, a_{2}} J_{01}\left[\frac{1-10 \alpha}{2 f_{\pi}^{2}}\left(\omega_{1} \omega_{2}-\mathbf{k}_{1} \cdot \mathbf{k}_{2}\right)-\frac{20 \alpha-3}{4 f_{\pi}^{2}} m_{\pi}^{2}\right] .
\end{aligned}
$$


Considerations similar to those obtained above for the nucleon lead to

$$
\begin{aligned}
\delta m_{\pi}^{2} & =2 l_{3} \frac{m_{\pi}^{r 4}}{f_{\pi}^{2}}+\frac{m_{\pi}^{r 2}}{4 f_{\pi}^{2}} J_{01} \\
\delta Z_{\pi} & =-2 \frac{m_{\pi}^{r 2}}{f_{\pi}^{2}} l_{4}+\frac{10 \alpha-1}{2 f_{\pi}^{2}} J_{01} .
\end{aligned}
$$




\section{APPENDIX E}

\section{RENORMALIZATION OF THE RATIO $g_{A} / f_{\pi}$}

The relevant Hamiltonians are

$$
\begin{aligned}
H_{3 \pi N N}^{(1) \prime} & =-\frac{g_{A}}{2 f_{\pi}^{3}} \int \mathrm{d} \mathbf{x} \bar{N}^{r}\left[\alpha \pi^{r 2} \boldsymbol{\tau} \cdot \partial_{i} \boldsymbol{\pi}^{r}+\frac{1}{2}(4 \alpha-1) \boldsymbol{\tau} \cdot \boldsymbol{\pi}^{r} \boldsymbol{\pi}^{r} \cdot \partial_{i} \boldsymbol{\pi}^{r}\right] \gamma^{i} \gamma^{5} N^{r} \\
H_{\pi N N}^{(3) \prime} & =\int \mathrm{d} \mathbf{x}\left[\frac{m_{\pi}^{r 2}}{f_{\pi}}\left(2 d_{16}-d_{18}\right)+\frac{g_{A}}{2 f_{\pi}}\left(\delta Z_{N}+\frac{\delta Z_{\pi}}{2}\right)\right] \bar{N}^{r} \boldsymbol{\tau} \cdot \partial_{i} \boldsymbol{\pi}^{r} \gamma^{i} \gamma^{5} N^{r}
\end{aligned}
$$

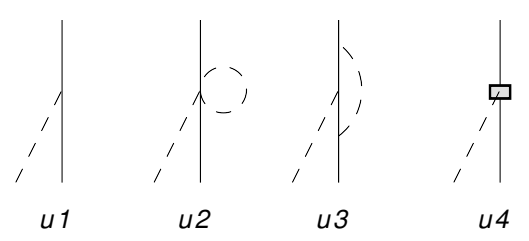

FIG. 33: Diagrams relevant for the renormalization of $g_{A} / f_{\pi}$.

The contributions of diagrams in Fig. 33 are

$$
\begin{aligned}
u 1 & =i \frac{g_{A}}{2 f_{\pi}} \boldsymbol{\sigma} \cdot \mathbf{k} \tau_{a}, \\
u 2 & =-i \frac{g_{A}}{8 f_{\pi}^{3}}(10 \alpha-1) J_{01} \boldsymbol{\sigma} \cdot \mathbf{k} \tau_{a}, \\
u 3 & =i \frac{g_{A}^{2}}{48 f_{\pi}^{3}} J_{13} \boldsymbol{\sigma} \cdot \mathbf{k} \tau_{a}, \\
u 4 & =i\left[\frac{m_{\pi}^{2}}{f_{\pi}}\left(2 d_{16}-d_{18}\right)-\frac{3 g_{A}^{3}}{16 f_{\pi}^{3}}+\frac{g_{A}}{4 f_{\pi}^{3}}\left(-2 m_{\pi}^{2} l_{4}+\frac{10 \alpha-1}{2}\right) J_{01}\right] \boldsymbol{\sigma} \cdot \mathbf{k} \tau_{a},
\end{aligned}
$$

and their sum reads

$$
u 1+u 2+u 3+u 4=i \frac{g_{A}}{2 f_{\pi}} \boldsymbol{\sigma} \cdot \mathbf{k} \tau_{a}\left[1+\frac{2 m_{\pi}^{2}}{g_{A}}\left(2 d_{16}-d_{18}\right)-\frac{g_{A}^{2}}{3 f_{\pi}^{2}} J_{13}-\frac{m_{\pi}^{2} l_{4}}{f_{\pi}^{2}}\right] .
$$

In terms of the renormalized coupling, we must have

$$
i \frac{g_{A}^{r}}{2 f_{\pi}^{r}} \boldsymbol{\sigma} \cdot \mathbf{k} \tau_{a}=u 1+u 2+u 3+u 4
$$


which implies (to order $Q^{2}$ )

$$
\begin{aligned}
\frac{g_{A}^{r}}{f_{\pi}^{r}} & =\frac{g_{A}}{f_{\pi}}\left[1+\frac{m_{\pi}^{2}}{g_{A}}\left(4 d_{16}-2 d_{18}\right)-\frac{g_{A}^{2}}{3 f_{\pi}^{2}} J_{13}-\frac{m_{\pi}^{2} l_{4}}{f_{\pi}^{2}}\right] \\
& =\frac{g_{A}}{f_{\pi}}\left(1+\frac{4 m_{\pi}^{2}}{g_{A}} d_{16}-\frac{g_{A}^{2}}{3 f_{\pi}^{2}} J_{13}-\frac{m_{\pi}^{2} l_{4}}{f_{\pi}^{2}}\right)\left(1-\frac{2 m_{\pi}^{2}}{g_{A}} d_{18}\right)
\end{aligned}
$$

where in the second line we have isolated the Goldberger-Treiman discrepancy [47]. The quantity $J_{13}$ is ultraviolet divergent, and in dimensional regularization is given by

$$
J_{13}=\frac{3 m_{\pi}^{2}}{8 \pi^{2}}\left(d_{\epsilon}-\frac{7}{3}\right)
$$

where $d_{\epsilon}$ has been defined in Eq. (110). The divergent parts of $l_{i}$ 's and $d_{i}$ 's have been identified in the heavy-baryon formalism, without considering any specific process, with the background-field and heat-kernel methods (se Ref. [53], and references therein). We report below the expressions for these divergent parts from Table 4 of that work:

$$
\begin{aligned}
l_{i} & =\gamma_{i} \lambda+l_{i}^{r}(\mu), \\
d_{i} & =\frac{\beta_{i}}{f_{\pi}^{2}} \lambda+d_{i}^{r}(\mu),
\end{aligned}
$$

where, in the conventions adopted in the present work,

$$
\begin{aligned}
\lambda & =\frac{1}{32 \pi^{2}}\left(d_{\epsilon}+\ln \frac{\mu^{2}}{m_{\pi}^{2}}\right), \\
l_{i}^{r}(\mu) & =\frac{\gamma_{i}}{32 \pi^{2} f_{\pi}^{2}} \ln \frac{m_{\pi}^{2}}{\mu^{2}}+l_{i}^{r}\left(m_{\pi}\right), \\
d_{i}^{r}(\mu) & =\frac{\beta_{i}}{32 \pi^{2} f_{\pi}^{2}} \ln \frac{m_{\pi}^{2}}{\mu^{2}}+d_{i}^{r}\left(m_{\pi}\right),
\end{aligned}
$$

where $l_{i}^{r}\left(m_{\pi}\right)$ and $d_{i}^{r}\left(m_{\pi}\right)$ are the physical (i.e., scale independent) LECs from $\mathcal{L}_{\pi \pi}^{(4)}$ and $\mathcal{L}_{\pi N}^{(3)}$. The $\gamma_{i}$ and $\beta_{i}$ functions of interest here are those relative to $l_{4}$ and $d_{16}$ respectively

$$
\begin{aligned}
\gamma_{4} & =2, \\
\beta_{16} & =\frac{1}{2} g_{A}+g_{A}^{3} .
\end{aligned}
$$

We note that LEC $d_{18}$ has no divergent part. As we can see divergencies, cancel out exactly in Eq. (481), and we are left with

$$
\frac{g_{A}^{r}}{f_{\pi}^{r}}=\frac{g_{A}}{f_{\pi}}\left(1+\frac{4 m_{\pi}^{r 2}}{g_{A}^{r}} d_{16}^{r}-\frac{7 g_{A}^{r 2}}{12 \pi^{2} f_{\pi}^{r 2}} m_{\pi}^{r 2}-\frac{m_{\pi}^{r 2} l_{4}^{r}}{f_{\pi}^{r 2}}\right),
$$

where on the r.h.s. we have replaced $g_{A}, f_{\pi}, m_{\pi}$ by their renormalized quantities, which is correct to the order $Q^{2}$ we are considering here. Note that

$$
\frac{g_{A}}{f_{\pi}}=\frac{g_{A}^{r}}{f_{\pi}^{r}}\left(1-\frac{4 m_{\pi}^{r 2}}{g_{A}^{r}} d_{16}^{r}+\frac{7 g_{A}^{r 2}}{12 \pi^{2} f_{\pi}^{r 2}} m_{\pi}^{r 2}+\frac{m_{\pi}^{r 2} l_{4}^{r}}{f_{\pi}^{r 2}}\right) .
$$




\section{APPENDIX F}

\section{RENORMALIZATION OF THE ONE-BODY AXIAL}

\section{CURRENT}

We first discuss the renormalzation of the pion decay constant $f_{\pi}$. The relevant interaction Hamiltonians are

$$
\begin{aligned}
H_{\pi A}^{(2) \prime}= & f_{\pi} \int \mathrm{d} \mathbf{x}\left(\mathbf{A}^{i} \cdot \partial_{i} \boldsymbol{\pi}^{r}+\mathbf{A}^{0} \cdot \boldsymbol{\Pi}^{r}\right) \\
H_{3 \pi A}^{(2) \prime}= & \frac{1}{2 f_{\pi}} \int \mathrm{d} \mathbf{x}\left[2(1-2 \alpha) \mathbf{A}^{i} \cdot \boldsymbol{\pi}^{r} \boldsymbol{\pi}^{r} \cdot \partial_{i} \boldsymbol{\pi}^{r}-(2 \alpha+1) \mathbf{A}^{i} \cdot \partial_{i} \boldsymbol{\pi}^{r} \boldsymbol{\pi}^{r} \cdot \boldsymbol{\pi}^{r}\right. \\
& \left.+2(\alpha-1 / 2) A_{a}^{0} \pi_{b}^{r} \Pi_{a}^{r} \pi_{b}^{r}+2 \alpha A_{a}^{0}\left(\pi_{a}^{r} \boldsymbol{\pi}^{r} \cdot \boldsymbol{\Pi}^{r}+\boldsymbol{\Pi}^{r} \cdot \boldsymbol{\pi}^{r} \pi_{a}^{r}\right)\right] \\
H_{\pi A}^{(4) \prime}= & \int \mathrm{d} \mathbf{x}\left[\frac{2 m_{\pi}^{r 2} l_{4}}{f_{\pi}} \mathbf{A}^{i} \cdot \partial_{i} \boldsymbol{\pi}^{r}-\frac{\delta Z_{\pi}}{2} f_{\pi}\left(-\mathbf{A}^{i} \cdot \partial_{i} \boldsymbol{\pi}^{r}+\mathbf{A}^{0} \cdot \boldsymbol{\Pi}^{r}\right)\right]
\end{aligned}
$$

where $H_{\pi A}^{(2) \prime}$ and $H_{3 \pi A}^{(2) \prime}$ are the same as in Eqs. (432) and (436) but in terms of renormalized pion field and mass, while $H_{\pi A}^{(4) \prime}$ relative to Eq. (433) includes counter-terms. The contributions illustrated in Fig. 34 read

$$
\begin{aligned}
& \mathrm{a} 1=-i f_{\pi}\left(\mathbf{k} \cdot \mathbf{A}_{a}-\omega A_{a}^{0}\right) \\
& \mathrm{a} 2=-\frac{i}{2 f_{\pi}} J_{01}\left[-(5 \alpha+1 / 2) \mathbf{A}_{a} \cdot \mathbf{k}-(5 \alpha-3 / 2) A_{a}^{0} \omega\right] \text {, } \\
& \mathrm{a} 3=-2 i \frac{m_{\pi}^{r 2} l_{4}}{f_{\pi}} \mathbf{k} \cdot \mathbf{A}_{a}+i \frac{\delta Z_{\pi}}{2} f_{\pi}\left(-\mathbf{k} \cdot \mathbf{A}_{a}-\omega A_{a}^{0}\right) . \\
& \sum_{a 1}^{1} \sum_{a 2}^{1} \sum_{a 3}^{1}
\end{aligned}
$$

FIG. 34: Diagrams relevant for the renormalization of $f_{\pi}$. 
We now require that the renormalized (physical) pion decay constant is equal to

$$
-i f_{\pi}^{r}\left(\mathbf{k} \cdot \mathbf{A}-\omega A_{a}^{0}\right)=\mathrm{a} 1+\mathrm{a} 2+\mathrm{a} 3
$$

implying

$$
f_{\pi}^{r}=f_{\pi}\left(1+\frac{m_{\pi}^{r 2} l_{4}}{f_{\pi}^{2}}-\frac{J_{01}}{2 f_{\pi}^{2}}\right)
$$

which to the order $Q^{2}$ of interest also gives

$$
f_{\pi}=f_{\pi}^{r}\left(1-\frac{m_{\pi}^{r 2} l_{4}}{f_{\pi}^{r 2}}+\frac{J_{01}}{2 f_{\pi}^{r 2}}\right) .
$$

This result is in accord with that obtained in Ref. [35].

We can now move on to discuss the renormalization of the axial coupling contant $g_{A}$ by considering the coupling to $\mathbf{A}_{a}$. The relevant interaction Hamiltonians are $H_{A N N}^{(1) \prime}$ and $H_{2 \pi N N A}^{(1) \prime}$ in Eqs. (410) and (420), and

$$
H_{A N N}^{(3) \prime}=-\int \mathrm{d} \mathbf{x} \bar{N}^{r}\left(2 m_{\pi}^{r 2} d_{16} \boldsymbol{\tau} \cdot \mathbf{A}_{i} \gamma^{i} \gamma_{5}+\delta Z_{N} \frac{g_{A}}{2} \boldsymbol{\tau} \cdot \mathbf{A}_{i} \gamma^{i} \gamma_{5}+\frac{d_{22}}{2} \boldsymbol{\tau} \cdot \partial^{j} \mathbf{F}_{i j} \gamma^{i} \gamma_{5}\right) N^{r}
$$

We consider a similar set of diagrams as in Fig. 34, but for the incoming pion line replaced by the external field. Their contributions are given by

$$
\begin{aligned}
\mathrm{b} 1 & =\frac{g_{A}}{2} \tau_{a} \boldsymbol{\sigma} \cdot \mathbf{A}_{a} \\
\mathrm{~b} 2 & =-\frac{g_{A}}{4 f_{\pi}^{2}} J_{01} \tau_{a} \boldsymbol{\sigma} \cdot \mathbf{A}_{a} \\
\mathrm{~b} 3 & =\frac{g_{A}^{3}}{48 f_{\pi}^{2}} J_{13} \tau_{a} \boldsymbol{\sigma} \cdot \mathbf{A}_{a} \\
\mathrm{~b} 4 & =\left(\frac{g_{A}}{2} \delta Z_{N}+2 m_{\pi}^{r 2} d_{16}\right) \tau_{a} \boldsymbol{\sigma} \cdot \mathbf{A}_{a}+\frac{d_{22}}{2} \tau_{a}[\mathbf{q} \times(\mathbf{q} \times \boldsymbol{\sigma})] \cdot \mathbf{A}_{a}
\end{aligned}
$$

and sum up to $\bar{g}_{A}^{r} \boldsymbol{\sigma} \tau_{a} / 2$, with the renormalized axial coupling constant (to order $Q^{2}$ ) obtained as

$$
\bar{g}_{A}^{r}=g_{A}\left[1-\frac{1}{2 f_{\pi}^{r 2}} J_{01}-\frac{g_{A}^{r^{2}}}{3 f_{\pi}^{r 2}} J_{13}+\frac{4 m_{\pi}^{r 2}}{g_{A}^{r}} d_{16}\right],
$$

and $\bar{g}_{A}^{r}$, apart from the Goldberger-Treiman discrepancy, is in agreement with Eq. (490). It is also in agreement with the results, to order $Q^{2}$, reported by Schindler et al. in Ref. [50]. The term proportional to $d_{22}$ quadratic in $\mathbf{q}$ contributes to the nucleon axial radius [50]. 


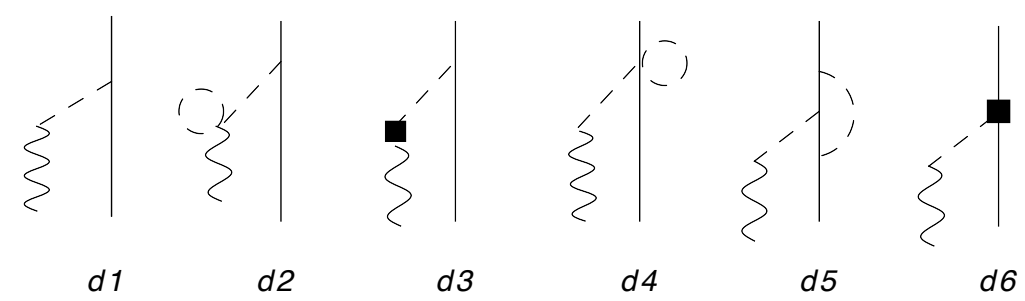

FIG. 35: Pion-pole diagrams.

Finally we examine the pion-pole contributions illustrated in Fig. 35. We obtain

$$
\begin{aligned}
\mathrm{d} 1= & -\frac{g_{A}}{2} \mathbf{A}_{a} \cdot \mathbf{q} \frac{\mathbf{q} \cdot \boldsymbol{\sigma}}{q^{2}+m_{\pi}^{r 2}} \tau_{a} \\
\mathrm{~d} 2+\mathrm{d} 3= & \frac{g_{A}}{2 f_{\pi}^{2}}\left(-m_{\pi}^{r 2} l_{4}+\frac{J_{01}}{2}\right) \mathbf{A}_{a} \cdot \mathbf{q} \frac{\mathbf{q} \cdot \boldsymbol{\sigma}}{q^{2}+m_{\pi}^{r 2}} \tau_{a} \\
\mathrm{~d} 4= & \frac{g_{A}}{8 f_{\pi}^{2}}(10 \alpha-1) J_{01} \mathbf{A}_{a} \cdot \mathbf{q} \frac{\mathbf{q} \cdot \boldsymbol{\sigma}}{q^{2}+m_{\pi}^{r 2}} \tau_{a} \\
\mathrm{~d} 5= & -\frac{g_{A}^{3}}{48 f_{\pi}^{2}} J_{13} \mathbf{A}_{a} \cdot \mathbf{q} \frac{\mathbf{q} \cdot \boldsymbol{\sigma}}{q^{2}+m_{\pi}^{r 2}} \tau_{a}, \\
\mathrm{~d} 6= & {\left[-m_{\pi}^{r 2}\left(2 d_{16}-d_{18}\right)+\frac{3 g_{A}^{3}}{16 f_{\pi}^{2}} J_{13}\right.} \\
& \left.-\frac{g_{A}}{4 f_{\pi}^{2}}\left(-2 m_{\pi}^{r 2} l_{4}+\frac{10 \alpha-1}{2} J_{01}\right)\right] \mathbf{A}_{a} \cdot \mathbf{q} \frac{\mathbf{q} \cdot \boldsymbol{\sigma}}{q^{2}+m_{\pi}^{r 2}} \tau_{a} .
\end{aligned}
$$

Their sum reads

$$
\begin{aligned}
\mathrm{d} 1+\cdots+\mathrm{d} 6= & -\frac{g_{A}}{2}\left[1-\frac{1}{2 f_{\pi}^{r 2}} J_{01}-\frac{g_{A}^{r 2}}{3 f_{\pi}^{r 2}} J_{13}+\frac{4 m_{\pi}^{r 2}}{g_{A}^{r}} d_{16}\right]\left(1-\frac{2 m_{\pi}^{r 2}}{g_{A}^{r}} d_{18}\right) \\
& \times \mathbf{A}_{a} \cdot \mathbf{q} \frac{\mathbf{q} \cdot \boldsymbol{\sigma}}{q^{2}+m_{\pi}^{r 2}} \tau_{a}
\end{aligned}
$$

and therefore the renormalized $g_{A}^{r}$ follows exactly as in Eq. (490), including the GoldbergerTreiman discrepancy. The renormalized (single-nucleon) current is then given by

$$
\mathbf{j}_{5, a}=-\frac{\bar{g}_{A}^{r}}{2} \boldsymbol{\sigma} \tau_{a}+\frac{g_{A}^{r}}{2} \mathbf{q} \frac{\mathbf{q} \cdot \boldsymbol{\sigma}}{q^{2}+m_{\pi}^{r 2}} \tau_{a},
$$

and this current is conserved in the chiral limit $\left(m_{\pi} \rightarrow 0\right)$, since in that limit $g_{A}^{r}=\bar{g}_{A}^{r}$. 


\section{APPENDIX G}

\section{CONTACT TERMS FOR THE AXIAL CHARGE CURRENT}

The weak-interaction potential $v_{5}=A_{a}^{0} \rho_{5, a}-\mathbf{A}_{a} \cdot \mathbf{j}_{5, a}$ is parity $(\mathcal{P})$ and time-reversal $(\mathcal{T})$ invariant, which implies that $\rho_{5, a} \stackrel{\mathcal{P}}{\longrightarrow}-\rho_{5, a}$ and $\mathbf{j}_{5, a} \stackrel{\mathcal{P}}{\longrightarrow} \mathbf{j}_{5, a}$, and $\rho_{5, a} \stackrel{\mathcal{T}}{\longrightarrow}(-)^{a+1} \rho_{5, a}$ and $\mathbf{j}_{5, a} \stackrel{\mathcal{T}}{\longrightarrow}(-)^{a} \mathbf{j}_{5, a}$. At order $Q^{0}$ there is no momentum dependence, and consequently there are no contact terms which can be constructed for $\rho_{5, a}$, while two such terms occur for $\mathbf{j}_{5, a}$, of which only one is independent (Fierz identities, see below) and is given in Eq. (173). At order $Q$ the contact terms in $\rho_{5, a}$ and $\mathbf{j}_{5, a}$ must be linear in either $\mathbf{k}_{i}=\mathbf{p}_{i}^{\prime}-\mathbf{p}_{i}$ or $\mathbf{K}_{i}=\left(\mathbf{p}_{i}^{\prime}+\mathbf{p}_{i}\right) / 2$ with $i=1$ and 2 . None can be constructed for $\mathbf{j}_{5, a}$. A complete, but non minimal, set of hermitian operators for the axial charge $\rho_{5, a}$ is the following:

$$
\begin{aligned}
& \widetilde{O}_{1}=\left(\tau_{1, a}+\tau_{2, a}\right)\left(\boldsymbol{\sigma}_{1}+\boldsymbol{\sigma}_{2}\right) \cdot\left(\mathbf{K}_{1}+\mathbf{K}_{2}\right), \\
& \widetilde{O}_{2}=\left(\tau_{1, a}+\tau_{2, a}\right)\left(\boldsymbol{\sigma}_{1}-\boldsymbol{\sigma}_{2}\right) \cdot\left(\mathbf{K}_{1}-\mathbf{K}_{2}\right), \\
& \widetilde{O}_{3}=i\left(\tau_{1, a}+\tau_{2, a}\right)\left(\boldsymbol{\sigma}_{1} \times \boldsymbol{\sigma}_{2}\right) \cdot\left(\mathbf{k}_{1}-\mathbf{k}_{2}\right), \\
& \widetilde{O}_{4}=\left(\tau_{1, a}-\tau_{2, a}\right)\left(\boldsymbol{\sigma}_{1}-\boldsymbol{\sigma}_{2}\right) \cdot\left(\mathbf{K}_{1}+\mathbf{K}_{2}\right), \\
& \widetilde{O}_{5}=\left(\tau_{1, a}-\tau_{2, a}\right)\left(\boldsymbol{\sigma}_{1}+\boldsymbol{\sigma}_{2}\right) \cdot\left(\mathbf{K}_{1}-\mathbf{K}_{2}\right), \\
& \widetilde{O}_{6}=i\left(\tau_{1, a}-\tau_{2, a}\right)\left(\boldsymbol{\sigma}_{1} \times \boldsymbol{\sigma}_{2}\right) \cdot\left(\mathbf{k}_{1}+\mathbf{k}_{2}\right), \\
& \widetilde{O}_{7}=i\left(\boldsymbol{\tau}_{1} \times \boldsymbol{\tau}_{2}\right)_{a}\left(\boldsymbol{\sigma}_{1}-\boldsymbol{\sigma}_{2}\right) \cdot\left(\mathbf{k}_{1}+\mathbf{k}_{2}\right), \\
& \widetilde{O}_{8}=i\left(\boldsymbol{\tau}_{1} \times \boldsymbol{\tau}_{2}\right)_{a}\left(\boldsymbol{\sigma}_{1}+\boldsymbol{\sigma}_{2}\right) \cdot\left(\mathbf{k}_{1}-\mathbf{k}_{2}\right), \\
& \widetilde{O}_{9}=\left(\boldsymbol{\tau}_{1} \times \boldsymbol{\tau}_{2}\right)_{a}\left(\boldsymbol{\sigma}_{1} \times \boldsymbol{\sigma}_{2}\right) \cdot\left(\mathbf{K}_{1}+\mathbf{K}_{2}\right) .
\end{aligned}
$$

The antisymmetry of initial and final two-nucleon states requires

$$
\widetilde{O}_{i}=-P^{\tau} P^{\sigma} P^{\text {space }} \widetilde{O}_{i}
$$

where $P^{\text {space }}$ is the space exchange operator, and $P^{\sigma}$ and $P^{\tau}$ are the spin and isospin exchange operators with $P^{\sigma}=\left(1+\boldsymbol{\sigma}_{1} \cdot \boldsymbol{\sigma}_{2}\right) / 2$ and similarly for $P^{\tau}$. Exchange of the final momenta of the two nucleons $\mathbf{p}_{1}^{\prime} \rightleftharpoons \mathbf{p}_{2}^{\prime}$ leads to

$$
\begin{aligned}
& P^{\text {space }}\left(\mathbf{k}_{1}+\mathbf{k}_{2}\right)=\mathbf{k}_{1}+\mathbf{k}_{2}, \quad P^{\text {space }}\left(\mathbf{k}_{1}-\mathbf{k}_{2}\right)=2\left(\mathbf{K}_{2}-\mathbf{K}_{1}\right), \\
& P^{\text {space }}\left(\mathbf{K}_{1}+\mathbf{K}_{2}\right)=\mathbf{K}_{1}+\mathbf{K}_{2}, \quad P^{\text {space }}\left(\mathbf{K}_{1}-\mathbf{K}_{2}\right)=\left(\mathbf{k}_{2}-\mathbf{k}_{1}\right) / 2 \text {, }
\end{aligned}
$$


while spin exchange implies

$$
P^{\sigma}\left(\boldsymbol{\sigma}_{1}+\boldsymbol{\sigma}_{2}\right)=\boldsymbol{\sigma}_{1}+\boldsymbol{\sigma}_{2}, P^{\sigma}\left(\boldsymbol{\sigma}_{1}-\boldsymbol{\sigma}_{2}\right)=i\left(\boldsymbol{\sigma}_{1} \times \boldsymbol{\sigma}_{2}\right), P^{\sigma}\left(\boldsymbol{\sigma}_{1} \times \boldsymbol{\sigma}_{2}\right)=-i\left(\boldsymbol{\sigma}_{1}-\boldsymbol{\sigma}_{2}\right)
$$

and similar relations follow under isospin exchange. The following (Fierz) identities are obtained from Eq. (514):

$$
\widetilde{O}_{2}=\widetilde{O}_{3} / 2, \quad \widetilde{O}_{4}=\widetilde{O}_{9}, \quad \widetilde{O}_{5}=\widetilde{O}_{8} / 2, \quad \widetilde{O}_{6}=-\widetilde{O}_{7},
$$

while $\widetilde{O}_{1}$ is required to vanish. Hence only 4 of the above 9 operators are independent, and a convenient set is

$$
O_{1}=\left(\widetilde{O}_{7}-\widetilde{O}_{8}\right) / 2, O_{2}=\left(\widetilde{O}_{7}+\widetilde{O}_{8}\right) / 2, O_{3}=\left(\widetilde{O}_{6}-\widetilde{O}_{3}\right) / 2, O_{4}=\widetilde{O}_{4}
$$

We note that $O_{1}$ and $O_{3}$ have the same operator structures associated with the divergent parts of the loop diagrams. 


\section{APPENDIX H}

\section{NONSTATIC CORRECTIONS TO ONE-BODY PION-POLE}

\section{DIAGRAM}

The one-body axial current, coming from the pion-pole term (diagrams a and b of Fig. 36), before the expansion of the energy denominator, reads

$$
\begin{aligned}
& \mathbf{j}_{5, a}(\mathrm{a})=\frac{g_{A}}{4} \tau_{1, a} \frac{\mathbf{q}}{E_{i}-E_{1}-\sqrt{q^{2}+m_{\pi}^{2}}} \frac{\boldsymbol{\sigma}_{1} \cdot \mathbf{q}}{\sqrt{q^{2}+m_{\pi}^{2}}}, \\
& \mathbf{j}_{5, a}(\mathrm{~b})=\frac{g_{A}}{4} \tau_{1, a} \frac{\mathbf{q}}{E_{i}-\omega_{q}-E_{1}^{\prime}-\sqrt{q^{2}+m_{\pi}^{2}}} \frac{\boldsymbol{\sigma}_{1} \cdot \mathbf{q}}{\sqrt{q^{2}+m_{\pi}^{2}}},
\end{aligned}
$$

where $E_{i}=\omega_{q}+E_{1}$ is the initial energy carried, respectively, by the external axial field and the nucleon, $\sqrt{q^{2}+m_{\pi}^{2}}$ is the pion energy, $q$ is the three-momenta of the axial field, and a momentum conserving $\delta$-function is understood.

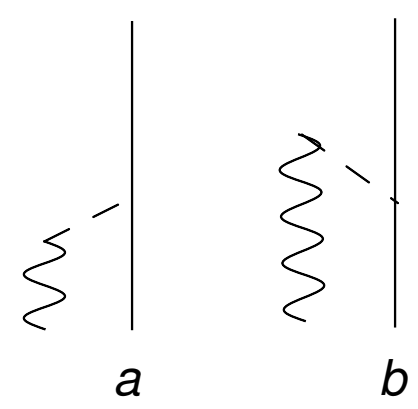

FIG. 36: Time orderings for the pion-pole one-body axial current topologies, indicated in diagram a 2 of Fig. 4. Nucleons, and pions are denoted by solid, and dashed lines, respectively. The second nucleon line is not displayed. See text for further explanations.

The LO contribution enters at order $Q^{-3}$, obtained by the LO expansion of energy denominators of diagrams $\mathrm{a}$ and $\mathrm{b}$ and it is the pion-pole analogue the one-body GT operator

$$
\mathbf{j}_{5, a}^{(-3)}(\mathrm{a}+\mathrm{b})=-\frac{g_{A}}{2} \tau_{1, a} \frac{\mathbf{q}}{q^{2}+m_{\pi}^{2}} \boldsymbol{\sigma}_{1} \cdot \mathbf{q} .
$$

The nonstatic correction at order $Q^{-2}$ turns out to vanish on the energy shell

$$
\mathbf{j}_{5, a}^{(-2)}(\mathrm{a}+\mathrm{b})=-\frac{g_{A}}{2} \tau_{1, a} \frac{\mathbf{q}}{\left(q^{2}+m_{\pi}^{2}\right)^{3 / 2}} \boldsymbol{\sigma}_{1} \cdot \mathbf{q}\left(2 E_{i}-E_{1}-E_{1}^{\prime}-\omega_{q}\right)=0 .
$$


We take it to vanish off-the-energy-shell too. At order $Q^{-1}$ Eq. (520) reads

$$
\begin{aligned}
\mathbf{j}_{5, a}^{(-1)}(\mathrm{a}+\mathrm{b}) & =-\frac{g_{A}}{2} \tau_{1, a} \frac{\mathbf{q}}{\left(q^{2}+m_{\pi}^{2}\right)^{2}} \boldsymbol{\sigma}_{1} \cdot \mathbf{q}\left(E_{1}^{\prime}-E_{1}\right)^{2} \\
& =-\frac{g_{A}}{2} \tau_{1, a} \frac{\mathbf{q}}{\left(q^{2}+m_{\pi}^{2}\right)^{2}} \boldsymbol{\sigma}_{1} \cdot \mathbf{q} \omega_{q}^{2}
\end{aligned}
$$

where the two forms are equivalent on the energy shell. In this Thesis the off-the-energyshell-extension of the one-body axial current at order $Q^{-1}$ used is the one reported in the second line of Eq. (524). 


\section{APPENDIX I}

\section{REGULARIZED LOOP CONTRIBUTIONS TO $\mathbf{j}_{5, a}^{\mathrm{MPE}}$}

The regularized contributions of diagrams in Fig. 10 read:

$$
\begin{aligned}
& \mathbf{j}_{5, a}^{(1)}(\mathrm{e} 1)=\frac{g_{A}^{3}}{64 \pi f_{\pi}^{4}} \tau_{2, a} \int_{0}^{1} d z\left[\boldsymbol{\sigma}_{1} M\left(k_{2}, z\right)+\mathbf{k}_{2} \boldsymbol{\sigma}_{1} \cdot \mathbf{k}_{2} \frac{z \bar{z}}{M\left(k_{2}, z\right)}\right], \\
& \mathbf{j}_{5, a}^{(1)}(\mathrm{e} 4)=-\frac{g_{A}^{3}}{64 \pi f_{\pi}^{4}} \tau_{2, a} \boldsymbol{\sigma}_{2} \int_{0}^{1} d z\left[\frac{k_{1}^{2} \bar{z} z}{M\left(k_{1}, z\right)}+3 M\left(k_{1}, z\right)\right], \\
& \mathbf{j}_{5, a}^{(1)}(\mathrm{e} 5)=\frac{g_{A}^{3}}{128 \pi f_{\pi}^{4}} \frac{\mathbf{q}}{q^{2}+m_{\pi}^{2}} \int_{0}^{1} d z\left[\tau_{2, a} \boldsymbol{\sigma}_{2} \cdot\left(\mathbf{k}_{1}-\mathbf{k}_{2}\right)\left[\frac{k_{1}^{2} z \bar{z}}{M\left(k_{1}, z\right)}+3 M\left(k_{1}, z\right)\right]\right. \\
& \left.-\left(\boldsymbol{\tau}_{1} \times \boldsymbol{\tau}_{2}\right)_{a}\left(\boldsymbol{\sigma}_{1} \times \boldsymbol{\sigma}_{2}\right) \cdot \mathbf{k}_{1} M\left(k_{1}, z\right)\right], \\
& \mathbf{j}_{5, a}^{(1)}(\mathrm{e} 8)=-\frac{g_{A}^{5}}{64 \pi f_{\pi}^{4}} \int_{0}^{1} d z\left[\tau _ { 2 , a } \left[5 \boldsymbol{\sigma}_{1} M\left(k_{2}, z\right)+\frac{\mathbf{k}_{2}}{2} \boldsymbol{\sigma}_{1} \cdot \mathbf{k}_{2}\left[\frac{k_{2}^{2}(z \bar{z})^{2}}{M\left(k_{2}, z\right)^{3}}+\frac{1-7 z \bar{z}}{M\left(k_{2}, z\right)}\right]\right.\right. \\
& \left.\left.+\frac{k_{2}^{2}}{2} \boldsymbol{\sigma}_{1}\left[\frac{9 z \bar{z}-1}{M\left(k_{2}, z\right)}-\frac{k_{2}^{2}(z \bar{z})^{2}}{M\left(k_{2}, z\right)^{3}}\right]\right]+\frac{\tau_{1, a}}{2}\left(\boldsymbol{\sigma}_{2} \times \mathbf{k}_{2}\right) \times \mathbf{k}_{2} \frac{1}{M\left(k_{2}, z\right)}\right], \\
& \mathbf{j}_{5, a}^{(1)}(\mathrm{e} 10)=\frac{g_{A}^{3}}{128 \pi f_{\pi}^{4}} \frac{\mathbf{q}}{q^{2}+m_{\pi}^{2}} \int_{0}^{1} d z\left[\left(2 \tau_{2, a}-\tau_{1, a}\right)\left[\frac{k_{2}^{2}}{M\left(k_{2}, z\right)}+3 M\left(k_{2}, z\right)\right] \boldsymbol{\sigma}_{1} \cdot \mathbf{k}_{2}\right. \\
& \left.+\left(\boldsymbol{\tau}_{1} \times \boldsymbol{\tau}_{2}\right)_{a} M\left(k_{2}, z\right)\left(\boldsymbol{\sigma}_{1} \times \boldsymbol{\sigma}_{2}\right) \cdot \mathbf{k}_{2}\right], \\
& \mathbf{j}_{5, a}^{(1)}(\mathrm{e} 15)=\frac{g_{A}^{3}}{128 \pi f_{\pi}^{4}} \int_{0}^{1} d z\left[\tau_{2, a}\left[\frac{k_{1}^{2} z \bar{z}}{M\left(k_{1}, z\right)}+3 M\left(k_{1}, z\right)\right]\left(\mathbf{k}_{2}-3 \mathbf{k}_{1}\right)\right. \\
& \left.+4\left(\boldsymbol{\tau}_{1} \times \boldsymbol{\tau}_{2}\right)_{a}\left(\boldsymbol{\sigma}_{1} \times \mathbf{k}_{1}\right) M\left(k_{1}, z\right)\right] \frac{\boldsymbol{\sigma}_{2} \cdot \mathbf{k}_{2}}{\omega_{2}^{2}}, \\
& \mathbf{j}_{5, a}^{(1)}(\mathrm{e} 16)=\frac{g_{A}^{3}}{128 \pi f_{\pi}^{4}} \frac{\mathbf{q}}{q^{2}+m_{\pi}^{2}} \int_{0}^{1} d z\left[\tau _ { 2 , a } \left[-10 M\left(k_{1}, z\right)^{3}+M\left(k_{1}, z\right)\left(15 m_{\pi}^{2}+11 k_{1}^{2}\right.\right.\right. \\
& \left.\left.+3 k_{2}^{2}+3 q^{2}-20 k_{1}^{2} z \bar{z}\right)+\frac{k_{1}^{2} z \bar{z}}{M\left(k_{1}, z\right)}\left(5 m_{\pi}^{2}+k_{2}^{2}+q^{2}+3 k_{1}^{2}-2 k_{1}^{2} z \bar{z}\right)\right] \\
& \left.-2\left(\boldsymbol{\tau}_{1} \times \boldsymbol{\tau}_{2}\right)_{a}\left(\boldsymbol{\sigma}_{1} \times \mathbf{k}_{1}\right) \cdot\left(\mathbf{k}_{2}+\mathbf{q}\right) M\left(k_{1}, z\right)\right] \frac{\boldsymbol{\sigma}_{2} \cdot \mathbf{k}_{2}}{\omega_{2}^{2}}, \\
& \mathbf{j}_{5, a}^{(1)}(\mathrm{e} 17)=\frac{g_{A}^{3}}{32 \pi} \frac{m_{\pi}^{3}}{f_{\pi}^{4}} \tau_{2, a} \frac{\mathbf{q}}{q^{2}+m_{\pi}^{2}} \frac{\boldsymbol{\sigma}_{2} \cdot \mathbf{k}_{2}}{\omega_{2}^{2}},
\end{aligned}
$$


where $M(k, z)$ and $\bar{z}$ have been defined in Eqs. (199). The contributions corresponding to diagrams e2, e9, and e21 easily follow from those for e1, e8, and e20. The loop functions $W_{i}$ and $Z_{i}$ introduced in Eqs. (250) and (251) are defined as

$$
\begin{aligned}
W_{1}(k)= & \int_{0}^{1} d z\left[\left(1-5 g_{A}^{2}\right) M(k, z)-\frac{g_{A}^{2} k^{2}}{2}\left[\frac{9 z \bar{z}-1}{M(k, z)}+\frac{k^{2}(z \bar{z})^{2}}{4 M(k, z)^{3}}\right]\right], \\
W_{2}(k)= & \int_{0}^{1} d z\left[-\frac{g_{A}^{2}(z \bar{z})^{2} k^{2}}{M(k, z)^{3}}+\frac{z \bar{z}\left(7 g_{A}^{2}+2\right)-g_{A}^{2}}{2 M(k, z)}\right] \\
W_{3}(k)= & -\frac{1}{2} \int_{0}^{1} d z \frac{1}{M(k, z)}, \\
Z_{1}(k)= & \int_{0}^{1} d z\left[\frac{z \bar{z} k^{2}}{M(k, z)}+3 M(k, z)\right] \\
Z_{2}(\mathbf{k})= & \int_{0}^{1} d z\left[4 m_{\pi}^{3}-10 M(k, z)^{3}+M(k, z)\left(15 m_{\pi}^{2}+14 k^{2}-6 \mathbf{q} \cdot \mathbf{k}+6 q^{2}\right.\right. \\
& \left.\left.-20 z \bar{z} k^{2}\right)+\frac{z \bar{z} k^{2}}{M(k, z)}\left(5 m_{\pi}^{2}+4 k^{2}+2 q^{2}-2 \mathbf{q} \cdot \mathbf{k}-2 k^{2} z \bar{z}\right)\right], \\
Z_{3}(k)= & \int_{0}^{1} d z M(k, z) .
\end{aligned}
$$




\section{APPENDIX J}

\section{LEADING ORDER AXIAL CHARGE IN COVARIANT PERTURBATION THEORY}

We discuss here how the one-body axial charge operator emerges in covariant perturbation theory. The relevant interaction Hamiltonian densities are

$$
\begin{aligned}
\mathcal{H}_{\pi A}(x) & =f_{\pi} \mathbf{A}^{0}(x) \cdot \boldsymbol{\Pi}(x), \\
\mathcal{H}_{\pi N N}^{(\mathrm{a})}(x) & =\frac{g_{A}}{2 f_{\pi}} \bar{N}(x) \boldsymbol{\tau} \cdot \boldsymbol{\Pi}(x) \gamma^{0} \gamma^{5} N(x), \\
\mathcal{H}_{\pi N N}^{(\mathrm{b})}(x) & =\frac{g_{A}}{2 f_{\pi}} \bar{N}(x) \boldsymbol{\tau} \cdot \partial_{i} \boldsymbol{\pi}(x) \gamma^{i} \gamma^{5} N(x),
\end{aligned}
$$

where all fields are in interaction picture.

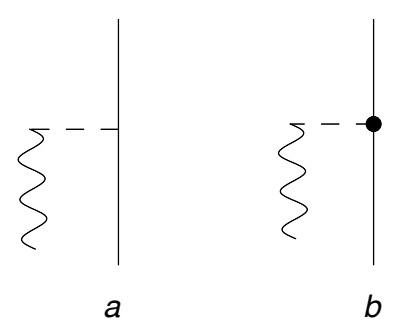

FIG. 37: Feynman amplitudes contributing to the one-body axial charge at leading order.

The $S$-matrix elements associated with the Feynman amplitudes in Fig. 37 are given by

$$
S_{f i}^{(\gamma)}=-\frac{1}{2} \int \mathrm{d}^{4} x \mathrm{~d}^{4} y\left\langle\mathbf{p}^{\prime}, \lambda^{\prime}\left|T\left[\mathcal{H}_{\pi A}(x) \mathcal{H}_{\pi N N}^{(\gamma)}(y)+\mathcal{H}_{\pi N N}^{(\gamma)}(x) \mathcal{H}_{\pi A}(y)\right]\right| \mathbf{p}, \lambda\right\rangle
$$

where $\gamma=\mathrm{a}$ or b, $T$ denotes the usual chronological product. Then for $\gamma=$ a we obtain

$$
\begin{aligned}
S_{f i}^{(\mathrm{a})}= & -\frac{g_{A}}{8 m} \chi_{\lambda^{\prime}}^{\dagger} \boldsymbol{\sigma} \cdot\left(\mathbf{p}^{\prime}+\mathbf{p}\right) A_{c}^{0} \tau_{d} \chi_{\lambda} \int \mathrm{d}^{4} x \mathrm{~d}^{4} y\left[\mathrm{e}^{i\left(p^{\prime}-p\right) \cdot y-i q \cdot x}\left\langle 0\left|T\left[\Pi_{c}(x) \Pi_{d}(y)\right]\right| 0\right\rangle\right. \\
& \left.+\mathrm{e}^{i\left(p^{\prime}-p\right) \cdot x-i q \cdot y}\left\langle 0\left|T\left[\Pi_{d}(x) \Pi_{c}(y)\right]\right| 0\right\rangle\right],
\end{aligned}
$$

where we have considered the leading order in the non-relativistic expansion of the nucleon matrix element. Since in the interaction picture the conjugate field momentum $\Pi_{c}(x)=$ 
$\partial^{0} \pi_{c}(x)$, it is easily seen that (see also Ref. [86])

$$
\begin{aligned}
\left\langle 0\left|T\left[\Pi_{c}(x) \Pi_{d}(y)\right]\right| 0\right\rangle & =\partial_{x}^{0} \partial_{y}^{0}\left\langle 0\left|T\left[\pi_{c}(x) \pi_{d}(y)\right]\right| 0\right\rangle-i \delta_{c d} \delta\left(x^{0}-y^{0}\right) \delta(\mathbf{x}-\mathbf{y}) \\
& =-i \delta_{c d} \int \frac{d^{4} k}{(2 \pi)^{4}} e^{-i k \cdot(x-y)}\left(1+\frac{k_{0}^{2}}{m_{\pi}^{2}-k^{2}-i \epsilon}\right)
\end{aligned}
$$

with the Feynman propagator defined by

$$
\left\langle 0\left|T\left[\pi_{c}(x) \pi_{d}(y)\right]\right| 0\right\rangle=\int \frac{d^{4} k}{(2 \pi)^{4}} \frac{-i \delta_{c d}}{m_{\pi}^{2}-k^{2}-i \epsilon} e^{-i k \cdot(x-y)} .
$$

The $T$-matrix element $T_{f i}$ obtained from $S_{f i}=-i(2 \pi)^{4} \delta\left(p^{\prime}-p-q\right) T_{f i}$ reads

$$
T_{f i}^{(\mathrm{a})}=-\frac{g_{A}}{4 m} A_{c}^{0} \chi_{\lambda^{\prime}}^{\dagger} \boldsymbol{\sigma} \cdot\left(\mathbf{p}^{\prime}+\mathbf{p}\right) \tau_{c} \chi_{\lambda}\left(1+\frac{q_{0}^{2}}{m_{\pi}^{2}+\mathbf{q}^{2}-q_{0}^{2}-i \epsilon}\right)
$$

where the term proportional to $q_{0}=p_{0}^{\prime}-p_{0}$ is suppressed by $Q^{2}$ in the power counting. The leading order term leads to the axial charge operator in Eq. (148). A similar analysis shows that the leading-order contribution to $S_{f i}^{(\mathrm{b})}$ vanishes.

As already noted, the interaction Hamiltonian in Eq. (75) contains no direct coupling of $A_{a}^{0}$ to the nucleon. However, diagrams of the type illustrated in Fig. 37 are not considered in Refs. $[28,48]$. It would appear that their contribution is accounted for by retaining the term $-i \delta_{c d} \delta(x-y)$ in Eq. (544), which effectively leads to a direct coupling between $A_{a}^{0}$ and the nucleon. 


\section{APPENDIX K}

\section{CONFIGURATION-SPACE EXPRESSIONS}

The Fourier transforms of two-body operators are easily reduced to one-dimensional integrals [or two-dimensional ones in the case of the N4LO(MPE) operator], which can be conveniently evaluated by Gaussian quadrature formulae. For example, the N3LO(OPE) current is given by

$$
\mathbf{j}_{ \pm}^{\mathrm{N} 3 \mathrm{LO}}(\mathrm{OPE})=\mathbf{j}_{ \pm}^{\mathrm{N} 3 \mathrm{LO}}\left(c_{3}\right)+\mathbf{j}_{ \pm}^{\mathrm{N} 3 \mathrm{LO}}\left(c_{4}\right)+\mathbf{j}_{ \pm}^{\mathrm{N} 3 \mathrm{LO}}(\mathrm{nl})
$$

where

$$
\begin{aligned}
& \mathbf{j}_{ \pm}^{\mathrm{N} 3 \mathrm{LO}}\left(c_{3}\right)=-\tau_{2, \pm}\left[\frac{F_{1}\left(z ; c_{3}\right)}{z} \boldsymbol{\sigma}_{2}+F_{2}\left(z ; c_{3}\right) \hat{\mathbf{z}}\left(\boldsymbol{\sigma}_{2} \cdot \hat{\mathbf{z}}\right)\right]+(1 \rightleftharpoons 2) \\
& \mathbf{j}_{ \pm}^{\mathrm{N} 3 \mathrm{LO}}\left(c_{4}\right)=-\left(\boldsymbol{\tau}_{1} \times \boldsymbol{\tau}_{2}\right)_{ \pm} \boldsymbol{\sigma}_{1} \times\left[\frac{F_{1}\left(z ; c_{4}\right)}{z} \boldsymbol{\sigma}_{2}+F_{2}\left(z ; c_{4}\right) \hat{\mathbf{z}}\left(\boldsymbol{\sigma}_{2} \cdot \hat{\mathbf{z}}\right)\right]+(1 \rightleftharpoons 2) \\
& \mathbf{j}_{ \pm}^{\mathrm{N} 3 \mathrm{LO}}(\mathrm{nl})=-\left(\boldsymbol{\tau}_{1} \times \boldsymbol{\tau}_{2}\right)_{ \pm}\left\{-i \boldsymbol{\nabla}_{1}^{z}, F_{1}(z ; \mathrm{nl}) \boldsymbol{\sigma}_{2} \cdot \hat{\mathbf{z}}\right\}+(1 \rightleftharpoons 2)
\end{aligned}
$$

Here we have defined $\mathbf{r}=\mathbf{r}_{1}-\mathbf{r}_{2}$, the adimensional variable $\mathbf{z}=\Lambda \mathbf{r},-i \boldsymbol{\nabla}_{i}^{z}$ as the adimensional momentum operator, and the radial functions

$$
\begin{aligned}
& F_{1}\left(z ; c_{3}\right)=-\frac{1}{\pi^{2}} \frac{g_{A} \bar{c}_{3}}{\bar{f}_{\pi}^{2}} \int_{0}^{\infty} d x \frac{x^{3}}{x^{2}+\bar{m}_{\pi}^{2}} \mathrm{e}^{-x^{4}} j_{1}(x z), \\
& F_{2}\left(z ; c_{3}\right)=\frac{1}{\pi^{2}} \frac{g_{A} \bar{c}_{3}}{\bar{f}_{\pi}^{2}} \int_{0}^{\infty} d x \frac{x^{4}}{x^{2}+\bar{m}_{\pi}^{2}} \mathrm{e}^{-x^{4}} j_{2}(x z)
\end{aligned}
$$

where $j_{n}(x z)$ are spherical Bessel functions. We have also introduced adimensional constants (denoted with the overline) expressing them units of the cutoff $\Lambda$. They are given by

$$
\bar{m}_{\pi}=m_{\pi} / \Lambda, \quad \bar{m}=m / \Lambda, \quad \bar{f}_{\pi}=f_{\pi} / \Lambda, \quad \bar{c}_{3}=c_{3} \Lambda, \quad \bar{c}_{4}=c_{4} \Lambda
$$

The functions $F_{1}\left(z ; c_{4}\right)$ and $F_{2}\left(z ; c_{4}\right)$, and $F_{1}(z ; \mathrm{nl})$ follow from those above by the replacement of the pre-factor as

$$
\begin{aligned}
& \frac{1}{\pi^{2}} \frac{g_{A} \bar{c}_{3}}{\bar{f}_{\pi}^{2}} \longrightarrow \frac{1}{4 \pi^{2}} \frac{g_{A}}{\bar{f}_{\pi}^{2}}\left(\bar{c}_{4}+\frac{1}{4 \bar{m}}\right) \text { for } F_{1}\left(z ; c_{4}\right) \text { and } F_{2}\left(z ; c_{4}\right) \\
& \frac{1}{\pi^{2}} \frac{g_{A} \bar{c}_{3}}{\bar{f}_{\pi}^{2}} \longrightarrow \frac{1}{16 \pi^{2}} \frac{g_{A}}{\bar{m} \bar{f}_{\pi}^{2}} \text { for } F_{1}(z ; \mathrm{nl}) .
\end{aligned}
$$


The Fourier transform of the three-body operator is given by

$$
\begin{aligned}
\mathbf{j}_{ \pm}^{\mathrm{N} 4 \mathrm{LO}}(3 \mathrm{~B})= & \sum_{\text {cyc }}\left(2 \tau_{1, \pm} \boldsymbol{\tau}_{2} \cdot \boldsymbol{\tau}_{3}-\tau_{2, \pm} \boldsymbol{\tau}_{3} \cdot \boldsymbol{\tau}_{1}-\tau_{3, \pm} \boldsymbol{\tau}_{1} \cdot \boldsymbol{\tau}_{2}\right) \\
& \times \boldsymbol{\sigma}_{1}\left(\boldsymbol{\sigma}_{2} \cdot \hat{\mathbf{z}}_{12}\right)\left(\boldsymbol{\sigma}_{3} \cdot \hat{\mathbf{z}}_{13}\right) F_{1}\left(z_{12} ; 3 \mathrm{~B}\right) F_{1}\left(z_{13} ; 3 \mathrm{~B}\right)
\end{aligned}
$$

and the function $F_{1}(z ; 3 \mathrm{~B})$ is obtained from $F_{1}\left(z ; c_{3}\right)$ by replacing

$$
\frac{1}{\pi^{2}} \frac{g_{A} \bar{c}_{3}}{\bar{f}_{\pi}^{2}} \longrightarrow \frac{1}{4 \sqrt{2} \pi^{2}} \frac{g_{A}^{3 / 2}}{\bar{f}_{\pi}^{2}}
$$

The Fourier transform of the second term in the N4LO(3B) current of Eq. (294) proportional to $\boldsymbol{\sigma}_{1} \cdot \mathbf{k}_{1}$ can be reduced to a two-dimensional parametric integral. To this end we first regularize it as,

$$
\begin{aligned}
& \text { 2nd term }=\sum_{\text {cyc }} \frac{g_{A}^{3}}{6 f_{\pi}^{4}} \text { (isospin) } \boldsymbol{\sigma}_{3} \cdot \nabla_{3} \boldsymbol{\sigma}_{2} \cdot \nabla_{2} \boldsymbol{\sigma}_{1} \cdot \nabla_{1} \nabla_{1} I, \\
& I=\int \frac{d \mathbf{k}_{2}}{(2 \pi)^{3}} \frac{d \mathbf{k}_{3}}{(2 \pi)^{3}} C_{\Lambda}\left(\left|\mathbf{k}_{2}+\mathbf{k}_{3}\right|\right) \mathrm{e}^{-i\left(\mathbf{k}_{2} \cdot \mathbf{r}_{12}+\mathbf{k}_{3} \cdot \mathbf{r}_{13}\right)} \frac{1}{\omega_{\mathbf{k}_{2}+\mathbf{k}_{3}}^{2} \omega_{\mathbf{k}_{2}}^{2} \omega_{\mathbf{k}_{3}}^{2}},
\end{aligned}
$$

where (isospin) stands for the isospin factor in parentheses of Eq. (556). After changing variables to $\mathbf{k}_{2}=\mathbf{P} / 2+\mathbf{p}$ and $\mathbf{k}_{3}=\mathbf{P} / 2-\mathbf{p}$, making use of Feynman's parametrization for the denominator $1 /\left(\omega_{\mathbf{P} / 2+\mathbf{k}} \omega_{\mathbf{P} / 2-\mathbf{k}}\right)$, and carrying out the angular integration over the $\mathbf{P}$ directions, we find

$$
I=\frac{1}{16 \pi^{3}} \int_{-1 / 2}^{1 / 2} d y \int_{0}^{\infty} d P P^{2} \frac{\mathrm{e}^{-(P / \Lambda)^{4}}}{P^{2}+m_{\pi}^{2}} j_{0}\left(P\left|\mathbf{r}_{1}-\mathbf{R}_{23}+y \mathbf{r}_{23}\right|\right) \mathrm{e}^{-L(P, y) r_{23}} \frac{1}{L(P, y)},
$$

where

$$
L(P, y)=\sqrt{m_{\pi}^{2}+P^{2}\left(1 / 4-y^{2}\right)} .
$$

In terms of adimensional variables, the current now reads

$$
\begin{aligned}
\text { 2nd term }= & \sum_{\operatorname{cyc}} \frac{g_{A}^{3}}{96 \pi^{3} \bar{f}_{\pi}^{4}} \text { (isospin) } \boldsymbol{\sigma}_{3} \cdot \boldsymbol{\nabla}_{3}^{z} \boldsymbol{\sigma}_{2} \cdot \boldsymbol{\nabla}_{2}^{z} \boldsymbol{\sigma}_{1} \cdot \boldsymbol{\nabla}_{1}^{z} \boldsymbol{\nabla}_{1}^{z} \int_{-1 / 2}^{1 / 2} d y \int_{0}^{\infty} d x x^{2} \frac{\mathrm{e}^{-x^{4}}}{x^{2}+\bar{m}_{\pi}^{2}} \\
& \times \frac{\mathrm{e}^{-\bar{L}(x, y) z}}{\bar{L}(x, y)} j_{0}(x|\mathbf{Z}+y \mathbf{z}|),
\end{aligned}
$$

where the gradients are relative to $\mathbf{z}_{i}=\Lambda \mathbf{r}_{i}$, and we have defined $\mathbf{Z}=\Lambda\left(\mathbf{r}_{1}-\mathbf{R}_{23}\right)$ and $\mathbf{z}=\Lambda \mathbf{r}_{23}$, and

$$
\bar{L}(x, y)=\sqrt{\bar{m}_{\pi}^{2}+x^{2}\left(1 / 4-y^{2}\right)} .
$$


In order to evaluate the gradients, we introduce the Jacobi variables,

$$
\nabla_{1}^{z}=\nabla^{Z}, \quad \nabla_{2}^{z}=-\frac{1}{2} \nabla^{Z}+\nabla^{z}, \quad \nabla_{3}^{z}=-\frac{1}{2} \nabla^{Z}-\nabla^{z}
$$

where the gradients $\boldsymbol{\nabla}^{Z}$ and $\boldsymbol{\nabla}^{z}$ are now relative to $\mathbf{Z}$ and $\mathbf{z}$, respectively. We obtain

$$
\begin{aligned}
& \sigma_{3, \delta} \sigma_{2, \gamma} \sigma_{1, \beta}\left(\frac{1}{4} \nabla_{\delta}^{Z} \nabla_{\gamma}^{Z}-\nabla_{\delta}^{z} \nabla_{\gamma}^{z}-\frac{1}{2} \nabla_{\delta}^{Z} \nabla_{\gamma}^{z}+\frac{1}{2} \nabla_{\delta}^{z} \nabla_{\gamma}^{Z}\right)\left[\mathrm{e}^{-\bar{L} z} \nabla_{\beta}^{Z} \nabla_{\alpha}^{Z} j_{0}(x|\mathbf{Z}+y \mathbf{z}|)\right] \\
= & x^{2} \mathrm{e}^{-\bar{L} z} \sigma_{3, \delta} \sigma_{2, \gamma} \sigma_{1, \beta}\left\{x^{2}\left(\frac{1}{4}-y^{2}\right) \nabla_{\delta}^{t} \nabla_{\gamma}^{t} \nabla_{\beta}^{t} \nabla_{\alpha}^{t}-x \bar{L}\left(\frac{1}{2}-y\right) \hat{z}_{\delta} \nabla_{\gamma}^{t} \nabla_{\beta}^{t} \nabla_{\alpha}^{t}\right. \\
& \left.+x \bar{L}\left(\frac{1}{2}+y\right) \hat{z}_{\gamma} \nabla_{\delta}^{t} \nabla_{\beta}^{t} \nabla_{\alpha}^{t}-\left[\bar{L}^{2}\left(1+\frac{1}{\bar{L} z}\right) \hat{z}_{\delta} \hat{z}_{\gamma}-\frac{\bar{L}}{z} \delta_{\gamma \delta}\right] \nabla_{\beta}^{t} \nabla_{\alpha}^{t}\right\} j_{0}(t),
\end{aligned}
$$

where we have defined $\mathbf{t}=x \mathbf{Z}+x y \mathbf{z}$ and the corresponding gradient $\boldsymbol{\nabla}^{t}$. By making use of the identities

$$
\begin{aligned}
\nabla_{\beta}^{t} \nabla_{\alpha}^{t} j_{0}(t)= & \delta_{\alpha \beta}\left(\frac{1}{t} \frac{d}{d t}\right) j_{0}(t)+t_{\alpha} t_{\beta}\left(\frac{1}{t} \frac{d}{d t}\right)^{2} j_{0}(t) \\
\nabla_{\gamma}^{t} \nabla_{\beta}^{t} \nabla_{\alpha}^{t} j_{0}(t)= & \left(\delta_{\alpha \beta} t_{\gamma}+\delta_{\alpha \gamma} t_{\beta}+\delta_{\beta \gamma} t_{\alpha}\right)\left(\frac{1}{t} \frac{d}{d t}\right)^{2} j_{0}(t)+t_{\alpha} t_{\beta} t_{\gamma}\left(\frac{1}{t} \frac{d}{d t}\right)^{3} j_{0}(t),(56 t \\
\nabla_{\delta}^{t} \nabla_{\gamma}^{t} \nabla_{\beta}^{t} \nabla_{\alpha}^{t} j_{0}(t)= & \left(\delta_{\alpha \beta} \delta_{\gamma \delta}+\delta_{\alpha \gamma} \delta_{\beta \delta}+\delta_{\beta \gamma} \delta_{\alpha \delta}\right)\left(\frac{1}{t} \frac{d}{d t}\right)^{2} j_{0}(t)+\left(\delta_{\alpha \beta} t_{\gamma} t_{\delta}+\delta_{\alpha \gamma} t_{\beta} t_{\delta}\right. \\
& \left.+\delta_{\beta \gamma} t_{\alpha} t_{\delta}+\delta_{\alpha \delta} t_{\beta} t_{\gamma}+\delta_{\beta \delta} t_{\alpha} t_{\gamma}+\delta_{\gamma \delta} t_{\alpha} t_{\beta}\right)\left(\frac{1}{t} \frac{d}{d t}\right)^{3} j_{0}(t) \\
& +t_{\alpha} t_{\beta} t_{\gamma} t_{\delta}\left(\frac{1}{t} \frac{d}{d t}\right)^{4} j_{0}(t)
\end{aligned}
$$

and

$$
\left(\frac{1}{t} \frac{d}{d t}\right)^{m} j_{0}(t)=(-)^{m} \frac{1}{t^{m}} j_{m}(t),
$$

the current in Eq. (562) is reduced to a sum of terms depending on parametric integrals in $x$ and $y$. While the matrix element of 2 nd term could in principle be evaluated, the computational effort required to do so in the present Monte Carlo calculations is, however, too large (and unjustified in view of its expected contribution, see Table 1). For this reason it has been neglected in Ch. 6 of the present Thesis. 


\title{
VITA
}

\begin{abstract}
Alessandro Baroni
Department of Physics

Old Dominion University

Norfolk, VA 23529
\end{abstract}

Università di Pisa, Pisa, Italy

- B.S. Physics, 110/110, January 2007

Università di Pisa, Pisa, Italy

- M.Sc. Theoretical Physics, Summa cum Laude, February 2011

Thesis Title: Parity Violation in two nucleon systems

Advisor: Prof. Michele Viviani

Old Dominion University, Norfolk, VA

- Ph.D. Physics, August 2017 (expected)

Thesis Title: Chiral effective field theory analysis of weak axial currents in few-nucleon systems

Advisor: Prof. Rocco Schiavilla

\section{Professional Experience}

- Research Assistant August 2011 - present

Department of Physics Old Dominion University, Norfolk, VA and Theory Center, Thomas Jefferson National Accelerator Facility, Newport News, VA

- Teaching Assistant Spring 2014, Spring 2015

Department of Physics Old Dominion University, Norfolk, VA

\section{Awards}

- JSA/Jefferson Laboratory Distinguished Theory Studentship, Fall 2011-Fall 2013

- Physics Qualifying Examination award, Fall 2012

Typeset using $\mathrm{ATEX}_{\mathrm{E}}$ 